\title{
Resting-state fMRI neurodynamics in neuropsychiatric disorders
}

Citation for published version (APA):

Bernas, A. (2020). Resting-state fMRI neurodynamics in neuropsychiatric disorders. [Doctoral Thesis, Maastricht University]. Gildeprint Drukkerijen. https://doi.org/10.26481/dis.20200929abe

Document status and date:

Published: 01/01/2020

DOI:

10.26481/dis.20200929abe

Document Version:

Publisher's PDF, also known as Version of record

\section{Please check the document version of this publication:}

- A submitted manuscript is the version of the article upon submission and before peer-review. There can be important differences between the submitted version and the official published version of record.

People interested in the research are advised to contact the author for the final version of the publication, or visit the DOI to the publisher's website.

- The final author version and the galley proof are versions of the publication after peer review.

- The final published version features the final layout of the paper including the volume, issue and page numbers.

Link to publication

\footnotetext{
General rights rights.

- You may freely distribute the URL identifying the publication in the public portal. please follow below link for the End User Agreement:

www.umlib.nl/taverne-license

Take down policy

If you believe that this document breaches copyright please contact us at:

repository@maastrichtuniversity.nl

providing details and we will investigate your claim.
}

Copyright and moral rights for the publications made accessible in the public portal are retained by the authors and/or other copyright owners and it is a condition of accessing publications that users recognise and abide by the legal requirements associated with these

- Users may download and print one copy of any publication from the public portal for the purpose of private study or research.

- You may not further distribute the material or use it for any profit-making activity or commercial gain

If the publication is distributed under the terms of Article $25 \mathrm{fa}$ of the Dutch Copyright Act, indicated by the "Taverne" license above, 
MAASTRICHT UNIVERSITY

DOCTORAL THESIS

\section{Resting-state fMRI neurodynamics in neuropsychiatric disorders}

Author:

Antoine BERNAS
Promotor: Prof. dr. Albert P. AldenKAMP

Co-promotor: Dr. Sveta ZINGER

A thesis submitted in fulfillment of the requirements

for the degree of Doctor of Philosophy

in the

School for Mental Health and Neuroscience

Clinical Neurosciences

July, 2020 
Copyright (c) 2020 by Antoine Bernas - All rights reserved.

No part of this publication may be reproduced, stored in a retrieval system or transmitted in any form or by means, electronic, mechanical, photocopy, recording or otherwise, without the prior permission of the author.

Printed by Gildeprint, the Netherlands

Layout by Antoine Bernas

Cover design by Antoine Bernas

Cover background image from https://unsplash.com/@andyjh07

ISBN: 978-94-6419-006-9

The printing of this thesis was financially supported by Maastricht University 


\title{
Resting-state fMRI neurodynamics in neuropsychiatric disorders
}

\author{
DISSERTATION
}

to obtain the degree of Doctor at the Maastricht University, on the authority of the Rector Magnificus, Prof. dr. Rianne M. Letschert in accordance with the decision of the Board of Deans, to be defended in public on Tuesday, September 29, 2020, at 10:00 am.

by 


\section{Supervisor}

Prof. dr. A.P. Aldenkamp

\section{Co-supervisor}

Dr. ir. S. Zinger (Eindhoven University of Technology)

\section{Assessment Committee}

Prof. dr. D.E.J Linden (Chairman)

Prof. dr. R.J van Oostenbrugge

Prof. dr. R. van Ee (Donders Institute Nijmegen \& Philips Research)

Prof. dr. ir. M. Breeuwer (Eindhoven University of Technology \& Philips Healthcare) 


\section{Summary}

Neuropsychiatric disorders such as schizophrenia, autism, mild cognitive impairment and depression are described as neurological conditions with psychiatric symptoms. An important issue in these diseases is the lack of brain biomarkers, which causes diagnostic uncertainty and lack of treatment navigation. Finding these neurobiomarkers by means of neuroimaging techniques, such as functional MRI, is the core of psychoradiology—a relatively new field of research.

In this thesis we attempt to contribute to this research field by investigating new ways of assessing brain dynamics using resting-state functional MRI (rs-fMRI). More specifically, three methods to assess neurodynamics are evaluated: the Granger causality (measure of effective connectivity), the wavelet coherence (spectro-temporal analysis), and the evolutionary game theory approach on networks. Neuropsychiatry is a vast field of research, thus, in order to narrow the focus, these novel approaches to brain dynamics assessment are tested on two neuropsychiatric disorders: the accelerated cognitive ageing (ACA) observed in elderly with epilepsy, and the autism spectrum disorder (ASD).

The main goals of this research on discovering new biomarkers for neuropsychiatric disorders and the utility of brain dynamics in rs-fMRI are introduced in Chapter 1. This introductory chapter also contains a review of the state-of-the-art of the current solutions for finding these fMRI-based neurobiomarkers and the description of the three novel abovementioned dynamics methods.

In Chapter 2, static functional connectivity (sFC) between large-scale resting-state networks (RSNs) is measured in ACA and compared to age- and education-level-matched healthy elderly in two resting-state fMRI sessions (pre- and post-task). Within the lateralised executive networks, i.e. fronto-parietal networks, the static connectivity was slightly weakened in both resting states. Regarding the between-network sFC analysis, the default mode network (DMN) had a weaker and anti-correlated connectivity with the ventral attention network (VAN) in ACA patients as compared to the control group in the pre-task resting state. At post-task, i.e. after a cognitively demanding task, the strength of the connectivity between the salience network (SN) and the dorsal attention network (DAN) was also decreased, whereas the DMN connectivity with the VAN increased and was not significantly different from the DMN-VAN connectivity in controls anymore. In general, the network functional connectivity amount networks tended to change more in ACA than in healthy controls, where the emphasis was shifted to connectivity between the DMN and the cognitive-control networks (FPR, VAN, DAN and SN). In this chapter we conclude that the switching of activity between the DMN, SN and executive network appears less efficient or delayed in ACA patients, which might be an echo of a loss of segregation. This loss of segregation also appears naturally in 'healthy' ageing, which corroborates the idea of a faster cognitive decline in our patients with ACA.

After investigating the sFC between large-scale cognitive networks, we explore the effective connectivity, i.e. the directed causal influences between the networks in terms of Granger causality in Chapter 3. This is performed on the same ACA patients and healthy controls. In addition, the correlation between the causality values and the deterioration in IQ scores is assessed. As seen with static connectivity, the GC from executive control and attention networks are also challenged in ACA patients. More specifically the directed connectivity from the cingulate cortex towards 
the DAN, fronto-parietal right and the $\mathrm{SN}$ is stronger in the patients as compared to the control group in the pre-task rs-fMRI. After the task, this tendency of over-recruitment is reduced and statistically non-significant. When we assessed the association between GC and the cognitive decline measurements, our main findings reveal that in both rs-fMRI scans (pre- and post-task) the stronger GC from the DMN to the VAN is correlated with a stronger decline in perceptual reasoning index (most deficient IQ scores in ACA patients, related to fluid intelligence). These results are corroborated by the $\mathrm{CRUNCH}^{1}$ and PASA ${ }^{2}$ models of brain ageing. Hence, these findings are in line with our accelerated cognitive ageing hypothesis.

In Chapter 4, the effective connectivity between large-scale cognitive and limbic (emotion-related) RSNs is measured in ASD and compared to age- and IQ-matched healthy adolescents in two resting-state fMRI sessions. First, the within-network sFC, i.e. the spatial distribution and strength of activation of the RSNs are not different between patients with ASD and controls. However, the between-network causality shows discrepancies. The adolescents display also different results in GC when comparing the two resting-states sessions. Indeed, our results suggest that the dynamic causal measurement from the ventral attention network towards the executive-salience network is weakened in ASD patients, after an emotional task is performed, i.e. after triggering, or challenging, the brain cognitive state of the patients prior to the resting-state evaluation. These networks are known to be involved in emotion processing as well as involuntary cognitive processing, which are usually impaired for patients with autism.

A novel dynamics metric derived from wavelet-coherence maps is investigated in the adolescents with autism in Chapter 5 . In this chapter we aim at designing methodology for objective diagnosis of autism. This metric is coined the time of in-phase coherence, which provides an indication of how long two RSNs are synchronized with each other. Classifiers for autism diagnosis are then trained with these times of in-phase coherence features. Leave-one-out cross validation is applied for performance evaluation. To assess inter-site robustness, we also trained our classifiers on the in-house data, and tested them on an independent rs-fMRI dataset from Leuven University. We distinguished ASD from non-ASD adolescents in both independent datasets at more than $86 \%$ accuracy. In the second experiment, classifying the Leuven dataset with classifiers trained with our in-house data, resulted in $80 \%$ accuracy (100\% sensitivity, $66.7 \%$ specificity). This study shows that synchronicities between the VAN and the two fronto-parietal networks are biomarkers of ASD, and wavelet coherence-based classifiers lead to robust results and could be used as an objective diagnostic tool for ASD.

Chapter 6 implements the evolutionary game theory approach on networks (EGN) onto activity time series of the ACA patients and the (age- and education-matched) controls. Here, we also tested classifiers with features based on a novel dynamics metric derived from the EGN model: the emulative powers of the networks. We compared these EGN-based classifiers with classifiers trained on GC, time of coherence, and static functional connectivity (sFC) features. Results show that features extracted from the EGN approach-the emulative powers-are the best descriptors of the ACA condition, using dynamics associated with the DMN and the DAN. With these dynamics markers, linear discriminant analysis could identify ACA at $82.9 \%$ accuracy. Using waveletcoherence (WCoh) features with decision-tree algorithm, and sFC features with support vector machine (SVM), ACA could be identified at $77.1 \%$ and $77.9 \%$ accuracy respectively. GC fell short of being a relevant biomarker with best classifiers having an average accuracy of $67.9 \%$. Combining EGN-, WCoh-, GC-, and sFC-based features increased the classification performance up to $95.8 \%$ accuracy using SVMThe dynamics of the networks that lead to the best classifier performances are known to be challenged in elderly, and the results are in line with the idea of ACA patients having an accelerated cognitive decline. These findings allow us to localise the functional

\footnotetext{
${ }^{1}$ Compensation-Related Utilization of Neural Circuits Hypothesis
}

${ }^{2}$ Posterior-Anterior Shift in Aging 
networks affected by ACA, which in turns could lead to targeted treatment. This classification pipeline is also promising and could help diagnosing other neuropsychiatric disorders, and contribute to the field of psychoradiology.

Because of the weak discriminative power of GC, as shown in the previous paragraph, Chapter 7 investigates an alternative method for measuring effective connectivity between large-scale RSNs: the Bayesian search for effective connectivity topology. The Bayesian search is first compared to Granger causality with simulated times series. After showing the benefit of the new method in theory, we applied the Bayesian search algorithm on RSN time series of healthy young adults; and we were able to show the effect of intensive music listening sessions on the dynamic connectivity of cognitive networks.

Finally, Chapter 8 draws conclusions about neurodynamics in neuropsychiatric disorders, based on the results obtained in this research, and discusses the limitations but also the contributions and application prospects of these new tools in psychoradiology. Future work for this field of research is also discussed. For instance, the use of multi-modal approaches to improve classification performances is mentioned; or refining our dynamics models at different levels of abstraction (in the top-down and bottom-up brain processes) and tailored to the specificities of neuropsychiatric disorders could also be an avenue to explore. These new explorations could further help developing robust biomarkers of mental disorders, which could improve not only automated diagnostic tools, but also provide advances in prognosis-in terms of disease progression or treatment responses. 



\section{Contents}

Summary $\quad$ i

1 Introduction $\quad 1$

1.1 Neuropsychiatric disorders and cognition . . . . . . . . . . . . . . . 3

1.1.1 General context of mental diseases . . . . . . . . . . . . . . . . . 3

1.1.2 Cognitive changes in neuropsychiatric diseases . . . . . . . . . . . 4

1.1.3 Helping diagnosis and treatment . . . . . . . . . . . . . . 5

1.2 The brain dynamics in fMRI . . . . . . . . . . . . . . . . . 6

1.2.1 Why functional MRI? . . . . . . . . . . . . . . . . 6

1.2.2 Structural and functional connectivity as neurobiomarkers . . . . . . . . . 7

1.2.3 Resting-state-fMRI and the benefit of spontaneous dynamics . . . . . . . . . 8

1.2.4 Classical models (static FC) . . . . . . . . . . . . . . . . . 9

1.2 .5 Dynamics models (dynamic FC) . . . . . . . . . . . . . . . . . . 11

1.3 Overview of the proposed approaches . . . . . . . . . . . . . . . . 14

1.3.1 Rs-fMRI preprocessing and functional parcellation of the brain . . . . . . . 14

1.3.2 Causality between brain networks . . . . . . . . . . . . . 16

1.3.3 Phase coherence of brain network activity . . . . . . . . . . . . . . 17

1.3.4 Game theory as descriptor of network activity . . . . . . . . . . . . . . 19

1.3 .5 Thesis outline . . . . . . . . . . . . . . . . 20

2 Functional connectivity in Accelerated Cognitive Ageing: an fMRI-evaluation of brain networks in cognitively deteriorated patients with epilepsy 23

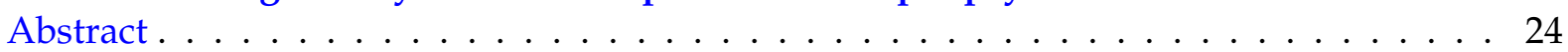

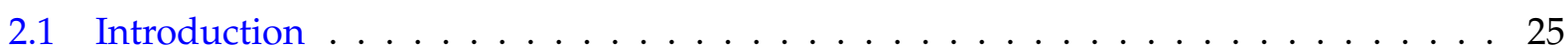

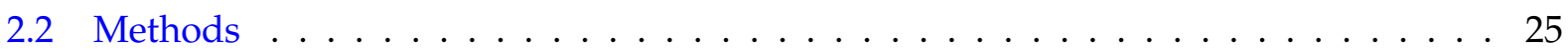

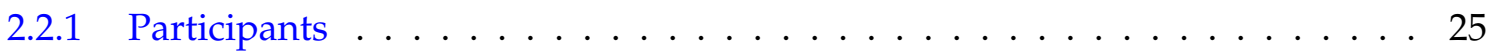

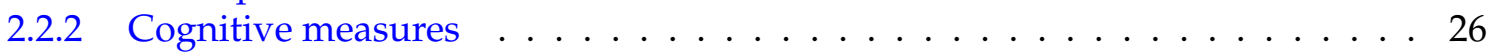

2.2 .3 MRI protocol . . . . . . . . . . . . . . . . 26

2.2.4 Multi-echo fMRI data pre-processing and resting-state network extraction . . 28

2.2 .5 RSN spatial analysis . . . . . . . . . . . . . . . . . . . 28

2.2 .6 RSN correlation and hierarchy . . . . . . . . . . . . . . . . . . . . . 29

2.2.7 RSN functional connectivity and cognitive measures . . . . . . . . . . . . . . 29

2.3 Results . . . . . . . . . . . . . . . . . . . . . . . . 29

2.3.1 Resting-state network extraction . . . . . . . . . . . . . . . . . . . . . 29

2.3.2 RSN spatial connectivity analysis . . . . . . . . . . . . . . . . . . . . . . . . 29

2.3 .3 RSN correlation and hierarchy . . . . . . . . . . . . . . . . . . 30

2.3.4 RSN functional connectivity and cognitive measures . . . . . . . . . . . 30

2.4 Discussion . . . . . . . . . . . . . . . . . . . . . . 34

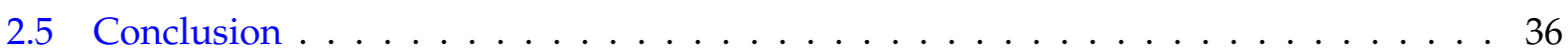

3 Accelerated Cognitive Ageing in epilepsy: exploring the effective connectivity between resting-state networks and its relation to cognitive decline 


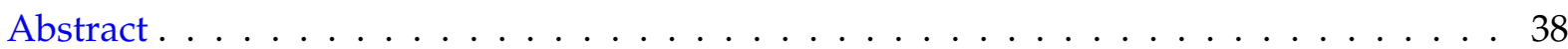

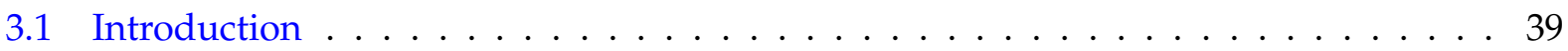

3.2 Methods . . . . . . . . . . . . . . . . . . . . . . . 39

3.2.1 Participants and cognitive measures . . . . . . . . . . . . . . . 39

3.2 .2 MRI protocol . . . . . . . . . . . . . . . . . . . . 40

3.2.3 Preprocessing and resting-state network extraction . . . . . . . . . . . . . . 40

3.2.4 Granger causality and statistical analyses . . . . . . . . . . . . . . . . . 42

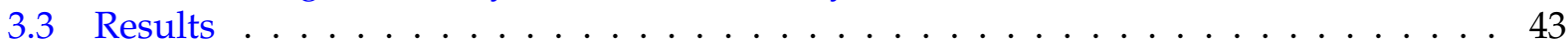

3.3.1 Resting-state networks selection . . . . . . . . . . . . . . 43

3.3.2 Granger causality differences . . . . . . . . . . . . . . . . . . . 43

3.3.3 Correlation between Granger causality and deterioration scores in ACA . . . 44

3.4 Discussion . . . . . . . . . . . . . . . . . . . . 46

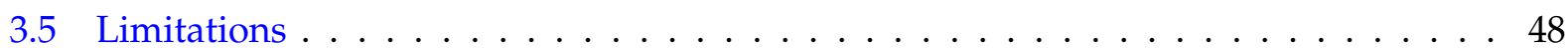

3.6 Conclusion . . . . . . . . . . . . . . . . . . . . . . . . . . . 49

4 Brain resting-state networks in adolescents with high-functioning autism: Analysis of spatial connectivity and temporal neurodynamics 51

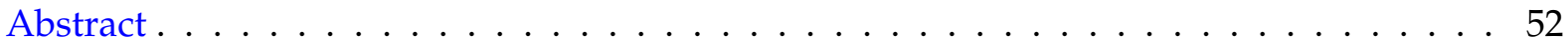

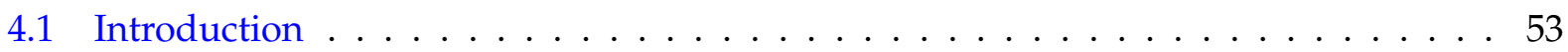

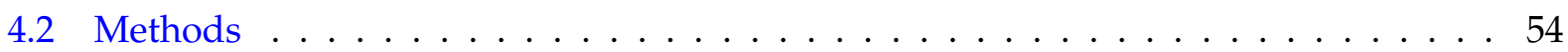

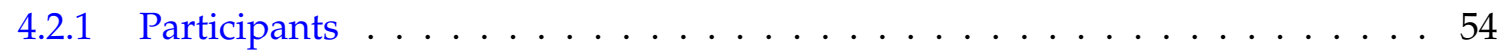

4.2 .2 Image acquisitions . . . . . . . . . . . . . . . 54

4.2 .3 Data preprocessing . . . . . . . . . . . . . . . . 55

4.2.4 Group independent component analysis . . . . . . . . . . . . . . 55

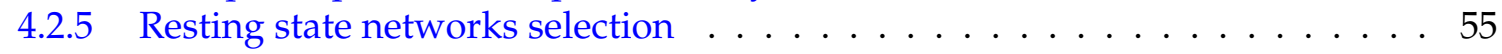

4.2 .6 Spatial RSN analysis between groups . . . . . . . . . . . . . . . . . 56

4.2 .7 Temporal dynamics of RSNs . . . . . . . . . . . . . . . . . 56

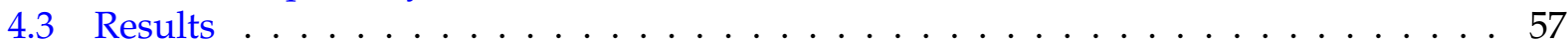

4.3.1 RSN selection and between-groups spatial analysis . . . . . . . . . . . . . 57

4.3 .2 Temporal dynamics of the RSNs . . . . . . . . . . . . . . . . . . 60

4.4 Discussion . . . . . . . . . . . . . . . . . . . . 60

4.4 Limitations . . . . . . . . . . . . . . . . . . . . 62

4.4.2 Methodological recommendations . . . . . . . . . . . . . 62

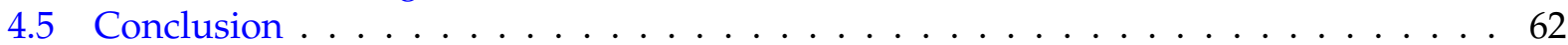

5 Wavelet coherence-based classifier: a resting-state functional MRI study on neurody$\begin{array}{ll}\text { namics in adolescents with high-functioning austism } & 63\end{array}$

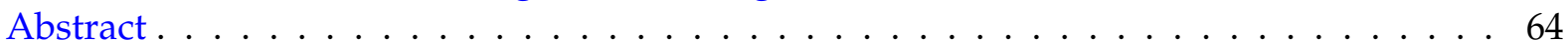

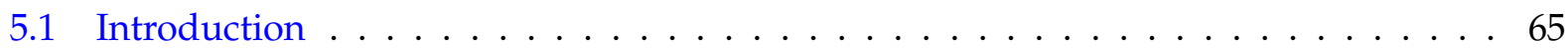

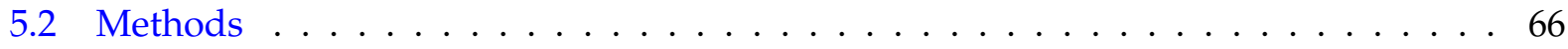

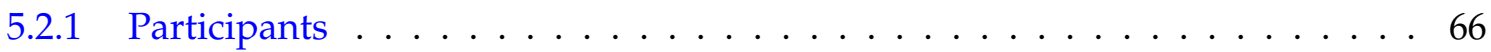

5.2 .2 Data acquisition . . . . . . . . . . . . . . . . . 67

5.2 .3 Resting-state ICA time-series extraction . . . . . . . . . . . . . . . . . . 67

5.2.4 Wavelet coherence and time of in-phase coherence . . . . . . . . . . . . 68

5.2 .5 Classification . . . . . . . . . . . . . . . . . . . . . 69

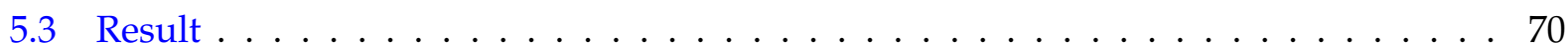

5.3.1 Resting-state network extraction . . . . . . . . . . . . . . . . 70

5.3.2 Wavelet coherence maps and time of in-phase coherence . . . . . . . . 71

5.3 .3 Time of in-phase coherence . . . . . . . . . . . . . . . . 71

5.3 .4 Classification and performance . . . . . . . . . . . . . . 72

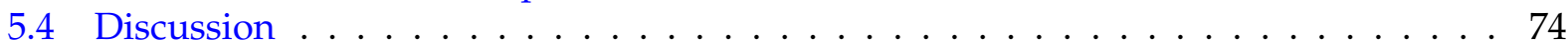




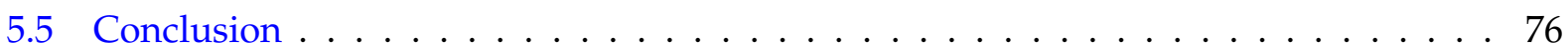

6 Emulative, coherent, and causal dynamics between large-scale brain networks are neurobiomarkers of accelerated cognitive ageing in epilepsy 77

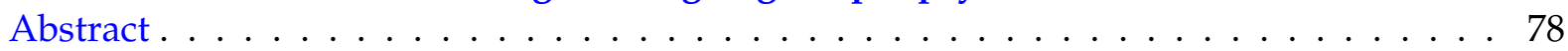

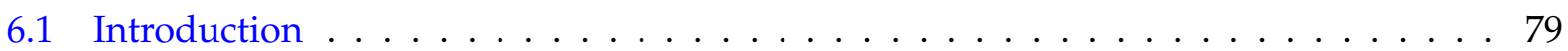

6.2 Materials and Methods . . . . . . . . . . . . . . . . . . . . . . 81

6.2.1 Dataset, preprocessing, and brain network extraction . . . . . . . . . 83

6.2.2 Static connectivity, causality, phase-coherence, and emulative power . . . . . 85

6.2.3 Selection of the most relevant features and classification analysis . . . . . . . 86

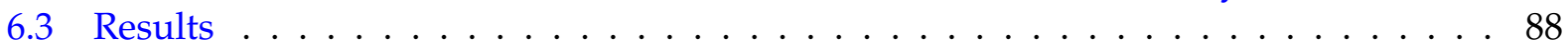

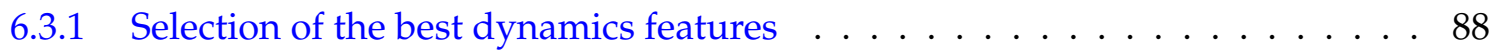

6.3 .2 Classification performances . . . . . . . . . . . . . . . 89

6.4 Discussion . . . . . . . . . . . . . . . . . . . . . . . 91

6.4 .1 Clinical relevance . . . . . . . . . . . . . . . . . . . . . 91

6.4 .2 Technical insights . . . . . . . . . . . . . . . . . . 93

6.4 .3 Limitations . . . . . . . . . . . . . . . . . . . . . 94

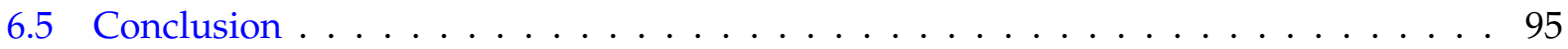

7 A Bayesian method for inference of effective connectivity in brain networks for detect$\begin{array}{ll}\text { ing the Mozart effect } & 97\end{array}$

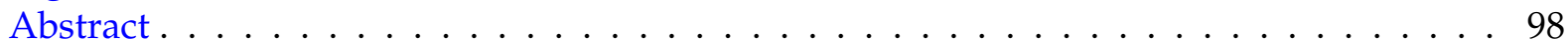

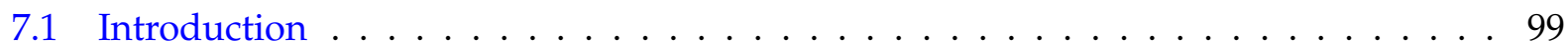

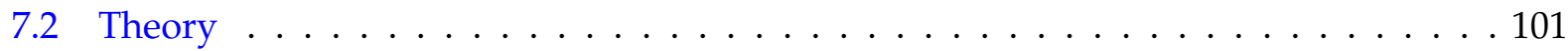

7.2.1 Dynamic network model of brain network connectivity . . . . . . . . . . . 101

7.2 .2 Directed graph . . . . . . . . . . . . . . . . . . . . 102

7.3 Materials . . . . . . . . . . . . . . . . . . . . 102

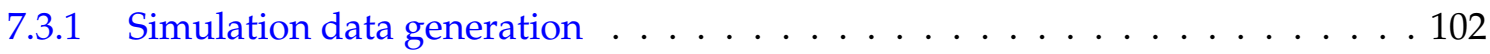

7.3.2 Mozart effect study . . . . . . . . . . . . . . . . . . . . . . . 103

Mozart's Sonata K448 experiment . . . . . . . . . . . . . . . . . . . 103

Acquisition . . . . . . . . . . . . . . . . . . 104

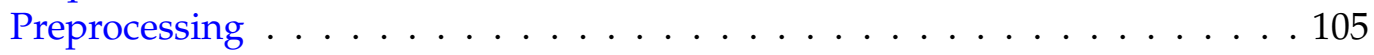

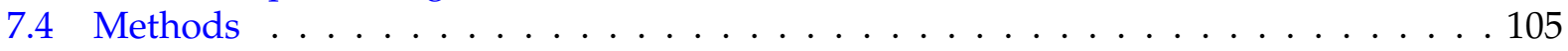

7.4.1 Granger-causality analysis . . . . . . . . . . . . . . . . . . 106

7.4 .2 Bayesian topology identification . . . . . . . . . . . . 107

7.4.3 Evaluation of methods in simulation . . . . . . . . . . . . . . . . 108

7.4 .4 Bayesian group hypothesis test . . . . . . . . . . . . . . . . 108

7.4 .5 Validation of ICA time series AR models . . . . . . . . . . . . . . . . . 110

7.4.6 Inference of the existence of a Mozart effect . . . . . . . . . . . . . . . . . 110

Inference based on listening duration $\ldots \ldots \ldots 111$

7.5 Results . . . . . . . . . . . . . . . . . . . . . . . 112

7.5.1 Evaluation of methods in simulation . . . . . . . . . . . . . . . . 112

7.5.2 Inference of the Mozart effect . . . . . . . . . . . . . . . . . . . . 112

7.5 .3 Inference based on listening duration . . . . . . . . . . . . . . . . 114

7.6 Discussion . . . . . . . . . . . . . . . . . . . . . . . . . . . . . . . . . .

8 Discussion and conclusions $\quad 119$

8.1 Comparing causality, coherence, and evolution dynamics . . . . . . . . . . . . 121

8.2 Limitations . . . . . . . . . . . . . . . . . . . . . . . . . . . . . . . . . . . . . . . . . . . .

8.2.1 Preprocessing, parcellation and global regression for replicability . . . . . . . 123

8.2.2 Limitations in the dynamics method analyses . . . . . . . . . . . . . . 124 
8.2.3 The top-down and bottom-up integration issue . . . . . . . . . . 126

8.3 Future directions for neurodynamics in neuropsychiatric disorders . . . . . . . . 126

8.3.1 fMRI, preprocessing, and individual mapping . . . . . . . . . . . . . 126

8.3.2 Multidimensional and neuropsychiatric-specific biomarkers . . . . . . . . . 127

8.3.3 Classifier, improved models and images . . . . . . . . . . . . . . . . . 127

8.4 General conclusions . . . . . . . . . . . . . . . . . . . . 128

$\begin{array}{ll}\text { A Appendix Chapter } 4 & 131\end{array}$

A.1 Multivariate Conditional Granger Causality theory . . . . . . . . . . . . . . 131

A.2 Supporting information of no between-group difference in spatial RSN connectivity 133

A.3 1-back task fMRI protocol . . . . . . . . . . . . . . . . . . . 134

A.3.1 1-back Task description . . . . . . . . . . . . . . . . . . 134

A.3.2 Task procedure . . . . . . . . . . . . . . . . . . 135

A.3.3 Task design . . . . . . . . . . . . . . . . . . 135

B Appendix Chapter 5 137

B.1 Results of all classifier tested in setup 1 (4 features) . . . . . . . . . . . . . . . . 137

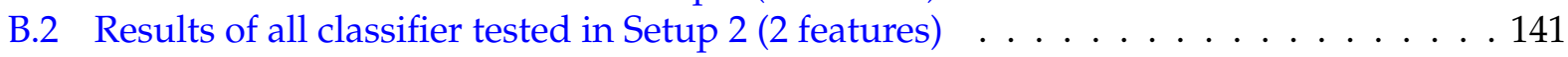

$\begin{array}{ll}\text { C Appendix Chapter 6 } & 145\end{array}$

C.1 Figures of the best feature for each dynamics metric . . . . . . . . . . . . . . . 145

C.2 Feature setups giving the best classification performances . . . . . . . . . . . 147

$\begin{array}{lr}\text { D Appendix Chapter } 7 & 149\end{array}$

D.1 Approximation of hypothesis likelihood marginalization . . . . . . . . . . . . . 149

$\begin{array}{ll}\text { Bibliography } & 151\end{array}$

$\begin{array}{ll}\text { List of publications } & 171\end{array}$

$\begin{array}{ll}\text { Valorisation } & 173\end{array}$

$\begin{array}{ll}\text { Acknowledgements } & 177\end{array}$

$\begin{array}{lr}\text { Curriculum Vitae } & 179\end{array}$ 


\section{List of Figures}

1.1 Systemic visualisation of brain functioning $\ldots \ldots \ldots \ldots \ldots \ldots$

1.2 Static vs dynamic FC for explaining behavioural variances . . . . . . . . . . . . 10

1.3 Visualisations of rs-fMRI-based neurodynamics and their application on neuropsychiatric disorders . . . . . . . . . . . . . . . . . . . . . 12

1.4 Activity time series of four cognitive networks from an ACA patient . . . . . . . 15

1.5 Granger causality example . . . . . . . . . . . . . . . . . . . 17

1.6 Example of a wavelet-coherence $\operatorname{map} \ldots \ldots \ldots \ldots \ldots$

1.7 Schematic description of the EGN model . . . . . . . . . . . . . . . . 20

2.1 Resting-state networks extracted from group-ICA . . . . . . . . . . . . . 30

2.2 Most relevant within-network differences between the groups for RS1 and RS2 . . 31

2.3 Network connectivity differences between ACA and Controls . . . . . . . . . . . . 32

2.4 Significant correlations between cognitive measures and the between-network func-

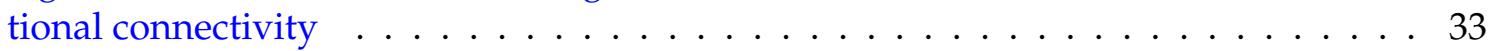

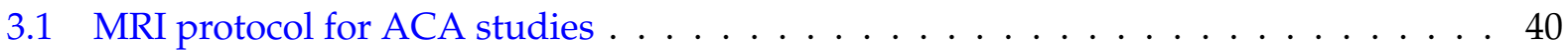

3.2 Networks extracted from group-ICA . . . . . . . . . . . . . . . . . 43

3.3 Pairs of networks that showed differences in GC between ACA and controls . . . . . 44

3.4 Significant correlation between the GC effective connectivity and the psychological deterioration scores . . . . . . . . . . . . . . . . 45

3.5 Summary results of GC in ACA . . . . . . . . . . . . . . . . 46

4.1 Relevant components extracted from the group-level ICA . . . . . . . . . . . . . 58

4.2 Visualisation of the weaker neurodynamic pattern in adolescent with HFA . . . . . 59

5.1 The seven most relevant networks identified in our in-house sample for both groups (ASD and normal controls) . . . . . . . . . . . . . . . . . . . 70

5.2 Continuous wavelet transform upon two resting-state signals . . . . . . . . . . . 71

5.3 Average of time of in-phase coherence in ASD and controls for two RSN pairs . . . 72

5.4 Visualisation of the group-wise RSNs . . . . . . . . . . . . . . 74

6.1 Overview of the methodology . . . . . . . . . . . . . . . . 82

6.2 Brain networks (RSN) time series extraction . . . . . . . . . . . . . . . . . 85

6.3 14 large-scale brain functional resting-state networks (RSNs) used in the study . . 85

6.4 Dynamics feature extraction and statistical analysis . . . . . . . . . . . . . . 87

6.5 Example of \% time of in-phase coherence per group (ACA, Controls) between MVISU and $\mathrm{DMN} \ldots \ldots \ldots \ldots \ldots \ldots$

7.1 Example of a six-node directed graph. . . . . . . . . . . . . . . . . . 102

7.2 Listening duration in hours of all 16 subjects . . . . . . . . . . . . . . . 103

7.3 Spatial maps of the 20 active brain networks in healthy participants of the Mozart study . . . . . . . . . . . . . . . . . . . . 105

7.4 FPR vs TPR of the Bayesian method and Granger-causality analysis graph estimates 111 
7.5 Selection frequencies of four connections of with possible effects of interest $\ldots . .113$

7.6 Brain networks in which the connections were found to have the most important changes in effective connectivity . . . . . . . . . . . . . . . . . 115

A.1 Group mean effect per network . . . . . . . . . . . . . . . . . . . . . . . . . . . 134

A.2 Images displayed for $n$-back task in ASD study . . . . . . . . . . . . . . . . 135

A.3 Design of the 1-back visual task . . . . . . . . . . . . . . . . 136

C.1 Static functional connectivity best feature selection . . . . . . . . . . . . . . . . . 145

C.2 Pairwise Granger causality best feature selection . . . . . . . . . . . . . . . . . . . . 146

C.3 Granger causality degree best feature selection . . . . . . . . . . . . . . . . . 146

C.4 Emulative power best feature selection . . . . . . . . . . . . . . . . . . . . 146 


\section{List of Tables}

2.1 Demographic, clinical and neuropsychological characteristics of the ACA and con-

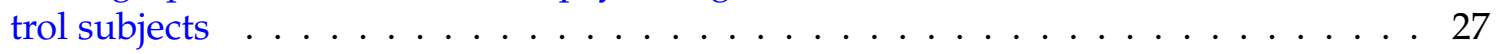

3.1 Demographic, clinical and neuropsychological characteristics of the subjects . . . . 41

4.1 Demographic and descriptive data of ASD and control adolescents . . . . . . . 57

5.1 Demographic and clinical characteristics of the two datasets . . . . . . . . . 66

5.2 Best classifier performances for ASD detection . . . . . . . . . . . . 73

6.1 Demographic, clinical and neuropsychological characteristics of the study partici-

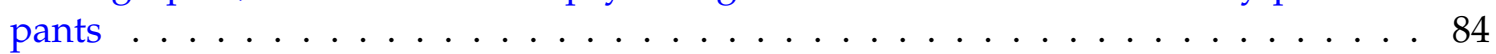

6.2 List of all 86 best features used in the classification analyses . . . . . . . . . . . 89

6.3 Summary of the best classifier performances for each dynamic measures: EGN, WCoh, sFC, and GC . . . . . . . . . . . . . . . . . . . 9 90

6.4 Summary of the best performances of each classifier algorithm: SVM, LDA, KNN, and $\mathrm{DT} \ldots \ldots \ldots \ldots \ldots \ldots \ldots$. . . . . . . . . . . . . . . . . . . . . . . .

7.1 List of all brain networks found in the group ICA decomposition. . . . . . . . . . 104

7.2 Results of the extended Bayesian method on the ICA time series . . . . . . . . . . . 114

7.3 Results of the inference based on the listening duration of subjects from the Bayesian

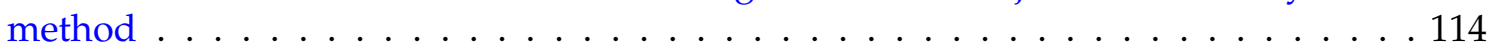

A.1 Smallest FWE-corrected p-values in voxels showing a between-group RSN spatial difference . . . . . . . . . . . . . . . . . . . 133

B.1 Classification performances at all subscales with LDA (setup 1) . . . . . . . . 138

B.2 Classification performances at all subscales with SVM polynomial kernel (setup 1) . 139

B.3 Classification performances at all subscales with SVM rbf kernel (setup 1) . . . . . 140

B.4 Classification performances at all subscales with LDA (setup 2) . . . . . . . . 142

B.5 Classification performances at all subscales with SVM polynomial kernel (setup 2) . 143

B.6 Classification performances at all subscales with SVM rbf kernel (setup 2) . . . . . . 144

C.1 Classifier setups used in Tables 6.3 and 6.4 (Chapter 6$) \ldots \ldots$. . . . . . . . . 147 

Chapter 1

\section{Introduction}





\subsection{Neuropsychiatric disorders and cognition}

\subsubsection{General context of mental diseases}

From all the human organs, the brain is the least understood, albeit being clearly the most important. The human brain contains 86 billion neurons, and around 21 billion of them in the 2-to-4 $\mathrm{mm}$ thick outer (neo-)cortical layer of the brain (Herculano-Houzel, 2009) — where cognition actually occurs. Each of these cortical neurons has an average of 7 thousand synaptic connections, so that our cortex has nearly 150 trillion effective neuronal connections (Drachman, 2005), which is roughly 4 times the number of stars in our galaxy per cubic centimeter of cortex. Therefore, it is not so surprising that this complex information flux and electro-chemical machinery sometimes dysregulates or, in worst cases, completely fails. In such cases, structures or physiology of the brain are disrupted, leading to neurological conditions such as seizures, strokes or movements disorders-to name few. Moreover, it is known that these conditions have strong effect on psychological well-being, and therefore, on the general wellness. Changes in neural functioning can also lead to psychiatric disorders, such as mood or eating disorders, anxiety, depression, dementia and schizophrenia. Patients with neurodevelopmental disorders, such as the autism spectrum disorder (ASD) or the attention deficit / hyperactivity disorder (ADHD) have neurological impairments that lead to clear psychological and behavioural dysfunctions (social impairments and restrictive behaviour in ASD; or attention deficit in ADHD).

In addition, psychosomatic disorders can have the reverse progression, i.e. the condition originates from a traumatic experience, which altered the mind or psychology; then progresses to the biological neural substrate, which in turn spreads and impairs other body parts. Such examples are stress, anxiety, and (some of) the eating and sleep-wake disorders. Post-traumatic stress disorder (PTSD), and major depressive disorder (MDD) are also representations of such psychiatric disorders with psychosomatic symptoms (American Psychiatric Association, 2013). In general, when the neurobiological basis of a psychiatric disease is not well defined-as for most of the mental conditions-finding the origin of the disorder is similar to the egg-chicken problem. Consequently, the evaluation of these neuropsychiatric disorders might reside in the positive, but detrimental, feedback loop, i.e. psychiatric problems worsen the neural dynamics, which then loop back to worsen the psychology of the patient, and so on.

For centuries, philosophers and scientists have tried to shed light on this feedback loop, i.e. the bi-directional neurological-psychological bridge, or the mind-body gap. This thesis tries modestly to investigate this gap using neuroimaging and functional MRI (fMRI). Since the mind rises from gene expressions and neuronal ion channels, and emerges into thoughts involving large brain networks communicating with each other, the focus in this thesis is on the neurodynamics, i.e. the brain neural activity dynamics. These brain dynamics can be evaluated at different levels or scales, but since we want to relate them to slow behavioural activity, we investigate specifically the neurodynamics of large-scale cognitive networks (by means of fMRI). Understanding this mindbody gap, and its mechanism of action when impaired, can lead to better diagnosis and prognosis of mental disorders and to more effective and better targeted treatments at the neural network level, as well as at the psychological level.

To clarify the above statements, Figure 1.1 depicts a schematic representation of the distinction between psychiatric and neurological conditions, and the levels of connectivity at which neuropsychiatric diseases affect the brain. This schematic view of the brain functioning helps to categorise neuropsychiatric diseases. On the one hand, there are psychiatric disorders, such as those associated with anxiety: generalized anxiety disorder, panic disorder, obsessive-compulsive disorder, phobias and social anxiety disorders-in these disorders the abnormal neural substrate is mostly unknown. On the other hand, there are clear neurological conditions, such as traumatic brain 
a

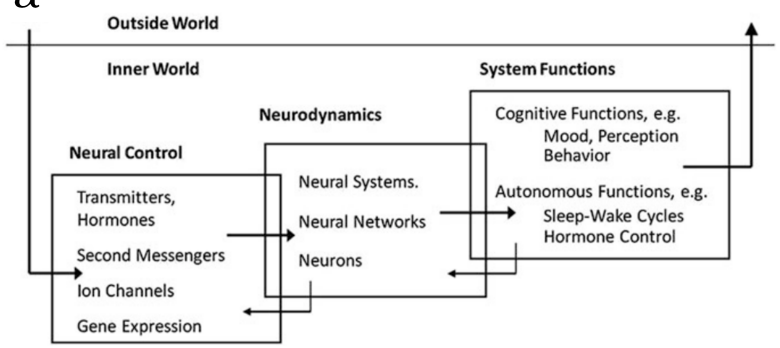

$\mathrm{b}$

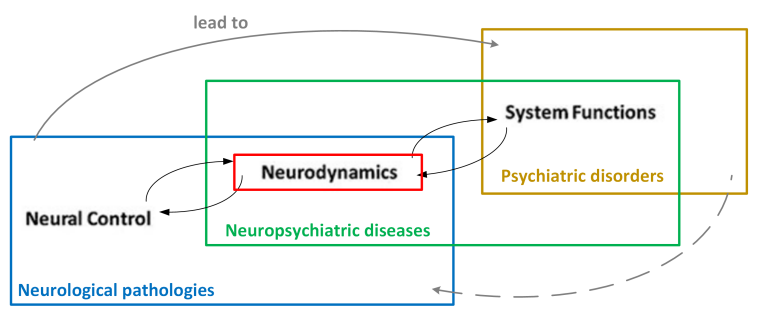

FIGURE 1.1: systemic visualisation of brain functioning. (a) Representation of the different levels of the brain system and their interaction with the outside world-from ion channels and gene expression to cognition and behaviour-and their feedback loops, i.e. top-down and bottom-up processes; (b) schematic representation of the affected levels in the bottom-up and top-down brain connectivity pathways for neurological (blue), psychiatric (yellow) and neuropsychiatric (green) disorders. Neurological illnesses usually lead to psychiatric disorders (solid grey arrow). In some cases, such as in psychosomatic disorders and in pathological feedback loops, psychiatric issues can lead to neurological pathologies (dashed grey arrows). Figure adapted from the figure of Chapter 22 in Wang et al., 2015. The field of neurodynamics (red-squared) is the focus of this thesis.

injury, post-stroke epilepsy and Alzheimer's disease-in those diseases, the neural basis of the impairments are better known. And, in the middle of the spectrum, there are other conditions such as mood disorders (bipolar, depression), and psychotic disorders (e.g. schizophrenia and substance abuse disorders), which lie perfectly in our definition of neuropsychiatric disorders. Child and adolescent developmental brain disorders (e.g. ASD), sleep and wake disorders, as well as trauma-related disorders (e.g. PTSD) also classify as neuropsychiatric disorders with clear psychological impairments, but less obvious neurological implications.

The degenerative process of ageing itself can be viewed as neuropsychiatric, since naturally, the brain physiology tends to be affected by the ageing of brain cells and myelin degradation, which subsequently impair brain functions and cognition (psychology). Other neurological conditions, usually degenerative, are also direct consequences of this ageing effect and degradation of neural cells. Two important examples of these ageing-related neurological conditions are Alzheimer's and Parkinson's diseases (AD, PD). Since the progression of these diseases has major negative impacts on cognitive- and motor-control abilities, they can be classified as neuropsychiatric disorders.

\subsubsection{Cognitive changes in neuropsychiatric diseases}

In this thesis, we focus on neuropsychiatric diseases that affect substantially cognitive abilities. In case of Parkinson's and Alzheimer's diseases (PD, AD), reports of cognitive impairments have been increasingly published (Roheger, Kalbe, and Liepelt-Scarfone, 2018; Zhou et al., 2017). The number of demented patients with clear cognitive dysfunctions, and of high prevalence in those neurodegenerative diseases, is also increasing as population ages (Aarsland, Zaccai, and Brayne, 2005). In a less malignant way, ageing itself is associated with cognitive decline, as already reported in the 90's (Levy, 1994). However, if the cognitive decline is greater than expected for an individual's age and education level, it is defined as mild cognitive impairment syndrome-which is prevalent in around $10 \%$ of adults older than 65 years of age, wherein half will progress to dementia within 5 years (Gauthier et al., 2006). The cognitive impairments or declines in neuropsychiatric disorders can affect episodic memory, fluid intelligence, and processing speed. With the 
exception of the neurodegenerative disorders (AD, PD and dementia) language processing and long-term memory, i.e. crystallized intelligence, are usually less affected.

Impairments in cognition are also presented by the psychiatric conditions of schizophrenia, and the bipolar disorder. In schizophrenia, disorganized speech, false beliefs (e.g. hearing voices), reduced social engagement and emotional expression, and lack of motivation, are markers of cognitive dysfunctions. In case of bipolar disorders, mood swings with emotional highs and lows-often linked to depression-make patients feel sad and hopeless, which in turns reduces cognitive abilities in social engagement and emotional resilience. Regarding epilepsy important psychiatric comorbidity usually appears too: due to the localized seizures affecting cognitive function of the respective areas (Elger, Helmstaedter, and Kurthen, 2004); or because of heavy use of anti-epileptic drugs, increasing the risk of dementia, and its detrimental effect on cognition (Taipale et al., 2018). Frequent seizures and drug-resistant epilepsies often lead to depression, specifically, $30-50 \%$ of patients with refractory epilepsy have major depression (Gilliam et al., 2004). This is an important issue to address since the cognitive decline and brain health of elderly persons with chronic epilepsy had long been neglected (Hermann et al., 2008).

Since most of the aforementioned mental conditions are part of the neuropsychiatric spectrum, and it is clearly impossible to cover them all in one book, we focus in this thesis on two conditions: ASD, a well-known and long-studied neurodevelopmental condition that impairs social cognition, the sense of self, and the behaviour; and the accelerated cognitive ageing (ACA) phenomenon in epilepsy, a mental condition with no clear structural damage, but showing symptomatic impairments in fluid cognition, resembling those observed in 'normal' /'healthy' ageing - but at higher pace. Finally, in the will to help further explore possible treatment in case of cognitive decline or impairments, we study along the power of a non-invasive music-based therapy on healthy young adults. This study could further help new forms of treatment to improve, for instance, cognition in terms of fluid cognition and improved attention. More details on types of treatment for helping cognitively impaired patients are given in the coming subsections.

\subsubsection{Helping diagnosis and treatment}

In most neuropsychiatric conditions, the disorder occurs and is symptomatic at the psychological level; and since the biological origins are unclear, the diagnoses of these conditions are often only made by assessing the psychology of the patients. For example, performance scores such as IQ test and working memory scores are markers of cognitive impairments in MCI. However, some diagnoses are made through interviews, and need a more subtle-and often more subjective-evaluation. This is the case for autism that is assessed through the Autism Diagnostic Observation Schedule (ADOS) or the Autism Diagnostic Interview-Revised (ADI-R) scores. This is why there is a clear need for developing more objective computer-based supportive diagnostic tool, using brain imaging and recording techniques such as electro-, magneto-encephalography (EEG, MEG), and functional magnetic resonance imaging (fMRI). And the attempt to shed light (by means of neuroimaging techniques) on the bridge between neurology and psychology to help diagnoses is realistic. Indeed, psychoradiology has recently been a new field of research (Huang et al., 2019; Lui et al., 2016).

In addition to being difficult or at least long to diagnose, neuropsychiatric disorders are most of the time hard to treat. Pharmacological drugs are one of the solutions for treating partially these psychiatric symptoms. Treating the psyche (psychotherapy) through behavioural training, e.g. with cognitive behavioural therapy (CBT), can also lead to improvement in brain functioning. CBT has been proven to work and have long-term effects on neurodevelopmental disorders such as autism, and ADHD, for instance (Pan et al., 2019; Sofronoff, Attwood, and Hinton, 2005). 
Remarkable work has also been done in PTSD, OCD, as well as MDD (Cuijpers et al., 2009; Dis et al., 2020). More recently, another method of personalised behavioural training has been developed: the neurofeedback technique. This approach has great potential in clinical practice where the abovementioned treatments are weakly effective (Yamada et al., 2017); and it can be performed in real time with fMRI (Heunis et al., 2018)—showing direct and short-term treatment effects. Finally, more advance techniques such as deep brain stimulations or the less invasive transcranial stimulations, can also be used for treating neuropsychiatric disorders. For example, repetitive transcranial magnetic stimulation has already been utilised in clinics for treating MDD (Klooster, 2019).

Overall, these therapeutic methods to control and reduce symptoms of neuropsychiatric disorders will clearly benefit from netter pictures of how the (abnormal) brain works before and after treatment; showing whether or not these therapies have long-term effects. In that regard, we want to show in this thesis that more dynamic (than static) evaluations of the pathological brain processing can help developing the aforementioned treatment protocols and techniques even further. Our goal is therefore to broaden the field of neuroimaging to major neuropsychiatric disorders, such as autism or pathological cognitive ageing so that psychoradiology becomes a major tool in assisting clinicians to objectively diagnose, prognose, and monitor mental conditions and treatment effects. This is why this thesis focuses on brain functioning and dynamics patterns using fMRI-by investigating novel image processing methods and their contribution to psychoradiology.

\subsection{The brain dynamics in fMRI}

\subsubsection{Why functional MRI?}

Neurodynamics is highly important, and might be the only robust biomarker for diagnosing some of the neuropsychiatric disorders. At the neural level, brain dynamics can be captured by use of electroencephalography (EEG) or more invasively with electrocorticography (ECoG). On the other hand, for higher level of brain dynamics, i.e. dynamics in activation of large-scale cognitive/behavioural networks, functional MRI (fMRI) is the most preferred method. The interplay between such brain network activities, even at rest, creates brain states (Billings et al., 2017). In the latter paper the authors conclude that the resting brain actively pursues a range of distinctive states from those adopted during explicit tasks. The realisation of both resting and task-active states involves large-scale, and often phase-locked coordination among multiple brain regions at particular frequencies. To describe brain states, one needs: spatial functional networks (formed by multiple brain regions), a temporal timeframe to interplay, and some rules to obey. Also, the discretisation of these properties is important, i.e. the size of the networks, and their temporal and spectral resolutions of activity. This is usually arbitrary chosen depending on the goal set by the investigators. Here, in this thesis, we will focus on large-scale networks-around 10-20 networks forming the full brain-using fMRI. "Spontaneous dynamics show predictive value over individual cognitive profile and constitute a potential marker in neurological and psychiatric condition" (Madeo et al., 2017). Though, how to define a model for such spontaneous dynamics? At the neural level, the best non-invasive method to capture the brain dynamics is probably the EEG, which give a high temporal resolution. This technique has been used for decades to detect sleep phases (Lazar, Lazar, and Dijk, 2015), epileptic seizures, and their effect on cognitive function (Aldenkamp and Arends, 2004), or even neurophysiological information in MDD (Cook et al., 2014). EEG analyses provide us with the generalised brain waves that govern our mental states, behaviour and action, ranging from $0.5 \mathrm{~Hz}$ to more than $30 \mathrm{~Hz}$. Even though these brain waves or rhythms have been shown to be neurophysiological signatures of higher-level (slower) cognitive network activity (Mantini et al., 2007), it is not fully known how their distribution over 
the cerebrum creates, inhibits or maintains thoughts, attention and behaviour. Therefore, analyzing directly the slower brain fluctuations $(<0.5 \mathrm{~Hz})$ can help observing abnormality in behaviour and cognition. FMRI allows the extraction of such low frequency signals, the blood-oxygen-leveldependent (BOLD) signals. In 'classic' 3T MRI scanners, an fMRI scan would have a usual informative signal ranging from $0.25 \mathrm{~Hz}$ to $0.008 \mathrm{~Hz}$, which can capture wavelength of 4 to $100 \mathrm{~s}$ periods, i.e. slow fluctuation (Biswal et al., 1995). Therefore, fMRI can detect dynamics at higher temporal scale (in seconds, not milliseconds) that captures more global brain states and cognition than the underlying generative faster brain waves seen by EEG. Another benefit of fMRI is the higher spatial resolution than EEG. fMRI can also provide signals from tissue lying deeper in brain-it is not limited by the distance sensor-tissue. Thus, we get a better location of dynamic connections, which can help the development of future localised treatment, or even assist surgical procedures, for treating neuropsychiatric disorders such as autism, schizophrenia, Alzheimer's disease and epilepsy (Du, Fu, and Calhoun, 2018; Zhou et al., 2010). As mentioned before, the diagnosis of neuropsychiatric disorders through image-based neurological assessment has not yet been fully exploited. However, functional MRI and its derived brain functional connectivity are really promising-yet challenging-for diagnosing and even predicting treatment response of brain disorders such as schizophrenia, bipolar disorder, ASD, ADHD, AD and MCI, as shown in the review of (Du, Fu, and Calhoun, 2018). In technical terms, diagnosing a disorder is classifying (detecting) it as such. And classification relies on features, i.e. biological or physiological information/markers that allow separation (healthy / non-healthy). Since features are biological by nature, we simply call them biomarkers. One of the most challenging problems to classify mental disorders is to find an accurate and reproducible (robust) biomarker. Brain dynamics using fMRI might be an answer to the pursuit of accurate and insightful psychoradiological markers.

\subsubsection{Structural and functional connectivity as neurobiomarkers}

MRI-based biomarkers have already been described for numerous neurological conditions. For instance, a white stain in a T2-weighted image is a clear sign of a white matter lesion, which presumes vascular origins, and is commonly found in older subjects who had a stroke or who suffer from dementia (Wardlaw, Valdés Hernández, and Muñoz-Maniega, 2015). Other examples of structural biomarkers are dark spots on the brain grey matter visible in a susceptibility weighted image (SWI). These would tell radiologists that the patient has microbleeds, which can appear in concussed individuals-even though traumatic head injuries are still confusing neurologists (Sharp and Jenkins, 2015). Also, AD leads to clear structural deterioration, such as hypertrophy of grey matter in many cortices, or abnormal white matter integrity in several brain parts, as seen with diffusion weighted imaging (Teipel et al., 2012). A smaller hippocampus turned out to be a relevant marker too-in major depression (Schmaal et al., 2016). In schizophrenia, structural abnormality has also been found: an excessive extracellular volumes (Pasternak et al., 2012) or a lower fractional anisotropy within cerebellar tracts and the left anterior thalamic radiation can reveal a possible onset of the disorder (Mamah et al., 2019; Pasternak et al., 2012). However, these neurobiomarkers are usually specific to the neurological impairments. The problem is that these structural impairments are still only tested after the psychological symptoms are revealed, hence hardly screened and treated before the onset of the psychopathology.

The biomarkers described above are relevant for neurological conditions. However, in most neuropsychiatric disorders, clear structural biomarkers are not well-defined. Hence, looking at the brain functioning or physiology is becoming a priority. In autism, even though strong behavioural dysfunctions are present, no brain imaging technique is so far used in clinical practice to objectively diagnose the condition, despite a variety of image-based (rs-fMRI) evidences/markers of the condition (Barttfeld et al., 2012; Bos et al., 2014; Douw et al., 2016; Lau, Leung, and Lau, 2019; 
Monk et al., 2009; Nomi and Uddin, 2015; Redcay et al., 2013). Similarly, in spite of the intensive research in MDD, biomarkers are limited to hypo-activity in bilateral amygdala, and left anterior insula (Veer et al., 2010), or the subgenual and thalamic hyper-connectivity with the default-mode network (Greicius et al., 2007). Other distinct abnormal patterns have been identified that are related to the severity of specific symptoms in other neuropsychiatric disorders. Examples of such illness-specific alterations include the hypoactivity of ventral medial prefrontal regions in patients with schizophrenia, hypoactivity of the anterior cingulate cortex in patients with ADHD, and hyperactivity of the amygdala in patients with PTSD or anxiety disorders (Lui et al., 2016). These are examples of biomarkers of neuropsychiatric disorders that can be extracted with fMRI. However, resting-state (rs)-fMRI is not yet used in clinical practice because of the issue with ensuring reliability and reproducibility, and standardisation of the used metrics ( $\mathrm{O}^{\prime}$ Connor and Zeffiro, 2019). This improvement in reliability might be better achieved using rs-fMRI dynamic metrics, as seen in the next section.

\subsubsection{Resting-state-fMRI and the benefit of spontaneous dynamics}

There is growing evidence that spontaneous brain activity gives insight on brain pathologies (Liégeois et al., 2019; Rasero et al., 2018). Task-based fMRI evoking brain activity can surely be insightful too, but are often more complex to design and may be biased by non-responders (to the task), which is often the case for patients with neuropsychiatric disorders (Zhou et al., 2010). Conversely, if the task is well executed in the patient group, it can be harder to distinguish them from healthy participants, since the pattern of brain activity would be very similar; which then lead to nonsignificant differences in brain activation in task-based fMRI group comparison studies. Therefore, assessing the resting brain and its patterns of activity might be more reliable and preferred over task-evoked activity, especially for neuropsychiatric conditions (Fox and Raichle, 2007). Overall, nearly 10000 papers on rs-fMRI currently listed in PubMed, and an atypical resting-state connectivity has been demonstrated in a wide range of neuropsychiatric disorders, including epilepsy, schizophrenia, attention deficit hyperactivity disorder, Alzheimer's disease, stroke, and traumatic brain injury ( $\mathrm{O}^{\prime}$ Connor and Zeffiro, 2019). Finally, the correspondence between the resting-state network (RSN) activations and behaviour, and the (potential) use of these RSNs as brain image biomarkers, has been extensively demonstrated (Abraham et al., 2016; Choe et al., 2015; Damoiseaux et al., 2008; Du, Fu, and Calhoun, 2018; Laird et al., 2011; Smith et al., 2009a; Zhou et al., 2010). Nevertheless, static measurement of brain network activity might not suffice to describe the mechanism of action of neuropsychiatric diseases. In the next paragraph the benefits of dynamic over static functional connectivity in the brain resting states are explained.

Sometimes, static measures of brain activation are not enough or less accurate than more dynamic measurements. As mentioned earlier in this introduction, schizophrenia for instance can be detected by observing hypoactivation in ventro-medial prefontal cortex (Zhou et al., 2010). In this review the authors show that other research groups found increased functional connectivity through assessment of the amplitude of low-frequency fluctuation in the same prefrontal cortex (Hoptman et al., 2010). These inconsistencies of the results of networks or regions of interests (ROIs) being hypo- or hyperactivated at rest are also found in autism (Anderson, Ferguson, and Nielsen, 2013; Kana et al., 2014; Müller et al., 2011). The contradictory results that appear in static functional connectivity (sFC) analyses of schizophrenia, autism, but also in ADHD and AD rsfMRI studies (see summary tables of $\mathrm{Du}, \mathrm{Fu}$, and Calhoun, 2018) are most probably due to the divergence in the methods used in the studies (seed-based or ICA decompositions; more details in the next subsection) and the derived metric applied on the time series (e.g. correlations between seed time-series or power analysis of low-frequency components). 
In order to counter that inconsistency in rs-fMRI findings, and strengthen reliability of the underlying mechanism of action of the neuropsychiatric disorders, dynamic functional connectivity (dFC) metric might be used. For instance, in case of schizophrenia, instead of the general hypoactivation in prefrontal cortex, measuring a dwelling time in a specific brain state greatly helps distinguishing the disease (Damaraju et al., 2014). This dwelling time is, indeed, a neurodynamic metric of rs-fMRI scans as shown in Figure 1.3.c. With respect to autism, limited literature shows that dynamic functional connectivity (dFC) might also help describing the disease, and help the diagnosis (Uddin et al., 2015). Finally, study of ageing and its cognitive decline can benefit from fMRI dynamic analyses, since an effective connectivity (explained in the next section) correlates with the affect processing during emotional tasks in elderly (Sala-Llonch, Bartrés-Faz, and Junqué, 2015). Yet, the evidence of changes in dynamics during cognitive task is limited. One paper has linked cognitive processing and ageing using the dynamics metric of effective connectivity (Tsvetanov et al., 2016).

In a review article, Liégois et al. proved that there is an overall benefit of rs-fMRI dynamic analyses over sFC analyses for assessing and understanding people's behaviour and cognition (Liégeois et al., 2019). Indeed, in healthy subjects, behavioural measurements such as perceptual reasoning index or processing speed (measures of fluid intelligence) are better explained through $\mathrm{dFC}$ than static connectivity. Other between-subject variance in performances of cognitive tasks such as an $\mathrm{N}$-back task (working memory test) or emotion face matching are also better explained using dynamic metrics of fMRI. However, these dynamic metrics are less relevant for more subjective selfreported psychological measurements, such as a loneliness, happiness or perceived stress tests, for instance. Therefore, overall, the best type of analyses is the combined assessment of the dFC and the sFC to explain behavioural variances as shown in Figure 1.2. It is worth noting that this work has been evaluated on healthy subjects and needs further investigation in case of mental disorders. This is why, in this thesis, we focus on brain dynamics, or neurodynamics, in neuropsychiatric disorders. Before describing our novel approaches for $\mathrm{dFC}$ analyses in resting-state fMRI, the well-known sFC methods and the more recently applied dFC techniques are described in the next subsections.

\subsubsection{Classical models (static FC)}

The main use of rs-fMRI in clinical studies is to derive from the blood-oxygen-level-dependent (BOLD) signals co-activation (spatial) patterns, i.e. network of (spontaneous) activation. To do so, Pearson's correlation is calculated between time series. These time series can be the BOLD signals of each voxel (3D smallest spatial unit of the fMRI data), or an average of them in a region of interest (ROI; aggregate of voxels). This cross-correlation technique is known as seed-based FC analysis of fMRI. Once the spatial networks or correlation matrices (between all seeds) are created, summary statistics, e.g. 2-sample t-tests, can infer discrepancy between groups, in case of crosssectional and case-control study. This discrepancy between a group of patients and a control group is then visible at the entries of the correlation (adjacency) matrix, which displays the abnormal FC with hyper- or hypo-activity. In a network representation, a significant difference in the correlation matrix shows the abnormal edges (disconnected nodes, for instance). However, this approach to inferring group difference can be quite noisy and underpowered since the ROIs or seeds must be selected a priori, and drastic inter-subject variability in the correlation matrix (activation maps) occurs. Nevertheless, in case of subject-specific intervention, such as for a surgery, this seed-based method might be preferred.

In order to counter this a priori seed-selection problem for group comparison, independent component analysis (ICA) is a good and robust approach to extracting brain networks (Beckmann et al., 2005; Choe et al., 2015; Laird et al., 2011; Wang, Zeng, and Chen, 2012). This data-driven 


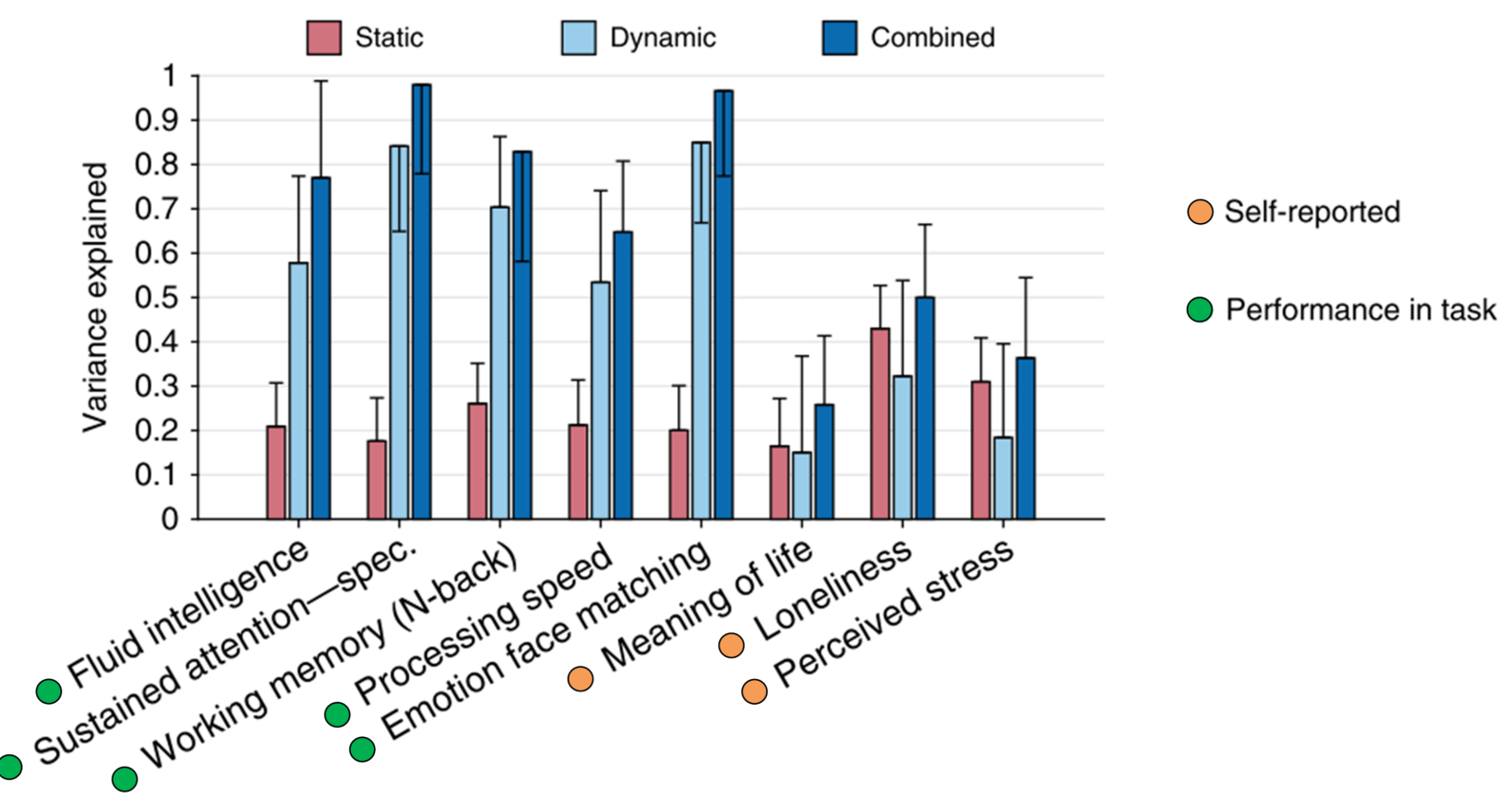

FIGURE 1.2: Static vs dynamic FC for explaining behavioural variances. The barplots show the benefit of dynamic (and combined dynamic-static) over static analysis for explaining variances in task-performance (green) and self-reported (orange) measures. Figure adapted from Liégeois et al., 2019.

approach has the advantages to have no a priori on the size and location of the networks. Only the number of components (or networks) is usually arbitrary selected, but can also be statistically and automatically defined. Also, ICA does not need global signal regression in general since the methods can extract 'noisy' networks originating from these global signals (CSF or white-matter artefactual activation), scanner artefacts, and participants head movements or physiological noise (heart-beat, breathing). This method can even be robust to non-stationarity if the Infomax algorithm is used (Bell and Sejnowski, 1995; Esposito et al., 2002). Note that in this thesis, all chapters we consider independent components (ICs) or networks of activation and their time series. Since ICA genuinely splits the brain into networks from statistical modeling, not from predefine seeds or ROIs, results (in rs-fMRI) are normally less misleading for group difference inferences. Like in seed-based FC analyses, after obtaining subject-specific activation maps or ICs, one can group-wise compare their extent and strength of activation with basic parametric statistics, such as 2-sample t-tests upon all voxels forming those maps.

From these IC or seed time series, other static FC measurements can be derived from rs-fMRI. For instance, once the activation map is extracted, the frequency content of an ROI or a network can be extracted- using the ROI- or network- time-series, the index Amplitude of Low-Frequency Fluctuation (ALFF) can be evaluated. ALFF, in which the square root of power spectrum is integrated in a low-frequency range is said to detect the regional intensity of spontaneous fluctuations in BOLD signal, and can be used for such SFC analyses. Regarding brain diseases it seems that only ADHD children (Zang et al., 2007), schizophrenia (Hoptman et al., 2010), and more recently MDD (Sambataro et al., 2017) have undergone this type of resting-state analyses.

Other examples of static metrics derived from static FC are the properties of network topology in graph theory. Indeed, a FC correlation matrix (from seed-based analysis) can be turned into graphs; and by using graph theory and the spatial distribution of the nodes (seed/voxels) and edges (correlation values), properties of the graph such as small-worldness, efficiency, nodal centrality, or modularity can be derived (Wang, 2010). This technique has been applied on several 
neuropsychiatric disorders, such as autism (Keown et al., 2017), AD (Buckner et al., 2009), epilepsy (Liao et al., 2010), and schizophrenia (Liu et al., 2008). These network-graph metrics can infer the notion of integration and segregation of brain networks, which have implication in explaining ageing brain connectivity, as depicted in Figure 1.3.a. Graph theory analysis of network functional connectivity has also shown great results in the diagnosis of autism, leading to $92 \%$ accuracy in classifying the disorder, using the rs-fMRI-based global and local network efficiency metric (Sadeghi et al., 2017). In the more traditional seed-based functional connectivity analyses accuracy of classifying autism hardly reaches $80 \%$ (Du, Fu, and Calhoun, 2018; Plitt, Barnes, and Martin, 2015). Using a multimodal approach, e.g. rs-fMRI with diffusion tensor imaging (DTI), or a most sophisticated classifier algorithms (e.g. probabilistic neural network), seems to help reaching the classification accuracy over 90\% (Deshpande et al., 2013; Iidaka, 2015).

Finally, the abovementioned accuracy values for diagnosing autism holds for other, more often studied, neuropsychiatric disorders, such as schizophrenia and bipolar diseases, ADHD, AD, and MCI (Du, Fu, and Calhoun, 2018). To that regard, another way to increase accuracy in diagnosing these diseases might reside in assessing not the static FC, but the network dynamics FC-especially when mixed results of hypo- or hyper-activity are present (e.g. in autism). The next subsection presents several newly developed dynamic modeling methods for rs-fMRI, which can help the diagnostic and prognostic processes in neuropsychiatry.

\subsubsection{Dynamics models (dynamic FC)}

Dynamics is defined as the forces or properties which stimulate growth, development, or change within a system or process. Fundamentally, those changes involve time. Therefore, unlike static connectivity, dynamic connectivity assesses changes in connectivity over time. More concretely, two options to assess dynamics in the brain are possible. The first option is to use known static connectivity measurement, e.g. correlation, over a time window, then to slide the window and repeat the measurement. With this process, one can extract a new (dynamic) signal that shows the variation of the connectivity over time. The other option is to get a static measurement (average over the full period of time of an fMRI scan session) but from a dynamic model, i.e. a summary metric describing brain dynamics.

The first option has been implemented based on the static model of brain functional connectomes, i.e. the complete topology of functional connectivity (Insabato, Deco, and Gilson, 2019). Indeed, it has been shown that brain connectomics can vary throughout an fMRI scan session: participants do no experience one long, continuous connectivity state through the scanning, but several (Abrol et al., 2016). Undoubtedly these temporal variations of neural activity are critical aspects of cognition and behaviour (Gonzalez-Castillo et al., 2019; Hutchison et al., 2013). Consequently, dynamic functional connectivity ( $\mathrm{dFC}$ ) analysis can provide a better description of mental disorders, which mainly affect cognition and behaviour of the patients. For describing this dFC in the brain of participants, the sliding-window of Pearson correlation matrices is most commonly used (Preti, Bolton, and Van De Ville, 2017). If the dFC is applied on the full brain (i.e. all voxels from the fMRI scan), it is coined the chronnectome (Abrol et al., 2016; Calhoun and Adali, 2016; Nomi et al., 2016). When clustering common spatio-temporal pattern of activity, e.g. with k-means, the dFC, or chronnectome, gives rise to common brain states recurrent as rest (Allen et al., 2014; Calhoun and Adali, 2016; Calhoun et al., 2014; Leonardi et al., 2014; Nomi et al., 2016). These brain states have been shown to be reliably occurring across large samples of data (Abrol et al., 2016; Choe et al., 2017). Even though caution is to be taken regarding the reliability of summary measures derived from these brain states (Choe et al., 2017), the dwelling times in some of these mental states are good descriptors for schizophrenia (Damaraju et al., 2014). Examples of such brain-state representation of brain dynamics, and the derived dwelling times are depicted in Figure 1.3.c. 


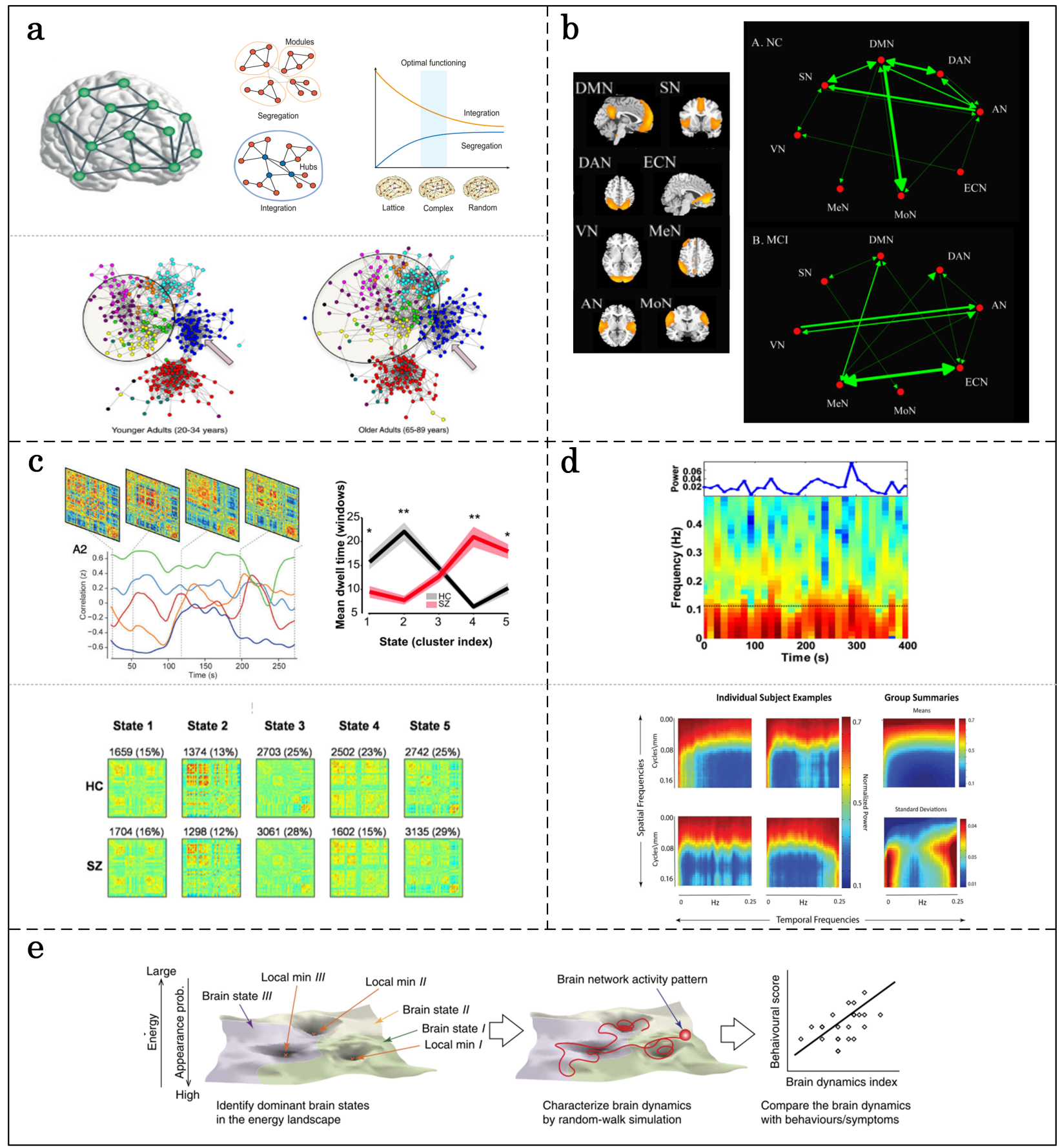

FIGURE 1.3: Visualisations of rs-fMRI-based static FC. (a) and neurodynamics (b-e), and their application on neuropsychiatric disorders. (a) Graph theory approach, showing that using static functional connectivity (FC) matrix, spontaneous brain activity topologically forms networks that segregate into modules or integrate into hubs (top), which can be used to graphically display population age differences (bottom), with increased age associated with decreased segregation between modules (circles), and within hubs (e.g. in the visual system highlighted by the arrows). bottom left - young population; bottom right - elderly. (b) Schematic representation of effective (directed) connectivity between well-known large-scale RSNs (ICA-extracted) that can group-wise distinguish MCI patients from controls. (c) Sliding-window approach of FC matrix (top-left), for spatiotemporal analysis of FC with temporal k-means clustering to visualise brain states (bottom), allowing discrimination of schizophrenic patients as their mean dwelling time into each of these states significantly differs from healthy controls (red and black line plot; top-right). (d) Spectrotemporal visualisation of thalamic activity at rest in a healthy subject using fMRI (top); or a more advanced spatiotemporal approach that spectrally profiles brain network activity, proven to have great diagnostic potential (e.g. effect of ageing) (bottom). (e) A combination of non-linear dynamics applied onto state-based profiling, showing the pathway of brain activity dynamics throughout the energy landscapes of brain states, which allows characterisations of autistic brain processing at rest. Figure adapted from (a) (Chan et al., 2014; Deco and Kringelbach, 2014; Fornito, Zalesky, and Breakspear, 2015); (b) (Zhenyu Liu et al., 2012); (c)(Damaraju et al., 2014; Rashid et al., 2014); (d) (Liu et al., 2018; Mezer et al., 2009; Miller et al., 2015); (e) (Watanabe and Rees, 2017). 
Similar dynamic (sliding-windows) assessment can be performed on other static measurements, such as the frequency spectrum. Indeed, instead of a simple Fourier transform of brain network activity, one can extract the sliding window discrete Fourier transform of the activity, i.e., a spectrogram (spectrum display over time, Figure 1.3.d). This time-frequency analysis can also be performed using the wavelet transform (Chang and Glover, 2010; Yaesoubi et al., 2015). However, we are not aware of such time-frequency dynamics analysis applied on neuropsychiatric disorders. Hence, we evaluate in this thesis the utility of summary measures extracted from waveletcoherence maps (i.e. time-frequency dynamics maps) on adolescents with autism and patients with ACA. More details on wavelet-coherence and our derived dynamics metric are provided in the next section.

Another way of assessing brain dynamics, which gains a lot of interest in brain dynamics imaging, is the evaluation of causality. This is the natural step forward from sFC that uses correlation, i.e. undirected connectivity measurements. Indeed, in a causality model, the directivity and strength of the connectivity are described, hence giving us more information about how much connectivity is measured from a node (of a network; the source) towards another one (the sink/target). These directed causal connections are usually called effective connectivity. Three types of models to describe such connectivity between nodes of a network exist: auto-regressive methods, Bayesianbased models, and structural equations modeling (SEM). The latter, albeit having been the most used methods to infer effective connectivity in the early 2000's, is now considered limited in its use for model-based brain connectivity. Indeed, since SEM gives rise to acyclic network topology (Friston, 2011) — not representative of the brain dynamic connectivity realm-a hypothesis-driven Bayesian approach, such as dynamic causal modelling (DCM) is often preferred in fMRI studies (Penny et al., 2004). Regarding the auto-regressive techniques, the most popular is the Grangercausality (GC) method (Seth, Barrett, and Barnett, 2015). This is the effective connectivity method we will be using in this thesis (more details on the algorithm in the next section). A detailed comparison of the three main aforementioned methods (GC, SEM, and DCM) for assessing effective connectivity in the brain using fMRI is available in (Friston, Moran, and Seth, 2013; Friston, 2011; Penny et al., 2004). Regarding the role of effective connectivity measurement in neuropsychiatry, some research has been done on MCI (Zhenyu Liu et al., 2012), Figure1.3.b), and depression (Rolls et al., 2018). Maintaining cognition in ageing was also linked to changes in effective connectivity between and within brain networks in healthy participants (Tsvetanov et al., 2016). DCM has also shown an interesting application for real-time application for neurofeedback in a visual-spatial task (Koush et al., 2013), which could be used for helping in deficit of ADHD patients for instance.

A new approach consists in combining effective or dynamic connectivity with graph theory, which resemble study of communication dynamics in networks (Avena-Koenigsberger, Misic, and Sporns, 2018). Other advanced methods with spatio-temporal decomposition and representation through spectral profiling have been recently studied and present interesting potential for diagnosing neuropsychiatric diseases as well (Miller et al., 2015; Figure 1.3.d).

More complex models, assuming the non-linearity of brain dynamic activity, have been tested mainly upon healthy subjects (Churchill et al., 2016; Koppe et al., 2019). Note that it is actually the noise repartition and strength in different parts of the brain than can generate non-linear dynamics in the brain. Non-linear models, such as chaos theory or aperiodic fluctuation modeling, help defining modulation in neural signals, or help describing the transition from a brain state to another as soon as a stimulus (external or internal) is presented. Non-linear dynamics usually arises from scale free or 'fractal' dynamics (Ciuciu et al., 2012; Ciuciu, Abry, and He, 2014), following Lyapunov divergence, or Lorenz strange attractor (Koppe et al., 2019). The Hurst exponents of RSN time series have also been used, and provide insight into the pathological mechanisms of depression (Wei et al., 2013). Chaos theory has even been applied on autism to identify dominant brain states (attractors) and their transient states (bifurcations) within the energy landscape 
(Watanabe and Rees, 2017) of the brain, allowing brain activity in ASD to be distinguished from brain activity in healthy participants, as shown in Figure 1.3.e. These non-linear methods are showing promising results, but with reasonable assumptions the brain dynamics can be simplified into linear equations. Therefore, we base our research on linear models in this thesis.

Finally, an evolutionary game theory-based method has recently been introduced in neuroscience and fMRI analysis: the evolutionary game theory on network approach (Madeo et al., 2017). In this approach, RSN time series are seen as population (of neurons) growth (or activation). This game theory approach uses simple rules of cooperating and/or defecting other players (brain networks). More details on this method are presented in the next section.

Overall, three novel approaches for fMRI analysis, namely Granger causality, wavelet coherence, and evolutionary game theory, are used in this manuscript (Chapter 3 to 6). These techniques will be also compared by use of supervised machine-learning classification methods, in which the best accuracy/predictive results will show us the discriminative power (for neuropsychiatric disorders) of the abovementioned dynamics methods (Chapter 5 and 6). Another method using Bayesian (non-deterministic) modeling to infer effective connectivity will be also assessed (on healthy subjects) and compared to Granger causality (Chapter 7).

\subsection{Overview of the proposed approaches}

We have introduced the context of neuropsychiatric disorders, how they are currently diagnosed and treated. We have also described, in the previous sections of this introduction, the current and promising techniques for estimating dynamic functional connectivity. In this section, the imaging and post-processing techniques we focus on throughout the thesis are specified. We also explain in this section our general method for parcellating the brain of the patients' rs-fMRI data into functional networks and for extracting time-series upon which we apply our novel dynamics metrics.

\subsubsection{Rs-fMRI preprocessing and functional parcellation of the brain}

There is clear evidence that spontaneous brain activity-not evoked one-provides insight on brain pathologies (Rasero et al., 2018). Also, the correspondence between resting-state networks (RSNs) and behaviour, and the use of RSN maps as brain image biomarkers has been extensively demonstrated (Abraham et al., 2016; Choe et al., 2015; Damoiseaux et al., 2008; Du, Fu, and Calhoun, 2018; Smith et al., 2009a; Zhou et al., 2010). Therefore, in this thesis, we restrict the study of neuropsychiatry-related neurodynamics to resting-state (rs-)fMRI data analysis. Regarding neuropsychiatric disorders, the focus of this thesis is put on two types of neuropsychiatric disorders: neurodevelopmental disorder for adolescents with autism spectrum disorder (ASD), and the cognitive decline in the Accelerated Cognitive Ageing (ACA). To get a better idea of the mechanism of action in the brain of these patients and to, the effect of listening to Mozart's music, in terms of changes in neurodynamics, is also studied in our research.

Before diving into the brain dynamics using functional brain networks, their maps and time series, one should be aware that so far there is no prevalent (generalisable to all humans) brain functional atlases (Salehi et al., 2020). Many research groups have developed structural and functional brain atlases with the use of fMRI (Eickhoff, Yeo, and Genon, 2018). The most utilised and referred structural atlas is the Brodmann's classification of brain areas, defined and numbered by the eponym German anatomist. Even though it is quite accurate in delineating important gyri and areas of the brain such as the visual, motor, subcortical glands, auditory and language regions, it 

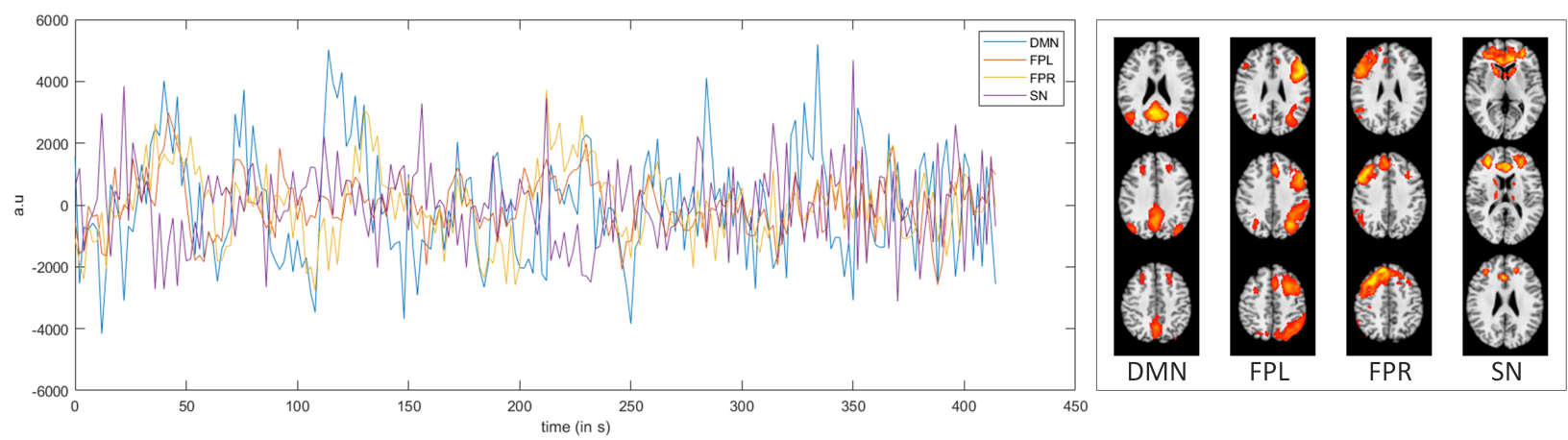

FIGURE 1.4: Activity time series (left) of four cognitive networks (right) from an ACA patient; extracted from a group-ICA applied on his (preprocessed) resting-state fMRI scan followed by dual-regression. DMN - default mode network; FPL - fronto-parietal left network; FPR-fronto-parietal right network; SN - salience network.

is not ideal for parcellating the brain functional areas, which are now known to be spread over the brain into networks. More recently, to allow groups comparison of functional brain network activity, other atlases have been developed. For instance the Functional Imaging in Neuropsychiatric Disorders Lab (FINDLab ${ }^{1}$ created a 90 functional ROIs atlas of the brain (aggregated into 14 large-scale networks) using group-ICA over twenty-seven healthy right-handed subjects (Shirer et al., 2012). At TReNDS ${ }^{2}$ centre (previously MIAlab), Calhoun and his colleagues developed a 28-functional-networks atlases from resting-state fMRI data of 603 healthy adolescents and adults (Allen et al., 2011). Finally, more subject-dependent atlases can be also used, such as the widely applied automated anatomical labeling (AAL) atlas (Tzourio-Mazoyer et al., 2002).

In general, the atlases introduced above are based on finding the consistent functional networks shared by many healthy subjects. There are other atlases created dependent on age (e.g. an atlas for children, Fonov et al., 2011). However, recently those types of group average brain mapping are debated: e.g., Salehi stated in his last paper that there is no single functional atlas even for a single individual, since the functional parcels change with tasks (Salehi et al., 2020).

Taking these specifics into consideration, we adopt a subject-specific functional brain parcellation using group-ICA decompositions followed by a dual regression-back projection to subjectspecific networks - that are study-dependent. This means that the atlases, or brain networks, are data-driven. Nevertheless, the atlas from (Smith et al., 2009a) of the 10 most relevant RSNs is used to validate our choice of relevant resting-state networks. As we assess rs-fMRI, those subjectdependent brain functional atlases should be quite robust to inter-subject variability. An example of four subject-specific cognitive brain networks and their associated time series is depicted in Figure 1.4. these are the type of time series and networks in which we investigate the dynamics in this thesis.

In the following subsections, we introduce the approaches to assess the brain dynamics. The choice of the dynamic modeling methods came from different perspectives and theories of how brain cells forming functional networks interplay together. We summarize them into three domains of dynamics analysis: causality, coherence, and evolution.

\footnotetext{
${ }^{1}$ http://findlab.stanford.edu/research.html

${ }^{2}$ http: //trendscenter.org/
} 


\subsubsection{Causality between brain networks}

Predictability in brain dynamic patterns of activation has always been the grail for neuroimaging researchers and scientists. Such predictability can be seen in causality or effective connectivity. As mentioned earlier, one method to extract causality between signals is the Granger causality approach. Granger causality (GC) was introduced by Granger in 1969 in the field of economics (Granger, 1969). Given two signals $X$ and $Y$ to be analysed, this technique allows to measure how much the past of $X$ (and its information content) can help predict the future of $Y$, rather than just with the past of $Y$ itself (Seth, 2010). This definition of causality can also be conditioned upon other signals in $Z$ (vector of time series), in order to remove possible spurious causality. If we consider the set of time series vectors

where is the target (or sink) variable, the source variable, and the multivariate (conditional signals), then the VAR model can be described as below

$$
\begin{aligned}
& \mathbf{U}_{t}=\left(\begin{array}{l}
\mathbf{X}_{t} \\
\mathbf{Y}_{t} \\
\mathbf{Z}_{t}
\end{array}\right) \\
& \mathbf{X}_{t}=\sum_{k=1}^{P} A_{x x, k} \cdot \mathbf{X}_{t-k}+\sum_{k=1}^{P} A_{x y, k} \cdot \mathbf{Y}_{t-k}+\sum_{k=1}^{P} A_{x z, k} \cdot \mathbf{Z}_{t-k}+\boldsymbol{\varepsilon}_{x, t} \\
& \mathbf{X}_{t}=\sum_{k=1}^{P} A_{x x, k}^{\prime} \cdot \mathbf{X}_{t-k}+\sum_{k=1}^{P} A_{x z, k}^{\prime} \cdot \mathbf{Z}_{t-k}+\boldsymbol{\varepsilon}_{x, t}^{\prime}
\end{aligned}
$$

Where $p$ is the model order, and $A_{i j, k}$ is the regression coefficient between the time series $i$ and $j$ at $\operatorname{lag} k$, and $\varepsilon_{t}$ represents the residuals.

From this VAR modeling of $\mathbf{U}_{t}$, the conditional Granger causality from $\mathbf{Y}_{t}$ to $\mathbf{X}_{t}$ is defined as the likelihood ratio

$$
F_{\mathbf{Y} \rightarrow \mathbf{X}} \equiv \ln \frac{\left|\Sigma_{x x}^{\prime}\right|}{\left|\Sigma_{x x}\right|}
$$

where $\Sigma_{x x}^{\prime}=\operatorname{cov}\left(\varepsilon_{x, t}^{\prime}\right)$ and $\sum_{x x}=\operatorname{cov}\left(\varepsilon_{x, t}\right)$ are the residuals covariance matrices of the full VAR model (1.2) and the restricted (without $\mathbf{Y}_{t}$ ) model (1.3) respectively.

This VAR model can be used where signals are brain networks time series. To apply GC, we use the multivariate Granger causality Matlab toolbox (Barnett and Seth, 2014). By taking nine simulated signals with known VAR coefficients from the demonstration script function of the aforementioned toolbox, on can calculate and visualised the GC between the signals. Figure 1.5 depicts this simulation. In this thesis, signals are the activity time series of fMRI-based restingstate networks (RSNs; examples in Figure 1.4). 

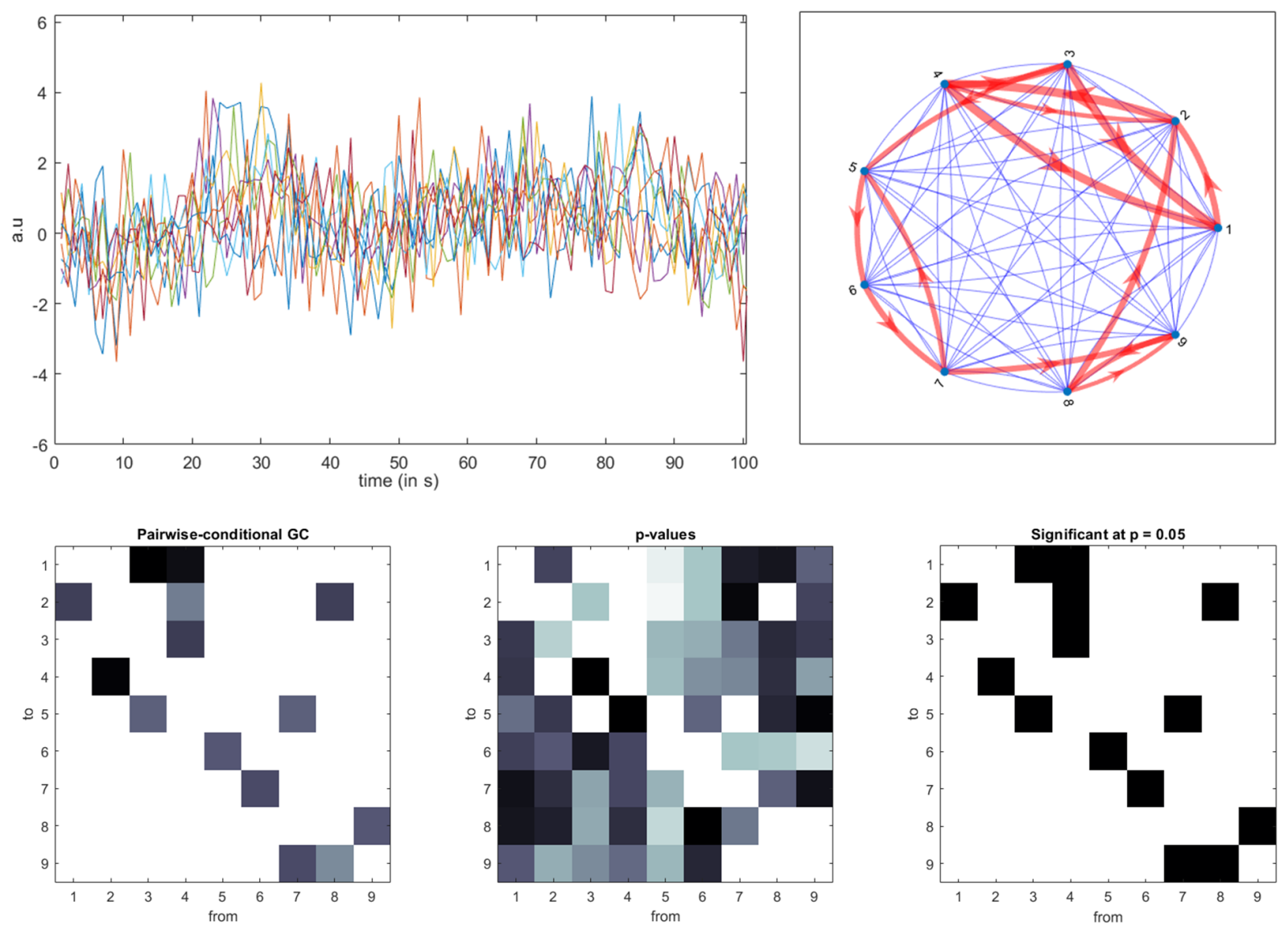

FIGURE 1.5: Granger causality (GC) between 9 simulated signals. Visualization of 9 signals with known VAR coefficients (top left); and the pairwise conditional GC, p-values, and significance matrices (bottom). These matrices can then be represented in a directed graph (top right; blue arrows are all possible connections; red arrows represent the significant causal connection). The thickness of the red arrows is proportional to the pairwise GC values.

\subsubsection{Phase coherence of brain network activity}

Analysis of phase-coherence between pairs of signals has been used for detecting patterns of co-activations in brain regions, especially in EEG studies. In fMRI, though, its application has been limited. Additionally, wavelet decomposition and wavelet transform coefficients represent a powerful tool to assess transients or non-stationary processes, and get insights in brain temporal dynamics. Cross-wavelet transform provides information of localised (in time and frequency) correlations between temporal signals (Torrence and Compo, 1998). It is also possible to derive from the wavelet transforms the phase shift between two signals. Combining these methods, we can extract wavelet-based coherence maps as it was originally performed in geophysics (Grinsted, Moore, and Jevrejeva, 2004). In neuroscience, the wavelet transforms have been applied, mainly as a filtering tool or a transient detector in EEG studies (Catarino et al., 2013; Hramov et al., 2015; Indic and Narayanan, 2011). Regarding resting-state fMRI, only few exploratory studies have been conducted in order to show non-stationarity in brain resting-state network fluctuations in healthy subjects using phase coherence analyses (Chang and Glover, 2010; Yaesoubi et al., 2015). 
The wavelet coherence between two signals $X$ and $Y$ is defined as

$$
R^{2}(t, s)=\frac{\left|\left\langle s^{-1} W^{X Y}(t, s)\right\rangle\right|^{2}}{\left\langle s^{-1}\left|W^{X}(t, s)\right|^{2}\right\rangle\left\langle s^{-1}\left|W^{Y}(t, s)\right|^{2}\right\rangle}
$$

where $W^{X Y}(t, s)=W^{X}(t, s) \cdot W^{Y}(t, s)^{*}$ is the cross-wavelet transform of signals $X$ and $Y$, and $\langle$. a smoothing operator (more details in Chang and Glover, 2010). $t$ and $s$ represent respectively the time and scale at which the formula is applied. Here, scales are wavelet periods in a $\log _{2}$-base, e.g. scale $1=2 \mathrm{~s}$ periods; scale $2=4 \mathrm{~s}$ periods, and so on). By using the information about the phasing between the signals, $\arg \left(W^{X Y}(t, s)\right)$, we can extract the wavelet coherence between pairs of signals. An example of such a map derived from two simulated signals is depicted in Figure 1.6. From the wavelet coherence maps (or periodograms) our dynamics metric of a 'time of coherence' is created, in the similar manner as the dwelling time in a brain state, as shown in Figure 1.3.c. More details about the time of coherence metric extracted from real rs-fMRI data are provided in Chapter 5.
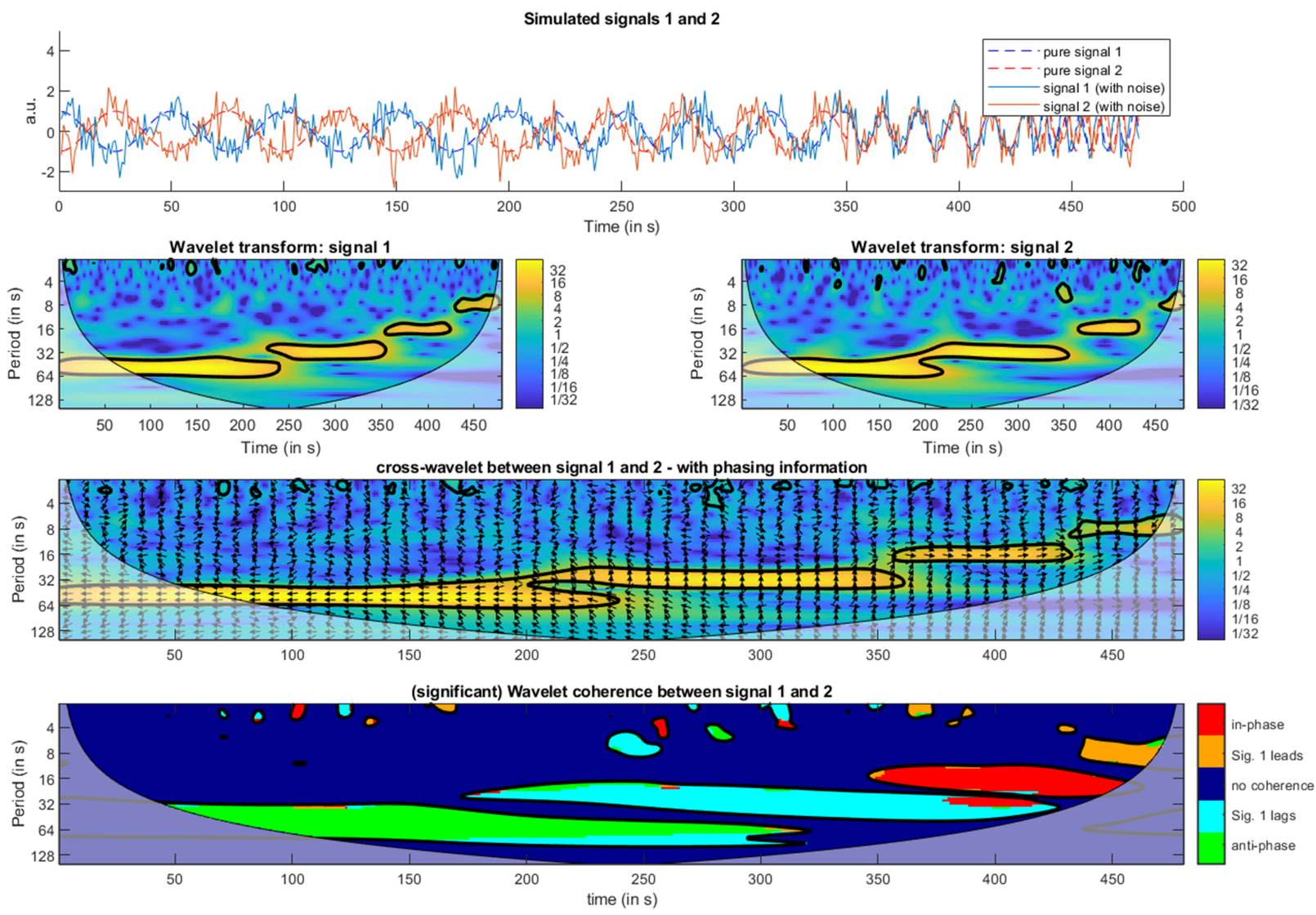

FIGURE 1.6: Wavelet coherence map between two simulated signals. Example of two noisy sinusoidal signals (top; signal 1 in blue, signal 2 in red), their respective wavelet transforms and the cross-wavelet transform (middle), and the wavelet-coherence maps of the signals (bottom). Arrows displays the phasing between the signals in the cross-wavelet periodogram. Contoured areas represent the significant in powers (middle), and in coherence (bottom). 


\subsubsection{Game theory as descriptor of network activity}

Humans have evolved as a species that can cooperate and compete with each other. This is the essence of the evolution of social animals, in order to survive. Since decision making to cooperate or compete is processed in the brain, the cerebrum should be the guarantor of our survival, and itself contain these propensities: defection and cooperation. This is usually translated through aggregation (cooperation) and segregation (competition) in brain connectivity. The evolutionary game theory can model the evolution in cooperating or defecting strategies, and their dynamics. These game dynamics in the brain are interesting to analyse. At the time of writing, only one paper presented its application upon rs-fMRI data, on a group of healthy subjects (Madeo et al., 2017). Madeo notably demonstrated the potential benefit of the evolutionary game theory on networks (EGN) model applied on rs-fMRI in lesioned brain (through simulation); and the potential of this predictive technique for further tailoring rehabilitation programs for individuals suffering from brain tumor, stroke or concussions, for instance. Madeo and his colleagues also applied this evolutionary game theory for forecasting epileptic seizure in an EEG study (Zingone, Mocenni, and Madeo, 2018). If and, are respectively the activity signals of networks and, the replicator dynamics used in the EGN model is defined as

$$
\dot{x}_{v}=x_{v}\left(1-x_{v}\right) \sum_{w=1}^{N} a_{v, w} \Delta p_{v, w}
$$

where $\sum_{w=1}^{N} a_{v, w} p_{v, w}$ determines the sign of the derivative $\dot{x}_{v}$, since $x_{v}\left(1-x_{v}\right)>0$; and because $p_{v, w}$, the payoff of the networks for being active or non-active, can, according to the model, only have two values $2 x_{w}-1$ or $1-2 x_{w}$ (whether $v$ tend to emulate $w$ or not; more detail in Madeo et al., 2017), we can simply rewrite the equation (1.6) as follows:

$$
\dot{x}_{v}=x_{v}\left(1-x_{v}\right) \sum_{w=1}^{N} a_{v, w}^{\prime}\left(2 x_{w}-1\right)
$$

where $a I_{v, w}$ is now a signed value, a weighted connectivity value or emulative power of $x_{v}$ towards $x_{w}$.

We can rewrite this differential equation (1.7) in matrix form, and by means of linear least square method one can estimated all the weights $\left(a_{v, w}^{\prime}\right)$, , i.e. the adjacency matrix entries, of the signed matrix $\mathbf{A}^{\prime}$. To have a better grasp of what it means for a network to have emulative propensities and how the networks game strategies and parameters or weights $\left(a_{v, w}^{\prime}\right)$ can help modeling fMRI neurodynamics, Figure 1.7 shows the possible dynamics between a pair of networks (Figure 1.7.a) and depicts the weights or adjacency matrix $\mathbf{A}^{\prime}$ when more than two networks are involved (Figure 1.7.b), We evaluate these weights that we coin Emulative Powers (EP) in Chapter 6 as a new dynamics metric and test its effectiveness in diagnosing neuropsychiatric disorders, such as ACA. 
a

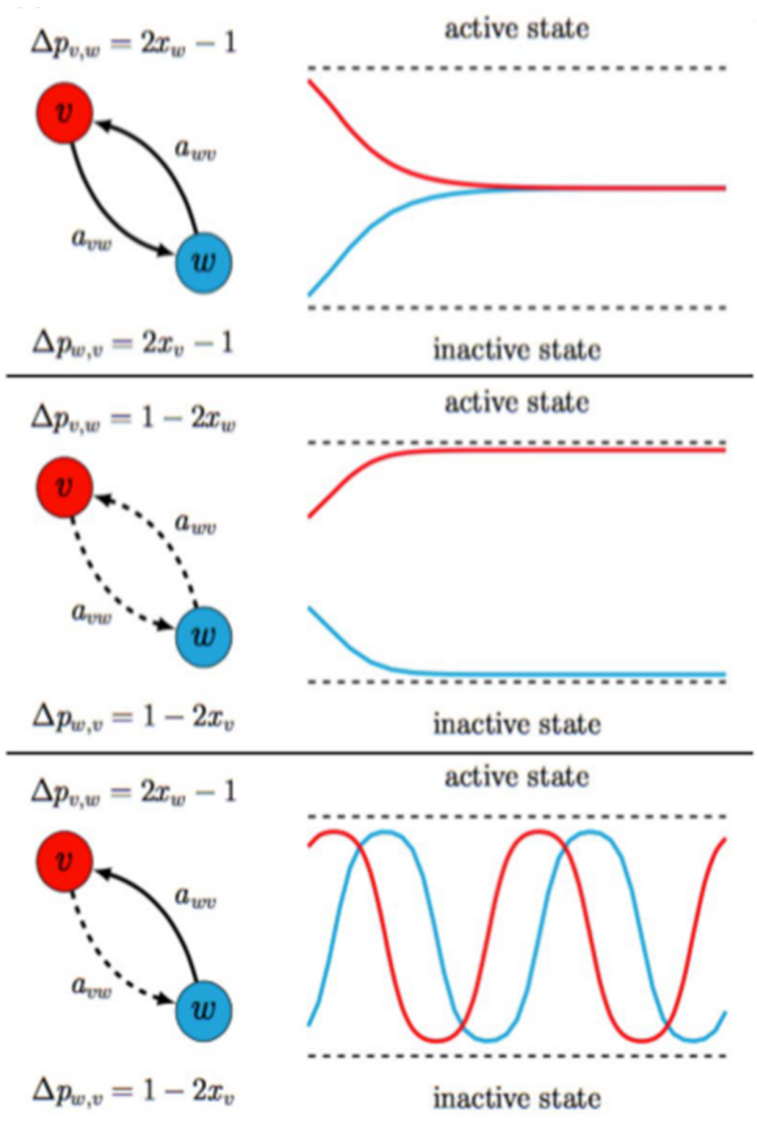

$\mathrm{b}$

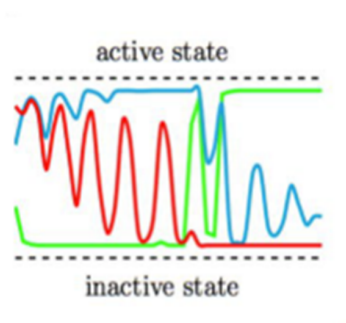

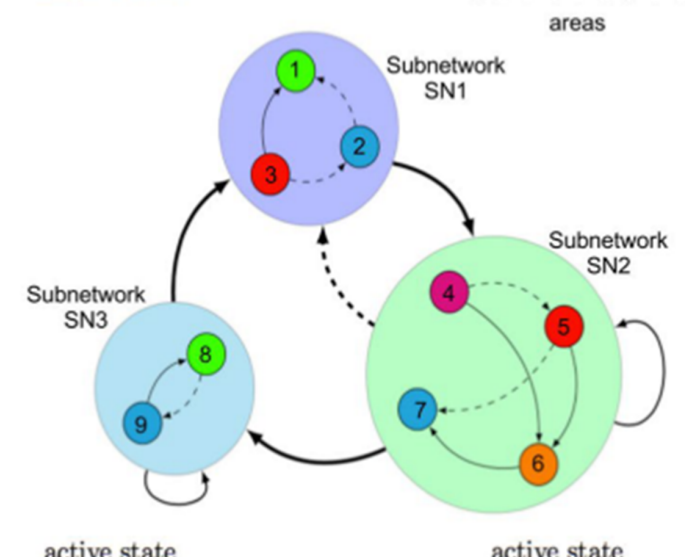

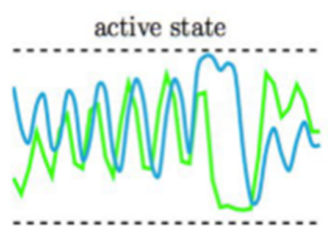

inactive state

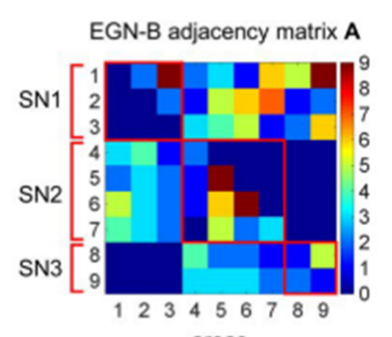

Subnetwork $\mathrm{SN} 1$

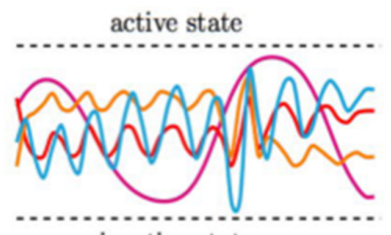

inactive state

FIGURE 1.7: Schematic description of the EGN model. (a) The three different configurations for a pair of networks $(v, w)$ and their dynamics, if their emulative power are both positive (top), both negative (nonemulative; middle), or opposite, i.e. one emulative, the other non-emulative (bottom). (b) A more complex configuration wherein nine networks are involved. Each subnetwork dynamics is also displayed, and the weights of their emulative connectivity is depicted in the A matrix on the top-right hand corner. Note that, here, the $\mathbf{A}$ matrix is not signed (i.e. $a_{v, w}^{\prime}>0$ ), hence show the absolute emulative power as in (1.6). Figure from Madeo et al., 2017.

\subsubsection{Thesis outline}

As described above, this thesis focuses on neuropsychiatric disorders, and their pathological brain dynamics. Neuropsychiatry is a vast field of research, thus, in order to narrow the research, we study two major neuropsychiatric disorders: the neurodevelopmental disorder for adolescents with autism spectrum disorder (ASD), and the accelerated cognitive ageing (ACA) in epilepsy.

The brain generates highly structured spatiotemporal patterns even in the absence of explicit task execution. Therefore, we focus our research on resting-state dynamic functional connectivity of the brain, namely its spontaneous neurodynamics. In this thesis, three methods are evaluated upon resting-state fMRI data: the Granger causality (measure of effective connectivity), the wavelet coherence (spectro-temporal analysis), and the evolutionary game theory approach on networks. These approaches are described in Chapter 1, Introduction.

In Chapter 2, functional connectivity between large-scale RSNs is measure in ACA and compared to aged- and education-level matched healthy elderly. 
Chapter 3 assesses Granger causality between large-scale networks, still in ACA patients, with discussions upon the RSNs involved and their pathological patterns of effective connectivity that are associated with the cognitive decline.

In Chapter 4, the effective connectivity between large-scale cognitive and limbic (emotion-related) RSNs is measured in ASD and compared to age- and IQ-matched healthy adolescents.

Chapter 5 describes first the implementation of the wavelet-coherence based approach between the participants' RSNs; compared the RSNs' pairwise times of coherence between the ASD and control group; and assess the classification power of the 'time of coherence' dynamic metric (or feature).

Chapter 6 implements the evolutionary game theory approach on networks activity time series in ACA and the (age- and education-matched) controls, tests classifiers, and concludes on the best image-based dynamics approach to discriminating ACA from healthy participants.

In Chapter 7, we assess the benefit of intensive music listening session on the dynamic connectivity of cognitive networks in healthy participants. Here, a new method of effective connectivity is tested: the Bayesian search; and it is compared to Granger causality.

Finally, Chapter 8 draws conclusions about neurodynamics in neuropsychiatric disorders, based on the results obtains in the precedent chapters and discusses the limitations but also the contributions and promises of the use of these new tools in psychoradiology of neuropsychiatric diseases. 

Chapter 2

Functional connectivity in Accelerated

Cognitive Ageing: an fMRI-evaluation of brain networks in cognitively deteriorated patients with epilepsy

[Submitted] 


\begin{abstract}
The goal of this study is to gain insight in the functional brain organisation of patients with epilepsy and Accelerated Cognitive Ageing (ACA).

The within and between brain network functional connectivity of ten epilepsy patients with ACA was identified using fMRI-ICA and then compared to 14 age- and education-matched healthy controls. Scan protocol consisted of two resting-state scans (RS1/RS2) and one task-based scan in between. RS1 provided the baseline functional architecture. RS2 revealed functional connectivity changes after cognitive effort. Functional connectivity was further measured in hierarchical correlation analyses and correlated to cognitive deterioration.

All well-known large-scale networks could be identified in both groups. At RS1, there was a reduced activation within the CEN in ACA. After cognitive effort, deactivation in the DMN and increased activation in the anti-correlated VAN was found in ACA. Hierarchical correlation analyses showed that in controls, there was a post-task enhanced temporal modulation of the SN from and towards the CEN and DAN. In ACA, this reciprocal modulation was significantly weaker. There was also a significantly weaker modulation between the DMN and VAN in ACA. There was an increase in post-task temporal connectivity between the cerebellum and both the DMN and CEN in ACA. Cognitive performance in controls was related to an efficient interaction within and between the attentional and executive networks, whereas in ACA, the emphasis was shifted to the connectivity between the DMN and the anti-correlated- and control networks.

ACA is characterised by a decreased efficiency in brain network interactivity and -flexibility. Switching between the DMN, SN and CEN appears less efficient and/or delayed in ACA, reducing the capacity of network modulations to changing external demands. This might be the echo of a loss of network segregation which is not so much interpreted as a pathological process but rather as an acceleration of the normal ageing processes.
\end{abstract}




\subsection{Introduction}

Cognitive deterioration has been a long-standing concern and important topic of interest in the field of adult epilepsy, especially in the context of ageing. Results from an increasing number of studies have indicated that the cognitive ageing trajectory in patients with epilepsy differs from the pattern observed in healthy ageing (Helmstaedter and Elger, 2009; Hermann et al., 2008; Hermann et al., 2006. In 1889, Gowers introduced the concept of 'epileptic dementia' (Rose, 2009). This type of cognitive deterioration is almost exclusively reported in childhood-onset chronic often refractory - epilepsies in which accumulation of seizure- and treatment-effects leads to slow and gradual cognitive decline, often over decades. The clinical outcome of this 'chronic accumulation model of deterioration' resembles the cognitive outcome in some forms of dementia (e.g. vascular dementia). In daily clinical practice clinicians are confronted with another form of cognitive deterioration that is less well described in the literature. In a subgroup of ageing adult patients with epilepsy a sudden inability to meet the demands of daily (working) life is observed. These patients do not fulfil the neuropsychological or clinical diagnostic criteria of a progressive neurological disease such as Alzheimer's disease. Since the majority of patients suffer from a late-onset epilepsy with low-frequency partial seizures, they do not fit into the aforementioned chronic accumulation model of deterioration either. In a recent review, we concluded that there may exist another pattern of cognitive deterioration in addition to the chronic accumulation model (Breuer et al., 2016b. In a subgroup of adult patients with epilepsy, cognitive deterioration may show a 'cascadic' rather than a progressive trajectory. Thus, deterioration takes place in a relatively short period of time, and not as a consequence of chronicity. Cognitive deterioration in this subgroup can be understood as an example of a 'second hit' phenomenon when seizures develop in an individual with reduced cognitive reserve capacity secondary to a previous 'first hit', such as traumatic brain injury or (cardio)vascular disease. 'Ageing' is a very important factor in this process since cognitive reserve capacity decreases with age and the ageing brain is less able to functionally compensate for additional interference, i.e. the pathogenic effects of first and second hits. The ageing population with an adult- or geriatric-onset epilepsy is at particular risk for this type of deterioration, taking into account that many factors converge in these late-onset epilepsies: comorbidity (especially stroke and other (cardio)vascular diseases), metabolic disturbances, increased inflammatory response to seizures, and the use of polypharmacy (Baram, 2012; Brodie, Elder, and Kwan, 2009; Leppik and Birnbaum, 2010; Palop and Mucke, 2009; Stefan et al., 2014; Trinka, 2003). We have proposed the descriptive term 'Accelerated Cognitive Ageing' (ACA) for this process.

In our previous work we described both the clinical (Breuer et al., 2016a), and neuropsychological characteristics and preliminary classification criteria of ACA (Breuer et al., 2019), in a series of patients with focal epilepsy and this hypothesised cascadic cognitive deterioration. The purpose of the current study was to study the functional network characteristics of ACA by comparing the MRI functional connectivity (FC) within and between brain networks in ACA-patients and matched healthy controls.

\subsection{Methods}

\subsubsection{Participants}

Fourteen adult outpatients with a confirmed diagnosis of epilepsy and ACA were recruited from the tertiary care centre for epilepsy Kempenhaeghe (Heeze, the Netherlands). Intellectual deterioration was defined as 1 standard deviation (SD) discrepancy between the actual Wechsler Adult 
Intelligence Scale-IV (WAIS-IV) Full Scale IQ (FSIQ) and the Oklahoma Premorbid Intelligence Estimate (OPIE-IV) estimated premorbid FSIQ. Exclusion criteria were mental retardation (FSIQ $<70$ before the age of 18), progressive epileptic syndromes, severe psychiatric disease at present state, and a history of epilepsy-related brain surgery. Patients with neurological or psychiatric diseases that could underlie such cognitive decline, (suspicion of) (neuro)degenerative diseases, progressive (neurological) disorders, or other causes of aforementioned cognitive deterioration were also excluded. The ACA-patients were consecutively age- and education-matched with 16 healthy controls with no history of neurological or psychiatric illness. A detailed medical history as well as demographic and clinical details were obtained. This study was approved by the National Medical Ethical Committee. All subjects signed informed consent and gave permission to use the obtained clinical data for scientific purposes.

After checking and pre-processing the fMRI-data (see section Resting State Network (RSN) extraction), four ACA-patients and two healthy controls were excluded due to excessive head motion $(n=3)$, MRI artefacts $(n=2)$ and an unexpected detection of a brain tumour $(n=1)$. The effective sample therefore consisted of 10 ACA-patients and 14 healthy controls. Main demographic and clinical data are shown in Table 2.1

\subsubsection{Cognitive measures}

Intelligence was measured using the Dutch version of the WAIS-IV (Wechsler, 2008). For an estimation of the premorbid intelligence, the OPIE-IV formula was applied. The OPIE-IV equations are derived from hierarchical regression models using raw scores of two WAIS-IV subtests (Vocabulary (V) and Matrix Reasoning (MR)), age, education, sex and ethnicity (Holdnack et al., 2013). OPIE-IV predictions were calculated for the FSIQ, verbal intelligence (Verbal Comprehension Index (VCI)) and performance abilities (Perceptual Reasoning Index (PRI)), resulting in OPIEIV_FSIQ, OPIE-IV_VCI and OPIE-IV_PRI scores, respectively. There was a significant (strong) correlation between the WAIS-IV FSIQ and the OPIE-IV_FSIQ in the healthy controls $(\mathrm{r}=.80, \mathrm{p}=<$ .001). Similar effects were found for VCI and OPIE-IV_VCI $(\mathrm{r}=.89, \mathrm{p}=<.001)$ and PRI and OPIEIV_PRI $(\mathrm{r}=.79, \mathrm{p}=<.001)$, comparable to findings of Holdnack et al. (2013) in the normal population (Holdnack et al., 2013). Deterioration scores were calculated by subtracting the estimated premorbid IQs (OPIE-IV_scores) from the actual obtained IQs, resulting in three IQ-deterioration scores: DET_FSIQ, DET_VCI and DET_PRI. The Dutch translation of the Wechsler Memory Scale Fourth Edition (WMS-IV16) was used for assessment of memory functions. Neuropsychological data in mean (M) and standard deviation (SD) are shown in Table 1.

\subsubsection{MRI protocol}

Anatomical images (including a T1-weighted 3D Fast Field Echo (FFE) sequence) were acquired using a 3.0 T imaging system (Philips Achieva) with echo-time (TE) $=3.8 \mathrm{~ms}$, Repetition time (TR) $=8.4 \mathrm{~ms}$; flip-angle $=8^{\circ}$; field of view $(\mathrm{FOV})=240 \times 240 \mathrm{~mm} 2$, with 180 sagittal slices; and voxel size $=1 \mathrm{~mm}^{3}$; with SENSE factor of 1.5 , and scan length of $9 \mathrm{~min}$.

All subjects underwent a series of functional runs, consisting of two resting-state (RS) fMRI-scans and one task-based scan with an activation paradigm in between (silent word-generation). The first resting-state fMRI-scan (RS1) provided the baseline functional architecture in both subject groups. The following task-based fMRI-scan solely served as a means to initiate cognitive (taskbased) activity. Data of this scan were not further analysed. The second resting-state scan (RS2) revealed whether functional connectivity changed after cognitive effort. Functional MRI-data were acquired using multi-echo echo-planar imaging (ME-EPI) sequences with 3 echoes. Parameters of 
TABLE 2.1: Demographic, clinical and neuropsychological characteristics of the ACA and control subjects

\begin{tabular}{|c|c|c|}
\hline & ACA & Healthy controls \\
\hline Age in years $-M(s t d)$ [range] & $61.3(8.9)$ [50-74] & $62.2(9.8)[47-79]$ \\
\hline Gender & $70.0 \%$ male & $35.7 \%$ male \\
\hline Handedness & $100.0 \%$ right-handed & $92.9 \%$ right-handed \\
\hline Age at epilepsy onset & $35.0(14.6)$ [15-59] & - \\
\hline Duration of epilepsy & $22.3(15.2)[1-50]$ & \\
\hline Type of epilepsy & $\begin{array}{l}40.0 \% \text { cryptogenic localized } \\
40 \% \text { sympomatic } \\
20 \% \text { idopathic }\end{array}$ & - \\
\hline Dominant seizure type ${ }^{a}$ & $\begin{array}{l}10.0 \% \text { simple partial } \\
20.0 \% \text { complex partial } \\
20 \% \text { absence } \\
50 \% \text { seizure free }\end{array}$ & - \\
\hline Status epilepticus & $50.0 \%$ yes & - \\
\hline Seizure frequency & $\begin{array}{l}50.0 \% \text { seizure }(\mathrm{sz}) \text { free } \\
0.0 \%<1 \mathrm{sz} / \mathrm{y} \\
20.0 \% 1-5 \mathrm{sz} / \mathrm{y} \\
20.0 \% 1 \mathrm{sz} \text { per } 2 \text { months } \\
0.0 \% \text { montly sz } \\
10.0 \% \text { weekly sz } \\
0.0 \% \text { daily sz }\end{array}$ & - \\
\hline Drug load ${ }^{\mathrm{b}}$ & $1.0(0.4)$ & - \\
\hline \multicolumn{3}{|l|}{ WAIS-IV indexes } \\
\hline FSIQ & $76.7(8.7)^{*}$ & $108.4(13.4)$ \\
\hline VCI & $94.7(10.6)$ & $106.2(12.2)$ \\
\hline PRI & $75.8(6.9)^{*}$ & $103.2(13.8)$ \\
\hline WMI & $79.6(10.0)^{*}$ & $104.9(13.3)$ \\
\hline PSI & $67.0(14.4)^{*}$ & $114.6(8.7)$ \\
\hline \multicolumn{3}{|l|}{ OPIE-IV scores } \\
\hline OPIE-IV FSIQ & $98.8(9.2)$ & $109.6(12.4)$ \\
\hline OPIE-IV VCI & $95.2(11.7)$ & $105.6(14.6)$ \\
\hline OPIE-IV PRI & $96.8(9.3)$ & $102.5(12.4)$ \\
\hline \multicolumn{3}{|l|}{ Deterioration scores } \\
\hline DET FSIQ & $-22.1(5.0)^{*}$ & $1.3(8.2)$ \\
\hline DET VCI & $-0.5(8.9)$ & $0.2(6.0)$ \\
\hline DET PRI & $-21.0(4.5)^{*}$ & $0.0(8.7)$ \\
\hline \multicolumn{3}{|l|}{ Memory scores } \\
\hline Auditory & $93.9(9.7)^{*}$ & $109.9(12.6)$ \\
\hline Visual & $92.7(7.0)$ & $102.8(10.9)$ \\
\hline Delayed memory & $93.5(8.4)^{*}$ & $106.3(10.9)$ \\
\hline
\end{tabular}

${ }^{*} \mathrm{p}<.01$ significant difference between groups. WMI: Working Memoey Index. PSI" Processing Speed Index.

a Dominant seizure type is determined for the two years preceding neuropsychological assessment

$\mathrm{b}$ The prescribed daily dose of antiepileptic medication divided by the define daily dose (Lammers et al., 1995). 
these scans were: $\mathrm{TEs}=12,35,58 \mathrm{~ms}, \mathrm{TR}=2 \mathrm{~s} ; \mathrm{FA}=90^{\circ}$; SENSE factor $=2.7 ; 208$ dynamics for a total of 7 minutes; 27 axial slices (with no gap), $64^{*} 64$ matrix FOV, with a $3.5 \times 3.5 \times 4.5 \mathrm{~mm} 3$ voxel size.

\subsubsection{Multi-echo fMRI data pre-processing and resting-state network extraction}

As mentioned, fMRI resting-state data were obtained using Multi-Echo EPI sequences which have been proven to significantly de-noise data and improve (pre)processing. By using an ICAdecomposition, an optimal combination of 3 echoes using relaxation maps R2*, and a least squares approach for regression analysis, BOLD (activity-related) and non-BOLD (noise) signal components in resting state data can be cleanly separated and substantially improve the power of multisubject fMRI studies (Dipasquale et al., 2017; Kundu et al., 2012, allowing to have similar power (sensitivity) of single-echo fMRI studies with half a sample size (Lombardo et al., 2016).

A modified version of the python script 'meica.py' ${ }^{1}$ was used for data pre-processing. As a result, cleaned fMRI data as well as an overview of the identified BOLD RSNs for each subject per scan were obtained. After data cleaning, group RSNs were defined using group-level ICA with MELODIC v.3.14 (Beckmann and Smith, 2004) from FMRIB's Software Library (FSL ${ }^{2}$ ). For this group-level ICA, the same processing steps as in our previous study were used (Bernas et al., 2018), changing only the Gaussian kernel for spatial smoothing to $5.0 \mathrm{~mm}$ full width at half maximum, and the dimensions for the projection to 18 independent components. This dimensionality was chosen by averaging the number of 'good' (BOLD-related) components per subject/session obtained during de-noising steps for the multi-echo data.

Using the aforementioned group RSN-maps and applying dual regression technique (Beckmann et al., 2009; Filippini et al., 2009) on the cleaned RS-fMRI, the subject- and session-specific RSNmaps and their associated time series were extracted. From these subject- and session-specific RSN-maps, we conducted spatial statistical analysis within each network, and between-network (correlation) connectivity analysis. This analysis is explained in detail below.

\subsubsection{RSN spatial analysis}

\section{ACA vs. healthy controls}

First, a comparison was made between subject groups for both RS1 and RS2 (independently), using a non-parametric (using 1000 label permutations) t-test (Winkler et al., 2014). Obtained tscores were threshold-free cluster enhanced (TFCE) to ensure a good trade of between arbitrary cluster thresholding and a conservative voxel-wise thresholding (Smith and Nichols, 2009). Pvalues were family-wise error rate corrected for multiple comparison (set at .05). Four contrast maps could be assessed in terms of decrease or increase in brain network activation between groups: RS1 ACA > RS1 controls; RS1 ACA < RS1 controls; RS2 ACA > RS2 controls; RS2 ACA < RS2 controls.

\section{RS1 vs. RS2}

Secondly, a within-group comparison was made between RS1 and RS2 for both ACA-patients and healthy controls in order to evaluate whether functional connectivity had changed after a cognitively demanding task compared to baseline connectivity. A non-parametric (1000 flip-sign permutation) one-sample t-test was applied upon the subject-specific difference maps (RS2 minus

\footnotetext{
${ }^{1}$ https://bitbucket.org/prantikk/me-ica

2 www.fmrib.oc.ac.uk/fsl
} 
RS1). This is the equivalent of a randomised two-sample paired t-test, using 1000 controlledpermutation (hence, RS1 and RS2 of a subject cannot be in the same group). Again, four contrast maps could be derived from this analysis: RS2 controls > RS1 controls; RS2 controls < RS1 controls; RS2 ACA > RS1 ACA; RS2 ACA < RS1 ACA.

\subsubsection{RSN correlation and hierarchy}

Leading on from the above mentioned spatial contrast analyses, a hierarchical correlation matrix was plotted to assess whether differences in functional connectivity between ACA and healthy controls originate from a difference in the modulation of connectivity between resting-state networks, i.e. whether functional connectivity between the RSNs in time was different in the subject groups. Using the time series associated with each subject- and session-specific RSNs, the average correlation distances between each pair of networks could be assessed. Then, hierarchically correlated clusters of networks were plotted. Using two-sample t-tests to compare groups and paired t-tests to compare sessions, the significant pairs of RSNs showing changes in functional connectivity (z-scores of correlation coefficients) between groups or between sessions were depicted.

\subsubsection{RSN functional connectivity and cognitive measures}

Finally, the correlation between functional connectivity (correlation z-scores) and the FSIQ, PRI, VCI and PSI was assessed. The functional connectivity matrices for each subject in RS1 and RS2 were extracted and correlated with the subject's aforementioned neuropsychological scores using Pearson correlation coefficients.

\subsection{Results}

\subsubsection{Resting-state network extraction}

Using the multi-echo data cleaning followed by a group-independent component analysis, 18 brain networks could be extracted. Figure 2.1 shows these RSNs (or independent components (ICs)) extracted from the pre-processed datasets of all 24 subjects and their two resting-state fMRI scan sessions. Most well-known large-scale networks described in literature could be retrieved (Cole, 2010; Damoiseaux et al., 2006; Smith et al., 2009a: the visual network system (medial, lateral and occipital visual cortices; MED-VIS, LAT-VIS, and OCC-VIS respectively), the auditory system (AUD), the sensorimotor network system (MED-SENS, and LAT-SENS), the default mode network (DMN), the dorsal and ventral attention networks (DAN and VAN, respectively), the central executive network (CEN; consisting of the fronto-parietal left and right cortex (FPL and FPR) and the cingulate gyrus (CING)), the salience network (SN) and cerebellum (CB). Four networks were considered noise or artefactual networks and were discarded from further analysis as they showed high-frequency dominant power, and/or did not comprise grey/cortical matter. The extracted group RSNs are shown in Figure 2.1.

\subsubsection{RSN spatial connectivity analysis}

ACA vs. healthy controls

Four contrast maps could be assessed in terms of decrease or increase in brain network activation 


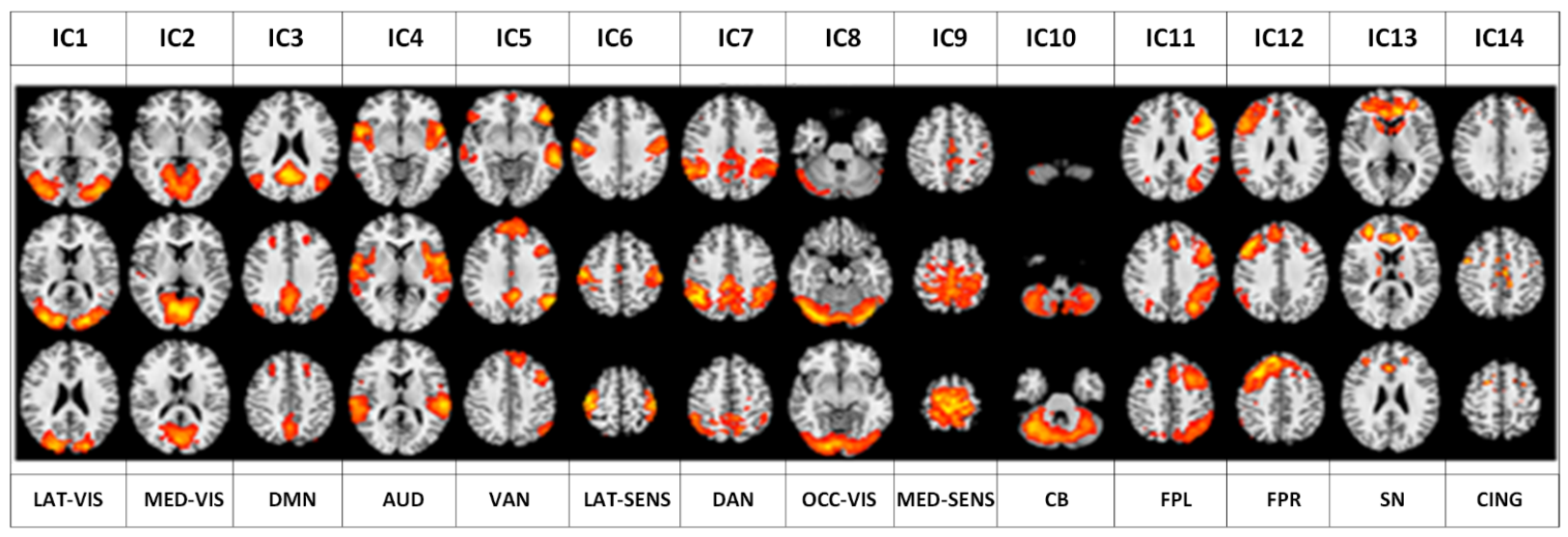

FIGURE 2.1: Resting-state networks extracted from group-ICA.

between groups: RS1 ACA > RS 1 controls; RS1 ACA < RS 1 controls; RS2 ACA > RS2 controls; RS2 ACA < RS2 controls. Figure 2.2 presents these results.

\section{RS1 vs. RS2}

The within-group comparison on functional connectivity revealed that in the ACA-group, besides less activation in the visual and sensorimotor networks, also a decrease in activation in the CEN (both the FPL- and FPR-network) and DMN was seen in RS2 compared to RS1. An increase in activation in RS2 was seen in the VAN. In the healthy controls, there was a decrease in RS2-activation in the FPL-network, cingulate gyrus (both CEN) and the SN, besides decrease in sensory brain networks (auditory and visual networks). In controls, no increased activation in RS2 compared to RS1 was found.

\subsubsection{RSN correlation and hierarchy}

Figure 2.3 shows the hierarchically correlated clusters of networks in ACA and the healthy controls in both RS1 and RS2 (right) and significant differences in temporal functional connectivity between both groups regarding the attention-, control- and executive- networks (left).

In both subject groups, there was a common high temporal FC between the visual networks and the sensorimotor system (A-cluster in Figure 2.3). This visuo-sensorimotor cluster was nevertheless more correlated with the DMN in healthy controls. In both groups, there was a high temporal FC between the CEN and the DAN (B-cluster). In addition, temporal anti-correlations between the SN, cerebellum and visual- and sensorimotor networks (C-cluster) were found in both groups.

However, significant groups differences in pairwise network functional connectivity were also present, and are depicted in Figure 2.3 (D to I). These differences involved mainly the DMN, DAN, VAN, SN and CING networks, i.e. the cognitive-related networks.

\subsubsection{RSN functional connectivity and cognitive measures}

The correlation within and between functional network connectivity and the FSIQ, PRI, VCI and PSI is shown in figure 2.4. Correlations between cognitive measures and the visual network system, the auditory system and/or the sensorimotor network system are not shown. 


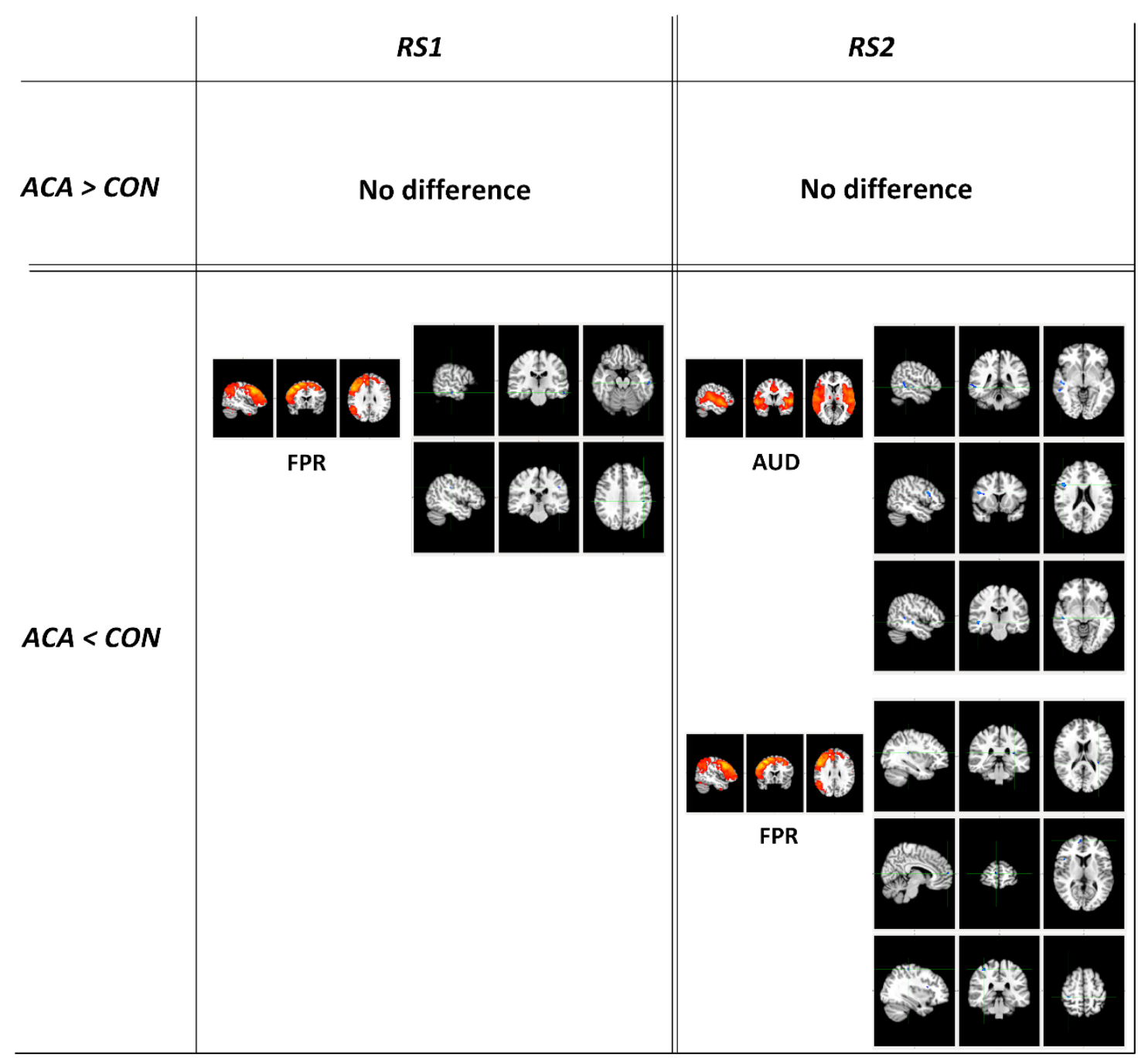

FIGURE 2.2: Most relevant within-network differences between the groups for RS1 (on the left) and RS2 (on the right). 

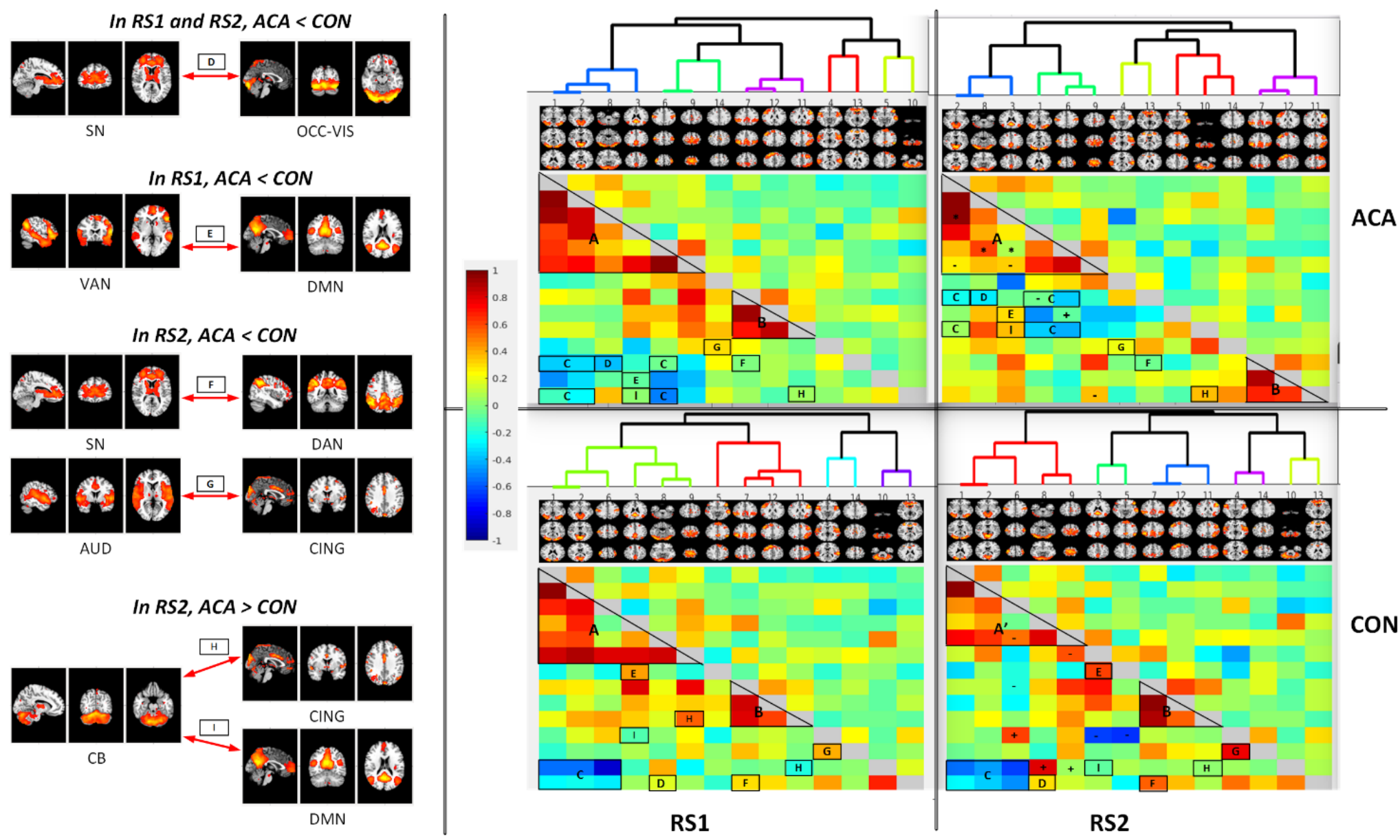

RS1

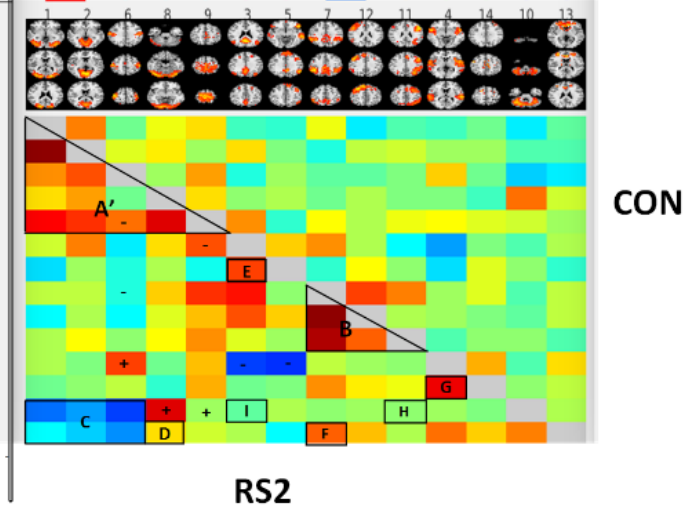

FIGURE 2.3: Network connectivity differences between ACA and Controls. Dissimilarity dendrograms (based on averaged full correlation) for each subject group and each scan (right figure); and pairs of RSNs showing significant FC differences (left figure). A-cluster: LAT-VIS, MED-VIS, OCC-VIS, LAT-SENS, MED-SENS and DMN. B-cluster: FPL, FPR and DAN. C-cluster: LAT-VIS, MED-VIS, LATSENS, CB and SN. A'-cluster: similar to A-cluster with a DMN cluster dissociation in RS2. D to I: pairs of RSNs of which the FC differed significantly between groups (ACA vs Controls). D: SN $\leftrightarrow$ OCC-VIS sign. weaker in ACA in RS1 and RS2. E: DMN $\leftrightarrow$ VAN sign. weaker in ACA in RS1. F: DAN $\leftrightarrow$ SN sign. weaker in ACA in RS2. G: CING $\leftrightarrow$ AUD sign. weaker in ACA in RS2. H I: CB $\leftrightarrow$ CING and DMN sign. stronger in ACA in RS2. The network hierarchy tend to change more in ACA than in the healthy controls. 


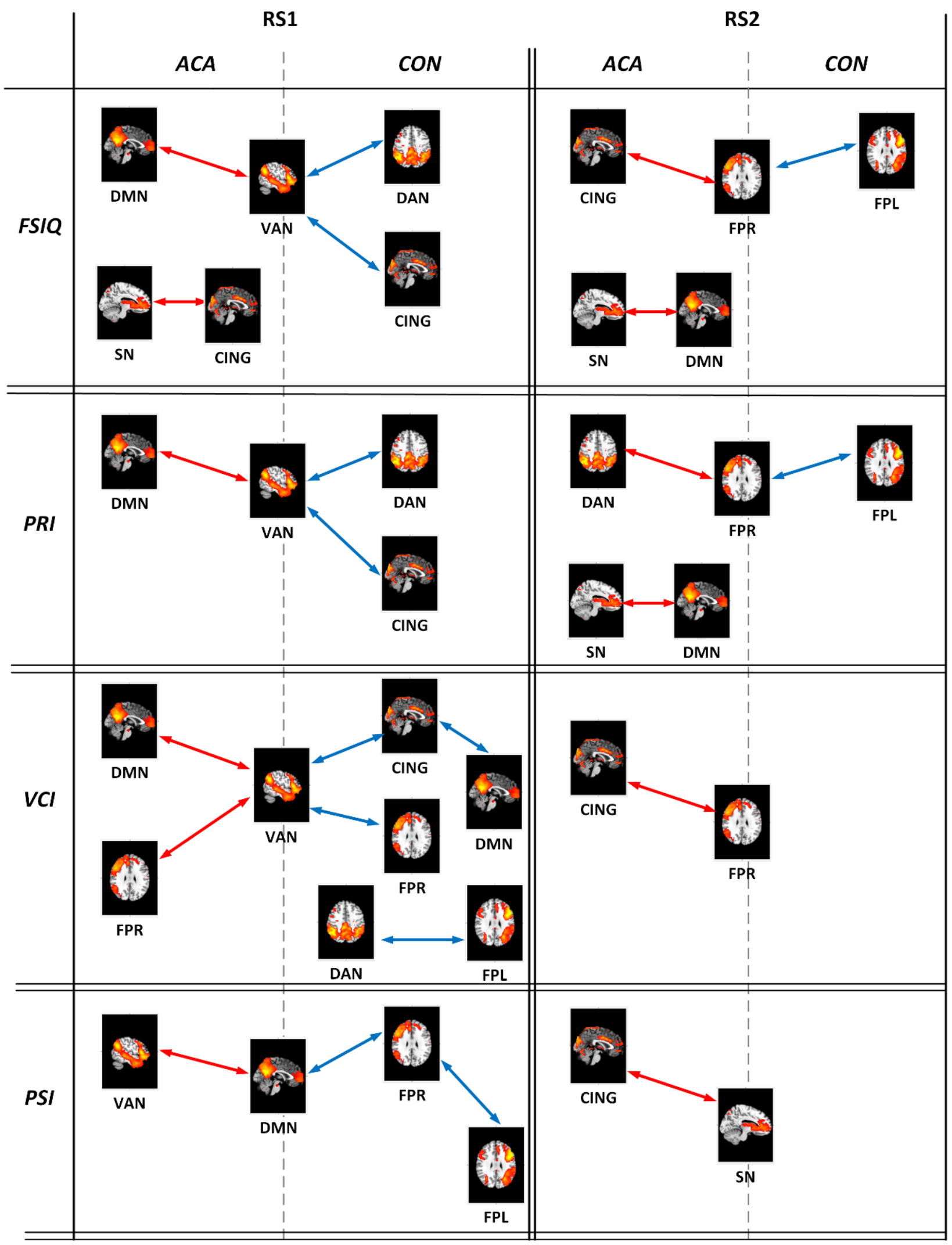

FIGURE 2.4: Significant correlations between cognitive measures and the between-network functional connectivity and in ACA (red) and healthy controls (blue). 


\subsection{Discussion}

In previous studies we described both clinical and neuropsychological characteristics and preliminary classification criteria of Accelerated Cognitive Ageing as a specific form of deterioration in (especially adult-onset) epilepsy. The purpose of the current study was to gain insight in the brain organisation of patients with ACA by comparing the MRI functional connectivity within and between brain networks in ACA-patients and healthy controls. In our age- and education matched study population of ACA-patients and healthy controls, the fMRI analyses showed the following results:

Functional brain architecture is not different in ACA patients compared to matched controls, i.e. all well-known large-scale networks could be identified in both subject groups. This shows that the clinical outcome of ACA (i.e. cascadic cognitive deterioration) is not based on changes in the basic functional network design.

At baseline resting-state, there proved to be a reduced activation within the CEN in ACA-patients relative to healthy controls. The fronto-parietal CEN is, together with the fronto-insular $\mathrm{SN}$ and the medial prefrontal-medial parietal DMN, part of the well-known trinity of large-scale networks that are theorised to be involved in a dynamic interplay to regulate information processing and cognitive control in the pursuit of a higher behavioural goal. Studies indicate that there is a causal neural mechanism by which the CEN and SN regulate activity in the DMN in order to mediate transitions between rest and task-engaged states to optimise cognitive performance (Chen et al., 2013). Reduced functional activity within these networks has been suggested to be one of the main causes of cognitive decline in ageing adults (Ferreira and Busatto, 2013; Marstaller et al., 2015; Onoda, Ishihara, and Yamaguchi, 2012b) and has been associated with cognitive dysfunction in numerous neurological diseases such as traumatic brain injury and Alzheimer's disease (Hafkemeijer, Grond, and Rombouts, 2012). Whether this reduced intra-network connectivity as seen in the ACA-group should be interpreted as a dysfunction within the network itself or whether it reflects lower flexibility of network interactivity (Avelar-Pereira et al., 2017) is still debated, but our results suggest the latter.

After cognitive effort, differences in functional connectivity became apparent between groups. In the healthy controls, parts of the CEN as well as the SN became less activated in rest after cognitive strain compared to baseline resting-state. These task-induced deactivations in these cognitive control systems are generally known to reflect a switch away from the constrained processing during the task to the unconstrained default mode processing in passive condition34. Following this, there was also a decrease in activity in the CEN in the ACA-patients. However, there appeared to be a post-task deactivation in ACA-patients in the DMN as well. At the same time, an increase in activity was found in the anti-correlated VAN, whereas no increase in activation was seen in any of the RS2-networks in the healthy controls. This post-task activation of the VAN and simultaneous deactivation of the DMN in ACA seems counter-intuitive, taken into account that the post-task condition is a (return to) the passive condition rather than a cognitively demanding condition that requires activation of so-called task-positive networks such as the VAN. Studies on age differences in cognitive control report similar processes which are interpreted as reflecting age-related slowing and a decreasing ability to reallocate resources away from default-mode processing and towards the task. Consequently, additional networks such as the VAN are recruited to compensate for lingering default-mode processes during a task in order to retain cognitive efficiency (Persson et al., 2007 . Other studies confirmed that there is an age-related reduction in the ability to suspend default mode activity during task performance in healthy adults (Grady et al., 2006; Lustig et al., 2003 as well as (pathologically) in patients with dementia (Greicius et al., 2004). This suggests that this lingering of activity in default-mode regions during task performance reflects a vulnerability of the brain in both normal and pathological (biological as well as cognitive) 
ageing processes. In other words: there might be functional connectivity deficiencies in interaction and switching between the DMN and the attention and executive networks in ACA-patients similar to the decreasing flexibility of network interaction in normal ageing and neurological disease, but at a younger age than their healthy peers.

The hierarchical correlation analyses showed that in healthy controls, the temporal reciprocal modulation between the DMN, SN and the attention networks is different at baseline resting state compared to rest after a cognitive task. In RS2, there was an enhanced modulation of the SN from and towards parts of the CEN and DAN, which is in accordance with the idea that through the $\mathrm{SN}$ other networks are (de)activated. In the ACA patients, this reciprocal modulation was significantly weaker. Furthermore, there was an increased reciprocal modulation between the DMN and anti-correlated VAN in both subject groups, though with a significantly weaker modulation (i.e. weaker anti-correlation) in ACA. Again, this suggests that the switching between the DMN and other networks is less efficient and/or delayed in ACA. Remarkably, there was an increase in posttask temporal functional connectivity between the cerebellum and both the DMN and parts of the CEN in ACA. Such increased functional connectivity is commonly interpreted as compensatory reallocation or dedifferentiation (Guo et al., 2015).

From the final analyses on functional connectivity in relation to cognitive performance, the connectivity between the task-negative DMN and the task-positive VAN at baseline resting-state proved to be highly correlated with all measures of intellectual performance in ACA. This is remarkably different from the pattern seen in healthy controls, in whom better cognitive performance was mainly associated with enhanced functional connectivity between the task-positive dorsal and ventral attentional networks and the CEN. A comparable phenomenon was observed in RS2 where in ACA-patients, the functional connectivity between the SN and the DMN was associated with better cognitive performance, whereas enhanced connectivity within the CEN was correlated with cognitive function in healthy controls. In other words: in healthy controls, enhanced cognitive performance seems to be related to an efficient interaction within and between the attentional and executive networks which are indeed known to show increased simultaneous activation during externalised attention-demanding cognitive tasks, whereas in ACA-patients, the emphasis has been shifted to the connectivity between the DMN and the anti-correlated- and control networks in the preservation of a certain cognitive performance level. This again, might be a reflection of decreased efficiency in network interactivity, namely in the primarily control and switching between networks. One could hypothesise that this is the echo of loss of network segregation which is described as dedifferentiation and lesser anti-correlation connectivity between opposed brain networks. The rate of loss of functional segregation between the DMN and the CEN, $\mathrm{SN}$ and attentional networks has been associated with ageing-related cognitive decline, starting with a decline in the information processing speed and slow decrease in performance abilities (Ng et al., 2016). Moreover, the functional segregation between the DMN and CEN, and between the DMN and the attentional networks might be predictive of the amount of cognitive reserve as stated by Franzmeier and colleagues (Franzmeier et al., 2017). This seems to apply to ACApatients who seem to have had a comparable level of intelligence as their healthy peers before onset of disease, but who appeared to be significantly cognitively deteriorated due to diminished cognitive reserve capacity, with most impairment seen in the information processing speed and performance abilities. However, from longitudinal studies it is known that the functional segregation between the DMN and the other networks (i.e. the CEN) shows an initial increase with ascending age before showing a decrease starting at the age 65-70 ( $\mathrm{Ng}$ et al., 2016), which is several years later than might be the case in ACA-patients (mean age 61.3 in our sample and 4/10 subjects 65 years old). The same seems to apply to the functional segregation between the DMN and other aforementioned networks (Spreng et al., 2016; Tsvetanov et al., 2016). 


\subsection{Conclusion}

In conclusion, Accelerated Cognitive Ageing on brain network level is characterised not by differences in the functional architecture, but by differences in brain network interactivity and -flexibility, hence not in the brain topology but in brain dynamics. Switching between the DMN, SN and CEN appears less efficient and/or delayed in ACA-patients, reducing the capacity of network modulation to changing (external) demands. This may underlie the cognitive deterioration, despite of what appear to be compensatory mechanisms to (re-)engage cognitive control in the form of recruitment of additional task-positive networks or by holding on to the segregation between the DMN and control networks during cognitive performance. This is not so much interpreted as a pathological process but rather as an acceleration of normal ageing processes due to diminished reserve capacity. Therefore, the basic mechanism underlying the deterioration in patients with epilepsy and Accelerated Cognitive Ageing may not be a pathological mechanism, but rather a characteristic of an ageing brain, though at a much younger age. This phenomenon is therefore correctly termed 'Accelerated Cognitive Ageing' also from the perspective of brain network organisation. 
Chapter 3

\title{
Accelerated Cognitive Ageing in epilepsy: exploring the effective connectivity between resting-state networks and its relation to cognitive decline
}

\author{
[Published in Cell press: Heliyon]
}




\begin{abstract}
This study aims at understanding the dynamic functional brain organisation in Accelerated Cognitive Ageing (ACA) in epilepsy. We also assess to which extend the (abnormal) effective connectivity between brain networks correlates with the (estimated) decline in IQ scores observed in the ACA patients.

Two multi-echo resting-state fMRI scans of 10 ACA patients and 14 age- and education-matched healthy controls were acquired. A task-based fMRI was acquired in-between those two scans, for possible cognitive fatigue effects on reserve capacity. Granger causality (GC), a measure of effective connectivity between brain regions, was applied on 7 major cognitive networks, and group-wise compared, using permutation testing statistics. This was done on each of the restingstate sessions independently. We assessed the correlation between the cognitive deterioration scores (representing cognitive decline), and the paired-networks granger causality values.
\end{abstract}

The cingulate cortex appeared to be more engaged in ACA patients. Its dynamics towards the right fronto-parietal cortex, salience network, and the dorsal attention networks (DAN) was stronger than in controls, only in the first resting-state scans. The Granger causality from the DAN to the default mode network (DMN) and from the ventral attention network (VAN) to the left fronto-parietal network (FPL) was also stronger in ACA patients and again only in the first scan. In the second resting-state scan, only the DMN was more strongly connected with the cingulate cortex in ACA patients. A weaker GC from DMN to FPL, and stronger GC from the salience network to cingulate cortex were associated with more decline in the Full-scale IQ and more GC from DMN to VAN would lead to more decline in the Perceptual Reasoning Index in ACA.

The results are in line with the hypothesis of over-recruitment at low cognitive load, and exhaustion at higher cognitive load, as shown by the $\mathrm{CRUNCH}^{1}$ model for ageing. Moreover, the DMN to VAN directed connectivity strongly correlates with the (estimated) decline in the Perceptual Reasoning Index, which is also in line with a recent study on ageing with mild cognitive impairment in elderly, and the PASA ${ }^{2}$ model. This study therefore supports the idea that the cognitive decline in our patients resembles the decline observed in healthy ageing, but in an accelerated mode. This study also sheds light on the directions of the impaired connectivity between the main networks involved in the deterioration process, which can be helpful for future development of treatments solutions.

${ }^{1}$ CRUNCH: Compensation-Related Utilization of Neural Circuits Hypothesis

${ }^{2}$ PASA: Posterior-Anterior Shift in Aging 


\subsection{Introduction}

Cognitive impairment is a common comorbidity in patients with epilepsy. It has been estimated that up to $65 \%$ of all patients with epilepsy show cognitive impairment, which also account for about half the burden of disease (Helmstaedter and Elger, 2009; Helmstaedter et al., 2014). Such impairments have frequently been reported in literature but only for specific cognitive functions, for specific types of epilepsy, e.g., memory impairments in temporal lobe epilepsy, or executive function impairment in frontal lobe epilepsy. Cognitive decline (i.e. global cognitive deterioration) is less frequently reported (Baram, 2012; Breuer et al., 2016b; Hermann et al., 2008; Hermann et al., 2006). In addition, more global cognitive decline had been already identified with the induction of the concept of epilepsy dementia by Growers in 1889 (Rose, 2009). Research on this epilepsy comorbidity suggests that it almost exclusively occurs in case of child on-set epilepsy where accumulation of medication and (tonic-clonic) seizures over decades yield to gradual decline of higher cognitive functions. Therefore, the decline is viewed as a 'chronic accumulation' model, where the cognitive outcomes of the patients resembles those in dementia. However, recently we proposed a new 'cascadic' model of cognitive decline in epilepsy (Breuer et al., 2016b), where patients display cognitive deterioration but do not meet the criteria of Alzheimer Disease or the aforementioned epilepsy dementia comorbidity. The process is seen in adult-onset epilepsy and seem to mimic 'healthy' brain ageing, but at a higher speed. Hence this cognitive decline has been termed Accelerated Cognitive Ageing (ACA; Breuer et al., 2016b). As the patients in this subgroup often suffer other brain pathology before the onset of epilepsy, the decline is 'cascadic' and represents a double hit phenomenon: where the first ' $h$ it' $^{\prime}$ makes the brain vulnerable but still recovers due to cognitive reserve capacity, and the second ' $h i t^{\prime}$ irreversibly damages the brain and its cognitive capacity, and leads to ACA (Breuer et al., 2016a).. More specifically a clear global cognitive deterioration takes place in relatively short period of time after the (usually late) onset of epilepsy (the second hit) on individuals with an already reduced reserve capacity from a traumatic brain injury, or (cardio)vascular diseases (the first hit).

The neurological mechanism of action of ACA is poorly understood. Functional MRI studies can shed light on such mechanisms and can give information on which brain areas or networks are involved, by comparing functional networks in ACA patients to a control group. In our previous work we showed that Granger causality can be used in neuroimaging to assess causality between time series of the fMRI signals (dynamic functional connectivity) (Bernas, Aldenkamp, and Zinger, 2018). Indeed, in several neuropsychiatric disorders, such as ADHD, autism or depression, effective dynamics appeared to be a powerful tool in describing neuronal correlates of such pathology (Bernas, Aldenkamp, and Zinger, 2018; Huang et al., 2017; Zhenyu Liu et al., 2012). In the present study, we use the same technique, i.e. dynamic functional connectivity (Granger causality) in an attempt to find relevant mechanisms of action of the ACA epileptic co-morbidity, more specifically in the dynamics between cognitive-related functional brain networks.

\subsection{Methods}

\subsubsection{Participants and cognitive measures}

Fourteen adult outpatients with a confirmed diagnosis of epilepsy and ACA were recruited from the centre for epilepsy Kempenhaeghe (Heeze, the Netherlands). For a description of the diagnostic process and the clinical characteristics of the included patients see (Breuer et al., 2019). The ACA patients were consecutively age- and education-matched with 16 healthy controls with no history of neurological or psychiatric illness. This study was approved by the Local Medical 


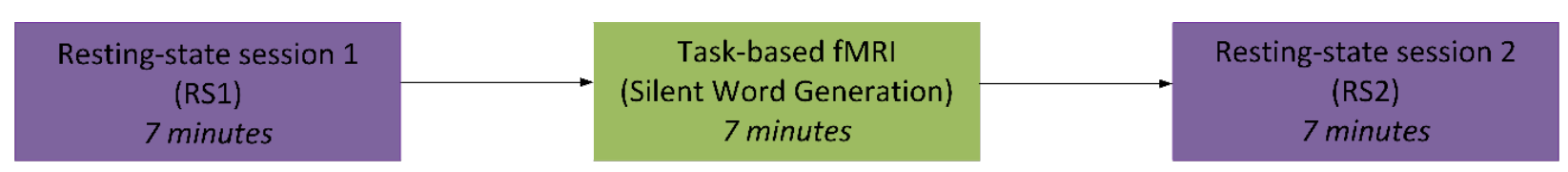

FIGURE 3.1: MRI protocol.

Ethical Committee. All subjects signed informed consent. 70\% of ACA patients had at least one co-morbidity, in which $57 \%$ were cerebrovascular, and $43 \%$ cardiovascular co-morbidities. After checking and pre-processing the fMRI-data, four ACA-patients and two healthy controls were excluded due to excessive head motion $(n=3)$, MRI artefacts $(n=2)$ and an unexpected detection of a brain tumour $(n=1)$. The effective sample therefore consisted of 10 ACA-patients and 14 healthy controls. Main demographic and clinical data are shown in Table 3.1. Importantly, deterioration scores were calculated by subtracting the estimated premorbid IQs (OPIE-IV scores (Holdnack et al., 2013) from the actual IQs, resulting in three IQ-deterioration scores: det-FSIQ, det-VCI and det-PRI.

\subsubsection{MRI protocol}

T1-weighted anatomical images were acquired using a 3.0 T imaging system (Philips Achieva) with a 3D Fast Field Echo (FFE) sequence: echo-time $(\mathrm{TE})=3.8 \mathrm{~ms}$, repetition time $(\mathrm{TR})=8.4$ ms; field of view $(F O V)=240 \mathrm{~mm} \times 240 \mathrm{~mm}$, with 180 sagittal slices; and voxel size $=1 \times 1 \times 1$ $\mathrm{mm} 3$; with SENSE factor of 1.5, and scan duration of $9 \mathrm{~min}$. All subjects underwent a series of functional MRI sessions: two resting-state (RS) fMRI scans and one task-based scan with an activation paradigm in between (silent word-generation task). The first resting-state fMRI scan (RS1) provided the baseline functional architecture in both subject groups. The following task-based fMRI-scan served as a means of initiating cognitive (task-based) activity. Data of this scan were not further analysed. The second resting-state scan (RS2) revealed whether functional connectivity changed after cognitive effort. FMRIdata were acquired using multi-echo echo-planar imaging (ME-EPI) sequences with 3 echoes: TEs $=12,35,58 \mathrm{~ms}$, TR $=2 \mathrm{~s} ; \mathrm{FA}=90$ deg.; SENSE factor = 2.7; 208 dynamics for a total of 7 minutes; 27 axial slices (with no gap), $64 \times 64$ matrix FOV, with a $3.5 \times 3.5 \times 4.5 \mathrm{~mm}^{3}$ voxel size. Figure 3.1 shows the MRI protocol the participants underwent. In this study only RS1 and RS2 are analysed, compared between the groups, and correlated with ACA psychological deterioration scores, as explain in the next sessions.

\subsubsection{Preprocessing and resting-state network extraction}

First, data were preprocessed to be denoised and normalised. We used the pipeline of Kundu et al., to clean all fMRI data of each individual and each session (Kundu et al., 2012). Briefly, these steps were co-registration to the T1-weighted anatomical images, followed by a co-registration of MNI152 space. Also slice timing correction, and head-motion correction was applied. The benefit of using multi-echo being that one can model the $\mathrm{T} 2 *$ decay. Using this $\mathrm{T} 2 *$ maps, a weighting on each voxel and for each echo is done and the echoes are then optimally combined. This maximises the BOLD SNR. However, noise is still present in these normalised fMRI data, and non-BOLD signal remains. Hence the multi-echo (ME) Independent Component Analysis (ICA) cleaning is performed (Kundu et al., 2017). Independent components are ranked through a goodness-of-fit method, to be split into BOLD and Non-BOLD components. After regression of the bad components upon the optimally combined-echo fMRI scans, multi-echo cleaned resting-state fMRI data are obtained for each participant/session (Evans et al., 2015). This ME-ICA cleaning has been 
TABLE 3.1: Demographic, clinical and neuropsychological characteristics of the subjects

\begin{tabular}{|c|c|c|}
\hline & ACA & Healthy controls \\
\hline Age in years $-M(s t d)$ [range] & $61.3(8.9)$ [50-74] & $62.2(9.8)[47-79]$ \\
\hline Gender & $70.0 \%$ male & $35.7 \%$ male \\
\hline Handedness & $100.0 \%$ right-handed & $92.9 \%$ right-handed \\
\hline Age at epilepsy onset & $35.0(14.6)$ [15-59] & - \\
\hline Duration of epilepsy & $22.3(15.2)[1-50]$ & \\
\hline Type of epilepsy & $\begin{array}{l}40.0 \% \text { cryptogenic localized } \\
40 \% \text { sympomatic } \\
20 \% \text { idopathic }\end{array}$ & - \\
\hline Dominant seizure type ${ }^{a}$ & $\begin{array}{l}10.0 \% \text { simple partial } \\
20.0 \% \text { complex partial } \\
20 \% \text { absence } \\
50 \% \text { seizure free }\end{array}$ & - \\
\hline Status epilepticus & $50.0 \%$ yes & - \\
\hline Seizure frequency & $\begin{array}{l}50.0 \% \text { seizure }(\mathrm{sz}) \text { free } \\
0.0 \%<1 \mathrm{sz} / \mathrm{y} \\
20.0 \% 1-5 \mathrm{sz} / \mathrm{y} \\
20.0 \% 1 \mathrm{sz} \text { per } 2 \text { months } \\
0.0 \% \text { montly sz } \\
10.0 \% \text { weekly sz } \\
0.0 \% \text { daily sz }\end{array}$ & - \\
\hline Drug load ${ }^{\mathrm{b}}$ & $1.0(0.4)$ & - \\
\hline \multicolumn{3}{|l|}{ WAIS-IV indexes } \\
\hline FSIQ & $76.7(8.7)^{*}$ & $108.4(13.4)$ \\
\hline VCI & $94.7(10.6)$ & $106.2(12.2)$ \\
\hline PRI & $75.8(6.9)^{*}$ & $103.2(13.8)$ \\
\hline WMI & $79.6(10.0)^{*}$ & $104.9(13.3)$ \\
\hline PSI & $67.0(14.4)^{*}$ & $114.6(8.7)$ \\
\hline \multicolumn{3}{|l|}{ OPIE-IV scores } \\
\hline OPIE-IV FSIQ & $98.8(9.2)$ & $109.6(12.4)$ \\
\hline OPIE-IV VCI & $95.2(11.7)$ & $105.6(14.6)$ \\
\hline OPIE-IV PRI & $96.8(9.3)$ & $102.5(12.4)$ \\
\hline \multicolumn{3}{|l|}{ Deterioration scores } \\
\hline det-FSIQ & $-22.1(5.0)^{*}$ & $1.3(8.2)$ \\
\hline det-VCI & $-0.5(8.9)$ & $0.2(6.0)$ \\
\hline det-PRI & $-21.0(4.5)^{*}$ & $0.0(8.7)$ \\
\hline \multicolumn{3}{|l|}{ Memory scores } \\
\hline Auditory & $93.9(9.7)^{*}$ & $109.9(12.6)$ \\
\hline Visual & $92.7(7.0)$ & $102.8(10.9)$ \\
\hline Delayed memory & $93.5(8.4)^{*}$ & $106.3(10.9)$ \\
\hline
\end{tabular}

${ }^{*} \mathrm{p}<.01$ significant difference between groups. WMI: Working Memoey Index. PSI" Processing Speed Index.

a Dominant seizure type is determined for the two years preceding neuropsychological assessment

$\mathrm{b}$ The prescribed daily dose of antiepileptic medication divided by the define daily dose (Lammers et al., 1995) 
proven to be the most robust de-noising methods for resting-state fMRI, and to improve substantially statistical power. (Dipasquale et al., 2017; Lombardo et al., 2016).

Second, in order to extract resting-state networks (RSNs), we temporally concatenated all the aforementioned ME-ICA cleaned scans (all participants and sessions), and applied the groupICA method (Beckmann and Smith, 2004) implemented in FSL ${ }^{3}$. The multi-echo cleaning preprocessing, described in the previous paragraph, found in average (among all participant/scans) 18 activity-related independent component (or networks). Therefore, we chose 18 degrees-offreedom for our group-ICA decomposition, to extract 18 group-RSNs. After the group-ICA, dualregression is used to obtain the subject-specifics components (RSNs) and their time series associated (Nickerson et al., 2017). After matching our RSNs maps with Smith and colleagues' brain template of the 10 most relevant resting-state networks, and after visual inspection, we selected 14 effective networks and 4 artefactual ones (see Figure 3.2.a) (Damoiseaux et al., 2006; Smith et al., 2009a).

\subsubsection{Granger causality and statistical analyses}

The selected RSNs have a time-series associated. These time courses are used to apply Granger causality (GC) for all pairs of RSNs under consideration. One signal $Y$ is said to Granger cause another signal $X$, if the past of $Y$ and $X$ can better predict the future of $X$ rather than with the past of $X$ only (Zaremba and Aste, 2014). The estimation of the F-statistics of GC strength, that describes directed functional connectivity, i.e. causality, between two networks time-series, were extracted using the MVGC toolbox (Barnett and Seth, 2014). This was applied on all participants' scan sessions. The terms 'causal', 'directed', 'dynamic', or 'effective' (functional) connectivity, represent the same concept of pairwise (between-network) GC, and are here interchangeable.

After obtaining each participants' GC adjacency matrix, where each entry represents a directed pairwise causal connection, a group comparison can be conducted. Since F values from GC were not normally distributed, we applied an exact (permutation) statistical testing, in order to find differences between ACA and Controls in their brain network causality. For all participants and the RSNs we applied 5000 permutations followed by 2-samples 1-sided t-tests. If our 'true' (ACA vs CON) T-score is greater than the 97.5 percentile of all the other T-scores, we can affirm significances in the difference between the cohorts (similar to having an alpha $=0.025$ in a parametric 2 sample 1-sided $t$-tests). This was repeated for both sides, to see when ACA $>C O N$ and when CON $>$ ACA in the between-networks causality strengths; and for the two resting-state scans (RS1 and RS2), to see differences or similarities between the two sessions, and get insights on the effect of the task-based fMRI that was acquired in-between.

Finally, we also correlated the psychological deterioration (det-) scores with the aforementioned GCs for all ACA participants. Deterioration scores are negatively signed, hence a positive correlation between det-scores and GC values would mean that with higher causality between two networks, the less deterioration is observed. Conversely, the more causality, the more deterioration, would be shown by a negative correlation. This correlation analysis was also performed on a setup wherein IC time-series from RS1 and RS2 were normalised and concatenated before undergoing the GC analysis described above. If effective connectivity of a pair of RSNs correlates with a deterioration score in all of the 3 setups (RS1, RS2, and RS1/RS2 concatenated), we have strong evidence that the pairwise directed connectivity is associated with the accelerated cognitive decline, no matter the resting states the participants are experiencing (pre-task RS1, or

\footnotetext{
${ }^{3}$ https://fsl.fmrib.ox.ac.uk/fsl
} 
post-task RS2). Hence, consistent results of significant correlations ensure reproducibility and independency of the results to the cognitive load or brain exhaustion state, making them strong predictors, or biomarkers of the ACA phenomenon.

\subsection{Results}

\subsubsection{Resting-state networks selection}

Using the group-ICA decomposition of FSL, we extracted 14 effective subject-specific spatial maps (RSNs) and their associated time series. Out of these 14 networks, 7 were relevant for our study. We retained the cognitive-related networks and removed sensory motor cortices and visual system networks. Furthermore, cerebellar networks were excluded. The 7 aforementioned networks used in our study are presented in Figure 3.2.b, and are comprised of the Default-mode network (DMN), the fronto-parietal right and left (FPR, FPL) networks, the Cingulate cortex network (CING), the Ventral and Dorsal Attention Networks (VAN, DAN) and the Salience Network (SN).

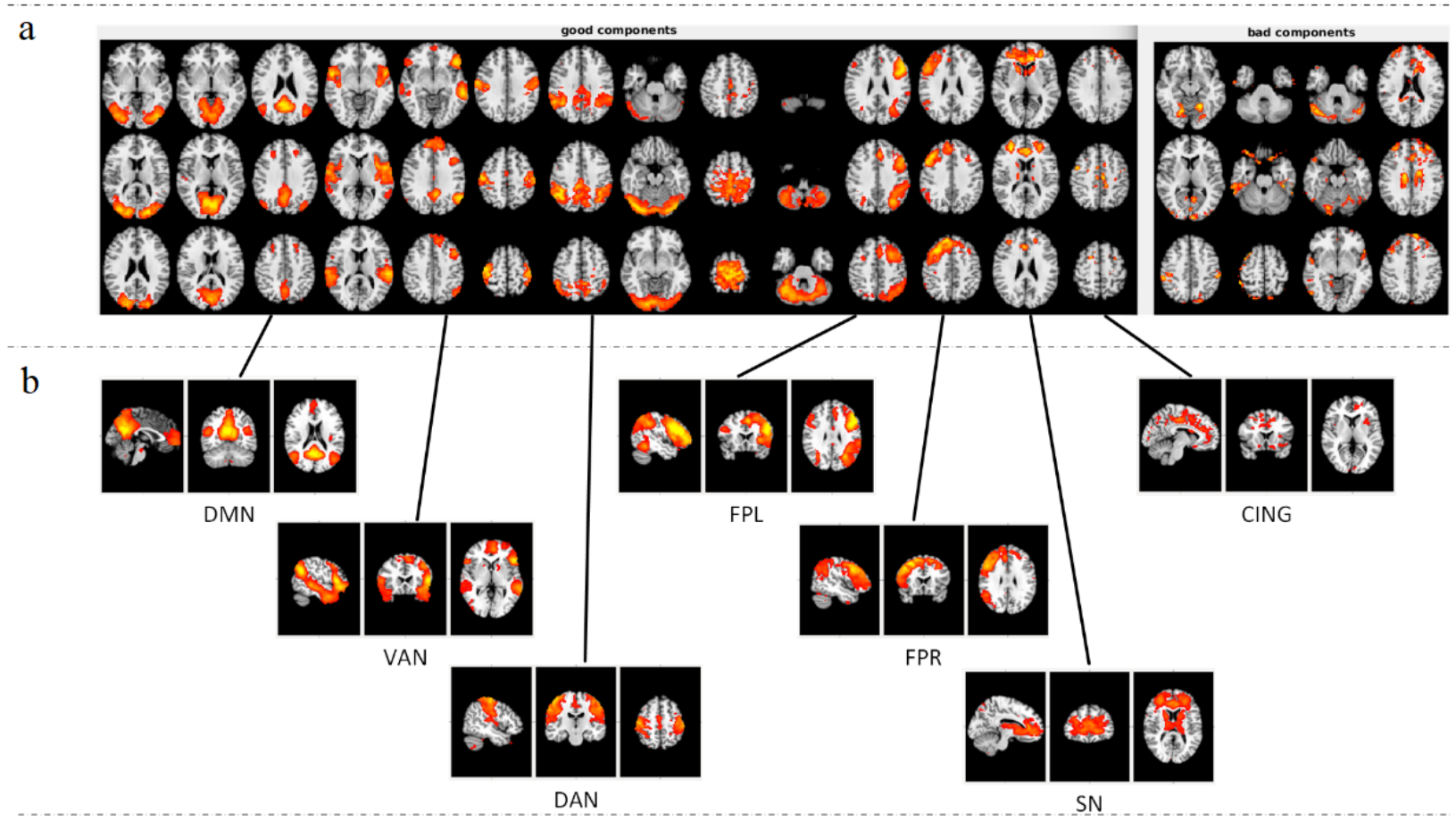

FIGURE 3.2: (a) Networks extracted from group-ICA, the 'good' networks on the left, and the 'bad' networks (noise) on the right. (b) Cognitive-related networks in which their activity time series (network fluctuations) are used for the Granger (dynamics) analysis.

\subsubsection{Granger causality differences}

Using the 7 relevant networks we applied Granger causality for all (42) pairs of networks and compared the strength of the effective connectivity between the groups. Figure 3.3 shows the results of the statistical comparison, for each resting-state scan separately.

In RS1, the ACA group displayed a stronger causal connectivity as compared to controls. For 3 directed effective connections from the cingulate cortex: CING $\rightarrow$ FPR, and CING $\rightarrow$ SN, CING $\rightarrow$ DAN; for an effective connectivity from the ventral attention network: VAN $\rightarrow$ FPL; and for 


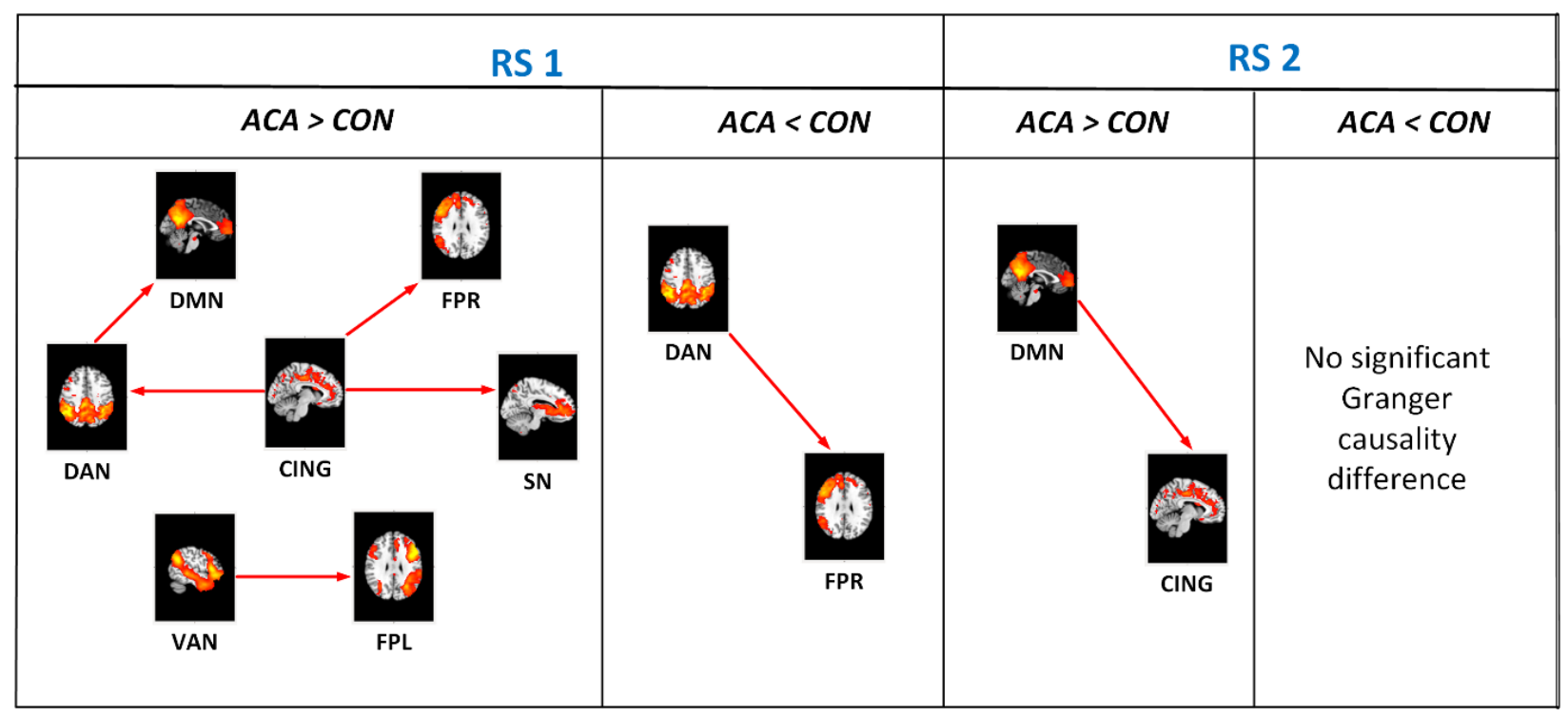

FIGURE 3.3: Pairs of networks that showed differences in causality strength between patients and controls, in resting-state session 1 and 2. Red arrows indicate the direction of the causal connectivity between networks.

the pair DAN $\rightarrow$ DMN. Also, in RS1, the ACA group showed a weaker dynamic connectivity for the directed connectivity DAN $\rightarrow$ FPR.

Regarding the second resting-state session ACA were significantly stronger in their effective connectivity only for the pair: DMN $\rightarrow$ CING. We summarised our strongest (lowest p-values) evidence of group difference results in pairwise granger causalities, involving the cingulate cortex, in Figure 5A

\subsubsection{Correlation between Granger causality and deterioration scores in ACA}

In a post-hoc analysis we calculated the (Pearson) correlations between the Granger causality values between pairs of networks, and their deterioration scores. Figure 3.4 depicts the statistically significant results (correlation p-value $<0.05$ ), per resting-state session. This was only performed for the ACA patients as deterioration scores in the controls were approximately 0 . The effective connectivity FPR $\rightarrow \mathrm{SN}$, and SN $\rightarrow$ CING correlated negatively with the deterioration in full scale IQ in RS1, whereas in RS2, DMN FPL (both directions), DAN $\rightarrow$ SN negatively correlates and SN $\rightarrow$ VAN positively correlates with det-FSIQ. For the RS1/RS2 concatenated time-series analysis, det-FSIQ positively correlates with the dynamics DMN $\rightarrow$ FPL, and negatively correlates with Granger causality SN $\rightarrow$ CING. Regarding correlation with det-PRI scores, in the 3 setups (RS1, RS2, RS1/RS2 concatenated) patients had a negative correlation with the effective connectivity $\mathrm{DMN} \rightarrow$ VAN. The dynamics FPL $\rightarrow$ SN showed positive correlation with deterioration scores, i.e., less FPL $\rightarrow$ SN causal connectivity would lead to greater deterioration.

The Granger causality from DMN to VAN was consistently associated with det-PRI in ACA patients, i.e. the effect was present in RS1, RS2, and the RS1/RS2 concatenation set-ups. This consistency was not found for det-FSIQ. Also, the decline in PRI is the most relevant psychological characteristics for diagnosing ACA. Therefore, the correlation between DMN $\rightarrow$ VAN GC and the det-PRI score is our main and most reliable significant result and is depicted in Figure 5B. 


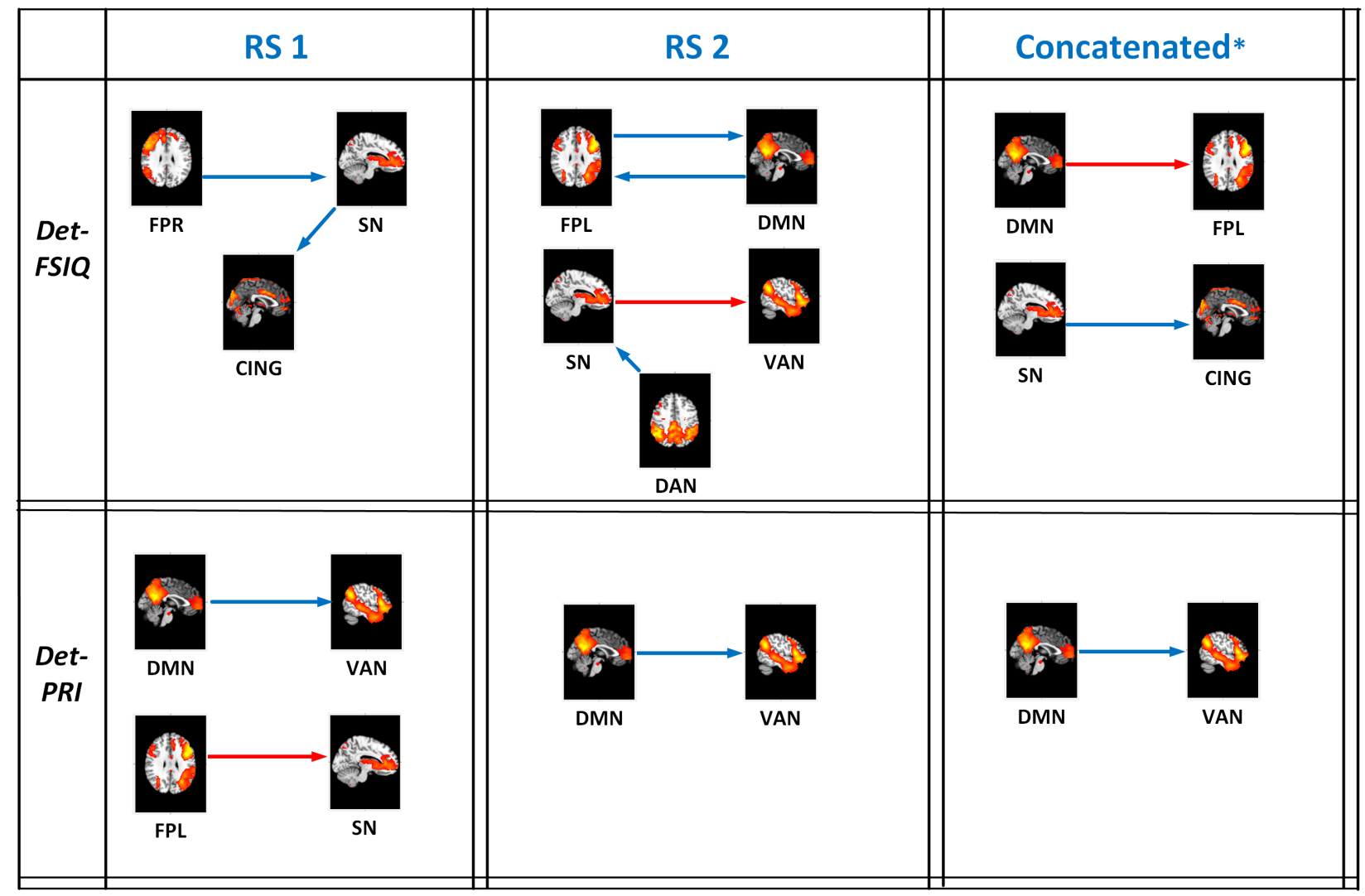

FIGURE 3.4: Significant correlation between the effective connectivity (Granger causality) and the psychological deterioration scores. Negative correlation (anti-correlation) in blue arrows; positive correlation in red arrows. Note: a negative correlation means that the more negative the deterioration score is, i.e. higher decline, the stronger the effective connectivity is. Whereas for a positive correlation: the higher the decline, the lower the effective connectivity strength is. 

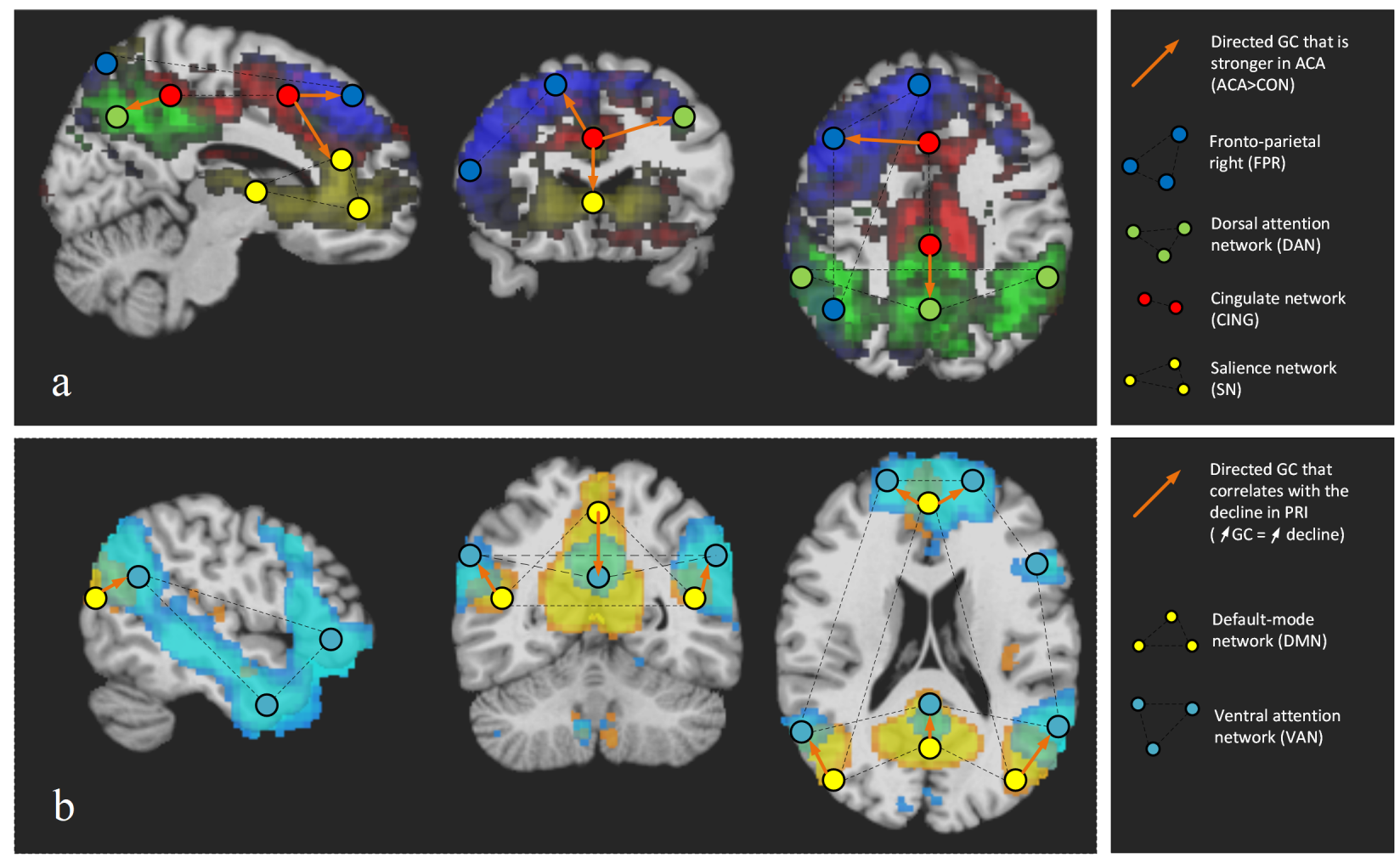

FIGURE 3.5: Summary results of GC in ACA. (a) Main evidence of abnormal connectivity in ACA, with increased GC between the cingulate cortex and three cognition-related RSNs; (b) and the most consistent (in RS1 and RS2) correlation between the GC and the decline in Perceptual Reasoning Index (det-PRI) in ACA.

\subsection{Discussion}

Many fMRI studies have identified age-related changes in within-network functional connectivity but few have reported an association of between-network connectivity and age-related cognitive deficits (Li et al., 2015; Sala-Llonch, Bartrés-Faz, and Junqué, 2015). Researchers have mainly focused on DMN connectivity, using correlation methods (Ferreira and Busatto, 2013). Also, decreased connectivity within the nodes of the main RSNs, including the DMN, salience, executive, and attention networks has been shown. These results has been observed using ICA (Damoiseaux et al., 2008; Onoda, Ishihara, and Yamaguchi, 2012b), seed-based connectivity (Andrews-Hanna et al., 2007), and graph-theory in a whole brain approaches (Song et al., 2014; Tomasi and Volkow, 2012). Similar research has been done in case of mild-cognitive impairment in elderly (FarràsPermanyer, Guàrdia-Olmos, and Peró-Cebollero, 2015). Few studies have investigated the effective connectivity within cognitive networks. In these studies, the effective connectivity within and between the DMN, dorsal attention networks and salience networks were studied in ageing (Ly et al., 2014; Tsvetanov et al., 2016). In our study, we conducted an effective connectivity analysis (Granger causality), not only for the DMN, DAN, and SN, but also the FPL, FPR, CING, and VAN (i.e., the executive-control networks). It is shown that the latter networks are the pillars of cognitive processing, and take part of the DMN, SN, and EXE triad useful for rest-task switching (Chen et al., 2013; Menon and Uddin, 2010; Onoda, Ishihara, and Yamaguchi, 2012b), and task flexibility (Chen et al., 2016). This task switching and flexibility is an important impairment in ACA patients (Breuer et al., 2016a; Breuer et al., 2019).

Our results suggest that ACA patients have stronger between-networks effective connectivity from the cingulate cortex compared to the age- and education-matched control participants. We 
showed that CING over-recruits the fronto-parietal (executive) networks (FPR, SN, DAN) in the baseline resting state fMRI (main group-difference results in Figure 3.5a). This is in line with the compensation-related utilization of neural circuit hypothesis (CRUNCH) model that has been proposed to describe the use of compensatory mechanisms in ageing (Schneider-Garces et al., 2010). Briefly, CRUNCH proposes that older adults recruit greater neural resources to compensate at a lower cognitive load but that at a higher cognitive load, older adults show equivalent or lower activation and worse cognitive performance compared with young adults (Li et al., 2015; ReuterLorenz and Cappell, 2008). This effect has been observed in the PFC and also in the parietal cortex, concretely in the precuneus and posterior cingulate and both in episodic memory tasks and in working memory tasks (Sala-Llonch, Bartrés-Faz, and Junqué, 2015). Our DAN, FPR, and SN are clearly the constituent networks of the PFC and parietal cortex and are more strongly causally connected from the CING in RS1 as compared with controls. Hence, we may hypothesise that ACA patients recruit greater neural resources to compensate as seen at the baseline rest (RS1, low cognitive load). However, after cognitive activation (task-based fMRI), such compensatory mechanisms fail and in the second resting state scan (after a cognitive task) ACA patients showed equivalent or lower activation as compared with healthy elderly. The over-recruitment of CING towards the other cognitive networks is also supported by the study of Cao et al. where ageing has proven to have an effect on the resting-state functional connectivity in anterior cingulate cortex, with increased connection with salience network parts (CING $\rightarrow \mathrm{SN}$ ) but reduced integration with DMN (Cao et al., 2014). Also, the increased dynamic connectivity from CING and the decreased connectivity DAN $\rightarrow$ FPR in ACA can be explained by the Posterior-Anterior Shift with Aging (PASA) model (Davis et al., 2008). PASA hypothesises deficits in ageing to activate regions in the posterior midline cortex - as for our reduced DAN $\rightarrow$ FPR — accompanied with increased activity in medial frontal cortex - as for our CING-related dynamics. In this regard, this posterior-anterior shift commonly results in the disconnection between the anterior and the posterior nodes of the DMN, which correlates with age-related cognitive decline (Andrews-Hanna et al., 2007; Damoiseaux et al., 2008). More research has shown evidence that ageing reduces within-DMN connectivity and increases connectivity between the DMN and external regions (Sala-Llonch, Bartrés-Faz, and Junqué, 2015). The within-network decrease and between-network increase of connectivity can be seen as a decreased network segregation, which occurs across the healthy adult lifespan (Chan et al., 2014; Wig, 2017). We also found an over-recruitment of the effective connection DAN $\rightarrow$ DMN in RS1, that can be explained by the decreased anti-correlation between DMN and DAN proven to occur in ageing at rest (Spreng et al., 2016).

The posterior-anterior shift caused by the disruption within the DMN, is in line with our correlations between effective connectivity and the det-FSIQ. Indeed, the PASA model could explain that a weakened DMN $\rightarrow$ FPL and a strengthened SN $\rightarrow$ CING were linked to stronger IQ declines. The short-range increase of connectivity between the SN and CING seems however to contradict the findings of Onoda et al. (Onoda, Ishihara, and Yamaguchi, 2012a), but is in line with other research (Sala-Llonch, Bartrés-Faz, and Junqué, 2015; Tomasi and Volkow, 2012). Indeed the salience network has proven to relate to cognitive capacity, where the within-salience connectivity was anti-correlated with fluid intelligence, and multitasking (Tsvetanov et al., 2016). This closely resembles the short-range increased connectivity SN $\rightarrow$ CING that correlated with the decline in cognition (FSIQ deterioration). Moreover, when concatenating RS1 and RS2 network time series, ACA showed a strong positive correlation between DMN $\rightarrow$ FPL and the det-FSIQ, i.e., more directed connectivity from DMN to FPL would lead to less FSIQ deterioration in ACA. This is in line with the recent findings that a strengthened DMN-LFC (left frontal cortex) connectivity supports reserve (i.e., a relatively preserved cognition in disproportion to the extent of neuropathology) in mild cognitive impairment and ageing (Franzmeier et al., 2017, 2018). Indeed, for our ACA patients, a weaker DMN $\rightarrow$ FPL leads to a stronger cognitive deterioration, as if ACA patients' reserve capacity was not able to maintain a relatively preserved cognition. This is also in line with 
our cascadic hypothesis, and lack of cognitive reserve after the second 'hit' in ACA.

IQ decline in the Perceptual Reasoning Index (PRI) is mainly correlated with DMN and the (ventral) attention network dynamics. More specifically, the stronger the Granger causality from DMN to VAN there is, the more deterioration in fluid IQ is expected (Figure 3.5.b). It has been shown that between-network connectivity with DMN is challenged with ageing, and it is associated with sharpening of the boundaries of the default mode network, and integration of the insula and cingulate with fronto-parietal attentional regions (Anderson et al., 2011). This is similar to our det-PRI correlation with the SN $\rightarrow$ CING dynamic. Anderson and his colleagues also showed decreasing correlation between the default mode and attention control networks with age, which is in line with our consistent DMN $\rightarrow$ VAN dynamics that correlated with the deterioration in PRI (Anderson et al., 2011).

\subsection{Limitations}

In this study three major limitations are to be mentioned. First, we acknowledge that a third group of patients with epilepsy but no ACA, would have strengthened the results interpretation. Some of the (GC) effects depicted in ACA patients might be apparent because of epilepsy itself. And adding such a group could better confirm that effect seen here are solely made by the ACA comorbidity. Only few recent studies could show impaired directed causal inferences in brain connectivity (Klugah-Brown et al., 2019; Li et al., 2019; Zweiphenning et al., 2019). The impaired directed connectivity was epilepsy-type dependent, and located in epileptic tissues, in an EEG study (Zweiphenning et al., 2019); or was different from healthy controls in the subcorticalcortical connectivity, in frontal lobe and temporal lobe epilepsy (Klugah-Brown et al., 2019; Li et al., 2019). The latter studies were also using resting-state fMRI but were limited in trying to shed light on impaired mechanism in focal epilepsies with significant results related to specific clinical characteristics (consciousness-impairing seizure in Li et al., 2019 and duration of epilepsy in Klugah-Brown et al., 2019). Those characteristics are not relevant in our ACA case, where the cognitive decline is of importance. ACA patients are more heterogeneous in their epilepsy forms, seizure types, refractoriness, and durations (see Table 3.1), so our results should not reflect the effects of a specific type of epilepsy. Additionally, ACA cannot be separated from Epilepsy, since it is defined as an epileptic co-morbidity. Even though cognitive decline is seen in other degenerative disorders, such as in epilepsy dementia or Alzheimer disease, in such cases, degeneration do not only impaired fluid intelligence, but also long-term memory. We are not aware of other ageing disorder that develop similar, and quite specific, cognitive decline comorbidity.

As a second limitation, we hypothesised that the cognitive task executed prior a resting-state has an effect on the resting state the brain experienced, which is debatable. However we want to emphasise we do not hypothesise that the task itself and its evoked dynamics could persists at rest afterwards, but rather, that brain cognitive reserve capacity is challenged (Bastin et al., 2012; Franzmeier et al., 2018); and that brain fatigue could still persist for a few minutes right after executing a cognitively demanding task (Ren et al., 2019). So that the speed of communication, and the use of the (activity-related) network resources, weakened after the task. It also happens for healthy controls, but at lesser degree, as found in our study.

Thirdly, Granger causality validity in fMRI data is still debated. However a research group simulated fMRI data and found out that GC at the fMRI level is monotonically related to GC at the neural level, i.e., GC from BOLD signals reliably detect neural GC (Wen, Rangarajan, and Ding, 2013). Hence, they concluded that neural activity (or here, neural GC) changes, such as in a pathological group, can be detected from the fMRI data. But in our case, time-series are not direct BOLD 
signals, but indirect (BOLD-derived) network activity, so it remains uncertain that the monotonic relationship between neural GC and the network GC is fully preserved.

Overall, caution should be taken with respect to the interpretation of the effect of (network) brain dynamics on the IQ decline, and further research with a third group, an epilepsy-control group, could alleviate these limitations and uncertainties.

\subsection{Conclusion}

Accelerated cognitive ageing (ACA) in epilepsy shows decline in cognitive abilities that resemble normal processes of cognitive ageing in older subjects, however at greater speed (hence: 'accelerated cognitive ageing'). Comparing brain resting-state dynamics of ACA patients and age-, and education-level-matched controls, we mainly found strengthened effective connectivity between large-scale cognitive networks in ACA. This shows a tendency of over-recruitment as a compensatory mechanism, in line with the $\mathrm{CRUNCH}$ model for ageing. However, this seem to be a fragile mechanism that fails after cognitive load, showing an exhaustion phenomenon, corroborating with our second-hit model, which describes the lack of cognitive reserve capacity in ACA patients. Furthermore, the DMN to ventral attention network directed connectivity strongly correlates with the decline in the perceptual reasoning IQ, which is in line with the PASA model. Both CRUNCH and PASA models show that the deterioration in the patients can be described as processes that are seen in older individuals albeit, in our patients at a younger age. 

Chapter 4

Brain resting-state networks in adolescents with high-functioning autism: Analysis of spatial connectivity and temporal neurodynamics

[Published in Brain and behavior] 


\begin{abstract}
Autism Spectrum Disorder (ASD) is mainly characterised by functional and communication impairments as well as restrictive and repetitive behaviour. The leading hypothesis for the neural basis of autism postulates globally abnormal brain connectivity, which can be assessed using functional Magnetic Resonance Imaging (fMRI). Even in the absence of a task, the brain exhibits a high degree of functional connectivity, known as intrinsic, or resting-state, connectivity. Global default connectivity in individuals with autism versus controls is not well characterised, especially for a high-functioning young population. The aim of this study is to test whether high-functioning adolescents with ASD (HFA) have an abnormal resting- state functional connectivity.

We performed spatial and temporal analyses on Resting-State Networks (RSNs) in 13 HFA adolescents and 13 IQ- and age- matched controls. For the spatial analysis, we used probabilistic Independent Component Analysis (ICA) and a permutation statistical method to reveal the RSN differences between the groups. For the temporal analysis, we applied Granger causality to find differences in temporal neurodynamics.

Controls and HFA display very similar patterns and strengths of resting-state connectivity. We do not find any significant differences between HFA adolescents and controls in the spatial restingstate connectivity. However, in the temporal dynamics of this connectivity, we did find differences in the causal effect properties of RSNs originating in temporal and prefontal cortices.

The results show a difference between HFA and controls in the temporal neurodynamics from the ventral attention network to the salience-executive network: a pathway involving cognitive, executive- and emotion-related cortices. We hypothesised that this weaker dynamic pathway is due to a subtle trigger challenging the cognitive state prior to the resting state.
\end{abstract}




\subsection{Introduction}

Autism Spectrum Disorder (ASD) is a heterogeneous neurodevelopmental disorder, which is characterised by persistent deficits in social communication and social interaction across multiple contexts and restricted, repetitive patterns of behaviour, interest or activities (DSM-V). Although not part of the diagnostic classification and not formal subcategories of ASD, a distinction is also often made between low-functioning autism (LFA) and high-functioning autism (HFA). No consensus criteria regarding LFA and HFA exist, but high-functioning individuals with autism tend to have a 'normal' IQ (Barendse et al., 2013). Recently, increasing interest has been focused on abnormalities in (functional)organisation of specific brain regions, or networks, related to cognitive functions such as working memory, executive function, visual attention and language processing (Anderson et al., 2011; Cherkassky et al., 2006).

Many task-based fMRI studies report that ASD is associated with either weaker or stronger connectivity between various structures (Monk et al., 2009). However, focusing on the 'resting-state' (i.e. task free) in fMRI provides a different domain to measure cortical synchronisation patterns. Indeed, in the past decade, functional connectivity of resting-state fMRI data is rapidly emerging as a highly efficient and powerful tool for in vivo mapping of neural circuitry in the human brain (Zuo et al., 2010). Thus far, resting-state functional connectivity MRI studies in autism provide inconsistent results, i.e., showing either under- or hyper- connectivity in similar investigated brain regions (Monk et al., 2009; Müller et al., 2011; Rane et al., 2015; Uddin, Supekar, and Menon, 2013). But those studies mainly assessed within-network connectivity, i.e., between hubs/ROIs connectivity. And evidence shows that for adolescents and adults, the impaired connectivity is to be found between, rather than within, large-scale networks (Bos et al., 2014; Nomi and Uddin, 2015; Redcay et al., 2013; Tyszka et al., 2014).

To extract those large-scale resting-state networks and their associated time series, neuroimaging researchers have adopted a multivariate signal processing method known as Independent Component Analysis (ICA). This data-driven method needs no a priori on the measured signals, and hence, really suitable for resting-state analysis (Beckmann et al., 2005. Although ICA can provide spatial and temporal information about anatomical regions that show similar functional connectivity, it does not reveal causal relationships between components, i.e. the effectiveness directionality and strength - of the connectivity (Deshpande et al., 2009; Liao et al., 2010).

And recent evidence suggests that not the topology (structural and functional maps), but rather the dynamics of the network can better describe the disorder (Chen et al., 2016; Deshpande et al., 2013; Hanson et al., 2013; Kana et al., 2014; Wicker et al., 2008). Hence, we also extract causality measures, which we call 'temporal neurodynamics' in this paper, to represents temporal causal effect dependencies between RSNs. Temporal neurodynamics can be visualised using the WienerGranger causality test (Bressler and Seth, 2011; Granger, 1969), and its derived causality magnitude $\mathrm{F}$ upon two brain signals (time series). Here, the time series represent the RSN low-frequency oscillations, extracted from ICA. Therefore, in this study, we focus on large-scale networks, their shape and strength (within-network spatial connectivity), and their effective connectivity with other large-scale networks (between-network neurodynamics). Regarding the potentially impaired networks, we also focus only on networks involving saliency, executive function, ventral attention network and the default mode network, as those well-known networks have shown atypical connectivity within and between networks. (Anderson, Ferguson, and Nielsen, 2013; Keown et al., 2017; Nomi and Uddin, 2015).

Finally, we also test, using two resting-state scans (rs-scan 1 and 2) and a 1-back visual task-based fMRI in-between, the hypothesis that a task-based fMRI scan prior to a resting-state scan session influences the post-task resting-state connectivity (Barttfeld et al., 2012; Hassan Saleh, 2011). 
Indeed we could expect a change in neurodynamics, and brain flexibility, after a cognitively demanding task in ASD, while controls would recover faster and should show none or less significant between-resting-state scans changes. Therefore, not only between-group difference is analysed but also the between-scan (recovery) effects (within the groups). Differences between HFA and controls may be present in the composition of the spatial network organisation (connectivity) and/or in the temporal neurodynamics (causal effect).

\subsection{Methods}

\subsubsection{Participants}

13 adolescents with ASD and 13 age- and IQ-matched controls participated in this study. All participants were between 12 and 18 years old. Individuals with ASD were recruited from De Berkenschutse, a special secondary education school in Heeze (the Netherlands). All adolescents in the control group were recruited through an advertisement in a (local) newspaper and visited regular secondary schools in various regions of the Netherlands. Written informed consent was also obtained from the next of kin, caretakers, or guardians on behalf of the adolescents enrolled in this study. Inclusion criteria for the adolescents with HFA were: established diagnostic criteria according to the DSM-IV, as well as the autism algorithm cut-offs on the Autism Diagnostic Observation Shedule (ADOS) (Barendse et al., 2013; Bildt et al., 2009) . Inclusion criteria for the control group were: no history of psychiatric illness. Adolescents in the control group were excluded if they and/or one of their siblings and/or parent(s) had a diagnosis of ASD. Further exclusion criteria for both groups were: a comorbid psychiatric disorder; a significant hearing or visual impairment; an inability to speak/understand the Dutch language; and/or a comorbid central neurologic or other somatic disorder.

Table 4.1 shows the means and standard deviations (SD) of the ages in months and the Wechsler scores: the Verbal Comprehension Index (VCI), Perceptual Organization Index (POI), Freedom from Distractibility Index (FDI) and Full Scale Intelligence Quotient (FSIQ). Using the Analysis of Variance (ANOVA) statistical method, we assessed the differences of the conditions (intelligence scores) of the both groups.

The study protocol was approved by the Medical Ethical Commission of the Maastricht University Medical Centre.

\subsubsection{Image acquisitions}

MRI was performed on a 3.0-Tesla unit (Philips Achieva) equipped with an 8-channel receiveronly head coil. For anatomical reference, a T1-weighted 3D fast (spoiled) gradient echo sequence was acquired with the following parameters: repetition time (TR) $8.2 \mathrm{~ms}$, echo time (TE) $3.7 \mathrm{~ms}$, inversion time (TI) $1022 \mathrm{~ms}$, flip angle $8^{\circ}$, voxel size $1 \times 1 \times 1 \mathrm{~mm}^{3}$, field of view (FOV) $240 \times 240$ $\mathrm{mm}^{2}, 150$ transverse slices. Then, resting-state fMRI data was acquired using the whole brain single- shot multi-slice BOLD echo-planar imaging (EPI) sequence, with TR 2 s, TE $35 \mathrm{~ms}$, flip angle $90^{\circ}$, voxel size $2 \times 2 \times 4 \mathrm{~mm} 3$, matrix 128x128, 32 contiguous transverse slices per volume, and 210 volumes per acquisition; resulting in total resting-state acquisition of 7 minutes.

The resting-state scans were performed twice with an 8-minutes lasting 1- back test for working memory assessment in-between. This 1-back test was performed to assess the working memory processes. For this memory task, pictures of houses or faces (neutral and smiling faces) were displayed randomly at regular intervals. Then, patients and controls were asked to indicate when 
the current stimulus (pictures) matched the previous picture (Koshino et al., 2008; for more details on the 1-back task, see Figure A.2 and A.3 in Appendix A. For the both resting-state scans, participants were instructed to lie with their eyes closed, to think of nothing but not to fall asleep.

\subsubsection{Data preprocessing}

Data analysis was carried out using FMRIB Software Library $\left(\mathrm{FSL}^{1}\right)$. The following preprocessing was applied (Kruijs et al., 2014) discard of the first 3 volumes $(=6 \mathrm{~s})$ allowing the magnetization to reach equilibrium; rigid-body motion correction (Jenkinson et al., 2002) non-brain tissue removal; slice-timing correction; registration to the Montreal Neurological Institute (MNI) standard space (2 $\mathrm{mm}$ isotropic); spatial smoothing using a Gaussian kernel of $4.0 \mathrm{~mm}$ full-width at half-maximum (FWHM); grand-mean intensity normalisation; and high-pass temporal filtering at $100 \mathrm{~s}(0.01 \mathrm{~Hz})$. After these preprocessing steps one autistic subject and the second scan of a control participant were rejected because of a too large head motion: absolute displacement (mean) $>1 \mathrm{~mm}$ with a maximal relative displacement (between two consecutive slice) $>3 \mathrm{~mm}$. Also, we reported the average framewise displacement (FD) in $\mathrm{mm}$ for each group and rs-scan in Table 4.1.

\subsubsection{Group independent component analysis}

A single group-level ICA was performed across all subjects and all scans from both HFA and control groups using probabilistic ICA as implemented in FSL Multivariate Exploratory Linear Optimized Decomposition into Independent Components (MELODIC). First, the previously preprocessed $4 \mathrm{D}$ dataset was temporally transformed by concatenation into a single time series. This new 4D image was, then, separated into 34 independent components (ICs). The number of components was arbitrarily set to 34 as it seems to be a good trade-off to get a sufficient number of relevant networks (around ten), without splitting them into subcomponents (Wang et al., 2011) To obtain the components, group probabilistic ICA processing steps were applied to the temporally concatenated 4D image: masking out non-brain voxels, voxel-wise demeaning of the data, and normalisation of the voxel-wise variance. Subsequently, the pre-processed data were projected into a 34-dimensional subspace using probabilistic Principal Component Analysis. Then these observations were decomposed into sets of vectors which describe signal variations across the temporal domain (time-courses), the session/subject domain and the spatial domain (maps) by optimising for non-Gaussian spatial source distributions using a fixed-point iteration technique (Hyvärinen, 1999). The resulting estimated component maps were divided by the standard deviation of the residual noise and threshold at a posteriori probability threshold of $p>0.5$ (i.e., an equal loss is placed on false positives and false negatives) by fitting a Gaussian/gamma mixture model to the histogram of intensity values (Beckmann et al., 2005).

\subsubsection{Resting state networks selection}

The most relevant group-IC maps (out of 34) were selected according to the following 3 steps. First, group-IC maps with more than $33 \%$ of the estimated spectral power in high-frequencies $(>0.1 \mathrm{~Hz}$ ) were excluded to keep only networks within the low frequency range of $0.1-0.01 \mathrm{~Hz}$ (Lowe, Mock, and Sorenson, 1998; Tyszka et al., 2014) Secondly, Smith and colleagues described the major co-varying networks in the resting brain and created a template of these RSNs widely used in resting-state fMRI studies (Smith et al., 2009a). With this template and our remaining group maps, a function, using the 'goodness-of-fit' approach was created and applied (Greicius

\footnotetext{
$1_{\text {Ww. fmrib.ox.ac.uk/fsl }}$
} 
et al., 2004; Vanhaudenhuyse et al., 2010). Finally, the third step consisted in a visual inspection of each component spatial profile to verify the consistency and ensure the effectiveness of the two previous steps. Plus, this last step allows us to select other known and well-described networks that are not in Smith and colleagues' template, but still comply with first selection step.

\subsubsection{Spatial RSN analysis between groups}

The first level of the voxel-wise group analysis was performed using dual-regression (Beckmann et al., 2009). The aim of this process is to obtain, from the group-IC maps, subject-specific IC maps. Dual-regression involves two General Linear Models (GLM). First, the group-IC maps were used as spatial regressors against the preprocessed individual fMRI scans. This results in singlesubject time courses for each component separately. Then, these time courses were normalised to unit variance to test both the "shape" and "amplitude" of the RSN. In the second GLM, these normalised individual time courses were used as temporal regressors against the preprocessed individual fMRI images, leading to subject-specific IC maps for each subject's scan. As there were two subject-specific spatial maps per IC for each individuals (one per scan), before running final group-level analysis, we merged and averaged these two IC maps per subject. We also compared the two groups for each scan separately, i.e., without the merging and averaging of the RSN maps, as explained below.

The second level of the group analysis consisted in getting the effects of within-group means (control group average $>0$; HFA group average $>0$ ) and between-group differences (HFA > control; control > HFA). This was assessed using non-parametric permutation testing (5000 permutations), with FSL's Randomise tool (Nichols and Holmes, 2002) For each RSN, the resulting statistical maps were threshold at $\mathrm{p}<0.05$ family-wise error (FWE) corrected with the threshold-free cluster enhancer (TFCE) technique (Smith and Nichols, 2009). Finally nuisance regressors describing age, IQ and relative gray matter volume were added to the model in a second experiment, to observe their possible effects on the between-group contrast maps.

\subsubsection{Temporal dynamics of RSNs}

The statistical Granger causality (G-causality) allows us to assess causality among two signals. One signal $Y$ is said to Granger cause another signal $X$, if the past of $Y$ and $X$ can better predict the future of $X$ rather than with the past of $X$ only (Zaremba and Aste, 2014) In this study we use this principle to evaluate pairwise multivariate conditional Granger causalities of our independent components (resting-state networks). The assessment is performed on each pair of subject-specific RSNs time series and repeated for each resting-state scan, using the Multivariate Granger Causality (MVGC) toolbox (Barnett and Seth, 2014). This gives us estimates F of Granger causality magnitudes for each network pairs, subject, and scan. Further, with 2-sample two-tailed t-tests we compare these G-causality magnitudes between the two groups (HFA vs controls) to determine different patterns of neuronal dynamics of the resting-state (effective connectivity). We also perform this test for the two resting-state scan sessions to assess whether or not, a previous task-based fMRI scan can trigger and/or change the dynamics of resting-state connectivity, i.e., the causality between RSNs). Finally, we assess if these changes differ between patients and controls. According to several studies, people with ASD show differences mainly in frontal and temporal cortices, default mode parts, and also within networks related to social interaction (Anderson, Ferguson, and Nielsen, 2013; Hanson et al., 2013; Keown et al., 2017; Nomi and Uddin, 2015) Therefore, we selected the RSNs located mainly in fronto-temporal cortices and/or consisting of socio-cognitive brain parts. These networks are assessed and compared with the method described above. 
TABLE 4.1: Demographic and descriptive data of ASD and control adolescents

\begin{tabular}{|c|c|c|c|c|}
\hline Measure & $\begin{array}{l}\text { ASD } \\
\text { M (SD) }\end{array}$ & $\begin{array}{l}\text { Controls } \\
\text { M (SD) }\end{array}$ & $\begin{array}{l}\text { Difference } \\
\mathrm{F}(1,24)\end{array}$ & $\mathrm{p}^{*}$ \\
\hline Gender & $12 \mathrm{~m} ., 1 \mathrm{f}$. & $12 \mathrm{~m} ., 1 \mathrm{f}$. & - & - \\
\hline Ages (years) & $15.3(1.2)$ & $14.5(1.3)$ & 2.89 & .102 \\
\hline Verbal comprehension index & $117.1(9.0)$ & $117(10.4)$ & 0.00 & .968 \\
\hline $\begin{array}{l}\text { Perceptual Organization } \\
\text { index }\end{array}$ & $114.0(5.8)$ & $109(7.8)$ & 4.85 & $.038^{*}$ \\
\hline $\begin{array}{l}\text { Freedom from Distractibility } \\
\text { index }\end{array}$ & $99.5(14.5)$ & $101.9(14.6)$ & 0.19 & .670 \\
\hline Full-scale IQ & $116.7(5.0)$ & $113.2(7.8)$ & 1.92 & .179 \\
\hline $\begin{array}{l}\text { Autism Diagnostic } \\
\text { Observation Schedule } \\
\text { (number of patients) }\end{array}$ & $\begin{array}{l}2(6) \\
1(5) \\
0(2)\end{array}$ & $0(13)$ & - & - \\
\hline \multicolumn{5}{|l|}{ Framewise displacement (mm) } \\
\hline $\begin{array}{l}\text { Scan } 1 \\
\text { Scan } 2\end{array}$ & $\begin{array}{l}0.089(0.043) \\
0.082(0.034)\end{array}$ & $\begin{array}{l}0.092(0.034) \\
0.079(0.033)\end{array}$ & $\begin{array}{l}0.063 \\
0.033\end{array}$ & $\begin{array}{l}0.80 \\
0.86\end{array}$ \\
\hline
\end{tabular}

a 2 autistic disorder, $1=\mathrm{ASD}$, and $0=$ no diagnosis according to ADOS. ${ }^{*} \mathrm{~A}$ p-value $<0.05$ means that a score or a characteristic (rows of the table) differs significantly between the two cohorts

\subsection{Results}

For each group, age, gender, intelligence scores, ADOS diagnostic score and mean framewise displacement (in $\mathrm{mm}$ ) per group and per scan, are displayed in Table 1. Only the Perceptual Organization Index (POI) score showed a significant difference between the adolescents with ASD and the controls ( $\mathrm{p}<0.05$, Tab. 1).

\subsubsection{RSN selection and between-groups spatial analysis}

We extracted functionally relevant group ICs based on the resting-state template from (Smith et al., 2009a). After visual inspection, we also included the widely described ventral attention network (Corbetta, Patel, and Shulman, 2008; Farrant and Uddin, 2015; Fox et al., 2006). Finally for further analysis, we described our executive control network as the salience-executive network, since it involves, not only prefrontal and posterior cingulate cortices (for executive function), but also the salient network compounded with the anterior insular and anterior cingulate cortices (Menon and Uddin, 2010; Sala-Llonch, Bartrés-Faz, and Junqué, 2015). The 11 networks that we finally obtained are depicted in Figure 4.1. Those RSNs were found in both HFA and controls by testing the subject-specific maps of these networks (after the dual regression)In those relevant networks, the group effects (group mean $>0$ ) are present and strongly consistent with the whole group (ASD + Controls) networks (see Figure A.1 in Appendix A) Also, after a non-parametric permutation test (5000 permutations) threshold at $\mathrm{p}<0.05$, TFCE corrected for FEW,no voxels, in any components, were significant in the second-level group analysis for the ASD > control and control > ASD contrasts. The same results occurred when comparing the groups for each scan seperately. In the second equivalent statistical analysis, where age, IQ level and gray matter density were added as covariates, again, no voxels in any component survived at the same threshold $(\mathrm{p}<0.05$, FWE corrected; see Table A.1 in Appendix A for more details). Hence, statistically, the strength and the extent of each network (functional connectivity) was similar in both groups. 


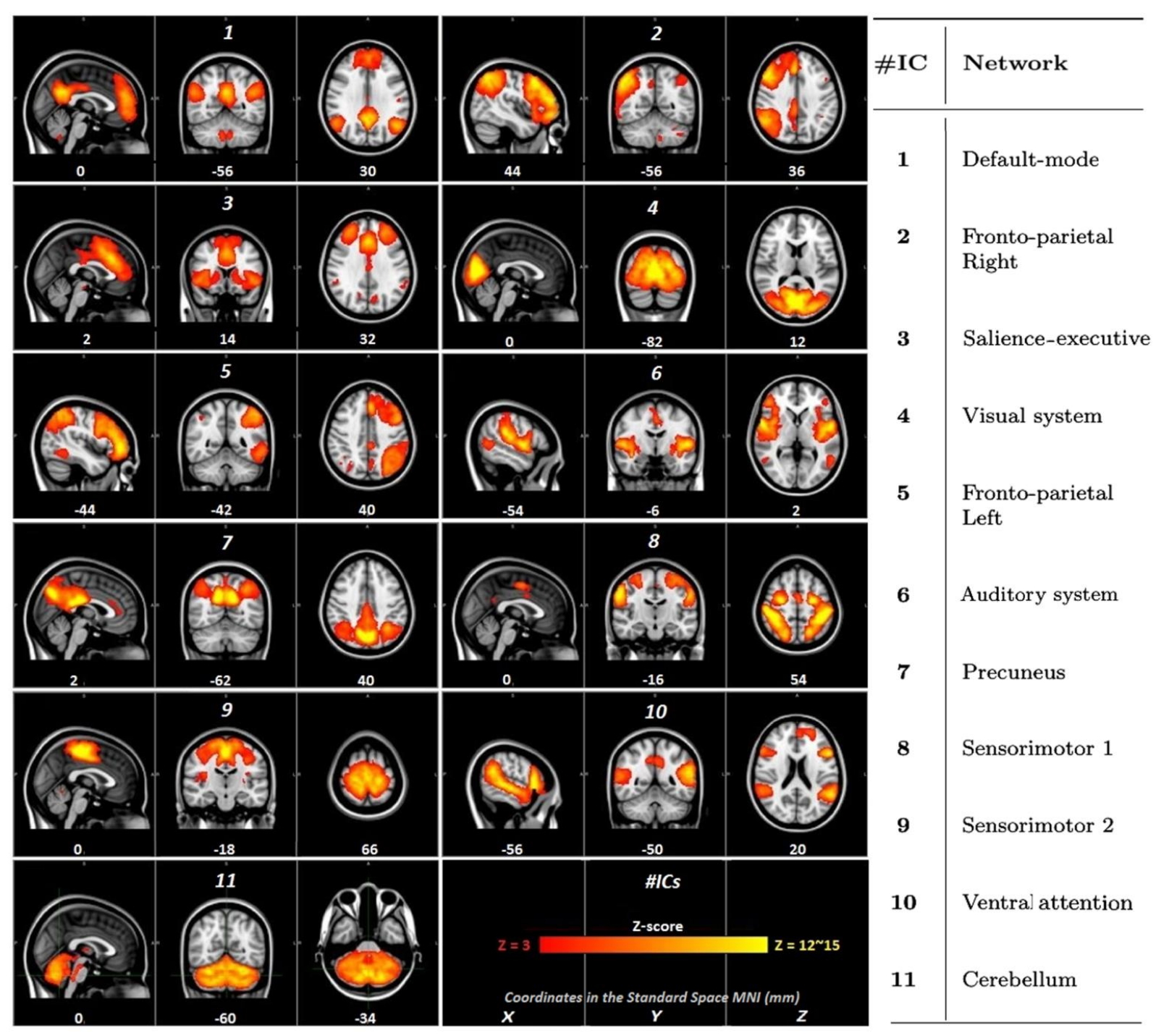

FIGURE 4.1: Relevant components extracted from the group-level ICA. Relevant components extracted from the 34 group-IC maps overlaid in colour on the MNI standard brain $(2 \times 2 \times 2 \mathrm{~mm})$. Names of the networks are in the right-side table. Colorbar is thresholded between 3 and 15 (z-score). MNI coordinates are in $\mathrm{mm}$. The left hemisphere corresponds to the right side in the images (radiological convention). 


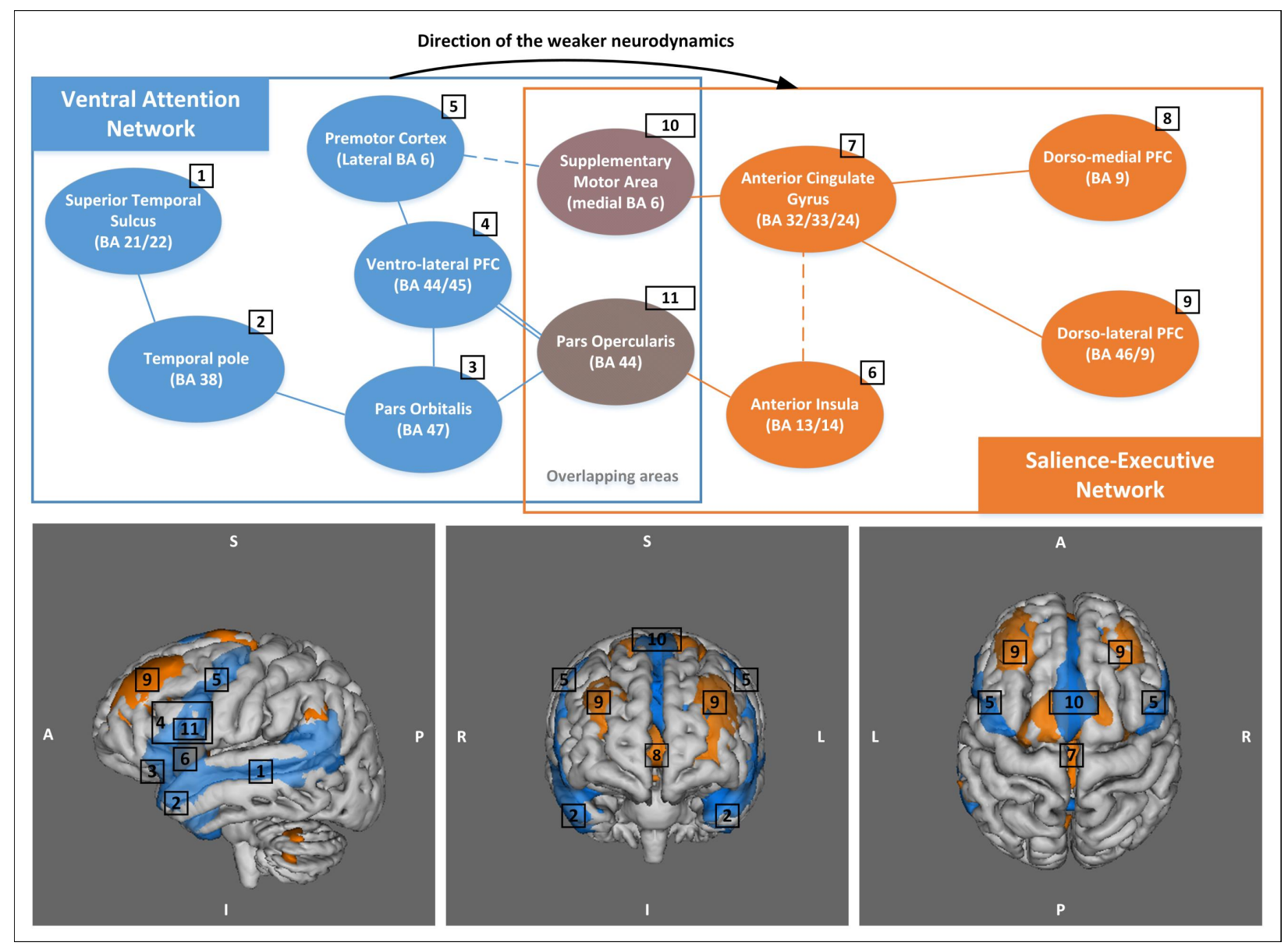

FIGURE 4.2: Visualisation of the weaker neurodynamic pattern in adolescent with HFA (in the post-task resting state). Visualisation of the two RSNs, salience-executive in blue and ventral attention in orange, where the causal dynamics is weaker in HFA for the second (post-task) resting-state scan, in the direction from ventral attention to salience-executive. The scheme above shows the different cortices involved in these two networks and the overlapping areas. Solid lines and dashed lines describe direct cortico-cortical physical link and indirect connections (through white matter and/or basal ganglia), respectively. The pars opercularis is a part of the ventro-lateral PFC which is shown with the double line. On the bottom part, anatomical visualisation of the two abovementioned RSNs (from group-IC contrast maps) are displayed (threshold at z-score $>2.6$, i.e., p-value $<0.01$ ). BA = Brodmann Area; PFC =Prefrontal Cortex. 


\subsubsection{Temporal dynamics of the RSNs}

The causal analysis to detect temporal dynamics differences was made upon the 4 most relevant RSNs that involved fronto-parietal and temporal cortices, and networks related to social cognition (Anderson, Ferguson, and Nielsen, 2013; Hanson et al., 2013; Keown et al., 2017; Nomi and Uddin, 2015). Therefore we selected the default mode network (IC 1, Figure 4.1), the salienceexecutive system (IC 3), the ventral attentional network (IC 10) and the auditory system (IC 6). Pairwise conditional Granger-causality magnitudes, in average, within each group for the $1^{\text {st }}$ and $2^{\text {nd }}$ resting-state scan sessions, were significant ( $\mathrm{p}<0.05$ FDR corrected). This was found for the 4 aforementioned selected prefrontal and temporal RSNs and for both groups. Also, the positivity of the normality test (Kolmogorov-Smirnov) for the distribution of the causalities among each group, allowed us to use the 2-sample 2-tailed t-test to compare HFA adolescents' G-causalities with those of the control group.

In the $1^{\text {st }}$ resting-state can, none of the pairwise causalities differed significantly between ASD and controls. We also found no significant differences in causality within the control group, i.e., when comparing $1^{\text {st }}$ rs-scan and $2^{\text {nd }}$ rs-scan. However, dynamic RSN patterns did differ within the ASD cohort ( $1^{\text {st }}$ rs-scan vs $2^{\text {nd }}$ rs-scan) and significantly diverged from control adolescents only in the $2^{\text {nd }}$ resting-state scan. The latter result shows a significant lower value of Granger-causality between the ventral attention and salience-executive networks in the ASD group as compared with control: Mean F (Granger causality value) for ASD $=0.028$ ( $\mathrm{SD}=0.015)$; Mean F controls = $0.058(\mathrm{SD}=0.031) ; \mathrm{t}(24)=3.17$, $\mathrm{p}$-value $=0.0042)$. Figure 4.2 shows this directed causal connection and displays in more detail the cortical regions involved in these two networks.

\subsection{Discussion}

In the present study of high-functioning adolescents with autism, resting- state whole-brain functional connectivity was examined. No evidence was found for any significant difference in brain spatial connectivity between the two populations. However, our results did show that patterns in temporal neurodynamics, i.e. causal effects of one RSN on another, differ between the groups. In contrast with controls, HFA display a significant difference in temporal neurodynamics between resting-state fMRI sessions 1 and 2. Furthermore, in contrast with the first resting-state scan, temporal neurodynamics differ significantly between HFA and Controls during the second resting-state session. The primary findings of similar functional connectivity between the two cohorts challenge the theory that the autistic brain is globally underconnected (Belmonte, 2004; Uddin, Supekar, and Menon, 2013). This can be explained by differences in scan protocols, in post-processing methods (ICA vs seed-based) and mainly because of the population (type of the ASD, number and ages). However, our findings of similar functional resting-state network, i.e., similar within-network functional connectivity are corroborated by other studies on adolescents and adults with high-functioning autism (Bos et al., 2014; Tyszka et al., 2014; Uddin, Supekar, and Menon, 2013). More recently, Nomi and Uddin, 2015 also showed that adolescents with ASD do not have altered within-network functional connectivity. But interestingly, by means of correlation between the RSNs time series, they obtained evidence of impaired between-network connectivity in the adolescents with autism. The pairwise temporal correlations used in their study can be seen as (undirected) instantaneous causality. Hence, their results of between-network hypoconnectivity in ASD population is partially (only instantaneous causality) in line with our results of weaker neurodynamics in autism, which are discussed in the next paragraph.

To go further and detect strength and directionality in causality between RSNs, we used Granger causality upon 4 relevant socio-cognitive RSN time series. The two RSNs showing differences in 
effective directed connectivity (neurodynamics) are the ventral attention network and the salienceexecutive control network. The ventral attention contains mainly the left and right superior temporal sulci (STS), the temporal poles, the ventro-lateral and orbital cortices, and lateral premotor cortex. This pathway is known to code for visual recognition and identification, and for emotional processes. The temporal pole is known to play a role in functions that tend to be weak in autism: social and emotional processing, including face recognition and the theory of mind (Kana et al., 2015; Olson, Plotzker, and Ezzyat, 2007). Also, the STS has been postulated to be a critical component of the abnormal neural circuitry underlying deficits in social perception in autism (Redcay, 2008). The STS projects information towards prefrontal cortices (mainly the medial and lateral) which are part of the salience-executive network. This salience-executive control network involves the anterior cingulate gyrus (ACC), the anterior insular cortex (AI) as well as the dorsomedial and dorso-lateral prefontal cortices, and the supplementary motor area (SMA). These ROIs are involved in cognitive processes such as working memory, reasoning, task flexibility, problem solving, planning and execution (Chan et al., 2008) The AI cortex is a brain structure implicated in disparate cognitive, affective and regulatory functions, including interoceptive awareness, emotional responses, and empathic processes (Menon and Uddin, 2010). More specifically, Dapretto proposes a mirror neuron system (MNS) dysfunction in children with ASD (Dapretto et al., 2006). Notably, they affirm that the MNS activity in the pars opercularis is consistently present during imitation, action observation, and intention understanding; and this pars opercularis combined with the insula and limbic activity (e.g., in the ACC) may mediate the understanding of others' emotional states. However, the absence of mirror neuron activity in the frontal part of the MSN (pars opercularis) leads this emotional process to be weaker in ASD. This weakened 'Theory of Mind' network has been further confirmed in children and adolescents (Kana et al., 2015.

The association AI/ACC, also termed the salient network, plays a role in dynamic switching between brain networks in reaction to cognitively demanding tasks (switch default mode network / executive network; Menon and Uddin, 2010; Sridharan, Levitin, and Menon, 2008). A review study reports that this critical system (salience network) is impaired in ASD, and that the AI region has demonstrated hypo-activity in individuals with ASD across a wide variety of social cognitive task paradigms (Di Martino et al., 2009b; Uddin and Menon, 2009) All these findings illustrate that activations in brain areas implicated in the ventral attention and salience-executive RSNs are known to be weaker in the ASD population. Also, areas in the salient network and the MNS, i.e., the causal flow 'bridge' area (Figure 4.2), are critical during self- and other-related social and affective processes, and also known to be under-activated in ASD (Barttfeld et al., 2012; Kana et al., 2015). In line with the previously mentioned studies conducted with the help of socioemotional cognitive task-based fMRI, we observed the same weaknesses in whole brain restingstate functional connectivity, but only when analysing temporal dynamics. The extracted RSNs do not have the same pattern of temporal dynamics, i.e., the influence of one RSN on another varies between the two cohorts: the causal connectivity between the salience-executive and ventral attention networks (in the direction of ventral attention $\rightarrow$ salience-executive) is significantly weaker in the HFA population, but only in their post-task resting state. This impaired temporal neurodynamics suggests failing bridging of the emotional states regulated in the ventral attentional to the decision-making-oriented salience-executive control system. This may therefore be described as a more rigid system in terms of the emotional-executive bridge, which can be seen as an endogenous-to-exogenous (self-to-other) dynamic process failure as suggest by the literature (Di Martino et al., 2009a; Ebisch et al., 2011; Menon and Uddin, 2010; Uddin et al., 2011; Uddin et al., 2013). Finally, in our study, the differences in temporal neurodynamics were only found in the second resting-state session.An 1-back working memory task-based scan was performed in between the two resting-state scan sessions. We therefore hypothesise that abnormal temporal neurodynamic patterns in HFA were triggered by the working memory task, involving not only working memory, but also attentional and emotional (in terms of face and emotion recognition) 
processes. This could be explained by a reduced cognitive flexibility (or more rigidity) in the posttask resting-state connectivity in ASD, reducing the between-networks dynamics, showing a more brain state dependency of connectivity pattern in autism compared to controls, as shown recently in literature (Barttfeld et al., 2012; Chen et al., 2016; Douw et al., 2016; Uddin et al., 2015)

\subsubsection{Limitations}

One of the main challenges in applying G-causality upon fMRI BOLD signals is the problem of the Hemodynamic Response Function (HRF) changes. Inter- regional HRF variation has been argued to affect G-causality analysis (David et al., 2008). But the Granger causality method implemented in MVGC software, used in our study, has been proven to be robust to changes in HRF properties (Seth, Chorley, and Barnett, 2013). A second limitation with our technique is the relatively long sample intervals (TR) of classic fMRI protocols (usually ranging from $1 \mathrm{~s}$ to $3 \mathrm{~s}$ ). Indeed, our TR of $2 \mathrm{~s}$ is substantially longer than typical inter-neuron delays. However, since we examine changes (of differences) in G-causality rather than attempting to find a ground truth G-causality pattern, that limitation is not significant (Barnett and Seth, 2014).

Finally, even though our statistical analyses are properly controlled for multiple comparisons and for type I error (false positive), cautious interpretation of the results is in order. Especially misses (type II error, or false negative), could have occurred for the results of similar spatial network connectivity (miss of spatial differences).

\subsubsection{Methodological recommendations}

For future application, we state that neurodynamics provide alternative strategies when ICA analysis does not yield differences for a cross-sectional analysis. Also, conversely, where ICA does show differences in functional connectivity between two populations (or more), we advise not to use Granger causality analysis on temporal trends of ICs, but rather on raw ROI signals (with same ROI location for both groups). Finally, our findings show that tasks prior to resting-state acquisition scan can have an effect on the results of an effective connectivity analysis.

\subsection{Conclusion}

We find no significant differences in resting-state brain connectivity between high-functioning adolescents with ASD and the control group at the whole- brain level. However, the extracted RSNs do not have the same pattern of temporal dynamics, i.e., the influence of one RSN on another is different between the two cohorts. In particular, the causal connectivity between the salience-executive and the ventral attention networks (in the direction of ventral attention $\rightarrow$ salience-executive) is significantly weaker in the HFA population in the $2^{\text {nd }}$ resting-state scan, after challenging sensitive functions for HFA adolescents. These two networks link cortices coding for face/object recognition and emotional processing with cortices of executive cognitive functions (attention, control, working-memory, behaviour). We hypothesise that changes in neurodynamics at rest in HFA are subtly triggered by challenging the cognitive state prior to the resting-state. And these changes seem to appear in the dynamic connectivity between the networks functionally related to the previous cognitive task. 
Chapter 5

\section{Wavelet coherence-based classifier: a resting-state functional MRI study on neurodynamics in adolescents with high-functioning austism}




\begin{abstract}
The autism spectrum disorder (ASD) diagnosis requires a long and elaborate procedure. Due to the lack of a biomarker, the procedure is subjective and is restricted to evaluating behaviour. Several attempts to use functional MRI as an assisting tool (as classifier) have been reported, but they barely reach an accuracy of $80 \%$, and have not usually been replicated or validated with independent datasets. Those attempts have used functional connectivity and structural measurements. There is, nevertheless, evidence that not the topology of networks, but their temporal dynamics is a key feature in ASD. We therefore propose a novel MRI-based ASD biomarker by analysing temporal brain dynamics in resting-state fMRI.

We investigate resting-state fMRI data from 2 independent datasets of adolescents: our in-house data (12 ADS, 12 controls), and the Leuven dataset (12 ASD, 18 controls, from Leuven university). Using independent component analysis, we obtain relevant socio-executive resting-state networks (RSNs) and their associated time series. Upon these time series we extract wavelet coherence maps. Using these maps, we calculate our dynamics metric: time of in-phase coherence. This novel metric is then used to train classifiers for autism diagnosis. Leave-one-out cross validation is applied for performance evaluation. To assess inter-site robustness, we also train our classifiers on the in-house data, and test them on the Leuven dataset.

We distinguished ASD from non-ASD adolescents at 87.5\% accuracy (91.7\% sensitivity, 83.3\% specificity). In the second experiment, using Leuven dataset, we also obtained the classification performance at $86.7 \%$ ( $83.3 \%$ sensitivity, and $88.9 \%$ specificity). Finally, we classified the Leuven dataset, with classifiers trained with our in-house data, resulting in $80 \%$ accuracy $(100 \%$ sensitivity, $66.7 \%$ specificity).

This study shows that change in the coherence of temporal neurodynamics is a biomarker of ASD, and wavelet coherence-based classifiers lead to robust and replicable results and could be used as an objective diagnostic tool for ASD.
\end{abstract}




\subsection{Introduction}

Autism spectrum disorder (ASD) is diagnosed in more than $1 \%$ of the world population. For example in the U.S., 1 in 68 children has an ASD (Centers for Disease Control and Prevention, 2016). This neurodevelopmental disorder is mainly characterised by social communication impairments as well as restrictive and repetitive behaviour. A distinction is often made between low-functioning and high-functioning autism - the latter tending to have an IQ higher than 80. Over the past decade, ASD has been studied using fMRI, in setups where participants had to perform a specific task or during resting-state fMRI, in order to find a biomarker for autism. Unfortunately, current literature provides mixed and inconsistent results regarding strength and location of functional disparities (Anderson, Ferguson, and Nielsen, 2013; Müller et al., 2011; Rane et al., 2015; Tyszka et al., 2014). Yet, several attempts to classify autism, based on functional connectivity measurements have been made (Anderson et al., 2011; Nielsen et al., 2013). In a review paper, Plitt and his colleagues show that functional connectivity classification using resting-state fMRI can be done with statistical significance (Plitt, Barnes, and Martin, 2015). However, such a classifier falls short of biomarker standards such as high classification accuracy and with a proven multi-sites robustness: peak accuracy of $76.7 \%$ for resting-state fMRI based methods. In another study, using seed-based connectivity measurements between regions of interest, an accuracy of $79 \%$ ( $83 \%$ sensitivity, and $75 \%$ specificity) is obtained, and $71 \%$ accuracy is achieved in a replication dataset (Anderson et al., 2011). Finally, using large-scale networks, Uddin and her colleagues reached $78 \%$ classification accuracy ( $75 \%$ sensitivity, $80 \%$ specificity) using solely the salient network connectivity, and could classify an independent dataset at $83 \%$ accuracy $(67 \%$ sensitivity, 100\% specificity)(Uddin et al., 2013).

In this paper, we propose a novel approach to find a biomarker of autism. There is evidence that not the topology of networks, but their temporal dynamics is a key feature in ASD (Chen et al., 2016; Hanson et al., 2013; Kana et al., 2014; Wicker et al., 2008). Our approach aims at assessing the temporal dynamics processes in the brain, which we call neurodynamics - quantifying, in our study, interactions between functional networks over time. We found only few fMRI studies that reports abnormal brain dynamics in adults with autism in terms of effective connectivity, i.e. the causal influence of one brain area on another. Nevertheless these studies were performed between regions of interest during explicit emotion-related tasks and show atypical effective connectivity patterns in social brain networks (Hanson et al., 2013; Wicker et al., 2008). We could not find any study dealing with effective connectivity, or other dynamics measurements, between or within large-scale functional networks at rest.

Wavelet decomposition and wavelet transform coefficients represent a powerful tool to assess transient, or non-stationary processes, and get insights in brain temporal dynamics. Cross-wavelet transform provides information of localised (in time and frequency) correlations between temporal signals (Torrence and Compo, 1998). It is also possible to derive from the wavelet transforms the phase shift between two signals. Combining these methods, we can extract wavelet-based coherence maps as originally performed in geophysics (Grinsted, Moore, and Jevrejeva, 2004) . In neuroscience, the wavelet transforms have been recently applied mainly as a filtering tool or transient detector in EEG studies (Catarino et al., 2013; Indic and Narayanan, 2011) . Regarding resting-state fMRI, only few exploratory studies have been conducted in order to show nonstationarity in brain resting-state network fluctuations (Chang and Glover, 2010; Damaraju et al., 2014).

In this study, we explore the coherence maps to extract our novel metric 'time of in-phase coherence'. This metric describes in-phase and coherent patterns (synchronicity) between pairwise large-scale resting-state functional networks. To our knowledge, this is the first study of temporal dynamics between brain networks evaluating pairwise synchronicity using wavelet coherence. 
TABLE 5.1: Demographic and clinical characteristics of the two datasets.

\begin{tabular}{lllll}
\hline & ASD Mean $(\mathrm{SD})$ & Controls Mean $(\mathrm{SD})$ & F-test (df1, df2) & P-value \\
\hline In-house dataset & $\mathrm{N}=12(12 \mathrm{M})$ & $\mathrm{N}=12(12 \mathrm{M})$ & $\mathrm{F}(1,22)$ & - \\
Ages (years) & $15.5(1.0)$ & $14.4(1.3)$ & 2.51 & .127 \\
VCI $^{\mathrm{a}}$ & $117.1(9.0)$ & $117(10.4)$ & 0.00 & .968 \\
POI $^{\mathrm{b}}$ & $114.0(5.8)$ & $109(7.8)$ & 4.85 & $.038^{*}$ \\
FDI $^{\mathrm{c}}$ & $99.5(14.5)$ & $101.9(14.6)$ & 0.19 & .670 \\
FSIQ $^{\mathrm{d}}$ & $116.7(5.0)$ & $113.2(7.8)$ & 1.92 & .179 \\
Leuven dataset & $\mathrm{N}=12(9 \mathrm{M} / 3 \mathrm{~F})$ & $\mathrm{N}=18(14 \mathrm{M} / 4 \mathrm{~F})$ & $\mathrm{F}(1,28)$ & - \\
Ages (years) & $13.7(1.2)$ & $14.4(1.6)$ & 1.96 & .172 \\
VIQ $^{\mathrm{e}}$ & $92.9(18.8)$ & $114.1(12.7)$ & 13.58 & $.001^{*}$ \\
PIQ $^{\text {f }}$ & $101.9(14.7)$ & $106.1(8.6)$ & 0.98 & .33 \\
\hline
\end{tabular}

${ }^{a}$ Verbal Comprehension Index, ${ }^{b}$ Perceptual Organization Index, ${ }^{c}$ Freedom from Distractibility Index, ${ }^{d}$ Full Scale IQ, ${ }^{\text {e }}$ Verbal IQ, ${ }^{\text {f }}$ Performance IQ. ${ }^{*}$ Statistically significant

We also present our classification results for distinguishing ASD from healthy adolescents, using our wavelet-based dynamics features.

\subsection{Methods}

\subsubsection{Participants}

Two independent samples were used in this study. In our in-house sample, 15 adolescents with ASD and 18 age- and IQ-matched controls initially participated in this study. Due to signal distortions caused by their braces, 2 participants with ASD and 5 control individuals were excluded from data analysis. Further in the study, an adolescent with ASD was also excluded because of an inaccurate brain registration during the preprocessing of the resting-state fMRI data, leading to 12 participants in each group for our in-house dataset. To retest our classification approach, as well as for validating classifiers using an independent dataset, we used resting-state fMRI data from University of Leuven in Belgium, available on the Autism Brain Imaging Data Exchange ${ }^{1}$. This dataset was chosen based on the similarity with our in-house data, i.e. matching in age (all adolescents) and having a sufficiently 'normal' IQ (> 80). After preprocessing of Leuven sample, we excluded 5 participants (bad registration and/or too large head-motion), leading to 18 control adolescents and 12 ASD. In both datasets, participants were selected from a clinical sample of children with previous diagnosis made by a multidisciplinary team including a pediatric neurologist/psychiatrist and based on the DSM-IV-TR criteria. To meet these criteria, we used the Autism Diagnostic Observation Schedule (ADOS) scores, whereas in Leuven sample, the Social Communication Questionnaire (SCQ) and Social Responsiveness scale (SRS) were used. IQ scores were, in both samples, assessed using the Dutch Wechsler Intelligence Scale for Children (WISC-III). Table 5.1 shows the descriptive data and psychological scores of the two samples, and Analyses of Variance (ANOVA) F-test results showing significant differences (if $\mathrm{p}$-value $<0.05$ ).

Finally, in both datasets, written informed consent was obtained from the next of kin, caretakers, or guardians on behalf of the adolescents enrolled in this study. Our in-house protocol was approved by the Medical Ethical Commission of the Maastricht University Medical Centre.

\footnotetext{
${ }^{1}$ http://fcon_1000.projects.nitrc.org/indi/abide
} 


\subsubsection{Data acquisition}

Our in-house dataset, MRI acquisition was performed on a 3.0-Tesla unit (Philips Achieva) equipped with an 8-channel receiver-only head coil. For anatomical reference, a T1-weighted 3D fast (spoiled) gradient echo sequence was acquired with the following parameters: repetition time (TR) $8.2 \mathrm{~ms}$, echo time (TE) $3.7 \mathrm{~ms}$, inversion time (TI) $1022 \mathrm{~ms}$, flip angle $8^{\circ}$, voxel size $1 \times 1 \times 1 \mathrm{~mm}^{3}$, field of view (FOV) $240 \times 240 \mathrm{~mm}^{2}, 150$ transverse slices. Then, resting-state fMRI data were acquired using the whole brain single-shot multi-slice BOLD echo-planar imaging (EPI) sequence, with TR $2 \mathrm{~s}$, TE $35 \mathrm{~ms}$, flip angle $90^{\circ}$, voxel size $2 \times 2 \times 4 \mathrm{~mm}^{3}$, matrix $128 \times 128,32$ contiguous transverse slices per volume, and 210 volumes per acquisition; resulting in total a resting-state acquisition of 7 minutes.

In the dataset from Leuven University, MRI data were acquired with a 3.0-Tesla unit (Philips Intera) also equipped with an 8-channel receiver head coil. Anatomical T1-weigthed images were 3D fast field echo sequences with $\mathrm{TR}=9.6 \mathrm{~ms}$ and $\mathrm{TE}=4.6 \mathrm{~ms}$, flip angle $8^{\circ}$, voxel size $0.98 \times 0.98 \times 1.2$ $\mathrm{mm}^{3}$, field of view (FOV) 250x250 $\mathrm{mm}^{2}, 182$ Coronal slices $(218 \mathrm{~mm})$. Regarding the resting-state fMRI, a multi-slice FFE single-shot EPI was acquired with the following parameters: TR $1656 \mathrm{ms,}$ TE $33 \mathrm{~ms}$, flip angle $90^{\circ}, 3.59 \times 3.59 \times 4.0 \mathrm{~mm}^{3}$ with FOV 230x230 $\mathrm{mm}^{2}$, and 32 transverse slices (128 $\mathrm{mm}$ ) per volume, and 250 volumes, resulting in a total scan time of $7 \mathrm{~min}$ and $6 \mathrm{~s}$.

For the resting-state scans, participants were instructed to lie with their eyes closed, to think of nothing but not to fall asleep. Also, in both datasets, the resting-state scans were acquired under similar conditions.

\subsubsection{Resting-state ICA time-series extraction}

In order to conduct the wavelet coherence classification using resting-state time series, we first extracted spatial RSN maps, using a group Independent Component Analysis (gICA) on restingstate fMRI preprocessed dataset. First the following preprocessing steps were applied using FEAT (a tool from FMRIB Software Library ${ }^{2}$ ): discarding the first 3 volumes allowing the magnetisation to reach equilibrium; rigid-body motion correction; non-brain tissue removal; slicetiming correction; registration to the Montreal Neurological Institute (MNI) standard space (2 $\mathrm{mm}$ isotropic); spatial smoothing using a Gaussian kernel of $4.0 \mathrm{~mm}$ full-width at half-maximum (FWHM); grand-mean intensity normalisation; and high-pass temporal filtering at $100 \mathrm{~s}(0.01 \mathrm{~Hz})$.

Then, using the FSL MELODIC tool, a temporally concatenated probabilistic ICA was applied. Briefly, we temporally concatenate all preprocessed fMRI data from all participants into a single new 4D 'group' fMRI series. To obtain the intrinsic brain components (RSNs) we applied the following steps. We started with the voxel-wise demeaning and variance normalisation. Subsequently, the pre-processed data were projected into a 34-dimentional subspace using principal component analysis. Then these observations were decomposed into sets of vectors which describe signal variations across the temporal domain (time-courses), the session/subject domain and the spatial domain (maps) by optimising for non-Gaussian spatial source distributions using a fixed-point iteration technique. The resulting estimated component maps were divided by the standard deviation of the residual noise and thresholded at a posteriori probability threshold of $\mathrm{p}>.5$ (i.e., an equal loss is placed on false positives and false negatives) by fitting a Gaussian/gamma mixture model to the histogram of intensity values (Beckmann and Smith, 2004). The number of components was arbitrarily set to 34 as it seems to be a good trade-off to get a sufficient number of relevant networks (around ten), without splitting them into subcomponents (Wang et al., 2011). The most relevant RSNs were then selected, by first discarding component

\footnotetext{
$2_{\text {ww. fmrib.ox.ac.uk/fsl }}$
} 
with too much power (more than $1 / 3$ of the total power) in high-frequency range $(>0.1 \mathrm{~Hz})$ of their spectrum; and then using an in-house built 'goodness-of-fit' MatLab function upon the 10 RSN map templates from Smith(Smith et al., 2009a). Briefly, we calculate the difference between the average Z-scores of the voxels (of our group RSNs) falling within the template network and the average Z-score falling outside the template network (Greicius et al., 2004; Vanhaudenhuyse et al., 2010). The best 'fit' network is represented by the highest difference (score). Also as final step, we visually check and validate the network. This final step can also lead to selecting other network that are not in Smith's template, but passes the first (power) selection criterion (2/3 of power under $0.1 \mathrm{~Hz}$ ), while not describing noise or artefacts.

Finally, a dual regression is used upon these selected group RSNs in order to extract subjectspecific RSN maps and their associated time series (Beckmann et al., 2009). Briefly, we extract first subject-specific time series by regressing out the group RSN maps from the individual raw fMRI. Then we regress those time series out of the individual raw fMRI, giving us the subjectspecific RSN spatial map. Here, we use that second output: subject-specific maps, to test whether or not groups differ in spatial connectivity (within RSN). And we use the first regression output: subject-specific time series (of the RSNs) for our wavelet coherence analysis described in the next subsection. This procedure of preprocessing the data and extracting subject-specific maps and time series was performed for both the in-house and Leuven datasets (independently). Prior to the wavelet coherence analysis presented in the next section, it is worth noticing that a groupwise comparison of each of the RSNs was applied on the subject-specific spatial RSN maps, using Threshold Free Cluster Enhanced (TFCE) method, and a non-parametric 5000-permutation test (Smith and Nichols, 2009).

\subsubsection{Wavelet coherence and time of in-phase coherence}

Using the aforementioned selected RSN time series, wavelet coherence maps between pairs of brain networks can be drawn. More details of how wavelet coherence maps (or scalogram) are constructed can be found in the paper from (Torrence and Compo, 1998). Briefly, they define $R^{2}(t, s)$ as local correlation coefficients in time $(t)$ and wavelet scale $(s)$, between two signals $\mathrm{X}$ and Y. In order to get the coefficients, a wavelet cross-spectrum between the two signals is applied, measuring the common power between the signals at various scales (s) and time ( $t$ ). Also, the phase difference between $\mathrm{X}$ and $\mathrm{Y}$ is can be calculated with $\arg \left(R^{2}(t, s)\right)$. When applied on pairs of RSN time series, subject-specific wavelet coherence scalograms are extracted. Figure 5.2 shows an example of two wavelet transforms, and the derived wavelet coherence scalograms (or maps) from an ASD patient.

By combining the wavelet coherence coefficients $R^{2}(s, t)$ and their phase information $\arg \left(R^{2}(t, s)\right)$, we measured the average of time of in-phase coherence (Bernas, 2016), per wavelet scale (Fourier period) as

$$
c(s)=\frac{100}{N} \sum_{t=1}^{N} \mathbb{I}\left\{R^{2}(t, s)>a_{95}\right\} . \mathbb{I}\left\{-\frac{\pi}{4}\left\langle\arg \left(R^{2}(t, s)\right)\right\rangle \frac{\pi}{4}\right\},
$$

which can be seen as the level/density of coherent synchronicity between two RSNs over time. $N$ represents the number of time points (fMRI volumes); $\mathbb{I}\{$.$\} is 1$ or 0 whether or not the conditions between brackets is true (here significance and in-phase conditions); and $a_{95}$ is the coherence coefficient threshold above which the wavelet-coherence coefficients are considered significant. Finally, to determine this threshold, the significance of the coherence coefficients (delineated areas in Figure 5.2) was tested against wavelet coherence of random red noise signals using Monte Carlo methods with 1000 surrogate data set pairs (Grinsted, Moore, and Jevrejeva, 2004). Briefly, 
we apply the wavelet coherence upon 1000 random signal pairs (red noise) with similar 1-lag autoregressive (AR1) coefficients as our RSN time series pair. Thus, we get a null distribution of wavelet coherence coefficients, which we used to define the $5 \%$ significance level, i.e., to threshold (here drawn as a contour) of our wavelet coherence coefficient maps.

Wavelet coherence maps, or scalograms, were calculated using the method explained above and the toolbox ${ }^{3}$ from Grinsted, Moore, and Jevrejeva, 2004. Also, we used the complex Morlet wavelet as it has the best ratio $(=1.03)$ between Fourier period and wavelet scale which helps interpreting results in the frequency domain (Torrence and Compo, 1998). Also, we used the complex Morlet wavelet as it has the best ratio $(=1.03)$ between Fourier period and wavelet scale which helps interpreting results in the frequency domain. Finally, these maps were composed with 12 subscales per octave-an octave being the range of periods between $2^{\mathrm{n}}$ and $2^{\mathrm{n}+1} \mathrm{~s}$, with $n$ an integer between 2 and 6 . The seventh octave $(n=7)(128-256 \mathrm{~s})$ being incomplete and under $0.01 \mathrm{~Hz}$ we did not test them because of our high-pass filtering cut-off frequency. For the first octave (2-4 s), the frequencies are greater than the Nyquist frequencies $(0.25 \mathrm{~Hz}$ and $0.31 \mathrm{~Hz}$ for in-house and Leuven datasets respectively). Therefore we did not test them either. Finally, the sixty subscales (12 sub-octaves in 5 octaves) described above represent the rows of the wavelet coherence maps (Figure 5.2).

\subsubsection{Classification}

After extracting the time of in-phase coherence between pairs of RSNs, we trained classifiers and compared the performance results of several classifications methods. In this study, Linear Discriminant Analysis (LDA), and Support Vector Machine (SVM) were used. Two kernels for SVM classifiers were tested: a polynomial (Poly-SVM) and a Radial basis function (Rbf-SVM). All these classifiers were tested using our in-phase coherence features extracted at each of the suboctaves. Using these features we train and validate independently each aforementioned classifier type (LDA and SVMs). The feature vectors in this study are the dynamics, in term of time of in-phase coherence, between pairs of RSNs. At final stage, two setups for the feature vector are constructed: using 4 features, i.e., the time of in-phase coherence from 4 RSNs pairs; and 2 features (from 2 RSNs pairs). Those 2 or 4 pairs are compound with networks involving auditory, visual, fronto-parietal (right and left) and the attention ventral stream (more details for the choice of the networks for the 2 setups in the Results section). In order to validate the classifiers, we used the leave-one- out cross-validation technique upon each of the sample (in-house and Leuven). This leads to a 24- and 30-folds LOOCV, for the in- house and Leuven data respectively. Finally, we trained our classifiers on the in-house dataset and tested them on the Leuven data. The classification quality is measured in terms of sensitivity (proportion of (true) ASD correctly classified), specificity (proportion of the (true) controls correctly classified), positive predictive value or precision (PPV, proportion of the predicted ASDs correctly classified), and negative predictive value (NPV, proportion of the predicted controls correctly classified) 


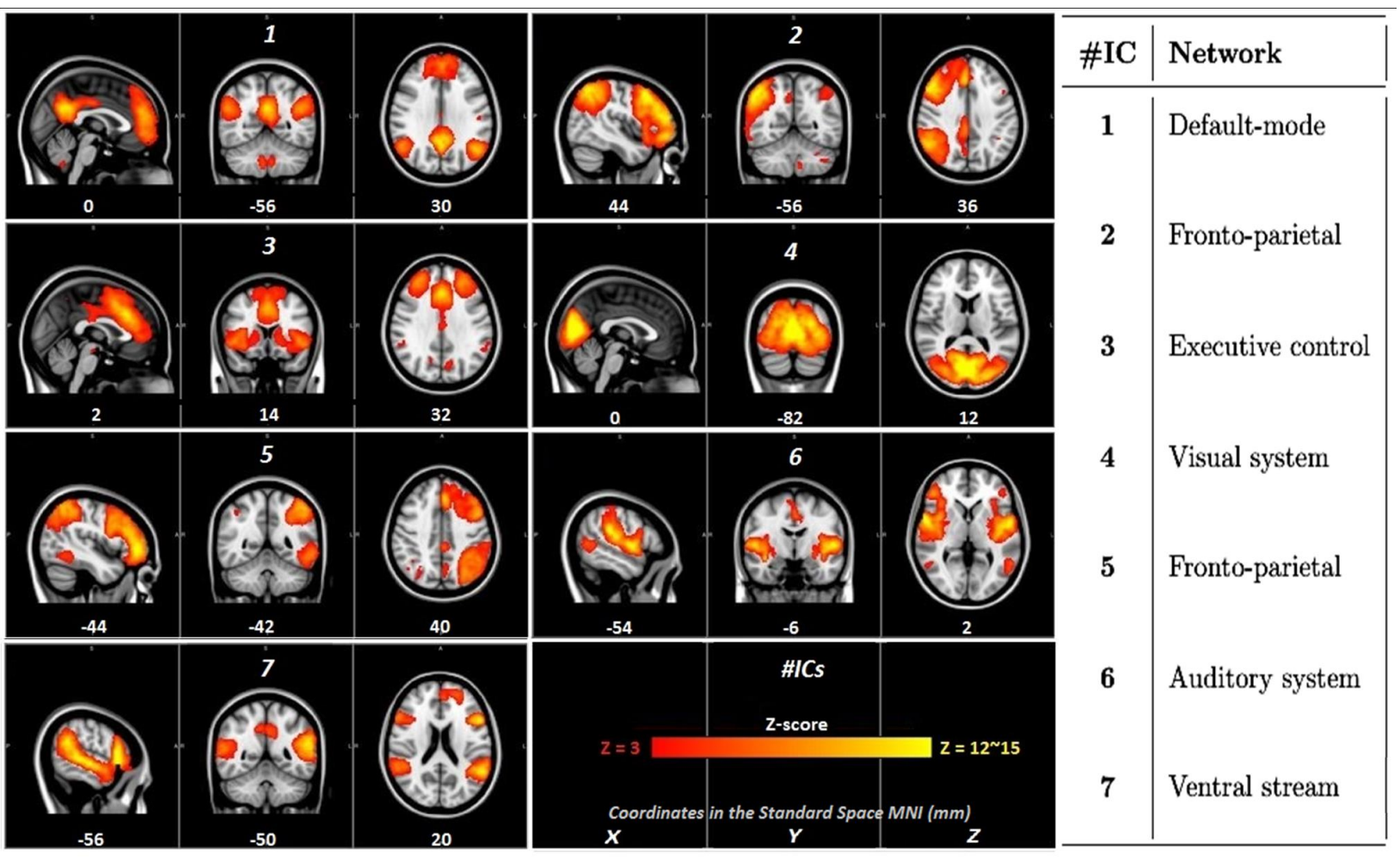

FIGURE 5.1: The seven most relevant networks identified in our in-house sample for both groups (ASD and normal controls).

\subsection{Result}

\subsubsection{Resting-state network extraction}

After preprocessing the data and applying gICA upon our in-house sample, eleven networks that most optimally matched the template of (Smith et al., 2009a) were selected. For further analysis, we focused on the seven fronto-parietal and temporal networks, typically involved in the executive and socio-cognitive functions. The seven networks, depicted in Figure 1, encompass the ventral stream (VENT) network, the central-executive network (EXE), the fronto-parietal left and right (respectively FPL, FPR), the auditory system (AUDI), the visual cortex (VISU) and the default-mode network (DMN). Except for VENT, all the aforementioned RSNs are described in (Smith et al., 2009a). VENT is composed of lateral visual, superior temporal sulci, frontal poles and opercula cortices. It also overlaps with executive networks (EXE, FPL, FPR) in the medial superior frontal gyrus, and with the sensorimotor network through the supplementary motor area. This network configuration can be seen as a combination of well-known networks: the attentionalventral, sensorimotor-ventral and salient networks (Choe et al., 2015; Menon and Uddin, 2010). Next, in the Leuven dataset, the same seven networks were extracted using the same method.

When comparing each of these relevant networks, using TFCE and the permutation test implemented in FSL, none of them showed spatial differences between controls and ASD, i.e., in average controls and ASD adolescents have the same functional networks. This was replicated in the Leuven dataset, and showed no differences in spatial RSNs maps between ASD and controls.

\footnotetext{
${ }^{3}$ http://www.glaciology.net/wavelet-coherence
} 


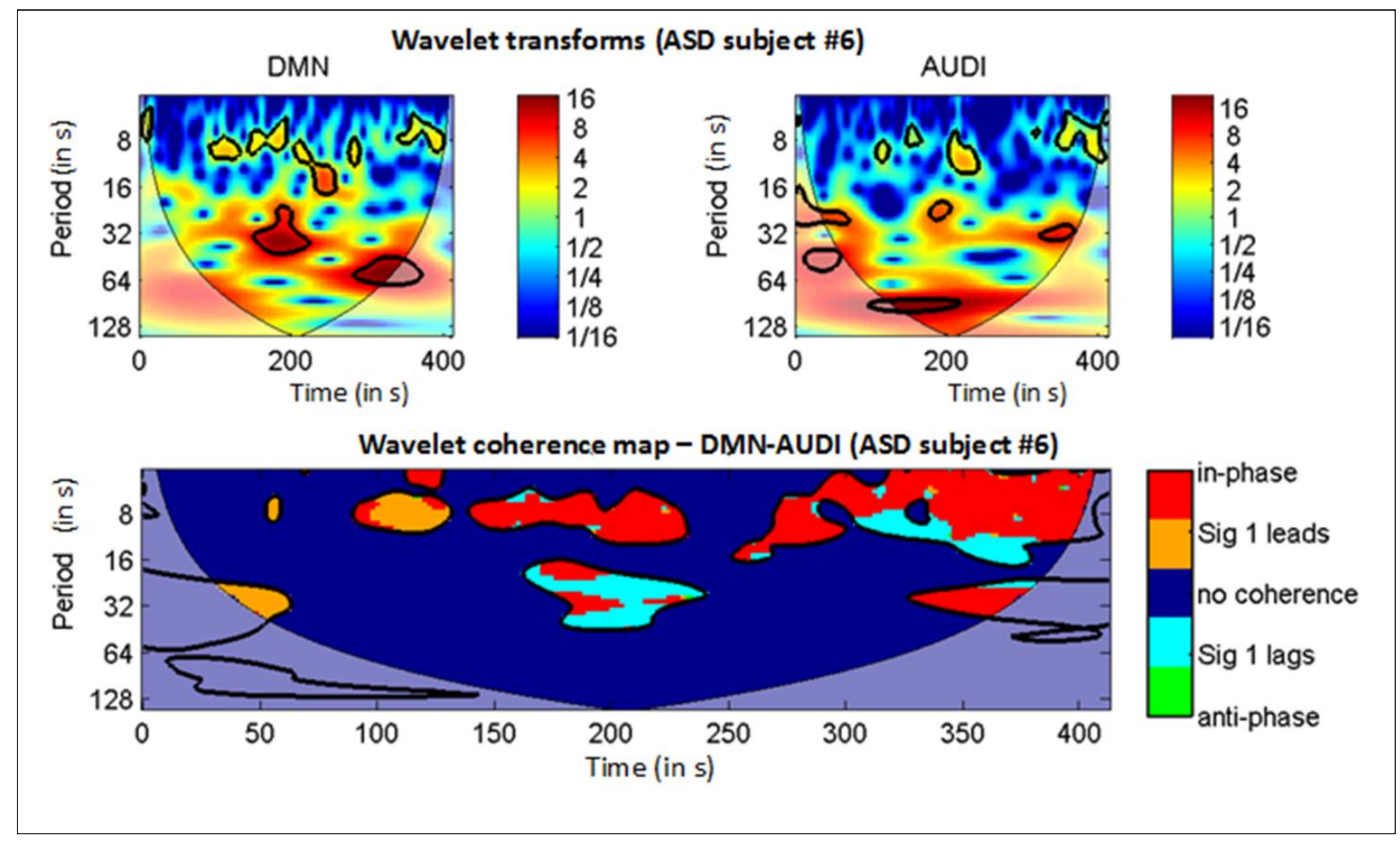

FIGURE 5.2: Continuous wavelet transform upon two resting-state signals: default mode network (top-left scalogram) and auditory system (top-right scalogram), and their wavelet coherence (bottom scalogram) for the $6^{\text {th }}$ ASD subject of our in-house dataset. In the wavelet coherence scalogram, delineated and coloured area indicates significant coherence. colours within the areas determined the phase difference between the two networks (DMN and AUDI): signals are in phase (red), anti-phased (green); DMN leads AUDI (orange), and AUDI leads DMN (light blue). Outside the delineated area, there is no coherence (dark blue). For each scalogram, the x-axis indicates time (in seconds), and the y-axis, the scale (in Fourier periods).

\subsubsection{Wavelet coherence maps and time of in-phase coherence}

With the dual-regression method implemented in FSL (Beckmann et al., 2009), we extracted from the 7 RSNs (Figure 1) subject specific RSN time series. On these signals, a wavelet coherence analysis can be conducted, as explained in material and methods. This was done upon the 21 possible RSN time series pairs we could form. Figure 5.2 shows two wavelet transforms, and an example of the ensuing wavelet coherence map obtained between DMN and AUDI for one ASD participant from our in-house sample. Also, as we used a complex wavelet (Morlet), it provided us with phase information, allowing visualisation of directionality in the dynamics between signals (in-phase, leading, lagging, or anti-phase).

\subsubsection{Time of in-phase coherence}

We obtained, as shown in Figure 5.2, the wavelet coherence scalograms for each subject and for each pair of relevant RSN time series. The ratio of the red area over the full time length $(7 \mathrm{~min})$ of a row of these wavelet coherence scalograms, represents the time (in \%) of in-phase coherence for a specific period (the chosen row). Figure 5.3 shows a group-wise (ASD and controls) average of these times of in-phase coherence per scale (from $4 \mathrm{~s}$ to $128 \mathrm{~s}$ periods). For different pairwise combinations of the seven relevant networks (Figure 5.1), we can observe noticeable differences between the groups in Figure 5.3. For instance, in the periods of $8 \mathrm{~s}$ to $16 \mathrm{~s}$ for the pair VENT-FPR, a clear difference between the groups is visible (Figure 5.3 , top graphs), whereas we observe less 


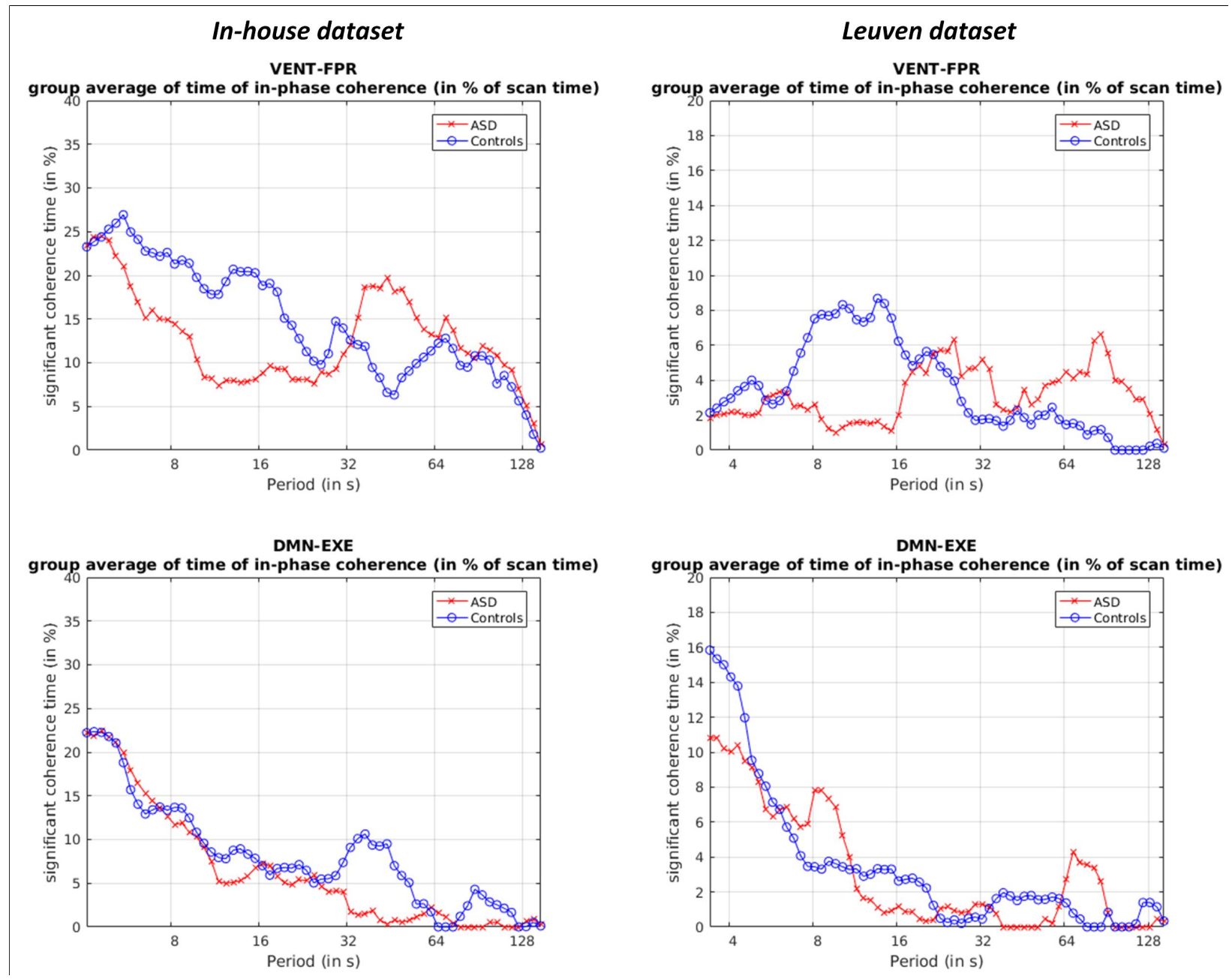

FIGURE 5.3: Average of time of in-phase coherence per bin (12 scales per octave) in ASD (red) and controls (blue) for the pairs of networks VENT-FPR (top), or DMN-EXE (bottom). Left - graphs for the in-house dataset, right - results for the Leuven dataset

variability between groups in the pairs DMN-EXE. The pairs of networks where such differences between the groups were visible were intuitively good features to test our classifiers upon.

\subsubsection{Classification and performance}

Using the aforementioned wavelet coherence maps (e.g., Figure 5.2) of pairs of networks, the times (in \%) of in phase coherence per scale and subject can be extracted. These measurements were then used as features for the classifiers. We used two to five pairs of network and their time of in-phase coherence to feed three types of classifiers: LDA, rbf-SVM and poly-SVM. To select the best pairs of socio-executive networks, we inspected the average per group of time of in-phase coherence of all pairwise combination of the seven relevant networks (example in Figure 5.3). Interestingly, most of the pairs involving the VENT network showed a clear discrepancy in the wavelet octave $8 \mathrm{~s}$ to $16 \mathrm{~s}$. By training our classifiers with the time of in-phase coherence of the pairs VENT-AUDI, VENT-VISU, VENT-FPL, and VENT-FPR, we obtain the best results for our in-house datasets (using leave-one-out cross-validation): $87.5 \%$ accuracy (91.7\% sensitivity, 83.3\% specificity). However, this classifier was built at $6 \mathrm{~s}$ period for the wavelet scale, which is outside the frequency range of resting-state brain oscillation (from 0.01 to $0.1 \mathrm{~Hz}$ ) (Biswal et al., 1995). 
TABLE 5.2: Best classifier performances for ASD detection.

\begin{tabular}{|c|c|c|c|c|c|c|}
\hline Periods ${ }^{*}$ & Type & Accuracy & Sensitiviy & Specificity & PPV & NPV \\
\hline \multicolumn{7}{|c|}{$\begin{array}{l}\text { Features based on VENT-AUDI, VENT-FPL, VENT-FPR, VENT-VISU } \\
\text { In-house set leave-one-out cross-validation (24-fold) }\end{array}$} \\
\hline $6 \mathrm{~s}$ & LDA & 87.5 & 91.7 & 83.3 & 84.6 & 90.9 \\
\hline $5 \mathrm{~s}$ & Rbf-SVM & 70.8 & 66.7 & 75.0 & 72.7 & 69.2 \\
\hline $5.5 \mathrm{~s}$ & Poly-SVM & 66.7 & 75.0 & 58.3 & 64.3 & 70.0 \\
\hline \multicolumn{7}{|c|}{ Leuven dataset leave-one-out cross-validation (30-fold) } \\
\hline $11 \mathrm{~s}$ & LDA & 73.3 & 66.7 & 77.8 & 66.7 & 77.8 \\
\hline $10 \mathrm{~s}$ & Rbf-SVM & 76.7 & 75.0 & 77.8 & 69.2 & 82.4 \\
\hline $10 \mathrm{~s}$ & Poly-SVM & 86.7 & 83.3 & 88.9 & 83.3 & 88.9 \\
\hline \multicolumn{7}{|c|}{ In-house dataset for training with Leuven dataset for validation } \\
\hline $15 \mathrm{~s}$ & LDA & 66.7 & 100 & 44.4 & 54.6 & 100 \\
\hline $10 \mathrm{~s}$ & Rbf-SVM & 66.7 & 91.7 & 50.0 & 55.0 & 90.0 \\
\hline $11 \mathrm{~s}$ & Poly-SVM & 63.3 & 75 & 55.6 & 52.9 & 76.9 \\
\hline \multicolumn{7}{|c|}{$\begin{array}{l}\text { Features based on VENT-FPLR, VENT-FPR } \\
\text { In-house set leave-one-out cross-validation ( } 24 \text {-fold) }\end{array}$} \\
\hline $10.5 \mathrm{~s}$ & LDA & 75.0 & 83.3 & 66.7 & 71.4 & 80.0 \\
\hline $5.5 \mathrm{~s}$ & Rbf-SVM & 79.2 & 75.0 & 83.3 & 81.8 & 76.9 \\
\hline $9 \mathrm{~s}$ & Poly-SVM & 79.2 & 83.3 & 75.0 & 76.9 & 81.8 \\
\hline \multicolumn{7}{|c|}{ Leuven dataset leave-one-out cross-validation (30-fold) } \\
\hline $10 \mathrm{~s}$ & LDA & 83.3 & 91.7 & 77.8 & 73.3 & 93.3 \\
\hline $10 \mathrm{~s}$ & Rbf-SVM & 86.7 & 91.7 & 83.3 & 78.6 & 93.8 \\
\hline $10 \mathrm{~s}$ & Poly-SVM & 83.3 & 91.7 & 77.8 & 73.3 & 93.3 \\
\hline \multicolumn{7}{|c|}{ In-house dataset for training with Leuven dataset for validation } \\
\hline $10.5 \mathrm{~s}$ & LDA & 56.7 & 100 & 27.8 & 48.0 & 100 \\
\hline $13 \mathrm{~s}$ & Rbf-SVM & 66.7 & 91.7 & 50.0 & 55.0 & 90.0 \\
\hline $11 \mathrm{~s}$ & Poly-SVM & 80.0 & 100 & 66.7 & 66.7 & 100 \\
\hline
\end{tabular}

${ }^{\overline{ }}$ Fourier periods at which the classifier has been tested (the frequency of the in-phase coherence). More details in Appendix B (Table B.1- B.6).

Also, with an identical setup replicated with the Leuven dataset, the same LDA classifier scored at best at $73.3 \%$, and around 11 s periods.

In a second setup, we reduced the number of feature vector elements to two by removing the pairs linked to visual and auditory networks. This led to classifiers solely trained and validated with our dynamics features based on the pairs VENT-PFL and VENT-FPL. In that case, the results were more consistent: we obtained in both datasets the best results within the same range of frequency ( $9 \mathrm{~s}$ to $12 \mathrm{~s}$ ), and with the same classifier type (SVM). We obtained, in Leuven dataset at the range of $9 \mathrm{~s}$ to $12 \mathrm{~s}$ periods, a performance peak (at $10 \mathrm{~s}$ ) of $86.7 \%$ ( $91.7 \%$ sensitivity, $83.3 \%$ specificity). However, in this setup, our in-house classifiers performed less accurately than in the first setup with 4 feature vector elements (peak performance of $79.2 \%$ when tested with SVM, around $9 \mathrm{~s}$ periods).

Finally, using still the second setup, we could distinguish ASD from non-ASD in Leuven participants at $80 \%$ accuracy (with 100\% sensitivity, 66.7\% specificity), using a poly-SVM classifier trained with our in-house dataset. For both setups mentioned above, a summary of the best classification performances, for each classifier, and along with the periods at which the featured were extracted, is depicted in Table 5.2. More performance details at each subscale (periods) are available in Appendix B (Tables B.1- B.6). 

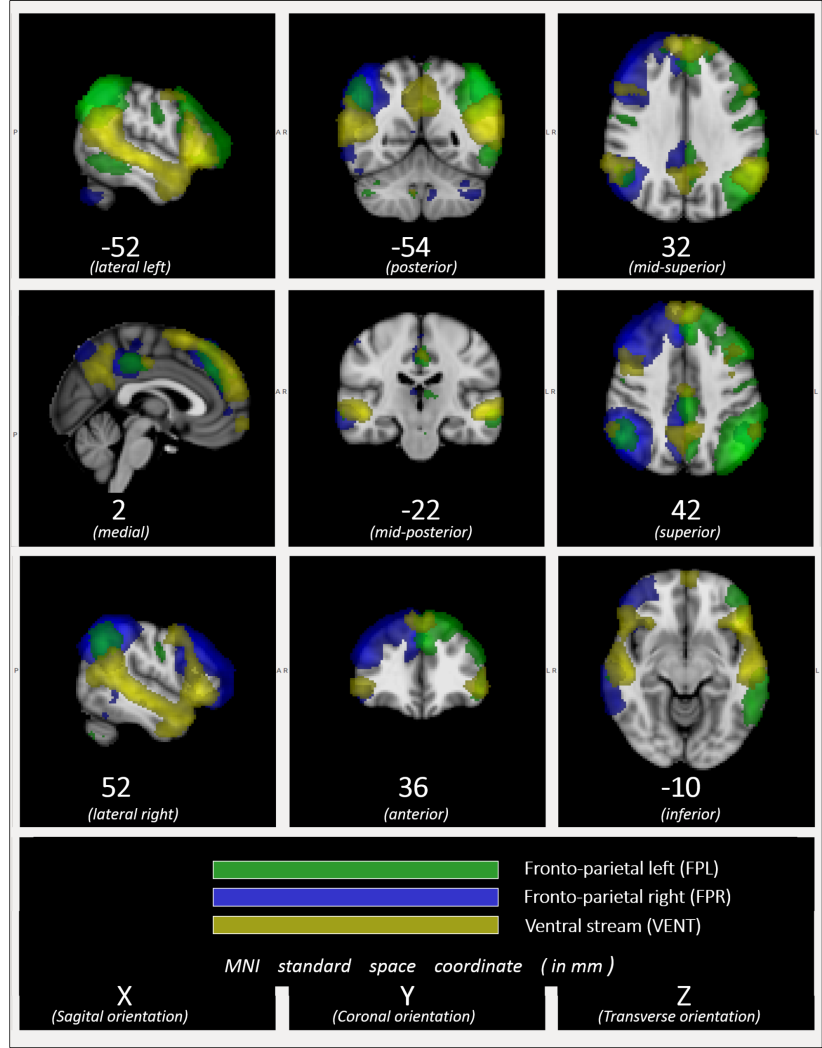

FIGURE 5.4: Medial and bilateral (left and right) visualisation of the group-wise RSNs (Leuven dataset): ventral stream (in yellow), and fronto-parietal left and right (respectively green and blue).

\subsection{Discussion}

Many studies have shown changed functional networks in children with ASD as compared to normal controls. Under- or hyper-connectivity within and between different RSNs is reported. The networks usually involved in such studies are the executive networks (EXE, or FPL, FPR) showing correlation with autistic behaviour (restrictive). Also the default mode and other socioemotional networks (involving limbic cortices) were identified, correlating generally with social ability impairments. However, results are contradictory and dependent on the scan protocol, age of the patient and other factors. In our study, for both datasets we actually did not find any statistically significant differences within relevant functional RSN between autism and control adolescents. This can be explained by the age range of the participants. Indeed, Nomi and Uddin demonstrated that, unlike children, within-network functional connectivity in adolescents with ASD do not differ from controls, but the between-network connectivity decreases in ASD (Nomi and Uddin, 2015). This is in line with our current results of between-network synchronicity being weaker in ASD, and our previous findings where we did not find within-network differences (Bernas, 2016).

However, more than their topology, temporal dynamics of functional network is a key feature in ASD (Chen et al., 2016; Hanson et al., 2013; Kana et al., 2014; Wicker et al., 2008). In our study, we focused on temporal brain dynamics between RSNs, using wavelet coherence maps. We found that these subject-specific maps can display pattern of synchronicity between brain networks over time, at different scales (or frequency ranges). Considering socio-executive networks, we detected group-wise differences, especially in the coherence between VENT-FPR and VENT-FPL. Using these two features in a machine learning algorithm, we could actually reach $86.7 \%$ accuracy of classifying ASD versus controls in both datasets. Also, we reached $80 \%$ accuracy while training 
our classifier on one dataset and testing it on other independent data. Our results are based on time of in-phase coherence. The anti-phased coherence could also be a strategy for further research. For example by involving EXE and DMN, that are usually functionally anti-correlated, an insightful metric to test our classifier could be developed. In the study of Yerys et al., 2015, the ASD groups demonstrated under-connectivity within the DMN, and a poorer network segregation of its midline core functional connectivity, as compared with controls. This midline core of the DMN encompassed the medial prefrontal cortex and posterior cingulate cortex. Even though testing our classifier upon pairs of networks including the DMN was not successful, the DMN midline core areas described in Yerys et al., 2015 are actually the overlapping parts of our three networks VENT, FPL, FPR (see Figure 5.4).

The fact that the best and consistent performance scores are obtained using only VENT-FPR and VENT-FPL is understandable. Indeed, the ventral stream encompassed the ventral part of the more frequently mentioned attentional network and partially (insular nodes) the salient network. Those two networks have been shown to have an important role in attention, and the dynamic switch between attention and executive control processes (Menon and Uddin, 2010; Uddin et al., 2011). Interestingly, the FPL and FPR involve language-related cognitive functions and perception-somesthetic functions, which are functions that can be impaired in ASD.

Moreover, the pairs VENT-FPL and VENT-FPR show ipsilateral and contralateral connectivity. Hence, our results suggest impairment in the temporal dynamics of the inter- or intra-hemispheric functional connectivity. In Lee et al. (2016), abnormalities were found in the functional connectivity density of both ipsilateral and contralateral part of the brain (Lee et al., 2016). These decreases in functional connectivity density (FDC) were located in the cingulate cortex, lingual/parahippocampal and postcentral gyrus cluster. More specifically, they found that the contralateral FCD of the posterior cingulate cortex and the precuneus correlated negatively with restrictive and repetitive behaviour scores (ADOS-RRB). These two cortices represent, again, our main overlapping areas in the parietal cortex.

Our results are also in line with recent findings involving autism and temporo-parietal junctions. Indeed, Igelstrüm and his colleagues demonstrated that these junctions and their connectivity with the cerebellum were crucial in social cognition (Igelström, Webb, and Graziano, 2016). We did not try combination of pairs of networks with the cerebellum to test our classifiers upon, but it is clear that parts of the cerebellum are contralaterally and functionally connected to the FPL and FPR (Figure 5.4) Also, these temporo-parietal junctions are mainly composed of our ventral stream.

To summarise, the aforementioned studies informed us about three findings: contralateral connections are weaker in ASD, especially in the regions that overlap and are inside the FPL, FPR, and VENT, as identified in our study; temporo-parietal connectivity plays a crucial role in social ability; and the ventral stream is usually seen as a dynamic switch between socio-executive functional networks. Moreover, when including the dynamics feature of the direct pair FPL-FPR into our classifiers, we did not obtain successful results (accuracy under 67\%). Hence, our capacity to classify autism using only the pairs VENT-FPL and VENT-FPR can be explained by a less coherent fronto-parietal synchronicity, mediated by the ventral stream, and is associated with the deficit in language processes or the restrictive behaviour observed in ASD.

For a more technical point of view, we can see that poly-SVM is leading to the most stable results, providing, for all classifier tested around the periods 9-12 s, an average of classification accuracy greater than $75 \%$. Hence, the combinations of the dynamics (for 2 or 4 pairs) are non-linear, as compared to the LDA, or rbf- SVM. This also emphasised the non-linearity of brain connectivity (at least in terms of dynamics). 
And, as already mentioned in results, the periods at which coherences seem to differ between the group (ASD and controls) and in both datasets (in-house and Leuven) are around $10 \mathrm{~s}$. By smoothing the features through 3 subscales (i.e. averaging features over quarter-octave ranges of periods) we did not get better results, but the tendency of high accuracy around the central periods of $10 \mathrm{~s}$ still holds. However, testing at larger ranges (e.g., half-octaves smoothing) led to less accurate results.

We would like to point out the main limitation of the methods presented in this study: We cannot ensure similar diagnostic performances in an infant group with autism. To test whether or not the pairs of networks and their coherent dynamics used in this study provides similar results in young children, one can do 2 things: i) replicate the analyses with a large group (including children) and check if the features are age independent; ii) or use a stratified approach (by range of ages) and replicated the analyses, i.e., try to refine our current model. But this classification approach was the first step testing validity of our MRI methods and homogeneous group with confirmed diagnostic certainty is exactly suitable for that. But the subsequent studies have to test the value in younger and more heterogeneous groups. Also, the issue of maturation affect remains unsolved in our present study.

Finally, not only ASD can benefit from our approach for computer-aided diagnosis. Our wavelet coherence approach that we coined as neurodynamics is likely to be suitable for a wider range of neuropsychiatric disorders, such as ADHD, schizophrenia, bipolar disorders, and perhaps dementia. So far, promising results for disorder classification were only obtained for schizophrenia, and the proposed method is also based on brain dynamics measurements (Damaraju et al., 2014). In fact, our computer-aided diagnosis approach using brain dynamics metrics and fMRI opens a path for further research in psychoradiology.

\subsection{Conclusion}

We showed that wavelet-based coherence maps can be used to visualise coherent patterns (or time-synchronicity) between large-scale resting-state networks. Also, the extracted times of inphase coherence between socio-executive networks were especially good descriptors for ASD, and present themselves as a potential biomarker or at least can be used to as an additional tool for ASD diagnosis. The obtained wavelet time-synchronicity characteristics in ASD may reflect a less coherent fronto-parietal synchronicity, mediated by the ventral stream. These findings suggest the importance of assessing not only functional connectivity, but also brain temporal dynamics, in order to better describe a developmental disorder such as ASD. 
Chapter 6

Emulative, coherent, and causal dynamics between large-scale brain networks are neurobiomarkers of accelerated cognitive ageing in epilepsy

[Submitted] 


\begin{abstract}
Accelerated cognitive ageing (ACA) is an ageing co-morbidity in epilepsy that is diagnosed through the observation of an evident IQ decline of more than 1 standard deviation (15 points) around the age of 50 years old. To understand the mechanism of action of this pathology, we assessed brain dynamics with the use of resting-state fMRI data.

In this paper, we present novel and promising methods to extract brain dynamics between largescale resting-state networks: the emulative power, wavelet coherence, and granger causality between the networks were extracted in two resting-state sessions of 24 participants (10 ACA, 14 controls). To find the best biomarkers of ACA, and have a better understanding of this epilepsy co-morbidity we compared the aforementioned between-network neurodynamics using classifiers and known machine learning algorithms; and assessed their performance.
\end{abstract}

Results show that features based on the evolutionary game theory on networks (EGN) approach - the emulative powers-are the best descriptors of the co-morbidity, using dynamics associated with the default mode network, and the dorsal attention network. With these dynamic markers, linear discriminant analysis could identify ACA at $82.9 \%$ accuracy. Using wavelet coherence (WCoh) features with decision-tree algorithm, and static functional connectivity (sFC) features with support vector machine, ACA could be identified at $77.1 \%$ and $77.9 \%$ accuracy respectively. Granger causality (GC) fell short of being a relevant biomarker with best classifiers having an average accuracy of $67.9 \%$. Combining EGN-, WCoh-, GC-, and sFC-based features increased the classification performance up to $90.0 \%$ average accuracy using SVM, with a peak accuracy of $95.8 \%$.

The dynamics of the networks that lead to the best classifier performances are known to be challenged in elderly. Since our groups were age-matched, the results are in line with the idea of ACA patients having an accelerated cognitive decline. This classification pipeline is promising and could help diagnosing other neuropsychiatric disorders, and contribute to the field of psychoradiology. 


\subsection{Introduction}

Cognitive impairment is a common comorbidity in epilepsy. It is estimated that $65 \%$ of all patients show impairment in cognition, accounting for about half of the burden of disease (Helmstaedter et al., 2014). Using fMRI, research has mainly focused on specific cognitive impairments, and correlated them with aberrant functional connectivity in brain areas involved in focal epilepsy. For instance, in rolandic epilepsy, language and motor-related functions are impaired, and were linked to a reduced functional connectivity between sensorimotor network and Broca's area (Besseling et al., 2013). Also already at young age, children with frontal lobe epilepsy complicated by cognitive impairment (memory) showed decrease in frontal lobe connectivity (Braakman et al., 2013). In an elderly population, the decline in cognitive abilities is also clearly present, yet often neglected (Hermann et al., 2008). This more general cognitive decline is often due to long-term accumulating effects, i.e. early onset and chronic epilepsy, and heavy use of anti-epileptic drugs, which hinders healthy mental ageing (Helmstaedter and Elger, 2009). This is often described as a chronic/accumulative model of cognitive decline, and viewed as a form of dementia (Sen, Capelli, and Husain, 2018). More recently, another model of cognitive decline in elderly has been presented. It is different from the chronic decline since it relates to epilepsy that has a late onset, where the decline is more abrupt and occurs soon after the diagnosis of epilepsy. Also, it does not seem to degrade the crystallized cognitive functions such as long-term memory and language abilities; but rather the fluid intelligence: perceptual reasoning and processing speed. This fast mental ageing is coined 'accelerated cognitive ageing', or ACA (Breuer et al., 2016a; Breuer et al., 2019). Even though the clinical characteristics of the ACA comorbidity has been described, the brain mechanism of action of the pathology remains unclear. This is why in this study we assess, using fMRI, different dynamic parameters of large-scale networks that could help describing neuronal mechanisms behind the decline.

The brain has been proven to efficiently work in functionally connected networks, i.e. the aggregation of multiple region of interests (ROIs; Heuvel and Hulshoff Pol, 2010; Power et al., 2011). Also, the links between brain activity and behaviour/cognition are often better analysed through the use of large-scale networks (Cole, 2010; Laird et al., 2011; Nomi and Uddin, 2015; Smith et al., 2009b). Therefore, the resting-state network (RSN) dynamics are informative features in neuropsychiatric disorders and can be used as neurobiomarkers. Between-network neurodynamics analysis can be performed using network time series from a spatial independent component analysis (ICA), a reliable data-driven method (Beckmann et al., 2005; Choe et al., 2015). In a seed-based approach (voxel-to-voxel, or ROI-to-ROI), the seeds or ROIs have to be defined a priori, and this can be quite inter-subject variable, especially for resting-state (task-free) fMRI. Hence the choice of ICA decomposition for extracting the functional brain RSNs and their activity time courses is favourable for robust rs-fMRI group analysis, and is preferred here.

After extracting the time series per network, diverse methods can be applied to estimate the dynamics of these networks. Currently, to find the abnormalities in brain functioning of patients with neuropsychiatric disorders, research has mainly focused on static functional connectivity (sFC), i.e. pairwise correlations between the functional networks' activity. It has been shown that at rest the default-mode network is involved in the impairment in autism (Hull et al., 2017; Müller et al., 2011); and the attention and executive networks in attention deficit hyperactivity disorder (ADHD; Park, Kim, and Park, 2016; Zang et al., 2007). But for more complex mental illnesses (e.g. depression or schizophrenia) dynamic measures of the brain connectivity seem to be more robust biomarkers (Kaiser et al., 2016; Rashid et al., 2014).

In that regard, effective connectivity (EC) can be used (Friston, Moran, and Seth, 2013; Friston, 2011). The EC, or measure of causality in brain connectivity, has already shown promising results for autism and depression (Bernas et al., 2018; Rolls et al., 2018). However, in terms of cognitive 
decline in the ageing process, clear causal dynamics indicators are missing. Liu et al. have shown links between cognitive decline and changes in Granger causality (a measure of EC) of cognitive networks in mild cognitive impairment (Zhenyu Liu et al., 2012). Using a different-more hypothesis driven-causal measurement, the dynamic causal modeling (DCM), Tsvetanov et al. showed that differences in between and within large-scale resting-state networks were linked to cognitive performance in elderly (Tsvetanov et al., 2016). More precisely they demonstrated that the DCM of the default mode, dorsal attention and salience networks (DMN, DAN, SN) where associates with increased influence on cognitive functioning - that tends to decline-in healthy elderly. Another study showed the involvement of DMN and SN in the cognitive decline in cognitively impaired elderly using also the DCM approach (Chand et al., 2017).

More recently, to assess neuro- and psycho-pathology, the dynamic functional connectivity (dFC) methods have been used as well (Calhoun et al., 2014; Damaraju et al., 2014; Di and Biswal, 2013; Kaiser et al., 2016; Li et al., 2019; Liégeois et al., 2019). These are similar to the sFC but when correlation coefficients are calculated over different time windows. By sliding these windows throughout the full time series, one can extract the dFC between the networks being assessed (Hindriks et al., 2016; Shakil, Lee, and Keilholz, 2016). This method of analysis has been for example applied to extract abnormal brain states in the study of schizophrenia, which has stronger classification power as compared to classic static functional connectivity (Rashid et al., 2016). The same methods can also be applied in the frequency domain, and the sliding windows approach can be applied on Fourier spectra, giving rise to spectrograms. And similar spectro-temporal analysis of wavelet coherence has not been much used in fMRI studies. Chang and Glover have shown added value in assessing non-stationarity and time-varying anti-correlation between DMN and cognitive networks, using wavelet coherence (Chang and Glover, 2010). To relate to cognitive decline, only one study used wavelet coherence analysis of near-infrared spectroscopic signals to infer changes in brain functional connectivity in respect to performance in vigilance task, suggesting that decreased attention-a known phenomenon in ageing, and probably associated to cognitive decline-was attributed to reduced phase coherence between left prefrontal region and sensorimotor area. In a previous rs-fMRI study, we have successfully shown that coherent patterns, using wavelet coherence, are also impaired in autism; and the duration of these coherent patterns can be used as biomarker of the disorder (Bernas, Aldenkamp, and Zinger, 2018).

Finally, evaluation of brain dynamics can be done through an evolutionary game theory on networks (EGN) approach. Madeo and his colleagues were first to implement the methods on BOLD fMRI signals (Madeo et al., 2017). They demonstrated that EGN model is able to mimic functional connectivity dynamics, which could then be used to simulate the impact of brain network lesions onto the networks' dynamics. This was done using simulated time series and real fMRI data from healthy subjects. Here, we present the first fMRI study where the EGN model is applied to a clinical population in order to see if network emulative powers (parameters of the model) can be descriptors of ACA.

The goal of our research is to define the most informative fMRI neurobiomarkers for ACA based on neurodynamics metrics. To achieve this, we use these metrics as features and train classifiers for ACA detection. The features that lead to the best classification results can then be considered the best descriptors of ACA. Therefore, in our study, the aforementioned neurodynamics techniques are used to extract fMRI-base dynamic features of brain networks, which are then fed to classifiers, to train and validate them. Decision tree, linear discriminant analysis, the K-nearest neighbors and the support vector machine are common supervised machine learning algorithms that divide the training data in two or more categories. Counting the number of correct binary classification, in the validation dataset, leads to a certain amount of accuracy, i.e. the percentage of participants that has been correctly assigned to their respective label (in our case, ACA or non-ACA/controls); which shows the descriptive power of the features, and to a certain extent 
its usability in a clinical environment. Convolutional neural network (CNN) can also be used to extract and test specific brain features using resting-state fMRI. It has already been tested in order to help diagnosing neuropsychiatric disorders, such as autism (Ktena et al., 2018), ADHD (Deshpande et al., 2015), and schizophrenia (Kim et al., 2016). An important drawback of this classification technique is that it usually needs a large amount of data to be trained upon; plus, the understanding and explanation of the feature maps obtained at different CNN layers can be arduous and troublesome. This is why we used here the aforementioned supervised machine learning algorithms and assessed the performance of classifiers using neurodynamics metrics, in order to find the best biomarkers of the ACA comorbidity, which if replicated could help describing the mechanism of action of the cognitive decline. Testing the classification pipeline, we describe here, in a bigger dataset stratified (e.g. through duration since onset of epilepsy, or age ranges) could even help predicting the course of the decline, and its severity.

\subsection{Materials and Methods}

Figure 6.1 provides an overview of the Materials and methods section, and short descriptions of the steps of the study pipeline. 


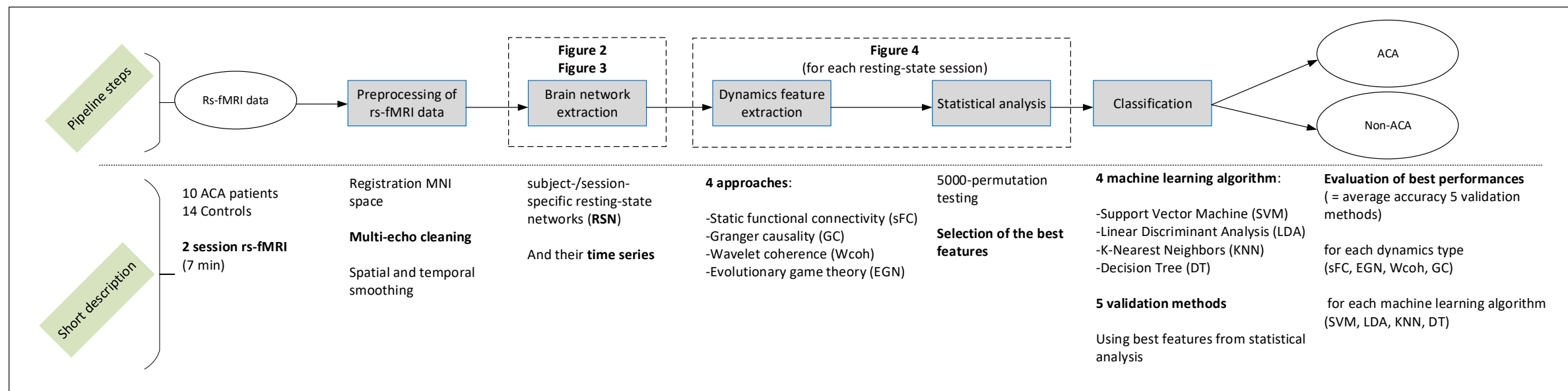

FIGURE 6.1: Overview of the methodology. 


\subsubsection{Dataset, preprocessing, and brain network extraction}

The dataset contains two resting-state (RS1 and RS2) sessions acquired from 10 ACA patients and 14 controls. Prior to RS2, a silent word generation task was performed for $7 \mathrm{~min}$. Dynamics features that show significant differences between the two populations in RS1 as well as in RS2, would show robustness of the features as biomarkers. Indeed, since the task in-between restingstates was cognitively demanding, such features would have discriminant power regardless of the brain fatigue the participants experienced. On the contrary, the difference in findings between RS1 and RS2 could shed light on the cognitive reserve capacity of the ACA patients, which can be of interest too. Table 6.1 shows the demographics of the participants, the type of epilepsy, and the deterioration scores (cognitive decline) for Full-Scale IQ, Perceptual Reasoning Index, and Verbal Comprehension Index (respectively FSIQ, PRI, and VCI). Deterioration scores were calculated by subtracting the estimated premorbid IQ scores from the actual scores, i.e. WAIS-IV (actual) IQscores - OPIE-IV (premorbid) IQ-scores (Holdnack et al., 2013; Wechsler, 2008).

The T1-weighted anatomical images were acquired using a $3.0 \mathrm{~T}$ imaging system (Philips Achieva) with a 3D Fast Field Echo (FFE) sequence: echotime $(\mathrm{TE})=3.8 \mathrm{~ms}$, repetition time $(\mathrm{TR})=8.4 \mathrm{~ms}$; Inversion time $(\mathrm{TI})=1035 \mathrm{~ms}$; field of view $(\mathrm{FOV})=240 \times 240 \mathrm{~mm} 2$, with 180 sagittal slices; flipangle $(\mathrm{FA})=8 \mathrm{deg}$; and voxel size $=1 \times 1 \times 1 \mathrm{~mm}^{3}$; with SENSE factor of 1.5 , and scan duration of 6:02 min. Functional MRI-data were acquired using multi-echo echo-planar imaging (ME-EPI) sequences with 3 echoes: TEs $=12,35,58 \mathrm{~ms}$, TR $=2$ s; FA $=90$ deg.; SENSE factor $=2.7 ; 208$ dynamics for a total of 7 minutes; 27 axial slices (with no gap), $64 \times 64$ matrix FOV, with a $3.5 \times 3.5 \times 4.5$ $\mathrm{mm}^{3}$ voxel size. The raw multi-echo fMRI data were first preprocessed following the pipeline of Kundu et al., 2012, using the python script meica.py ${ }^{1}$. Multi-echo- (ME)-ICA cleaning was applied in order to denoise each individual fMRI scan (Kundu et al., 2017). ME-ICA cleaning has been proven to be the most robust denoising method for resting-state fMRI, and tend to improve substantially effect sizes and statistical power (Dipasquale et al., 2017; Lombardo et al., 2016). Multi-echo cleaned data were further processed using FEAT (FMRI Expert Analysis Tool) Version 6.00, part of $\mathrm{FSL}^{2}$. The following pre-statistics processing was applied; spatial smoothing using a Gaussian kernel of FWHM 5.0mm; grand-mean intensity normalisation of the entire 4D dataset by a single multiplicative factor; high-pass temporal filtering (Gaussian-weighted least-squares straight line fitting, with sigma $=50.0 \mathrm{~s}$ ). The ME-ICA cleaning preprocessing led to an average (among all participant/scans) of 18 activity-related independent components (ICs or networks). Therefore, we chose 18 degrees of freedom for our group-ICA decomposition. The 18 spatial maps corresponding to functional resting-state networks (RSNs) were extracted using group-ICA followed by dual regression as implemented in FSL (Beckmann et al., 2009; Filippini et al., 2009). After the dual-regression steps, we obtained all subject/session-specific RSN maps and their associated time series. This brain network extraction step is depicted in Figure 6.2.

After visual inspection and a goodness-of-fit function implementation (Greicius et al., 2004), to match these maps with the RSN template from (Smith et al., 2009b), 4 noise-related RSNs were discarded, and 14 relevant functional brain networks were kept for further analyses. The time series associated with these 14 RSNs were then used to extract our brain dynamics features as explained in the next section. The 14 networks, or Independent Components (ICs), and their labelling are depicted in Figure 6.3.

\footnotetext{
$1_{\text {www.bitbucket.org/prantikk/me-ica }}$

2FMRIB' sSoftwareLibrary, www.fmrib.ox.ac.uk/fsl
} 
TABLE 6.1: Demographic, clinical and neuropsychological characteristics of the study participants

\begin{tabular}{|c|c|c|}
\hline & ACA & Healthy controls \\
\hline Age in years $-\mathrm{M}$ (std) [range] & $61.3(8.9)[50-74]$ & $62.2(9.8)[47-79]$ \\
\hline Gender & $70.0 \%$ male & $35.7 \%$ male \\
\hline Handedness & $100.0 \%$ right-handed & $92.9 \%$ right-handed \\
\hline Age at epilepsy onset & 35.0 (14.6) [15-59] & - \\
\hline Duration of epilepsy & $22.3(15.2)[1-50]$ & \\
\hline Type of epilepsy & $\begin{array}{l}40.0 \% \text { cryptogenic localized } \\
40 \% \text { sympomatic } \\
20 \% \text { idopathic }\end{array}$ & - \\
\hline Dominant seizure type $\mathrm{a}^{\mathrm{a}}$ & $\begin{array}{l}10.0 \% \text { simple partial } \\
20.0 \% \text { complex partial } \\
20 \% \text { absence } \\
50 \% \text { seizure free }\end{array}$ & - \\
\hline Status epilepticus & $50.0 \%$ yes & - \\
\hline Seizure frequency & $\begin{array}{l}50.0 \% \text { seizure }(\mathrm{sz}) \text { free } \\
0.0 \%<1 \mathrm{sz} / \mathrm{y} \\
20.0 \% 1-5 \mathrm{sz} / \mathrm{y} \\
20.0 \% 1 \mathrm{sz} \text { per } 2 \text { months } \\
0.0 \% \text { montly sz } \\
10.0 \% \text { weekly sz } \\
0.0 \% \text { daily sz }\end{array}$ & - \\
\hline Drug load ${ }^{\mathrm{b}}$ & $1.0(0.4)$ & - \\
\hline \multicolumn{3}{|l|}{ WAIS-IV indexes } \\
\hline FSIQ & $76.7(8.7)^{*}$ & $108.4(13.4)$ \\
\hline $\mathrm{VCI}$ & $94.7(10.6)$ & $106.2(12.2)$ \\
\hline PRI & $75.8(6.9)^{*}$ & $103.2(13.8)$ \\
\hline WMI & $79.6(10.0)^{*}$ & $104.9(13.3)$ \\
\hline PSI & $67.0(14.4)^{*}$ & $114.6(8.7)$ \\
\hline \multicolumn{3}{|l|}{ OPIE-IV scores } \\
\hline OPIE-IV FSIQ & $98.8(9.2)$ & $109.6(12.4)$ \\
\hline OPIE-IV VCI & $95.2(11.7)$ & $105.6(14.6)$ \\
\hline OPIE-IV PRI & $96.8(9.3)$ & $102.5(12.4)$ \\
\hline \multicolumn{3}{|l|}{ Deterioration scores $^{\mathrm{c}}$} \\
\hline DET FSIQ & $-22.1(5.0)^{*}$ & $1.3(8.2)$ \\
\hline DET VCI & $-0.5(8.9)$ & $0.2(6.0)$ \\
\hline DET PRI & $-21.0(4.5)^{*}$ & $0.0(8.7)$ \\
\hline \multicolumn{3}{|l|}{ Memory scores } \\
\hline Auditory & $93.9(9.7)^{*}$ & $109.9(12.6)$ \\
\hline Visual & $92.7(7.0)$ & $102.8(10.9)$ \\
\hline Delayed memory & $93.5(8.4)^{*}$ & $106.3(10.9)$ \\
\hline
\end{tabular}

${ }^{*} \mathrm{p}<.01$ significant difference between groups. WMI: Working Memoey Index. PSI" Processing Speed Index.

${ }^{a}$ Dominant seizure type is determined for the two years preceding neuropsychological assessment

${ }^{b}$ The prescribed daily dose of antiepileptic medication divided by the define daily dose (Lammers et al., 1995)

${ }^{\mathrm{c}}$ The deterioration scores = [WAIS-IV (actual) IQ-scores - OPIE-IV (premorbid) IQ-scores] 


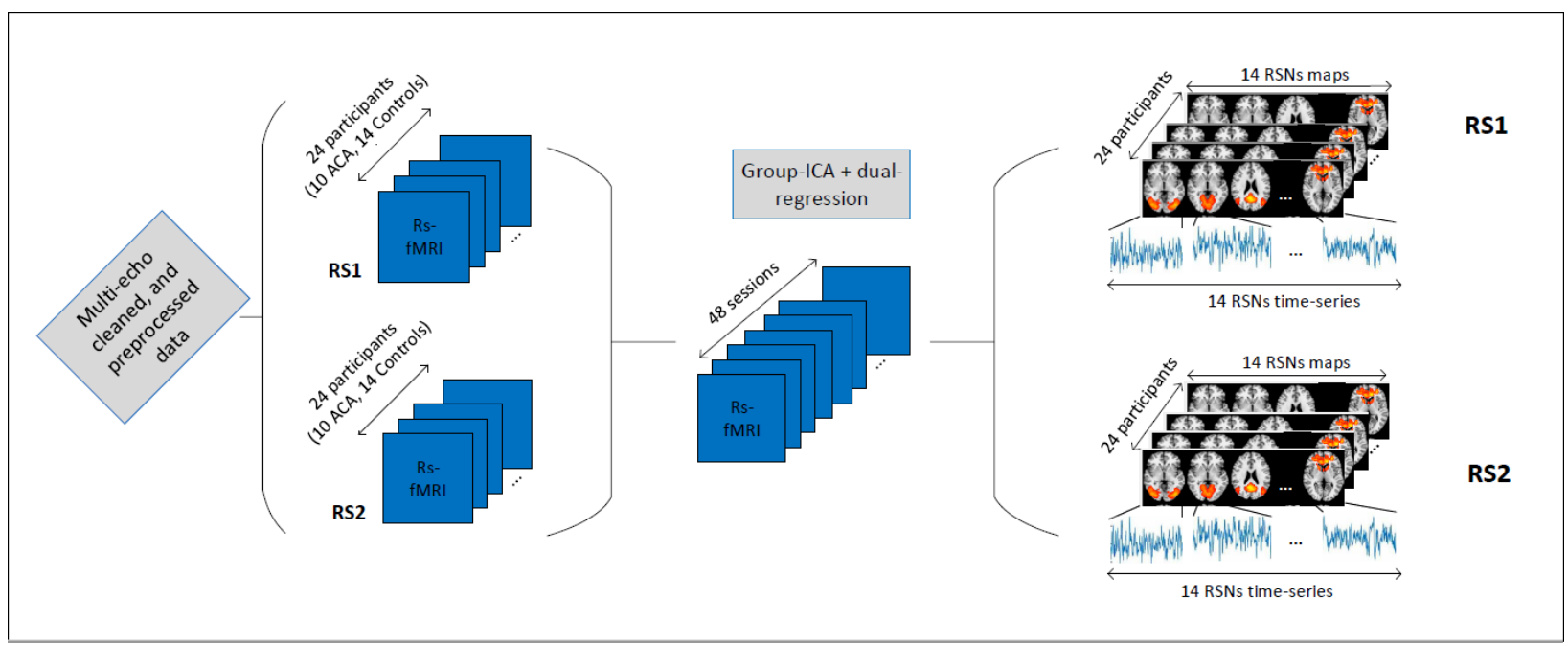

FIGURE 6.2: Brain networks (RSN) time series extraction.

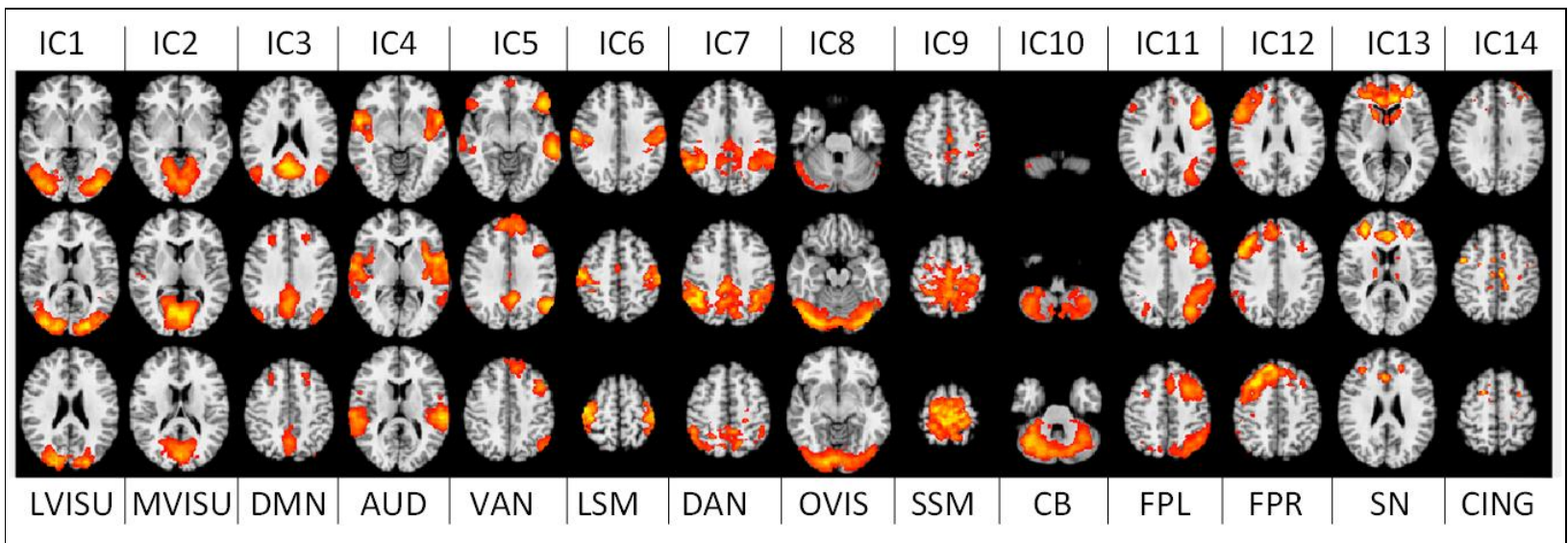

FIGURE 6.3: 14 large-scale brain functional resting-state networks (RSNs) used in the study. LVISU: lateral visual network; MVISU: medial visual network; DMN - default mode network; AUD - auditory network; VAN - ventral attention network; LSM - lateral sensory-motor network; DAN - dorsal attention network; OVIS - occipital visual network; SSM - superior sensory-motor network; CB - cerebellar network; FPL - left fronto-parietal network; FPR - right fronto-parietal network; SN - salience network; CING - the cingulate network.

\subsubsection{Static connectivity, causality, phase-coherence, and emulative power}

First, static functional connectivity ( $\mathrm{sFC}$ ) analysis was performed on all pairs of brain network signals, using Pearson correlation. This is applied for each participant's RSN time series, and each session (RS1 and RS2), leading to 2 (RS1 and RS2) times 24 (14 controls; 10 patients) correlation matrices. The total amount of sFC features per subject/session (unique entries of sFC matrix) was 91 ( = 14x13/2; the connectivity matrix being symmetrical, and its diagonal discarded).

Second, using the same ICs (or RSNs) time series we calculated the Granger causality (GC) matrices for each individual and resting-state session using the Matlab toolbox of (Barnett and Seth, 2014). A GC connectivity matrix is define as $\mathbf{G}=\left\{g_{i j}\right\} \in \mathbb{R}^{N \times N}$ ( $N=$ numbers of networks), where each matrix entry $g_{i j}$ represent the directed causal connectivity from network $\mathrm{j}$ towards network i. More specifically $g_{i j}=F_{Y \rightarrow X \mid Z}$, which is the GC between signal Y (time series of network $\mathrm{j}$ ) and $X$ (time series of network $\mathrm{i}$ ) conditional on $\mathrm{Z}$ (remaining time series of networks $k \neq i \neq j$ )-more details in (Bressler and Seth, 2011). The upper triangle of G, i.e. $\left\{g_{i j}\right\}, \forall j>i$ 
represent the inward causal pairwise connectivity $g_{i j}$, while the lower triangle, i.e. $\left\{g_{i j}\right\}, \forall i>j$ is all the outward causal connections of $\left\{g_{i j}\right\}$. Since GC matrices are asymmetrical (directed connectivity) by subtracting those triangular matrices, i.e lower-upper, we can extract the non-null net pairwise GC triangular matrix NetGC $=\left\{n e t g_{i j}\right\}=\left\{g_{i j}-g_{j i}\right\}, \forall i>j$. We also extracted the In- and Out-causal degree of each network by summing respectively the columns and rows of the G matrices, i.e. DegIn $=\left\{\sum_{j=1}^{N} g_{i j}\right\} \in \mathbb{R}^{N}$, and DegOut $=\left\{\sum_{i=1}^{N} g_{i j}\right\} \in \mathbb{R}^{N}$. We finally derived the net (out-in) causal degree of each network, i.e. NetDeg $=$ DegOut $-\operatorname{DegIn}{ }^{\mathrm{T}}$. Since we have $\mathrm{N}=14$ networks, the total amount of GC features was 315 ( = 3x91 (in-, out-, net-GC triangular matrices) + 3x14 (DegIn, DegOut, and NetDeg vectors)). These GC feature matrices and vectors were calculated per subject and session, and are illustrated in Figure 6.4.

Thirdly, we applied the wavelet-coherence approach with the following processing steps: (i) extraction of spectro-temporal maps (also called scalograms) of significant phase coherence, i.e. localised (in time and frequency/scales) significant phase-locked correlation between pairs of signals, as in Grinsted, Moore, and Jevrejeva, 2004. The phasing between the signals is expressed in radians and extracted from $\theta=\arg \left(W_{x y}\right)$, where $W_{x y}$ is a complex number defining the cross-wavelet transform between the two network signals being assessed. The phase information are summarised in 4 categories: in-phase when $\theta \in(-\pi / 4,+\pi / 4)$; anti-phase when $\theta \in(3 \pi / 4,-3 \pi / 4)$; and signal 1 leading (or lagging) signal 2 when $\theta \in(-3 \pi / 4,-\pi / 4)$ (or $\theta \in(\pi / 4,3 \pi / 4))$; for simplification we coloured the phase information in the wavelet-coherence maps (see Figure 6.4). For each of the 5 period scales $([4,8) \mathrm{s} ;[8,16) \mathrm{s} ;[16,32) \mathrm{s} ;[32,64) \mathrm{s}$; and $[64,128] \mathrm{s}$ ) we extracted the time of coherence (in \% of the scan duration) between the 91 pairs of networks. More details on the time of coherence metric in Bernas, Aldenkamp, and Zinger, 2018. the time of coherence extraction was performed for all of the 4 aforementioned phases. Therefore, in total we obtained $1820(=4 \times 5 \times 91)$ coherence-based features per subject's session. Finally, the Evolutionary Game theory on Network (EGN) was implemented as in Madeo et al., 2017. Instead of trying to predict brain signal (time series) dynamics and correlating them to the original BOLD time series, we here simply took the EGN-connectivity matrix $\mathbf{A}=\left\{a_{i j}\right\} \in \mathbb{R}^{N \times N}$ (N= numbers of networks), and derived the vectors of emulative powers. Briefly, each entry of $\mathbf{A}, a_{i j}$, described the pairwise and directed $(i \rightarrow j)$ weight of the network $i$ in its ability to emulate (positive value, $a_{i j}>0$ or not (negative value, $a_{i j}<0$ ) the activity of the network $\mathrm{j}$. A can be seen as similar to the GC connectivity matrices, albeit not in the sense of causality but in terms of replication mechanism. From these matrices of similar size $\left(N^{2}\right)$ as the SFC and GC matrices, we calculated our novel metrics, namely the In-, Out-, and Net-Emulative Powers (In-EPs, Out-EPs, and Net-EPs), per participants and session. The Out-EP of each network, i.e. its power to emulate (replicate) the other networks' activity is calculated by summing the columns of the EGN-connectivity matrix, i.e. $O u t E P_{i}=\sum_{j=1}^{N} a_{i j}, i \in\{1, . ., N\}$. The power of one network to be emulated (by the other networks) is represented by its in-EP, that is, the sum of the EGN-connectivity matrix rows, i.e. $\operatorname{InEP} P_{j}=\sum_{i=1}^{N} a_{i j}, j \in\{1, . ., N\}$. And the net replicative power of a network is defined by the net EP, i.e. NetEP $=$ OutEP $^{\mathrm{T}}-$ InEP. Since $N=14$, we ended up with three vectors of 14 EPs per person's rs-fMRI session, i.e. 42 features.

Figure 6.4 depicts the neurodynamics features extraction of the 4 approaches. These features were extracted for each RS session.

\subsubsection{Selection of the most relevant features and classification analysis}

After obtaining the subject-/session-specific sFC, GC, time of phase-coherence matrices, and NEP vectors, we performed non-parametric permutations tests (5000-permutations) for each entry of the mentioned matrices/vectors. The resulting matrices and vectors were then thresholded at $5 \%$ level $(\mathrm{p}<0.05)$, giving us the list of pairs of networks where the sFC, GC, time of coherence, and 


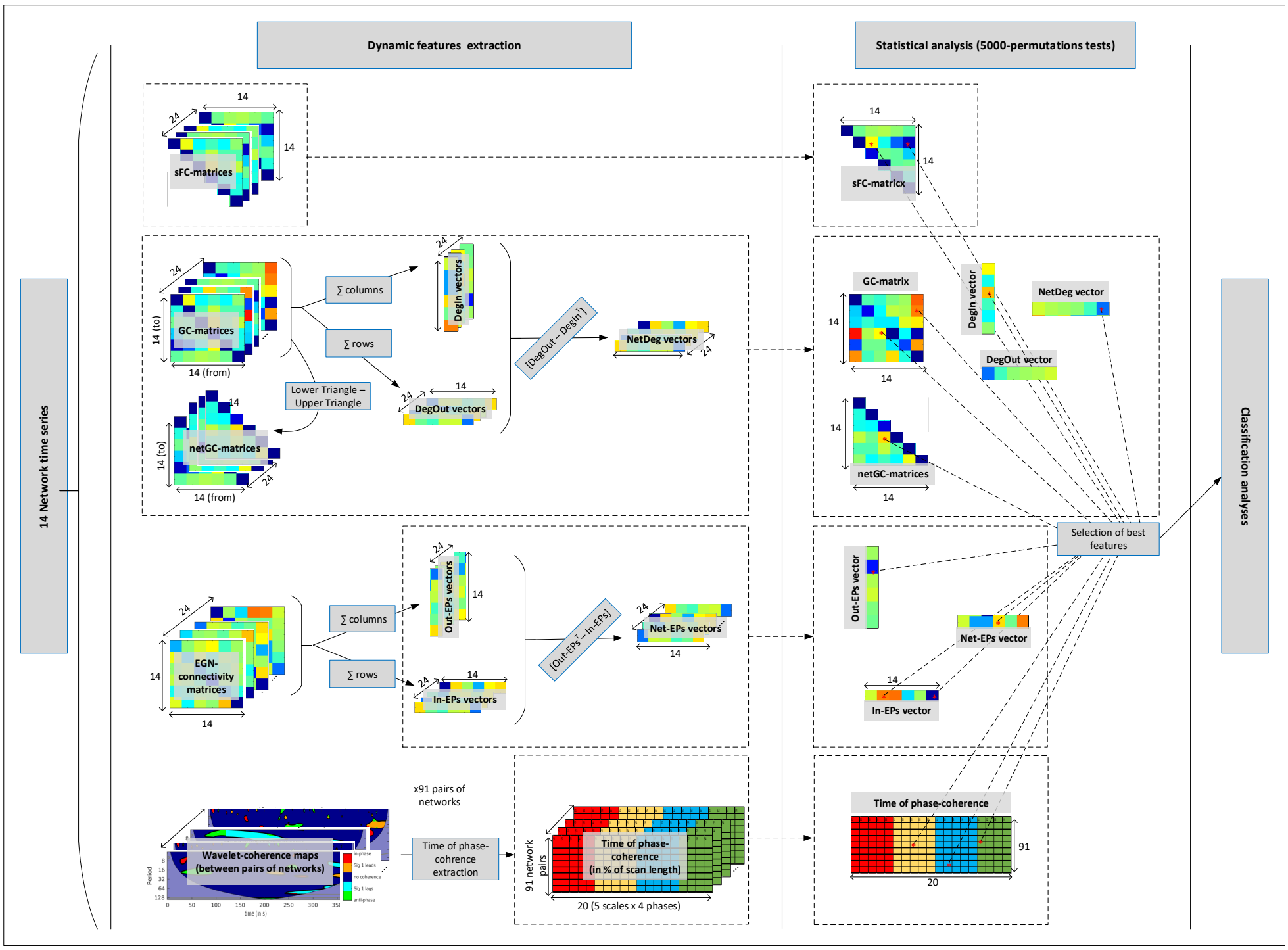

FIGURE 6.4: Dynamics feature extraction and statistical analysis.

NEPs were significantly different between the two groups (ACA and Controls). This statistical analysis was performed to reduce the total number of fMRI-based features to be used by the classifiers, which was initially 2268 (= 91 (sFC) 315 (GC) + 1820 (WC) + 42 (EGN)). This statistical analysis to obtain the best features is depicted in Figure 6.4.

In order to assess the strength of the abovementioned neurodynamics methods in describing an ACA in patients with epilepsy, classifiers were trained and validated. We used 4 types of machine learning, namely, the support vector machine (SVM), the linear discriminant analysis (LDA), the k-nearest neighborhood (KNN), and the decision tree (DT) algorithms. To validate the classifiers, 5 methods were used: validation on RS2 features (with classifiers trained on RS1's); validation on RS1 features (with classifiers trained on RS2's); leave-on-out cross-validation (LOOCV) in RS1 (24-folds cross-validation); LOOCV in RS2 (24-folds); LOOCV in RS1 and RS2 concatenated (48folds). For each of the classifier types of training and validation, different set-ups of features were used. First, we tested the classifiers using the best features (after statistical analyses) from one type of dynamics at a time, i.e. only best features from $\mathrm{sFC}$ analysis, then only significant features from GC analysis, and so on. Next, we refined these set-ups to the use of features that fell into two categories: (i) features/predictors that were used in the DT algorithm, i.e., selected by the Classification And Regression Trees (CART; default split predictor selection technique for classification 
trees in Matlab); (ii) features that involved the DMN, DAN or SN (networks often mentioned in ageing and cognitive decline literature; see Introduction). Then, we reiterated refinements of the classifier setups by removing or adding features that still comply with the aforementioned criteria ( $\mathrm{i}$ and ii), if they were improving classification accuracy in the previous refinement iterations. Such a search for the best combinations of features led us to a dozen of classification analyses per dynamics metrics. Finally, we tested classifiers with combined dynamics features, e.g. using the best GC-based features combined with the best WCoh-based features. We refined the classification models the same way it was performed in the classification analyses per dynamics metric. We also tried the combination of all best features, i.e. combining all features selected by the statistical analyses of all dynamics metrics. In the combined dynamics metrics setups, a dozen (through refinements) classifiers were also tested. For each of the classifier setup tested $(60=5 \times 12)$, we reported the accuracy, sensitivity and specificity. For clarity, in the Results section, only the best classifiers performances per dynamics, and per machine learning algorithms are reported, i.e. the highest average accuracy of the classifiers over the 5 validation methods.

\subsection{Results}

\subsubsection{Selection of the best dynamics features}

After extracting all static and dynamic features (sFC, GC, WCoh-, EGN), 5000-permutation tests between the two groups' features were applied and thresholded at (uncorrected) $p<0.05$, giving us the most significant differences between the groups, i.e. the 'best' features. The results of the significant permutation tests for sFC-, GC- and EGN-based features are shown in Appendix C Figures C.1- C.4.

Regarding the WCoh-based features, namely the time-of-coherence measurements, the permutation tests gave us an average of more than 50 significant features per phase (in-phase, anti-phase, leading, and lagging). In order to avoid an overfitting problem, we reduced the number of best features even further, as follows: (i) discarding features that also showed significant differences in controls when comparing RS1 and RS2; (ii) keeping features that showed robustness, i.e. significant in several scales, or in multiple phases; or (iii) time-of-coherence with higher evidence of between-group differences $(\mathrm{p}<0.01)$. This led us to having an average of 10 best features per phase. An example of a pairwise (in-phase) time-of-coherence that complied with the aforementioned criteria is depicted in Figure 6.5.

All of the best features for each neurodynamics metric and their labels are summarised in Table 6.2.
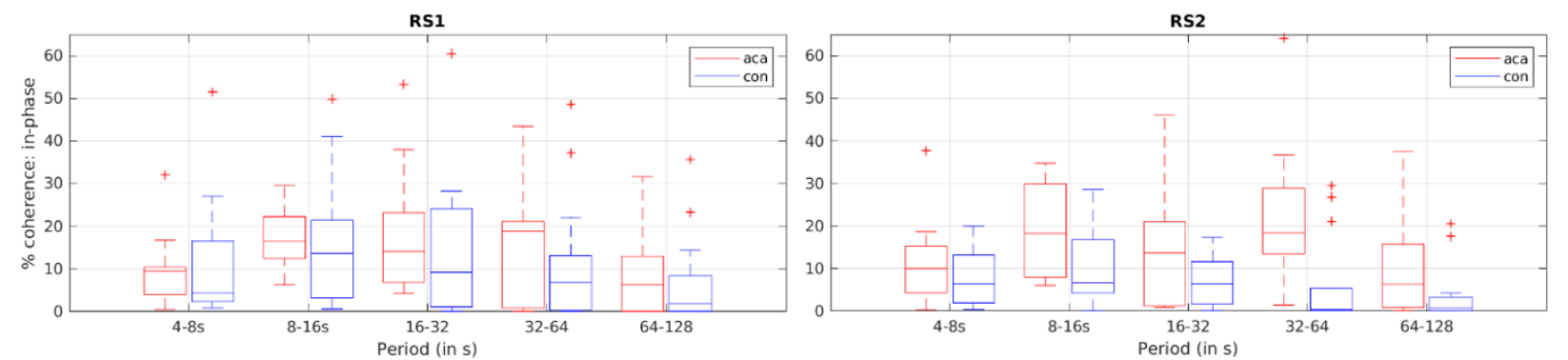

FIGURE 6.5: Example of \% time of in-phase coherence per group (ACA, Controls) between MVISU and DMN. Red boxplot - ACA; blue boxplot - controls; left - RS1; right - RS2. 
TABLE 6.2: List of all 86 best features used in the classification analyses

\begin{tabular}{|c|c|c|c|c|c|}
\hline Wavelet coherence & & Granger causality & & Static FC & EGN \\
\hline $\begin{array}{l}\text { in-phase time-of-coherence } \\
\text { ip LVISU-OVIS s1 } \\
\text { ip LSM-CING s1 } \\
\text { ip OVIS-SN s2 } \\
\text { ip CB-SN s2 } \\
\text { ip MVISU-DMN s2 } \\
\text { ip OVIS-SN s3 } \\
\text { ip CB-SN s3 } \\
\text { ip DMN-DAN s4 } \\
\text { ip MVISU-DMN s4 } \\
\text { anti-phase time-of-coherence } \\
\text { ap AUDI-FPR s1 } \\
\text { ap VAN-FPL s1 } \\
\text { ap AUDI-SSM s1 } \\
\text { ap DMN-VAN s2 } \\
\text { ap OVIS-SN s2 } \\
\text { ap LVISU-VAN s3 } \\
\text { ap OVIS-SN s3 } \\
\text { ap VAN-SN s4 } \\
\text { ap DMN-AUDI s5 }\end{array}$ & $\begin{array}{l}\text { leading time-of-coherence } \\
\text { lead AUDI-FPR s1 } \\
\text { lead MVISU-DMN s1 } \\
\text { lead MVISU-OVIS s2 } \\
\text { lead DMN-OVIS s2 } \\
\text { lead LSM-OVIS s2 } \\
\text { lead LSM-OVIS s3 } \\
\text { lead OVIS-CB s3 } \\
\text { lead LVISU-VAN s4 } \\
\text { lead DMN-VAN s4 } \\
\text { lead FPL-SN s4 } \\
\text { lagging time-of-coherence } \\
\text { lag LVISU-DAN s1 } \\
\text { lag OVIS-SSM s1 } \\
\text { lag OVIS-SN s2 } \\
\text { lag OVIS-FPL s2 } \\
\text { lag CB-FPR s2 } \\
\text { lag CB-FPL s2 } \\
\text { lag LSM-OVIS s3 } \\
\text { lag VAN-CB s3 }\end{array}$ & $\begin{array}{l}\text { net1 } \rightarrow \text { net2 GC } \\
\text { MVISU } \rightarrow \text { LVISU } \\
\text { MVISU } \rightarrow \text { FPL } \\
\text { MVISU } \rightarrow \text { CING } \\
\text { DMN } \rightarrow \text { AUDI } \\
\text { DMN } \rightarrow \text { SN } \\
\text { VAN } \rightarrow \text { CING } \\
\text { DAN } \rightarrow \text { FPR } \\
\text { AUDI } \rightarrow \text { SN } \\
\text { LSM } \rightarrow \text { CING } \\
\text { FPL } \rightarrow \text { FPR } \\
\text { FPL } \rightarrow \text { CING } \\
\text { net DegIn, DegOut } \\
\text { and netDeg } \\
\text { GC DegOut LSM } \\
\text { GC NetDeg DAN } \\
\text { GC NetDeg AUDI } \\
\text { GC NetDeg LSM }\end{array}$ & $\begin{array}{l}\text { net1 } \leftarrow \text { net2 GC } \\
\text { MVISU } \leftarrow \text { LSM } \\
\text { MVISU } \leftarrow \text { FPR } \\
\text { LVISU } \leftarrow \text { AUDI } \\
\text { LVISU } \leftarrow \text { SSM } \\
\text { LVISU } \leftarrow \text { SN } \\
\text { VAN } \leftarrow \text { DAN } \\
\text { VAN } \leftarrow \text { AUDI } \\
\text { DAN } \leftarrow \text { CING } \\
\text { AUDI } \leftarrow \text { SSM } \\
\text { net pairwise GC } \\
\text { netgc MVISU } \rightarrow \text { LVISU } \\
\text { netgc MVISU } \rightarrow \text { CING } \\
\text { netgc LVISU } \rightarrow \text { LSM } \\
\text { netgc LVISU } \rightarrow \text { SN } \\
\text { netgc DMN } \rightarrow \text { VAN } \\
\text { netgc DMN } \rightarrow \text { AUDI } \\
\text { netgc DAN } \rightarrow \text { CING } \\
\text { netgc FPL } \rightarrow \text { FPR }\end{array}$ & $\begin{array}{l}\text { net1 } \leftrightarrow \text { net2 } \\
\text { sFC-DMN-VAN } \\
\text { sFC-MVISU-DMN } \\
\text { sFC-DMN-LSM } \\
\text { sFC-DMN-CB } \\
\text { sFC-AUDI-CING } \\
\text { sFC-LSM-OVISU } \\
\text { sFC-DAN-SN } \\
\text { sFC-OVISU-SN } \\
\text { sFC-CB-FPL } \\
\text { sFC-CB-CING }\end{array}$ & $\begin{array}{l}\text { EPs } \\
\text { InEP DMN } \\
\text { InEP DAN } \\
\text { InEP SSM } \\
\text { InEP CING } \\
\text { OutEP DMN } \\
\text { OutEP OVIS } \\
\text { NetEP DMN } \\
\text { NetEP CING }\end{array}$ \\
\hline
\end{tabular}

\subsubsection{Classification performances}

After static (sFC) and dynamic analyses (GC, WC, EGN) followed by the statistical permutation testing we obtained the best descriptors (features) for each dynamics metric. All and subsets of these best features were used for training and validating classifiers. In Table 6.3, the best performances of each individual dynamics metrics are shown. For wavelet-coherence, the in-phase time of coherence were the best descriptors of ACA, reaching an average $77.1 \%$ accuracy with the decision-tree algorithm, using DMN, SN and visual networks. Using 4 DMN-based and 2 SN-based static connectivity features, the average classification accuracy was $77.9 \%$, with LDA algorithm. With the emulative powers (EGN-based dynamics metric, setup \#1) of the DMN and the DAN we could reach $82.9 \%$ accuracy, also using LDA algorithm. Note that the out-EP and net-EP of the DAN (used in setup \#1; see Table C.1) were not part of selected best features (see Table 6.2), but interestingly improved the classification accuracy when added. GC-based features, however, led only to $67.9 \%$ accuracy with DT algorithm. Overall the metrics giving the best results for classification can be ranked as EPs $>$ sFC $>$ in-phase coherence $>$ GC.

After assessing the best features of each dynamics metric, we also trained and tested classifiers with combinations of the features. This gave us the best performances for each classifier type. LDA reached $85 \%$ average accuracy using all 86 best features (see Table 6.2). KNN led to an average of $80.8 \%$ accuracy with a mix of 82 features that is the 86 best features minus 4 Eps: out-Eps of DMN and OVIS; and net-Eps of DMN and CING. DT algorithm reached $80.0 \%$ with only 6 features ( 3 Coherence, 2 EGN, and $1 \mathrm{sFC}$ ). And SVM had the best results with 15 features ( 2 in-phase coherences, 4 in-EPs, $3 \mathrm{GC}$, and $6 \mathrm{sFC}$ ) reaching average accuracy of $90.0 \%$. In the latter SVM setup, it is worth noting that the independent training and validation, i.e. RS1-trained RS2-validated, and vice-versa, could classify ACA at $91.7 \%$ accuracy; the 48 -fold LOOCV had $95.8 \%$ accuracy. Finally, the overall best average accuracy $(81.0 \%)$, over all the machine-learning algorithms, was obtained using even fewer features (10: 2 in-phase coherence, 2 in-EPs, and $6 \mathrm{sFC}$ ). These results are depicted in Table 6.4. Looking at the classifier algorithms, in general, SVM was performing the best. With few features $(<10)$, DT was performing quite well, but still not as good as SVM and LDA. KNN was overall the least accurate classification algorithm. It is worth noting that in some set-ups DT algorithm reached 100\% accuracy, such as for the LOOCV of RS2 with the mix of 6 features (see Table 6.4); but this was not consistent, since in the same set-up (of 6 features), the RS1-trained-and-RS2-validated classifier reached only 66.7\% accuracy. Overall, EGN-based net 
TABLE 6.3: Summary of the best classifier performances for each dynamic measures: EGN, WCoh, sFC, and GC

\begin{tabular}{|c|c|c|c|c|c|c|c|c|c|c|c|c|c|}
\hline Features & Valid. meth. & & SVM & & & LDA & & & KNN & & & DT & \\
\hline \multirow{3}{*}{ EGN } & & acc & sen & spe & acc & sen & spe & acc & sen & spe & acc & sen & spe \\
\hline & tRS1-vRS2 & 0.917 & 900 & 0.929 & 0.917 & 0.900 & 0.929 & 0.708 & 0.500 & 0.857 & 0.750 & 0.700 & 0.786 \\
\hline & tRS2- & 0.708 & 0.700 & 0.714 & 0.833 & 0.800 & 0.857 & 0.708 & 0.600 & 0.786 & 0.583 & 0.600 & 0.571 \\
\hline \multirow[t]{4}{*}{ [setup \#1 ${ }^{*}$ ] } & Loocv-RS1 & 0.750 & 0.700 & 0.786 & 0.750 & 0.700 & 0.786 & 0.375 & 0.300 & 0.429 & 0.583 & 0.600 & 0.571 \\
\hline & Loocv & 0.750 & 0.700 & 0.786 & 0.833 & 0.800 & 0.857 & 0.625 & .500 & 0.714 & 0.542 & 300 & 0.714 \\
\hline & Loocv-conc & 0.771 & 0.750 & 0.786 & 0.813 & 0.750 & 0.857 & 0.708 & 0.600 & 0.786 & 0.771 & 0.700 & 0.821 \\
\hline & Avera & 0.779 & 0.750 & 0.800 & 0.829 & 0.790 & 0.857 & 0.625 & 0.500 & 0.714 & 0.646 & 0.580 & 0.693 \\
\hline \multirow{3}{*}{ WCoh } & & acc & st & $\mathrm{sp}$ & acc & $\mathrm{s}$ & & acc & & st & acc & & spe \\
\hline & tRS1- & 0.417 & 1.000 & 0.000 & 0.917 & 0.800 & 1.000 & 0.583 & 0.700 & 0.500 & 0.750 & & 0.571 \\
\hline & tRS2 & 0.417 & 1.000 & 0.000 & 0.708 & 0.700 & 0.714 & 0.750 & 0.900 & 0.643 & 0.667 & .500 & 0.786 \\
\hline \multirow[t]{4}{*}{ [setup \#2 ${ }^{*}$ ] } & Loocv-RS1 & 0.417 & 1.000 & 0.000 & 0.417 & 1.000 & 0.000 & 0.625 & 0.600 & 0.643 & 0.708 & 0.800 & 0.643 \\
\hline & Looct & 0.417 & 1.000 & 0.000 & 0.792 & 0.700 & 0.857 & 0.875 & 0.900 & 0.857 & 1.000 & 000 & 1.000 \\
\hline & Loocv-conca & 0.417 & 1.000 & 0.000 & 0.729 & 0.650 & 0.786 & 0.729 & 0.750 & 0.714 & 0.729 & .750 & 0.714 \\
\hline & Ave & 0.417 & 1.000 & 0.000 & 0.713 & 0.770 & 0.671 & 0.713 & 0.770 & 0.671 & 0.771 & 0.810 & 0.743 \\
\hline \multirow{3}{*}{$\mathrm{sFC}$} & & acc & ser & $\mathrm{sp}$ & $\mathrm{acc}$ & se & $\mathrm{s}_{1}$ & $\mathrm{ac}$ & se & $\mathrm{sp}$ & acc & & spe \\
\hline & tRS1 & 0.875 & 0.700 & 1.000 & 0.875 & 0.700 & 1.000 & 0.875 & 0.800 & 0.929 & 0.458 & & 0.500 \\
\hline & tRS2 & 0.750 & 0.900 & 0.643 & 0.792 & 0.900 & 0.714 & 0.750 & 1.000 & 0.571 & 0.542 & 700 & 0.429 \\
\hline \multirow[t]{4}{*}{ [setup \#3*] } & Loo & 0.667 & 0.600 & 0.714 & 0.667 & 0.600 & 0.714 & 0.583 & 0.400 & 0.714 & 0.625 & 500 & 0.714 \\
\hline & Looc & 0.833 & 0.700 & 0.929 & 0.750 & 0.600 & 0.857 & 0.750 & 0.500 & 0.929 & 0.833 & 0.700 & 0.929 \\
\hline & conca & 0.771 & 0.700 & 0.821 & 0.750 & 0.700 & 0.786 & 0.750 & 0.550 & 0.893 & 0.583 & 0.500 & 0.643 \\
\hline & Average & 0.779 & 0.720 & 0.821 & 0.767 & 0.700 & 0.814 & 0.742 & 0.650 & 0.807 & 0.608 & 0.560 & 0.643 \\
\hline \multirow{3}{*}{ GC } & & & sel & $\mathrm{sp}$ & $\mathrm{acc}$ & se & spe & $\mathrm{acc}$ & ser & $\mathrm{sp}$ & a & & spe \\
\hline & tRS1- & 0.417 & 1.000 & 0.000 & 0.542 & 0.300 & 0.714 & 0.500 & 0.200 & 0.714 & 0.667 & 0.400 & 0.857 \\
\hline & tRS2- & 0.417 & 1.000 & 0.000 & 0.708 & 0.700 & 0.714 & 0.500 & 0.400 & 0.571 & 0.667 & 0.600 & 0.714 \\
\hline \multirow[t]{4}{*}{ [setup \#4 $4^{*}$ ] } & Loocv-RS1 & 0.417 & 1.000 & 0.000 & 0.417 & 1.000 & 0.000 & 0.625 & 0.600 & 0.643 & 0.708 & 0.600 & 0.786 \\
\hline & & 0.417 & 1.000 & 0.000 & 0.458 & 0.600 & 0.357 & 0.542 & 0.6 & 0.500 & 0.750 & 00 & 0.714 \\
\hline & & 0.4 & & & 0.729 & & & 0.563 & & & 0.604 & 50 & 0.643 \\
\hline & Average & 0.417 & 1.000 & 0.000 & 0.571 & 0.660 & 0.507 & 0.546 & 0.460 & 0.607 & 0.679 & 0.590 & 0.743 \\
\hline
\end{tabular}

Grey highlight = accuracy values; yellow highlight = best averages accuracy in each dynamics method; blue highlight = accuracy $>80 \%$. SVM - support vector machine; LDA - linear discriminant analyses; KNN - K-nearest neighbours; DT - decision tree. acc accuracy; sen - sensitivity; spe - specificity; tRS1(2)-vRS2(1) - trained on RS1(or 2) and validated on RS2(or 1); Loocv - leave one-out cross-validation; conca - concatenation of RS1 and RS2 features. ${ }^{*}$ Setups with the features used for classification can be found in Appendix Table C.1.

emulative powers are the best descriptors, especially when using DMN and DAN features. And when those are combined with specific time of in-phase coherence and static connectivity (correlation) values, SVM algorithm outperforms the other algorithm with an average of $90.0 \%$ accuracy; with peaks at $91.7 \%$ for the RS-independent training/validation classifications, and $95.8 \%$ in the 48-folds LOOCV. 
TABLE 6.4: Summary of the best performances of each classifier algorithm: SVM, LDA, KNN, and DT

\begin{tabular}{|c|c|c|c|c|c|c|c|c|c|c|c|c|c|}
\hline \multirow[t]{2}{*}{ Features } & Valid. meth. & \multicolumn{3}{|c|}{ SVM } & \multicolumn{3}{|c|}{ LDA } & \multicolumn{3}{|c|}{ KNN } & \multicolumn{3}{|c|}{ DT } \\
\hline & & acc & sen & spe & acc & sen & spe & acc & sen & spe & acc & sen & spe \\
\hline \multirow{4}{*}{$\begin{aligned} & 1 \mathrm{EGN} \\
+ & 3 \mathrm{WCoh} \\
+ & 2 \mathrm{sFC}\end{aligned}$} & tRS1-vRS2 & 0.792 & 0.500 & 1.000 & 0.750 & 0.600 & 0.857 & 0.833 & 0.800 & 0.857 & 0.833 & 1.000 & 0.714 \\
\hline & tRS2-vRS1 & 0.792 & 0.600 & 0.929 & 0.792 & 0.900 & 0.714 & 0.792 & 0.800 & 0.786 & 0.667 & 0.500 & 0.786 \\
\hline & Loocv-RS1 & 0.833 & 0.700 & 0.929 & 0.833 & 0.700 & 0.929 & 0.833 & 0.900 & 0.786 & 0.750 & 0.600 & 0.857 \\
\hline & Loocv-RS2 & 0.708 & 0.300 & 1.000 & 0.708 & 0.600 & 0.786 & 0.667 & 0.600 & 0.714 & 1.000 & 1.000 & 1.000 \\
\hline \multirow[t]{2}{*}{ [setup \#5 $]$} & Loocv-conca & 0.792 & 0.600 & 0.929 & 0.792 & 0.700 & 0.857 & 0.750 & 0.650 & 0.821 & 0.750 & 0.700 & 0.786 \\
\hline & Average & 0.783 & 0.540 & 0.957 & 0.775 & 0.700 & 0.829 & 0.775 & 0.750 & 0.793 & 0.800 & 0.760 & 0.829 \\
\hline \multirow{7}{*}{$\begin{aligned} & 4 \mathrm{EGN} \\
+ & 36 \mathrm{WCoh} \\
+ & 32 \mathrm{GC} \\
+ & 10 \mathrm{sFC} \\
& {[\text { setup \#6 }}\end{aligned}$} & & acc & sen & spe & acc & sen & spe & acc & sen & spe & acc & sen & spe \\
\hline & tRS1-vRS2 & 0.917 & 0.800 & 1.000 & 0.792 & 0.600 & 0.929 & 0.750 & 0.800 & 0.714 & 0.750 & 0.400 & 1.000 \\
\hline & tRS2-vRS1 & 0.917 & 0.800 & 1.000 & 0.833 & 0.800 & 0.857 & 0.833 & 0.700 & 0.929 & 0.667 & 0.500 & 0.786 \\
\hline & Loocv-RS1 & 0.750 & 0.700 & 0.786 & 0.750 & 0.700 & 0.786 & 0.750 & 0.700 & 0.786 & 0.458 & 0.300 & 0.571 \\
\hline & Loocv-RS2 & 0.792 & 0.700 & 0.857 & 0.958 & 0.900 & 1.000 & 0.917 & 0.800 & 1.000 & 0.958 & 1.000 & 0.929 \\
\hline & Loocv-conca & 0.938 & 0.850 & 1.000 & 0.833 & 0.750 & 0.893 & 0.792 & 0.700 & 0.857 & 0.542 & 0.450 & 0.607 \\
\hline & Average & 0.863 & 0.770 & 0.929 & 0.833 & 0.750 & 0.893 & 0.808 & 0.740 & 0.857 & 0.675 & 0.530 & 0.779 \\
\hline \multirow{7}{*}{$\begin{aligned} & 8 \mathrm{EGN} \\
+ & 36 \mathrm{WCoh} \\
+ & 32 \mathrm{GC} \\
+ & 10 \mathrm{sFC} \\
& {\left[\text { setup } \# 7^{*} \text { ] }\right.}\end{aligned}$} & & acc & sen & spe & acc & sen & spe & acc & sen & spe & acc & sen & spe \\
\hline & tRS2-vRS1 & 0.750 & 0.600 & 0.857 & 0.792 & 0.600 & 0.929 & 0.750 & 0.700 & 0.786 & 0.750 & 0.400 & 1.000 \\
\hline & Loocv-RS1 & 0.667 & 0.800 & 0.571 & 0.875 & 0.800 & 0.929 & 0.750 & 0.700 & 0.786 & 0.667 & 0.500 & 0.786 \\
\hline & Loocv-RS1 & 0.833 & 0.700 & 0.929 & 0.833 & 0.700 & 0.929 & 0.583 & 0.400 & 0.714 & 0.458 & 0.300 & 0.571 \\
\hline & Loocv-RS2 & 0.875 & 0.800 & 0.929 & 0.917 & 0.800 & 1.000 & 0.583 & 0.400 & 0.714 & 0.917 & 0.900 & 0.929 \\
\hline & Loocv-conca & 0.917 & 0.800 & 1.000 & 0.833 & 0.750 & 0.893 & 0.688 & 0.500 & 0.821 & 0.542 & 0.450 & 0.607 \\
\hline & Average & 0.808 & 0.740 & 0.857 & 0.850 & 0.730 & 0.936 & 0.671 & 0.540 & 0.764 & 0.667 & 0.510 & 0.779 \\
\hline \multirow{7}{*}{$\begin{aligned} & 4 \mathrm{EGN} \\
+ & 2 \mathrm{WCoh} \\
+ & 3 \mathrm{GC} \\
+ & 6 \mathrm{sFC} \\
& {\left[\text { setup } \# 8^{*}\right] }\end{aligned}$} & \multirow{7}{*}{$\begin{array}{l}\text { tRS1-vRS2 } \\
\text { tRS2-vRS1 } \\
\text { Loocv-RS1 } \\
\text { Loocv-RS2 } \\
\text { Loocv-conca } \\
\text { Average }\end{array}$} & acc & sen & spe & acc & sen & spe & acc & sen & spe & acc & sen & spe \\
\hline & & 0.917 & 0.800 & 1.000 & 0.917 & 0.800 & 1.000 & 0.708 & 0.500 & 0.857 & 0.708 & 0.600 & 0.786 \\
\hline & & 0.917 & 0.800 & 1.000 & 0.583 & 0.500 & 0.643 & 0.708 & 0.600 & 0.786 & 0.667 & 0.600 & 0.714 \\
\hline & & 0.833 & 0.700 & 0.929 & 0.833 & 0.700 & 0.929 & 0.500 & 0.300 & 0.643 & 0.708 & 0.600 & 0.786 \\
\hline & & 0.875 & 0.800 & 0.929 & 0.708 & 0.500 & 0.857 & 0.875 & 0.800 & 0.929 & 0.792 & 0.800 & 0.786 \\
\hline & & 0.958 & 0.900 & 1.000 & 0.875 & 0.850 & 0.893 & 0.729 & 0.550 & 0.857 & 0.792 & 0.800 & 0.786 \\
\hline & & 0.900 & 0.800 & 0.971 & 0.783 & 0.670 & 0.864 & 0.704 & 0.550 & 0.814 & 0.733 & 0.680 & 0.771 \\
\hline \multirow{5}{*}{$\begin{aligned} & 2 \mathrm{EGN} \\
+ & 2 \mathrm{WCoh} \\
+ & 6 \mathrm{sFC}\end{aligned}$} & & acc & sen & spe & acc & sen & spe & acc & sen & spe & acc & sen & spe \\
\hline & tRS1-vRS2 & 0.875 & 0.700 & 1.000 & 0.917 & 0.800 & 1.000 & 0.875 & 0.700 & 1.000 & 0.833 & 1.000 & 0.714 \\
\hline & tRS2-vRS1 & 0.917 & 0.800 & 1.000 & 0.792 & 0.800 & 0.786 & 0.792 & 0.600 & 0.929 & 0.792 & 0.600 & 0.929 \\
\hline & Loocv-RS1 & 0.750 & 0.500 & .929 & 0.750 & 0.500 & 0.929 & 0.625 & 0.700 & 0.571 & 0.792 & 0.700 & 0.857 \\
\hline & Loocv-RS2 & 0.875 & 0.800 & 0.929 & 0.708 & 0.500 & 0.857 & 0.792 & 0.700 & 0.857 & 0.667 & 0.500 & 0.786 \\
\hline \multirow[t]{3}{*}{ [setup \#9*] } & Loocv-conca & 0.917 & 0.800 & 1.000 & 0.875 & 0.850 & 0.893 & 0.854 & 0.750 & 0.929 & 0.813 & 0.700 & 0.893 \\
\hline & Average & 0.867 & 0.720 & 0.971 & 0.808 & 0.690 & 0.893 & 0.788 & 0.690 & 0.857 & 0.779 & 0.700 & 0.836 \\
\hline & overall average & 0.810 & & & & & & & & & & & \\
\hline
\end{tabular}

Grey highlight = accuracy values; yellow highlight = best averages accuracy in each set-up; blue highlight = accuracy $>80 \%$. SVM support vector machine; LDA - linear discriminant analyses; KNN - K-nearest neighbours; DT - decision tree; acc - accuracy; sen sensitivity; spe - specificity; tRS1(2)-vRS2(1) - trained on RS1(or 2) and validated on RS2(or 1); Loocv - leave one-out cross-validation; conca - concatenation of RS1 and RS2 features. ${ }^{*}$ Setups with the features used for classification can be found in Appendix Table C.1.

\subsection{Discussion}

\subsubsection{Clinical relevance}

We have shown that the emulative powers (Eps) of the default mode network and the dorsal attention networks are biomarkers of ACA. Combining these features with DMN and SN-related in-phase coherence, and static functional connectivity would allow diagnosing the disorder at an overall 81.0\% accuracy (average of all machine-learning algorithms tested). Adding CING- and SSM-related EPs and DAN-, AUDI-, and LSM-related GCs, could even improve SVM accuracy up to $95.8 \%$. This shows the following: (i) dynamics metrics performs better than correlation and causal metrics at classifying an ACA in patients with epilepsy; and (ii) combining them with static functional connectivity improves the classifications. This is in line with a recent meta-analysis on the merit of using dynamic functional connectivity to describe psychological outcomes, especially when performance is assessed with objective measures such as IQ tests-as compared to more subjective (self-reported) psychological assessment (Liégeois et al., 2019). 
In the clinical context, the default mode, dorsal attention networks, and the salience network being involved and informative to distinguish neuropsychiatric patients from controls is already shown in previous research. In ageing the DMN and DAN are often mentioned and know to be challenged (Damoiseaux et al., 2008; Ferreira et al., 2016; Onoda, Ishihara, and Yamaguchi, 2012a). Also the dynamics in terms of causality is shown to correlate with psychological performances (Tsvetanov et al., 2016). Static and dynamic functional connectivity of the DMN and DAN might be explained by the compensatory mechanism observed in aging, as described in the CRUNCH model (Reuter-Lorenz and Cappell, 2008; Sala-Llonch, Bartrés-Faz, and Junqué, 2015). This compensatory mechanism is defined as the cognitive reserve in the study of (Bastin et al., 2012) wherein DMN and DAN are also involved. Other studies have linked cognitive decline with changes in static and dynamic functional connectivity between the DMN and DAN (Damoiseaux et al., 2008; Onoda, Ishihara, and Yamaguchi, 2012b; Tomasi and Volkow, 2012). The salience network (SN), which was part of our sFC best features, and its overall decreased in functional connectivity, is also a marker of brain ageing (Onoda, Ishihara, and Yamaguchi, 2012a; Sala-Llonch, Bartrés-Faz, and Junqué, 2015). SN has also a pivotal role in predicting age and cognitive performances (ranging from episodic memory to executive functions), in healthy elderly with cognitive decline (La Corte et al., 2016). Regarding mild cognitive impairment, which is a disorder wherein symptoms resemble the deficit in ACA patients, the sFC showed also disturbed network activity in the aforementioned brain regions (Farràs-Permanyer, Guàrdia-Olmos, and Peró-Cebollero, 2015; Zhenyu Liu et al., 2012). Finally in a review paper, from 81 fMRI studies where elderly had a significant cognitive decline, $50 \%$ of the studies showed changes in brain activation occurring in the DAN, and 15.3\% in the DMN (Li et al., 2015). Sensorimotor networks are also implicated in the process of cognitive ageing (Siman-Tov et al., 2017). That explains the involvement of the LSM-DMN sFC, the Net GC degree of the LSM, and the In-EP of the SSM, in our best classifier set-up. Finally, the time of in-phase coherence between the pair MVISU-DMN often helped the performances of our classifiers. This might be explained by the anterior-posterior shift observed in ageing in the PASA model of the ageing brain (Davis et al., 2008). This shift involved an overrecruitment of the posterior brain functional network (here DMN-MVISU connectivity) in order to compensate for the 'normal' decline in ageing of the visual abilities. And this correlates negatively with the cognitive performances, similar to the increase of (in-phase) coherence in ACA patients with deterioration in IQ. ACA patient suffers more specifically from a deterioration in fluid intelligence, which is described as the ability to think logically and solve problems with a limited amount of task-related information (Ezaki et al., 2020). The aforementioned DMN, CING (for cognitive control), and sensorimotor (LSM/SSM) networks have been shown to be linked to this type of intelligence/cognitive processing (Cole et al., 2012). The fluid intelligence might come from the ability to switch from perceptive network to processing network, and the ability to switch and hold attention. The DMN, SN and DAN are the 3 networks involved in self-referential processes, dynamic switching between rest and task, and the voluntary allocation of attention (Beason-Held, Kraut, and Resnick, 2009; Menon and Uddin, 2010; Vossel, Geng, and Fink, 2014). Having less copying mechanism here (as shown by the EGN dynamics) in those networks, might reflect a more rigid brain processing, i.e. less fluidic integration, which might explain the poor results in Perceptual Reasoning Index (PRI; fluid intelligence test). Overall, the DMN, DAN, SN and their dynamic and static functional connectivity to multiple sensory-related networks (visual, sensorimotor) could, no matter the resting-state, help classify correctly patients from healthy subjects. And the involvement of these networks are in line with current research on cognitive decline in ageing, and brain functional connectivity (Andrews-Hanna et al., 2007; Chand et al., 2017; La Corte et al., 2016; Li et al., 2015; Luo et al., 2020; Sala-Llonch, Bartrés-Faz, and Junqué, 2015; Tomasi and Volkow, 2012; Tsvetanov et al., 2016). 


\subsubsection{Technical insights}

Evolutionary game theory on networks and its derived emulative powers were the best at describing (highest classifying performances) ACA as compared to the other metrics used in this study. More specifically the In-emulative power of the DMN and DAN were strong biomarkers of ACA. In general (in average for RS1 and RS2) the DMN In-EPs of the patients were negative, whereas those of the controls, positive. This shows that patients' DMN tends to have a non-emulative (nonreplicative) attitude, i.e., all other networks do not tend to copy the DMN activity. For the DAN both groups showed negative in-EPs (non-emulative attitude), but with stronger negative power in ACA patients. The involvement of those networks make sense as seen in the previous paragraph on the clinical relevance. However, the rational for why this novel dynamics metric show stronger discriminatory power as compare to, say, the In or Out-degree of Granger-causality of the DMN and DAN remains unclear. It is indeed difficult to conceive that the replication mechanism between signals do not equate to some degree to a correlation (for sFC) or causal (with GC) effect. In a side study, we actually tried to find similitude between EPs and Deg. GC, which gave us nonsignificant correlation (between the two metric values). A hypothesis is that, since EGN model is based on differential equations, the emulative values between the networks are influencing not by the network signal directly, but rather by their rate of change in activity $(\mathrm{dx}(\mathrm{t}) / \mathrm{dt}$; where $\mathrm{x}(\mathrm{t})=$ RSN activity signal). More details about the EGN model, and its replicator dynamics, in (Madeo et al., 2017).

Regarding SVM and LDA approaches, their ranking in our study is in line with the way these algorithms work. Indeed, in a setup with features having co-variability, and normally distributed, LDA will perform better, with is shown when using many of our most relevant features, and trying to find overall separable (with all of the features) means/centroids. On the contrary SVM, which considers all training data points to find the values (vectors) that help maximising margin between pools of features, is more robust to outliers-which are clearly present in our small dataset. However, we can expect that with a larger multi-dimensional dataset, the two algorithms (LDA and SVM) would not have substantial differences in performance. In order to increase their performances, a solution might be to combine the two algorithms (Alam and Kwon, 2017; Xiong and Cherkassky, 2005). The decision tree algorithm is attractive in its simplicity: sequential splitting of the features with thresholds, but might be too dependent on the training sample-as shown by our strong variance in classification accuracy over the 5 validation methods. Regarding KNN, a more thorough testing with different $\mathrm{K}$ values might have been of benefit. Other type of classifier such as elastic net, logistic regression, or naïve Bayes classifier could possibly be applied as well. However, they have shown in general a substantial lower power at classifying neuropsychiatric disorders, as seen in the summary tables of (Du, Fu, and Calhoun, 2018). Finally, for all classifiers presented here, the Matlab default set-ups of each of the machine learning algorithm were used. We only tried, on our best SVM classifiers, to change the kernel, from Gaussian to polynomial; but this had given us less accurate results. More hyperparameter optimisation could be investigated. Also, the worst-but simplest to explain - algorithm, namely decision tree (best at $80 \%$, using only 6 features, with GC), could further be optimised. For instance, by changing node splitting rules from an ascending impurity gain (of Gini's Diversity Index) to descending one; or by changing directly the predictor selections (which features are used in the nodes of the tree) with curvature tests, or interaction tests.

However, since the results are quite robust in SVM, the first step might be to reproduce the results with new (unseen) data, and see if these classifiers continue to provide high performances. Other neuropsychiatric disorders could also be tested with the same pipeline. E.g. the Autism 
rs-fMRI datasets in the $\mathrm{ABIDE}^{3}$ repository. With such a large dataset, the reliability of the accuracy obtained using our pipeline is much greater, and the optimisation would be more worthy. As mentioned in the introduction, a few fMRI research attempt similar classification of neuropsychiatric disorders using more advanced machine learning algorithms, such as convolutional neural networks, or deep auto-encoder (Meszlényi, Buza, and Vidnyánszky, 2017; Suk et al., 2016). The added value is that those algorithms can be used not only in the supervised multi-labels classification process, but also directly in an unsupervised feature extraction. Though, an important drawback of these advanced classification techniques is that they usually need an important amount of data to be trained upon, which are often not available in case of rs-fMRI data of neuropsychiatric disorders. Also, the understanding and explanation of the feature maps obtained at different CNN layers can be arduous more computationally intensive and troublesome to be clearly explained and descriptive.

Even though research in advance AI for psychoradiology seems promising in many fields, especially segmentation and auto-labelling of cancer or lesion for instance, common machine learning algorithms such as LDA or SVM make the results more understandable and explainable for the clinicians, radiologists and ultimately the patients. For instance, the linear combination of static and dynamic functional connectivity between a handful of well-described networks, can be easily display, and showing how the algorithm split the populations (patients vs controls) is readily feasible. Such simple design and visualisation of the process make possible targetable treatment that must be less complicated to design. And following the evolution of such dynamic (or static) fMRIbased brain features is rather simple, and thus can greatly help to refine classification models to a prognostic level; or to assess (lasting) effects of different treatments of psychiatric disorders.

\subsubsection{Limitations}

There are several limitations in this study. First, as mentioned in the previous paragraph, the study would benefit from a larger dataset. Testing the classifiers on new and unseen data extracted from another scanner (different parameters, e.g. TR or duration of the rs-fMRI), and from another site (different medical centre, or country) would also provide insight regarding the robustness of the methods and the features presented here. However, gathering more fMRI data from ACA patients is not an easy task, since this epilepsy co-morbidity is only recently described, and acquiring systematic rs-fMRI data from elderly patients with epilepsy is not common practise yet. Moreover, having a bigger in-house dataset of participants would not mean getting lower performances, but rather more stable. Indeed, it has been proven by testing pools of data of different sizes, that the accuracy of SVM classification gets simply more reliable (less variability in performances) as the training sample gets bigger, in case of data from schizophrenic patients acquired with the same protocol (Nieuwenhuis et al., 2012). Nevertheless, the cross-centre (i.e. multi-site) robustness still needs to be tested. Second, with high performances of binary classification, we showed a strong diagnostic power of the dynamics features. However, the ACA patients were already diagnosed with ACA based on a clear loss of fluid IQ after psychological assessment. Hence having an automated tool that diagnoses ACA with lower accuracy than diagnoses based on psychological tests are not needed. In that regards, our goal was not to show the highest performance, but to rank the dynamics features, and show the benefit of combining different dynamic features, using solely rsfMRI data. Also, with refinement, this analysis could extrapolate from the two class (binary) classification/diagnosis to a multi-class separation, and show prognostic power, for example in order to predict mental ages, or rate of decline in intelligence of patients with ACA (based on a stratified approach, through severity of the ACA for instance). Despite those limitations, we are confident

\footnotetext{
${ }^{3}$ http://fcon_1000.projects.nitrc.org/indi/abide/
} 
that the emulative powers, and the times of in-phase coherence of large-scale resting-state networks are informative descriptors of neuropsychiatric disorders, and that combining them with known metrics such as SFC and GC can lead to accurate diagnoses. One could expect similar performance, when testing the new metrics upon schizophrenic, autistic, or depressive patients for example. And this pipeline to train data and validate clinical diagnoses of neuropsychiatric disorders can be of great help in clinics and contribute to the field of psychoradiology (Lui et al., 2016).

\subsection{Conclusion}

We presented a novel metric for neurodynamics extracted from the evolutionary game theory on network approach: The emulative power of brain networks, i.e. the propensity of a network for replicating another network activity. The emulative powers of the default mode network and dorsal attention network seem defective in ACA; and using these features combined with inphase coherence measurements, and static functional connectivity yield to an average diagnostic accuracy of $90 \%$, using the SVM algorithm. With regard to the field of neuropsychoradiology, other neuropsychiatric disorders could be tested using similar fMRI-based dynamics features, and could lead to better understanding, and subsequently better treatments of mental disorders. Also, with refinements of the models and the classification pipeline, we could envisage that the fMRIbased neurodynamics presented here would have a prognostic value, for example if labels are not simply binary (stratified models), other feature types are added (multimodal approaches), and using cohort study design (longitudinal cross-sectional studies). This pipeline applied on larger datasets, and if replicated, would demonstrate robustness of these new neurodynamics metrics and reliability of the methods, and hence could be to some extent clinically used. 

Chapter 7

\section{A Bayesian method for inference of effective connectivity in brain networks for detecting the Mozart effect}

[Submitted] 


\begin{abstract}
Several studies have claimed listening to Mozart's Sonata K448 has particular effects on cognition and neurological conditions, for example epilepsy, but no well-established theories exist on the neural dynamics in the brain that might facilitate this Mozart effect. Research into this Mozart effect has not addressed how dynamic interactions between brain networks, i.e. effective connectivity, might be affected by Mozart's music.

In this paper we will focus on how effective connectivity between brain networks, measured through fMRI BOLD measurements, is affected by Mozart music. Granger-causality analysis is often used to infer effective connectivity using fMRI data. In this paper we will first investigate if a new method, Bayesian topology identification, can be used as an alternative for the inference of effective connectivity. The performance of both methods in simulation is evaluated, where the Bayesian method outperforms the Granger causality analysis in inference of the ground truth connectivity of simulated dynamic networks, especially for short data lengths. Furthermore, an extension is developed for the Bayesian method to enable it to draw conclusions on differences in connectivity between groups of subjects. Next, the influence of Mozart's sonata K448 on the brain network connectivity through fMRI data of 16 healthy adults is investigated, using both the extended Bayesian topology identification and Granger-causality analysis.

Using the extended Bayesian method, a significant effect was found for subjects with a long listening duration in the connection from the posterior default mode network to the fronto-parietal right network, which is related to cognitive processing. Furthermore, a significant change was found for subjects with a shorter listening duration in the connection from the sensori-motor lateral network to the superior temporal gyrus, which is related to auditory processing in the brain.
\end{abstract}




\subsection{Introduction}

In the last decade the effects of music on the brain have been an active topic of research (Blood et al., 1999; Koelsch et al., 2005; "Singing in the brain: Neural representation of music and voice as revealed by $\left.\mathrm{fMRI}{ }^{\prime \prime}\right)$. In particular one piece of classical music, Mozart's Sonata K448, has been claimed to have unique effects on the brain, besides the general effects caused by music. One of the original studies by Rauscher et al. (Rauscher, Shaw, and Ky, 1993) reports that listening to Mozart music increases spatial and temporal reasoning skills in healthy subjects, but this effect did not last beyond the 15 minute testing period. However, a strong effect specific to Mozart music was unable to be replicated in repeated studies (Pietschnig, Voracek, and Formann, 2010). In another study (Verrusio et al., 2015) neural activity was measured through spectral analysis of electroencephalogram (EEG) data. After listening to Mozart music, an increase in brain wave activity linked to memory, cognition and problem solving was observed in adults.

Besides these effects, Mozart music has also been reported as being effective as a medicine. First of all, in (Coppola et al., 2015) it is reported that consistent exposure to Mozart music for 15 days caused a reduction in the frequency of epileptic seizures in children with epilepsy and in some cases led to complete recession of seizures. Furthermore, the effects persisted even after the exposure to Mozart music was stopped. Another study (Bodner et al., 2012) reported similar positive results in adults with epilepsy. Finally, in a study of the influence of Mozart music therapy on schizophrenia patients (Yang et al., 2018), Mozart music was found to have effects on the functional connectivity of patients, which was inferred from functional magnetic resonance imaging (fMRI) data.

However, no well-established theories exist on the neural dynamics in the brain that are responsible for the Mozart effect found in theses studies. Moreover, research into the existence of a Mozart effect does not focus on inferring effective connectivity between brain networks from time series, so in this work we will focus on methods that can infer how brain networks are connected and interact, using fMRI BOLD time series that describe the dynamic neural activity from those brain networks.

The brain is a dynamic system; cognition and consciousness arises from not just the static correlations between brain networks, i.e. functional connectivity, but also through the dynamic behaviour of the brain. Therefore, the inference of dynamic interactions of brain networks, i.e. effective connectivity, is an important aspect in the analysis of the brain as it reveals how brain networks dynamically influence each other. By far the most popular method of inference of effective connectivity is the Granger-causality analysis (Geweke, 1984; Granger, 1969). While the method originated in the field of economics, it has been used in the past for the inference of brain connectivity using EEG Barrett et al., 2012 and fMRI data of the brain (Bernas et al., 2018). In this paper we focus on the inference of effective connectivity using resting-state fMRI BOLD measurements. We could infer connectivity directly from voxel BOLD measurements (Beer et al., 2019) or from the mean BOLD signals in brain networks defined by an atlas (Rosa et al., 2015). Instead, we will follow an approach that uses independent component analysis (ICA) (Beckmann et al., 2009; Hyvärinen, Karhunen, and Oja, 2001) to find active brain networks and their corresponding ICA time series. These ICA time series are then used as an indication of dynamic behaviour of that brain region (Bernas et al., 2018; Demirci et al., 2009; Londei et al., 2006; Regner et al., 2016). An advantage of the use of ICA time series in combination with methods like Granger-causality analysis is that the inference does not rely on any prior hypotheses on the locations of neural activity.

Besides Granger-causality analysis, several other methods have been developed to infer the effective connectivity. First, there are several methods which use a parametric network model to infer 
effective connectivity (Friston, Harrison, and Penny, 2003; Wang, Sznaier, and Camps, 2016). The first approach, DCM (Friston, Harrison, and Penny, 2003) is specifically designed to model neural activity (Buxton, Wong, and Frank, 1998) and effective connectivity using specialised state-space models, for example in (Marreiros, Kiebel, and Friston, 2008) excitatory and inhibitory states are used, which is in line with how populations of neurons function in the brain. While the specialised models are useful to model brain activity more accurately, the estimation of the model is computationally expensive and DCM is therefore not often used as an exploratory method to assess effective connectivity between brain networks. The methods in (Beer et al., 2019; Wang, Sznaier, and Camps, 2016) use a regression model with a re-weighted regularization to force a subset of model parameters of a connection to zero in an effort to infer a sparse estimate of the effective connectivity. While this method scales very well with large dynamic networks, i.e. with many nodes, it has tuning parameters which must be carefully chosen to achieve a good estimate of the effective connectivity.

Furthermore, there exist non-parametric approaches, such as the method detailed in (Materassi and Salapaka, 2012). This method calculates a Wiener filter estimate of the dynamics of one connection to infer if the connection exists in the dynamic network or not. However, this approach requires a lot of data to compute accurate estimates of the effective connectivity.

In this paper, we will consider a relatively new method, Bayesian topology identification (Chiuso and Pillonetto, 2012; Shi, Bottegal, and Hof, 2019), which employs a Bayesian model selection approach to estimate the connectivity of a dynamic network. Through the incorporation of prior knowledge this method performs well even when not a lot of data is available. However, it does not scale very well with large networks. This makes Bayesian topology identification especially useful in combination with the ICA procedure, as the use of ICA reduces the high spatial dimensionality of the voxel data in fMRI scans to a much lower dimension in the obtained ICA time series.

Our first research question is to investigate whether Bayesian topology identification can be used as an alternative to Granger-causality analysis for the inference of the effective connectivity using fMRI data. First, we investigate if there is a significant difference in the performances of the two methods in a simulation setup. Then, because the Bayesian method was originally developed to infer graph estimates of the brain network connectivity of one individual, we extend the Bayesian method such that we can draw conclusions on the connectivity of groups of data sets, which is beneficial in the inference of the Mozart effect.

After we have extended the Bayesian approach, our second research question is to investigate the influence of listening to Mozart's Sonata K448 on effective connectivity through fMRI data of healthy adults. Both the Granger-causality analysis and the Bayesian approach are applied in the Mozart effect study, to consolidate the findings. Here, we put more emphasis on the results obtained from the approach which performs better in the simulation study. Furthermore, we will investigate if the length of time that subjects listened to Mozart music leads to different conclusions on the effective connectivity.

This paper is structured as follows: first, we will detail the model we will use to describe the dynamic interactions between brain regions. Second, we describe the existing Granger-causality analysis and how we can infer connectivity using fMRI data. Third, we detail the Bayesian topology identification method and develop an extension to the Bayesian method that can test the hypothesis of effective connectivity change between two groups of subjects. Then, we test the performance of the Granger-causality analysis and the Bayesian method using simulated data. Finally, we will apply the two methods to the real fMRI data and infer if listening to Mozart's Sonata K448 has an effect on the effective connectivity of the subjects in the study. 


\subsection{Theory}

To infer brain network connectivity using fMRI measurements of the neurodynamics of the brain, we will first define a modelling framework wherein the dynamic interactions between brain networks can be modelled appropriately.

\subsubsection{Dynamic network model of brain network connectivity}

The effective connectivity between brain networks, using fMRI measurements to describe neural activity, can be described by linear models (Seth, Chorley, and Barnett, 2012). Furthermore, in general the causal relationship between two brain networks can be described by an infinite impulse response (IIR) filter, as long as the hemodynamics Ogawa et al., 1990 can be considered as an invertible filter (Seth, Chorley, and Barnett, 2012). The neural activity of brain networks is described by $L$ time series $w_{j}(t), j=1, \ldots, L$, i.e. one ICA time series for each brain network found by an ICA decomposition of fMRI measurements of a subject. Now, the dynamic interactions between brain networks can be modeled as follows (Hof et al., 2013):

$$
\begin{aligned}
& w_{j}(t)=\sum_{i \in I} G_{j i}(q) w_{i}(t)+\eta_{j}(t), \quad j \in I, \\
& G_{j i}(q)=\sum_{k=1}^{\infty} \theta_{j i, k} q^{-k},
\end{aligned}
$$

where $q$ is the shift operator, i.e. $q^{-1} w_{j}(t)=w_{j}(t-1), I=\{1, \ldots, L\}, \theta_{j i, k}$ indicates one coefficient of $G_{j i}(q)$ and $\eta_{j}(t)$ denotes the background noise of brain network $j$. The full model is written in matrix form as:

$$
w(t)=G(q) w(t)+\eta(t)
$$

where $G(q)$ is a $L \times L$ matrix, where entry $(j, i)$ is $G_{j i}(q), w(t)=\left[w_{1}(t), \ldots, w_{L}(t)\right]^{T}$ and $\eta(t)=$ $\left[\eta_{1}(t), \ldots, \eta_{L}(t)\right]^{T}$.

Some assumptions are made on the components of the model in (7.1):

- $G_{j i}(q)$ is stable for all $j, i$, i.e. $\sum_{k=1}^{\infty}\left|\theta_{j i, k}\right|<\infty$;

- $(I-G(q))^{-1}$ is stable, i.e. all its entries are stable;

- $\eta_{j}(t)$ is Gaussian distributed with zero mean and unknown variance $\sigma_{j}^{2}$, and is independent over $j$ and $t$.

Each IIR transfer operator $G_{j i}(q)$ can be approximated by a finite-order impulse response of order $m$, which will have no significant effect on the performance of the model if $m$ is chosen large enough. The approximation is written as an auto-regressive (AR) model of order $m$ :

$$
w_{j}(t)=\sum_{i=1}^{L} \sum_{k=1}^{m} w_{i}(t-k) \theta_{j i, k}+\eta_{j}(t)
$$

and written in matrix form:

$$
w_{j}=\sum_{i \in I} A_{j i} \theta_{j i}+\eta_{j}
$$




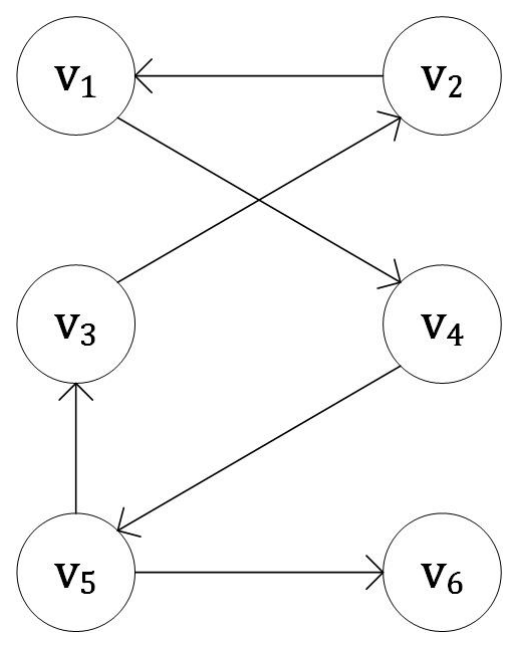

FIGURE 7.1: Example of a six-node directed graph.

where

$$
A_{j i}=\left[\begin{array}{cccc}
w_{i}(-1) & w_{i}(-2) & \ldots & w_{i}(-m) \\
w_{i}(0) & w_{i}(-1) & \ldots & w_{i}(-m+1) \\
\vdots & \ddots & \ddots & \vdots \\
w_{i}(N-1) & w_{i}(N-2) & \ldots & w_{i}(N-m)
\end{array}\right]
$$

Here $w_{j} \in \mathbb{R}^{N}, \eta_{j} \in \mathbb{R}^{N}$ are vectors of time series of brain network $j, \theta_{j i}$ is the parameter vector of the connection from $w_{i}(t)$ to $w_{j}(t)$, and $A_{j i}$ is the matrix of time shifted columns of $w_{i}$, where $w_{i}(t)=0$ for $t<0$. This VAR model will be used in the inference of Granger-causality and furthermore forms the modelling framework on which the Bayesian topology identification method relies.

\subsubsection{Directed graph}

We can also define a graphic representation of model (7.1) that encodes the effective connectivity between brain networks. A directed graph of model (7.1) is denoted as $\mathcal{G}=(V, E)$, where $V$ is the set of all nodes and $E$ is the set of all directed edges in the graph. Node $v_{j} \in V$ in the graph represents a particular brain network, defined by the ICA procedure, and its dynamic behaviour is described by the corresponding ICA times series $w_{j}(t)$. Thus the cardinality of $V$ equals the total number of ICA time series. A directed edge from $v_{i}$ to $v_{j}$ exists, denoted as $e_{j i} \in E$ or $e_{j i} \in \mathcal{G}$, if and only if $G_{j i}$ in (7.1), or equivalently, $\theta_{j i}$ in (7.4) is non-zero, which indicates a causal connection from node $v_{i}$ to $v_{j}$. We also denote the set of all incoming edges to $v_{j}$ as $E_{j}$ or $\mathcal{G}_{j}$. Now, a graph $\mathcal{G}$ indicates the effective connectivity between brain networks. An example of such a directed graph is shown in Figure 7.1.

\subsection{Materials}

\subsubsection{Simulation data generation}

Before being applied to the real fMRI data, which typically suffers from low sampling rates and measurement noise, the methods for the inference of the effective connectivity should at least show a reasonable performance for the simulated data generated in an ideal setting, i.e. without 


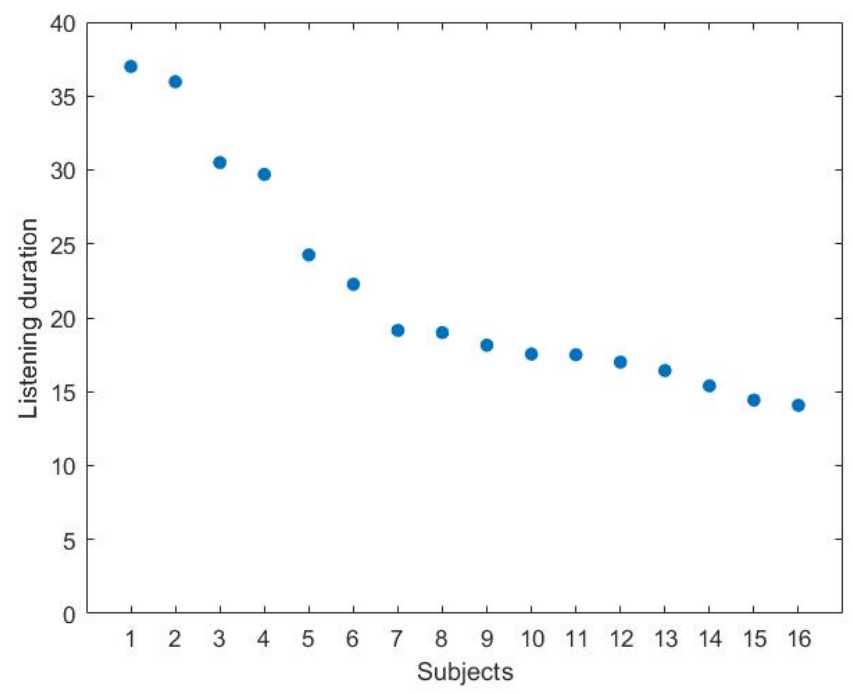

FIGURE 7.2: Listening duration in hours of all 16 subjects ordered from the longest to shortest listening subject.

the above complexities. Since the data generation model is known in simulation, the goal of the simulation study is to test how well the estimated connectivity of one method approximates the true connectivity.

In this work, in order to evaluate the relative performances of the Granger-causality analysis and the Bayesian topology identification, we test them in a controlled environment using the simulated data, which is generated by models as defined in (7.1). To be precise, we will use the same method for data generation as performed in (Shi, Bottegal, and Hof, 2019). This model type is generally a good approximation of the BOLD signal caused by the neural activity, while the values of coefficients are generated randomly over a general range, which also covers the hemodynamics and the neural activity of brain networks as special cases.

The node time series $w_{j}(t)$ are generated from a model (7.1), where some random directed edges $e_{j i}$ are chosen to form a ground truth connectivity $\mathcal{G}_{0}$, and for each $e_{j i}$ which exists in $\mathcal{G}_{0}$, its corresponding $G_{j i}(q)$ is a random transfer function (Hof et al., 2013), such that the resulting dynamic network adheres to the IIR model assumptions. The noise $\eta_{j}(t)$ in (7.1) is randomly sampled from a Gaussian distribution for each node in the simulation model.

\subsubsection{Mozart effect study}

Sixteen volunteers ( 11 female, 5 male) without a medical history of neurological or psychiatric disease, aged 20-65, participated in the experiment. To prevent bias, subjects with a variety of taste in music were selected and no subjects were selected who already consistently ( 2 hours or more per day) listened to Mozart music. Informed consent of the experiment and scans were obtained from each subject. The scanning of healthy volunteers was approved by the Medical Ethical Committee of the Academic Centre for Epilepsy Kempenhaeghe (Heeze, The Netherlands).

\section{Mozart's Sonata K448 experiment}

Four fMRI scans are collected for each subject in the study. Each of the four scans are taken one week apart in time. In the week between scan 1 and 2, subjects were instructed not to listen to 
TABLE 7.1: List of all brain networks found in the group ICA decomposition.

\begin{tabular}{|l|l|l|}
\hline$\#$ & Shorthand & Description \\
\hline 1 & DMN_ANT & default mode network, anterior \\
\hline 2 & MED_VISU & medial visual cortex \\
\hline 3 & OCC_LAT_VISU & occipetal \& lateral visual cortex \\
\hline 4 & DAN & dorsal attention network \\
\hline 5 & FPR & fronto-parietal right network \\
\hline 6 & SM_LAT_AUDI & sensori-motor, lateral network \\
\hline 7 & FPL & fronto-parietal left network \\
\hline 8 & VAN & ventral attention network \\
\hline 9 & LING_FUS & lingual fusiform cortex \\
\hline 10 & DMN_POS & default mode network, posterior \\
\hline 11 & N11 & noise \\
\hline 12 & SAL_AUDI & salience auditory network \\
\hline 13 & ANG & angular gyrus \\
\hline 14 & N14 & White matter \\
\hline 15 & SUPTEMP & superior temporal gyrus \\
\hline 16 & CEN & central executive network \\
\hline 17 & N17 & noise \\
\hline 18 & SM_SUP & sensori motor medial superior \\
\hline 19 & CEREB & cerebellum \\
\hline 20 & N20 & borders and movements \\
\hline
\end{tabular}

Mozart's Sonata K448 for any amount of time. Then, the subjects were instructed to listen for a minimum of 14 hours to Mozart's Sonata K448 during the week between scan 2 and 3 and to listen for at least 2 hours each day unless this was not possible due to unavoidable circumstances. The listening duration of each subject during the week of music exposure was recorded by the subjects themselves. In Figure 7.2 we can see the spread of total listening duration across all 16 subjects. After the third scan subjects were once again instructed to stop listening to Mozart music and then the final scan is taken one week after the subjects stopped listening to Mozart's Sonata K448. From the first scan to the fourth scan, subjects were also instructed to avoid listening to any other music, but limited exposure to music in public places could in some cases not be avoided.

\section{Acquisition}

Imaging was performed using a 3T Philips Achieva MRI scanner. A T1-weighted reference scan was recorded using a 3D spoiled gradient-echo sequence (T1TFE), TR/TE: $8.3 / 3.5 \mathrm{~ms}$. The matrix size was $240 \times 240 \times 180$ with isotropic voxels of $1 \mathrm{~mm}^{3}$. Total T1 scan duration was 600s. Second, a multi-echo time fMRI scan was recorded using a gradientecho EPI sequence with TR/TE/ES: 2000/12/23ms. 26 slices with a width of $4.5 \mathrm{~mm}$ (0mm gap) were recorded. The data were acquired with $3.5 \times 3.5 \mathrm{~mm}$ in-plane resolution, final in-plane resolution was also $3.5 \times 3.5 \mathrm{~mm}(64 \times 64)$. SENSE (Pruessmann et al., 1999) acceleration was used with SENSE factor of 2.7 in the read-out direction. The total multi-echo scan time was $608 \mathrm{~s}$.

During all scans the subjects were asked to remain still with their eyes open and to focus on a projected dark-blue cross. Physiology data (heart and respiratory rate) were recorded using a standard scanner set-up. 

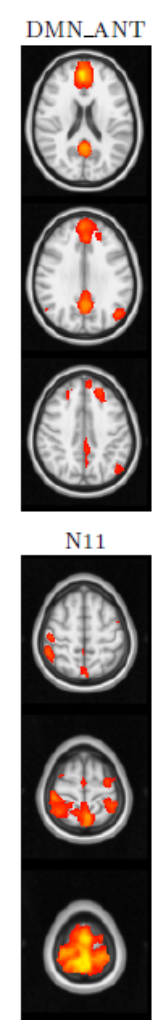
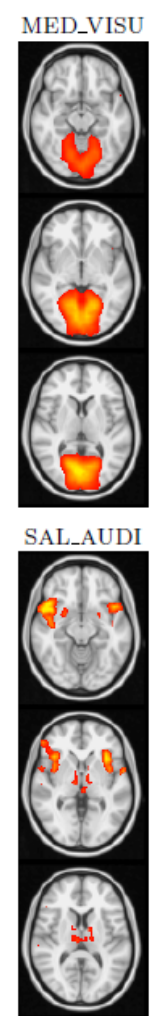
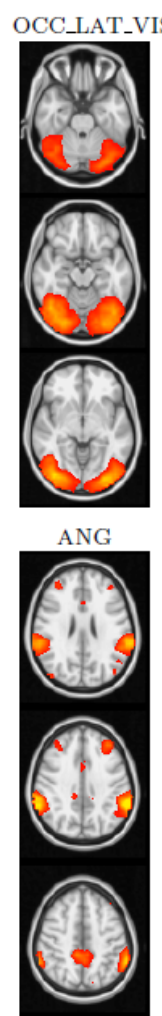

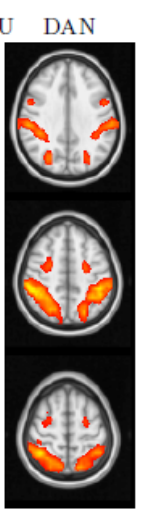

$\mathrm{N} 14$

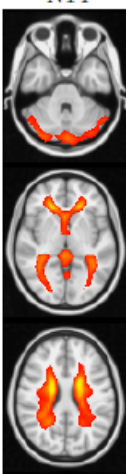

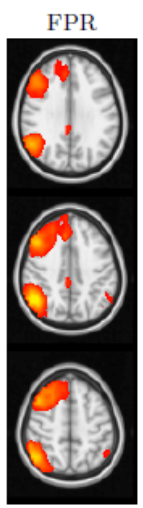

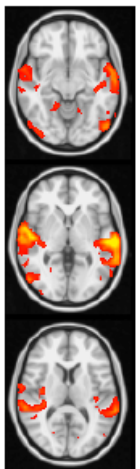

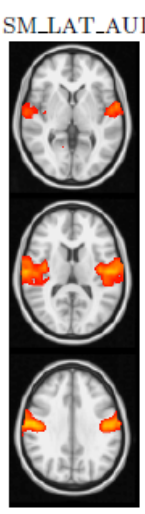

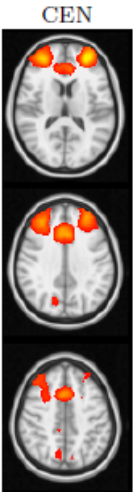

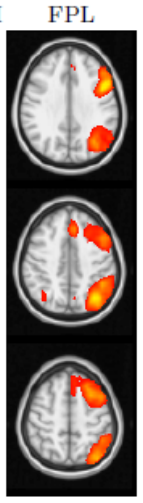

$\mathrm{N} 17$

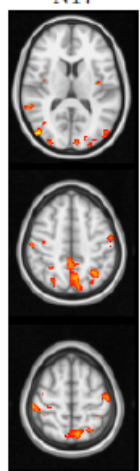

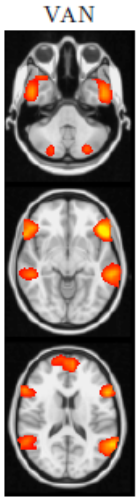

SM_SUP

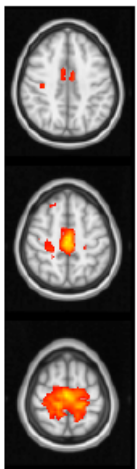

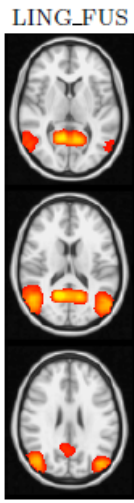
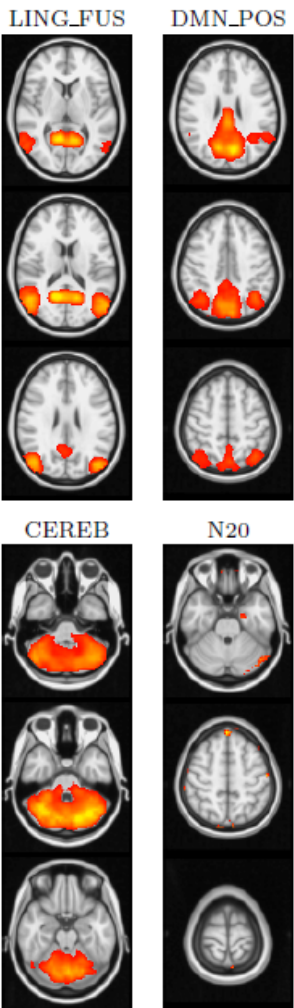

FIGURE 7.3: Spatial maps of the 20 active brain networks found through the ICA decomposition. Each image consists of 3 relevant horizontal slices of the brain, where the spatial map is indicated by the red colour scale.

\section{Preprocessing}

First, non-BOLD signals caused by physiological effects such as movement, breathing and pulse were removed by multi-echo ICA (MEICA; Kundu et al., 2012). Then, using FSL (FMRIB Software Library v5.0; Beckmann et al., 2009) all 64 data sets were temporally concatenated and a group ICA was performed to identify 20 independent components, which are listed in Table 7.1. The spatial maps of these brain networks can be found in Figure 7.3. Next, dual regression (Beckmann et al., 2009) was performed to obtain individual spatial maps and time series for each fMRI scan of the subjects, where each ICA time series consists of $N=300$ data points.

\subsection{Methods}

This section is divided in two parts. In the first part, we briefly discuss both the Granger-causality analysis and the Bayesian topology identification. Next, we detail how we apply both methods to the simulation data. Then, we develop the extension to the Bayesian method. In the second part, we first validate the use of AR models to model the ICA time series. Then we will apply the extended Bayesian topology identification and the Granger-causality analysis to the ICA time series of the Mozart effect study. 


\subsubsection{Granger-causality analysis}

Here we briefly describe the well-established Granger-causality analysis, which will be used as a reference to compare to the Bayesian topology identification. The Granger-causality analysis is based on the concept that if for a given $j$ and $i, \theta_{j i}$ in (7.4) is significantly non-zero, then setting parameter vector $\theta_{j i}$ to 0 will significantly increase the size of the residual $\eta_{j}(t)$. However to relate this change in the residual to a real connection between two brain networks and not from a shared influence from other regions, we will infer connectivity only using the conditional Grangercausality analysis.

existence of a connection $e_{j i}$ is inferred by fitting two different AR models to the measurement data and comparing the residuals. The first AR model includes all $L$ node time series in the regression as in (7.4). In the second AR model $A_{j i}$ corresponding to connection $e_{j i}$ is excluded:

$$
w_{j}=\sum_{k \in I \backslash i} A_{j k} \cdot \theta_{j k}^{\prime}+\eta_{j}^{\prime} .
$$

The model order $m$, i.e. the length of parameter vectors $\theta_{j i}$, is selected through the Akaike information criterion (AIC) (Akaike, 1974), to sufficiently model the interactions in the data without overfitting, as it can make the Granger-causality analysis unreliable (Barnett and Seth, 2014).

Next, the Granger-value $\mathcal{F}_{j i}$ corresponding to connection $e_{j i}$ is defined as the logarithm of the ratio of the variances of the residuals:

$$
\mathcal{F}_{j i}=\log \frac{\operatorname{var}\left[\eta_{j}^{\prime}\right]}{\operatorname{var}\left[\eta_{j}\right]} .
$$

The Granger-value $\mathcal{F}_{j i}$ is the degree to which the addition of connection $e_{j i}$ helps predict $w_{j}$ and as such, a significantly non-zero $\mathcal{F}_{j i}$ indicates the existence of connection $e_{j i}$. Furthermore, $\mathcal{F}_{j i}$ asymptotically follows a $\chi^{2}$ distribution (Barnett and Seth, 2014) as data length $N \rightarrow \infty$ and thus we can perform a statistical test (Barnett and Seth, 2014) to infer if $\mathcal{F}_{j i}$ is significantly non-zero. Furthermore, the Granger-value is equivalent to the transfer entropy (Barnett, Barrett, and Seth, 2009; Kaiser and Schreiber, 2002; Schreiber, 2000), a measurement of the rate of information transfer between two time series, which is an information-theoretic concept. As such, $\mathcal{F}_{j i}$ can be interpreted as a measurement of connectivity strength. Therefore, a change in Granger-value of a connection between two fMRI scans of a subject indicates a difference in the connectivity strength of that connection.

However, it is important to note that, when inferring effective connectivity using ICA time series from fMRI measurements, it is ill-advised to infer a graph estimate of the connectivity, as prior research (Barnett and Seth, 2014; Seth, Chorley, and Barnett, 2012) indicates that the $\chi^{2}$ statistical test becomes unreliable through an overall increase in false positives. Nevertheless, the equivalence of Granger-causality with the transfer-entropy remains valid and therefore we can use a paired $\mathrm{t}$-test to test for differences in connectivity strength between two fMRI scans.

For the simulation study, since the effective connectivity of the data-generation systems is known, our goal is to quantify the quality of $\mathcal{F}_{j i}$ as a measurement of connectivity. If we want to be able to compare the quality of the estimates of both methods on simulated data, we should use the $\chi^{2}$-test to determine a graph estimate, as the Bayesian method also infers a graph estimate. If we would use the paired t-test on the simulation data, it would make comparing the estimation performance between the methods impossible. Even though this is not the method we will use on the real data, it still gives us an indication of the reliability of $\mathcal{F}_{j i}$ as a measurement of connectivity. As such, we will infer an estimate $\hat{\mathcal{G}}$ of the ground truth connectivity $\mathcal{G}_{0}$ by testing the significance of $\mathcal{F}_{j i}$ for all connections $e_{j i}$ and adding the connection to $\hat{\mathcal{G}}$ if the statistical test is significant. To calculate the 
reduced regression in (7.5), the Granger-values in (7.6) and the significance of the Granger-values, we will use the MVGC MATLAB toolbox (Barnett and Seth, 2014).

\subsubsection{Bayesian topology identification}

Bayesian topology identification (Chiuso and Pillonetto, 2012; Shi, Bottegal, and Hof, 2019) is a Bayesian machine learning method that infers from node time series data $D=\left\{w_{1}, \ldots, w_{L}\right\}$ an estimate $\hat{\mathcal{G}}$ of $\mathcal{G}_{0}$, the connectivity of the dynamic network. A Bayesian model selection (Bishop, 2009) approach is used to compare the posterior probability $p(\mathcal{G} \mid D)$ of different graphs and then selects the graph with maximum $p(\mathcal{G} \mid D)$ as the optimum graph estimate $\hat{\mathcal{G}}$. Given a graph, the posterior probability $p(\mathcal{G} \mid D)$ can be calculated using Bayes' rule:

$$
p(\mathcal{G} \mid D)=\frac{p(D \mid \mathcal{G}) p(\mathcal{G})}{p(D)} .
$$

The evidence $p(D)$ can be difficult to calculate, as it requires a marginalization over the set of all graphs. Instead, under the assumption that the prior distribution of graphs is uninformative, i.e. $p(\mathcal{G})$ is constant, the graph with the maximum marginal likelihood $p(D \mid \mathcal{G})$ will also have the maximum posterior odds $p(\mathcal{G} \mid D)$. As such, the graph estimate $\hat{\mathcal{G}}$ is selected as the graph with the maximum marginal likelihood.

The marginal likelihood $p(D \mid \mathcal{G})$ can be computed through marginalization over its parameters $\theta$ :

$$
p(D \mid \mathcal{G})=\int p(D \mid \theta, \mathcal{G}) p(\theta \mid \mathcal{G}) d \theta
$$

where $p(D \mid \theta, \mathcal{G})$ is the likelihood of model parameters $\theta$ and a graph $\mathcal{G}$ and $p(\theta \mid \mathcal{G})$ is the prior distribution of $\theta$ given a graph $\mathcal{G}$.

In the context of this study, the model parameters $\theta$ in the parameter prior $p(\theta \mid \mathcal{G})$ indicate the set of all $\theta_{j i}$ of an AR model as defined in (7.4), where parameter vectors $\theta_{j i}$ are modelled as Gaussian random vectors. The co-variance matrices of these random vectors are parametrised with some hyperparameters, which encode the assumption of stability. The hyperparameters of random parameter vectors $\theta_{j i}$ and the variance $\sigma_{j}^{2}$ of $\eta_{j}$ are estimated using an Expectation-Maximization (EM) algorithm (Bishop, 2009) as described in (Shi, Bottegal, and Hof, 2019). Because the $\theta_{j i}$ are modeled as random vectors, we can simply set model order $m$ in (7.4) to some large number at most equal to the data length $N$, instead of determining an optimal model order, as the estimated hyperparameters will determine the relevancy of each of the $m$ parameters.

Now, under the assumption of Gaussian distributed noise, $p(D \mid \theta, \mathcal{G})$ can be computed based on (7.4):

$$
p(D \mid \theta, \mathcal{G})=\prod_{j=1}^{L} \mathcal{N}\left(\sum_{i \in C} A_{j i} \theta_{j i}, \sigma_{j}^{2} I_{N}\right),
$$

where $C=k \mid e_{j k} \in \mathcal{G}$. Given this parametrization, the integral in (7.8) has a closed form solution (Shi, Bottegal, and Hof, 2019) and thus we can avoid a numerical integration, which could be computationally costly.

The graph $\mathcal{G}$ with the maximum marginal likelihood $p(D \mid \mathcal{G})$ is chosen as the connectivity estimate $\hat{\mathcal{G}}$. This maximum can be found by comparison of the marginal likelihoods of all possible graphs, which is computationally expensive when the number of nodes in the network is large. Therefore, to avoid the combinatorial problem of comparison of all possible graphs, a greedy search algorithm (Chickering, 2002; Shi, Bottegal, and Hof, 2019) is employed that efficiently finds a graph estimate $\hat{\mathcal{G}}$. 


\subsubsection{Evaluation of methods in simulation}

To motivate the use of the Bayesian topology identification for the inference of brain network connectivity, we will evaluate whether the Bayesian approach has any advantages over Grangercausality analysis in the estimation of the connectivity using simulation data. Now, recall that the true effective connectivity of the simulation model $\mathcal{G}_{0}$ is known. The graph estimates $\hat{\mathcal{G}}$ of both methods are compared to $\mathcal{G}_{0}$ and the quality of the estimate is quantified using the true positive rate (TPR) and the false positive rate (FPR):

$$
\begin{aligned}
& \mathrm{TPR}=\mathrm{TP} / \mathrm{P}, \\
& \mathrm{FPR}=\mathrm{FP} / \mathrm{F},
\end{aligned}
$$

where the TPR is the number of edges in $\hat{\mathcal{G}}$ that also exist in $\mathcal{G}_{0}$, denoted as TP, over the total number of edges in $\mathcal{G}_{0}$, denoted as P. The FPR is the number of edges in $\hat{\mathcal{G}}$ that do not exist in $\mathcal{G}_{0}$, denoted as FP, divided by the total number of edges that do not exist in $\mathcal{G}_{0}$, denoted as F. To avoid uncertainties in the conclusion on the relative performance of the two methods given certain design parameters for the simulation, i.e. a particular data length $N$ and certain network size in terms of number of nodes, 50 random simulation models with random $\mathcal{G}_{0}$ are generated, and then TPR and FPR are calculated by averaging the results of the 50 estimates.

The design parameters for the simulation are chosen as follows. To test how the performance of both methods varies with the data length, we consider three different settings with $N=$ $\{50,300,2000\}$ for six-node dynamic networks. Furthermore, to evaluate how the number of nodes in the network affects the estimation performance, we compare the TPR and FPR of the methods between six and twelve node networks, with $N=2000$. Finally, for each of the previous evaluations we will assess the performance of the Granger-causality analysis over a range of thresholds, determined by a range of significance levels $p=\{0.005,0.05, \ldots, 0.95\}$ of the $\chi^{2}$ test, which are corrected for multiple comparisons using the false discovery rate (FDR) correction (Hochberg and Tamhane, 1987) in the MVGC MATLAB toolbox. This latter evaluation is performed to rule out differences in the performance of Granger-causality analysis and the Bayesian topology identification caused by a specific choice of significance threshold.

\subsubsection{Bayesian group hypothesis test}

The Bayesian topology identification method is designed to obtain a graph estimate of the effective connectivity from a single fMRI scan, which can be used in simulation to compare with the true connectivity. However, it is not directly applicable to the Mozart effect study using data sets from groups of subjects. In the Mozart effect study there are 4 groups of data sets of subjects, where each group refers to one of the four measurement moments in the four consecutive weeks. In this latter study, we want to infer how the overall brain network connectivity of the subjects changes over the weeks. Using the Bayesian method, changes in effective connectivity can only be measured through a change in the presence of a connection in the graphs of the subjects. For a group of subjects we define for each connection separately the following hypotheses:

$H_{1}$ : Connection $e_{j i}$ is present in the group.

$H_{0}$ : Connection $e_{j i}$ is absent in the group.

To infer if changes in the overall brain network connectivity occurred over the four weeks, we first find the most likely hypothesis for each week separately and then the optimal hypothesis of change is the collection of the most likely hypotheses of each week. If the group hypothesis changes between the weekly scans, this indicates an overall change in the effective connectivity of this connection. 
A preliminary way to asses which hypothesis might be more likely than the other for each week, is the Bayesian selection frequency of the connection, i.e. the number of subjects whose graph estimate includes this connection. It will allow us to choose connections in the Mozart effect study which might be of interested, which is useful because evaluating the optimal hypotheses of all connections can be computationally expensive. If the selection frequency for a given week is close to 0 , we expect the most likely hypothesis to be $H_{0}$, if the selection frequency is closer to 16 , i.e. the connection was present in almost all the subjects, we expect the most likely hypothesis will be $H_{1}$. The Bayesian selection frequency can be easily reported for every connection from the graph estimates of each subject over the four weeks.

Now, to actually evaluate which of the two hypotheses is actually more likely than the other for a given connection in one week, we will calculate the likelihood of the two hypotheses:

$$
\begin{aligned}
& p\left(\mathcal{D} \mid H_{1}\right)=\prod_{k \in S} p\left(D^{k} \mid e_{j i}\right), \\
& p\left(\mathcal{D} \mid H_{0}\right)=\prod_{k \in S} p\left(D^{k} \mid \neg e_{j i}\right),
\end{aligned}
$$

where with some abuse of notation $e_{j i}$ indicates the existence of the connection, $D^{k}$ indicates the data from subject $k, S$ is the index set of all subjects and $\mathcal{D}$ is defined as the collection of data $D^{k}$ from each subject in the group. The likelihoods $p\left(D^{k} \mid e_{j i}\right), p\left(D^{k} \mid \neg e_{j i}\right)$ in (7.12) of each subject are not yet known and must be calculated from the marginal likelihood in (7.8), which is calculated by the Bayesian method. The likelihoods in (7.12) can be calculated through the marginalization of $p(D \mid \mathcal{G})$ over all graphs for which the hypothesis is true. As an example we use hypothesis $H_{1}$ here, but the calculation is similar for $H_{0}$ :

$$
p\left(D^{k} \mid e_{j i}\right)=\sum_{\mathcal{G} \in \mathcal{P}_{1}} p(\mathcal{G}) p\left(D^{k} \mid \mathcal{G}\right),
$$

where

$$
\begin{aligned}
& \mathcal{P}_{1}=\left\{\mathcal{G} \mid e_{j i} \in \mathcal{G}\right\}, \\
& \mathcal{P}_{0}=\left\{\mathcal{G} \mid e_{j i} \notin \mathcal{G}\right\} .
\end{aligned}
$$

This marginalization over graphs can be seen as averaging out the effects that other connections have on the likelihood of the connection in the hypothesis. The calculation of $p\left(D^{k} \mid e_{j i}\right)$ in (7.13) can become computationally expensive for large graphs, therefore in $\mathrm{D}$ we detail how to simplify calculation of this marginalization.

Now, we can calculate the hypothesis likelihoods in (7.12) and we can find the optimal hypothesis by comparison of the likelihoods of the two hypotheses:

$$
\mathrm{BF}=2 \log \frac{p\left(\mathcal{D} \mid H_{1}\right)}{p\left(\mathcal{D} \mid H_{0}\right)} .
$$

This log-likelihood ratio is called the Bayes factor (Kass and Raftery, 1995), which represents the strength of evidence of one hypothesis against the other. In Kass and Raftery, 1995 a scale is proposed, which we will use to interpret the size of the Bayes factor. Now, if BF is larger than 0 then the optimal hypothesis is $H_{1}$ and connection $e_{j i}$ is present in the group. If $\mathrm{BF}$ is smaller than 0 than the alternative hypothesis $H_{0}$ is more likely and therefore the connection is absent in the group.

Finally, from the likelihood of an hypothesis, we can also calculate the posterior probability of this hypothesis, for example for $H_{1}$ :

$$
p\left(H_{1} \mid \mathcal{D}\right)=\frac{p\left(\mathcal{D} \mid H_{1}\right) p\left(H_{1}\right)}{p(\mathcal{D})},
$$


where, because there are only two hypotheses and we choose $p\left(H_{1}\right)=p\left(H_{0}\right)=0.5$,

$$
p(\mathcal{D})=p\left(\mathcal{D} \mid H_{1}\right) p\left(H_{1}\right)+p\left(\mathcal{D} \mid H_{0}\right) p\left(H_{0}\right) .
$$

The posterior probability does not influence the choice of optimal hypothesis chosen using the Bayes factor, but does provide a clearer and more easily interpretable measurement of the strength of evidence in favour of the hypothesis. The larger the posterior probability is, the stronger the evidence that the optimal hypothesis is true given the measurements.

\subsubsection{Validation of ICA time series AR models}

Both Granger-causality analysis and the Bayesian topology identification rely on the assumption that the data is approximately generated by an AR model as defined in (7.4). If the dynamics of the ICA time series cannot be modeled sufficiently, the two methods becomes less reliable for the inference of brain network connectivity. To assess the goodness of the model fit, we estimate an AR model using all 20 ICA time series from one fMRI scan for each of the 64 scans in the study. The model parameters are calculated using ordinary least squares. Then, we perform a whiteness (auto-correlation) test of the residuals of each VAR model (Ljung, 1999), to test whether the AR models can sufficiently model the dynamics in the ICA time series. For model order $m=3,96.5 \%$ of the AR model residuals are white, which increases to $99.4 \%$ for $m=5$. Therefore, the results of the tests are satisfactory.

\subsubsection{Inference of the existence of a Mozart effect}

In our search for a Mozart effect, we will first choose connections with potential effects using the Bayesian selection frequency. Then we will apply the extended Bayesian method and the Grangercausality analysis to the ICA time series of the chosen connections. Based on other studies of the Mozart effect (Bodner et al., 2012; Rauscher, Shaw, and Ky, 1993; Verrusio et al., 2015; Yang et al., 2018), we hypothesise that there will be changes in the effective connectivity from and to brain networks involved in cognitive processing. Furthermore, because the subjects are listening to music we expect to find changes in connectivity between brain networks involved in auditory processing and possibly motor regions.

In the Mozart effect study, the scans in week 1 and 2 are used to infer the natural variability in brain network connectivity of the subjects. If a connection has a low variability in effective connectivity in these weeks, it is more likely that any changes in connectivity of subjects between week 2 and week 3 is due to listening to Mozart music. Finally, for the connections of which the connectivity changed between week 2 and 3, we can compare their connectivity of week 3 and 4 to see if the effect lasts even into week 4 or if it was only of short duration.

Now, given the criteria described above, we define for each method when a change in effective connectivity took place as a result of listening to Mozart music. For the Bayesian hypothesis test we define the optimal hypotheses for each week such that the collected hypotheses over all four weeks indicate a change in effective connectivity caused by Mozart music. For week 1 and 2 the optimal hypotheses should be the same for both weeks. Then in week 3, the optimal hypothesis should be the opposite of week 1 and 2 . Then, the optimal hypothesis in week 4 indicates whether the change between week 2 and 3 lasted into week 4 or not. Finally, in the Granger-causality analysis a significant difference in Granger-values of subjects should only be found between week 2 and 3, and possibly between week 3 and 4, depending on if the change lasted into week 4 or not. 

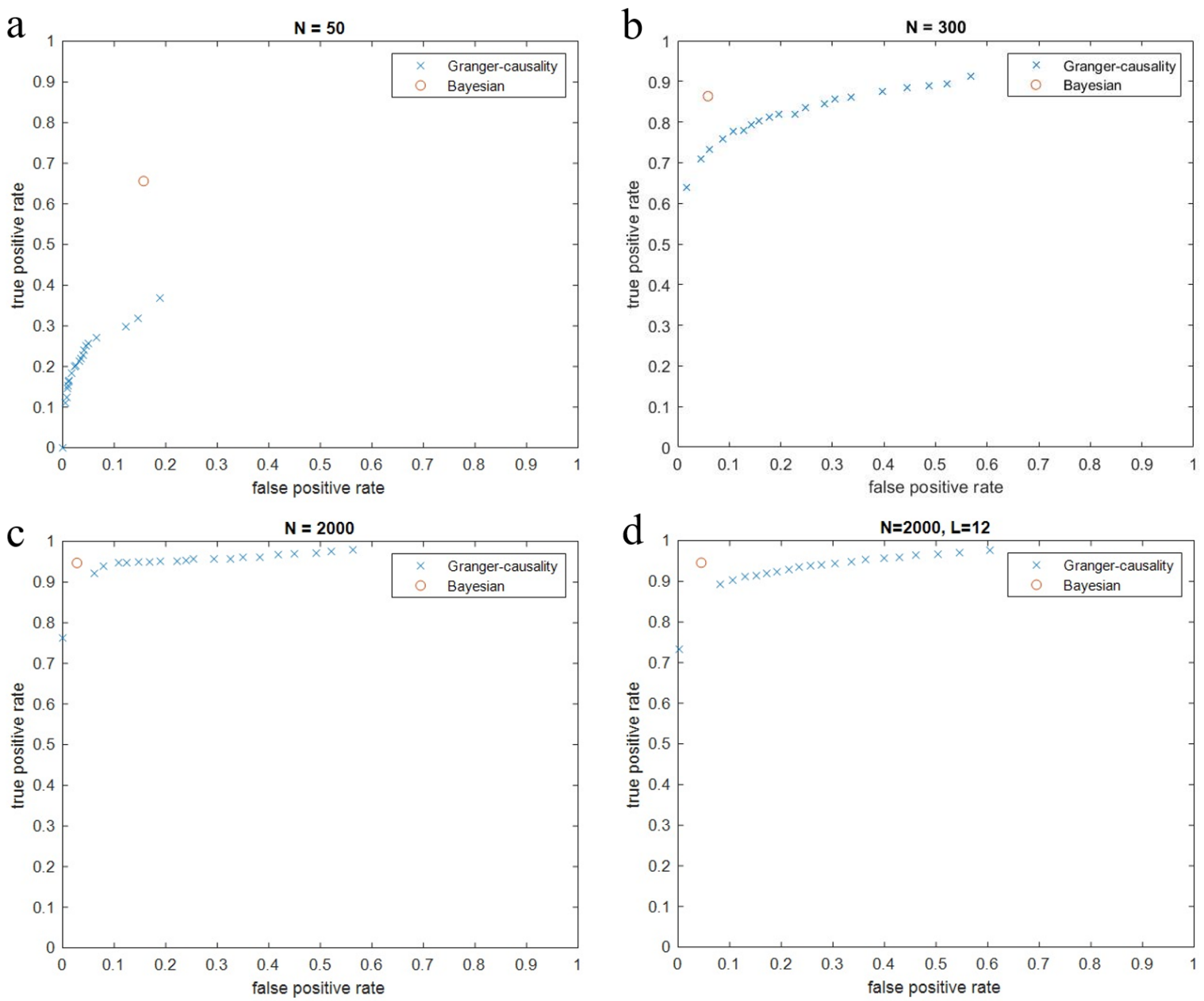

FIGURE 7.4: FPR vs TPR of the Bayesian method and Granger-causality analysis graph estimates. One blue cross indicates the TPR and FPR of the Granger-causality analysis for a given p-value $(\{0.005,0.05, \ldots, 0.95\})$. As the p-value increases the TPR and FPR of the Granger-causality analysis increases. The TPR and FPR of the Bayesian topology identification are indicated with a red circle. This is a point estimate as the Bayesian method does not rely on a threshold to determine the existence of connections. the simulation parameters (sample size $N$ and number of nodes $L$ ) are: (a) $N=50, L=6$; (b) $N=300, L=6$; (c) $N=2000, L=6$; (d) $N=2000, L=12$.

\section{Inference based on listening duration}

We will perform one more analysis of the data, by dividing the subjects' weekly scans into two subgroups based on their listening duration of Mozart music between week 2 and 3 . We rank the subjects based on listening time and choose the 8 longest listeners as the first subgroup and the remaining 8 subjects form the second subgroup. The first subgroup of longer listeners listened on average 27:14 \pm 7:07 hours to Mozart music and the second subgroup 16:19 $\pm 1: 31$ hours on average. We perform both the Bayesian hypothesis test and the paired t-test of the Granger-values for both subgroups to assess if effects are influenced by the listening duration of the subjects. 


\subsection{Results}

In this section, we will first evaluate the performance of the Bayesian topology identification against Granger-causality analysis on simulation data. Then we apply both methods to the Mozart music ICA time series to infer changes in connectivity caused by listening to Mozart music. Finally, we apply our methods on two subgroups of 8 subjects, divided based on listening duration, to assess if listening duration affects the possible detection of effects.

\subsubsection{Evaluation of methods in simulation}

The optimal performance in Figures 7.4.a-d is where TPR $=1$ and FPR $=0$, because it implies $\hat{\mathcal{G}}=\mathcal{G}_{0}$. The closer we are to this point the better the performance of the method. In Figures 7.4.a-c we see that the Bayesian method shows improvement as the data length increases. We also see that the Granger method performance improves as the data length increases, but the optimal significance threshold differs between the figures. Finally, it is clearly visible that in all of Figures 7.4.a-d the Bayesian performance is always closer to the optimal point than the performance of the Granger method for any threshold.

From Figures 7.4.a-c we notice that the Bayesian method outperforms the Granger-causality analysis for all three data lengths over all different thresholds. When comparing Figures 7.4.c and 7.4.d we see that there is barely any change in performance for the Bayesian method when the number of nodes in the dynamic network is increased as the TPR is the same in both figures and the FPR is only slightly larger for the twelve-node networks. However, for the Granger-causality analysis, the TPR of all thresholds decreases. Furthermore, we also notice a small increase in the FPR of the Granger-causality analysis, which becomes increasingly large for low thresholds. Lastly, one more important detail is that when we inspect Figures 7.4.a-c for the Granger-causality analysis, the optimal performance for each of the figures does not belong to the same threshold, instead it depends on the data length $N$. But based on the change in performance for each threshold as $N$ becomes large, it appears the $5 \%$ threshold will be the optimal threshold for large $N$.

While the Bayesian method outperforms the Granger-causality analysis in our performance evaluation, there are other advantages and disadvantages to consider. First of all, the Granger-causality analysis is a relatively simple method and the computational speed is fast even for a large number of nodes $L$ in the network, while the Bayesian method does not scale well in terms of computational complexity as the number of nodes increases. Furthermore, a disadvantage of the Bayesian method is the reliance on good initial conditions of the EM algorithm to determine the hyperparameters of the parameter prior.

\subsubsection{Inference of the Mozart effect}

From the initial search for the Mozart effect using the Bayesian selection frequency of the connections over the four weeks, we have chosen six connections, in which the selection frequency indicate that there might be a change in the optimal hypothesis between week 2 and 3, i.e. before and after the subjects listened to Mozart music. The Bayesian selection frequencies of these connections are reported in Figure 7.5.

In Figures 7.5.a and 7.5.b we found two connections inbound to the fronto-parietal right network and in 7.5.f we see the connection from the anterior default mode network to the dorsal attention network. These connections are of interest, because the default mode networks and the frontoparietal right network are involved in cognitive processing (Marek and Dosenbach, 2018; Raichle, 

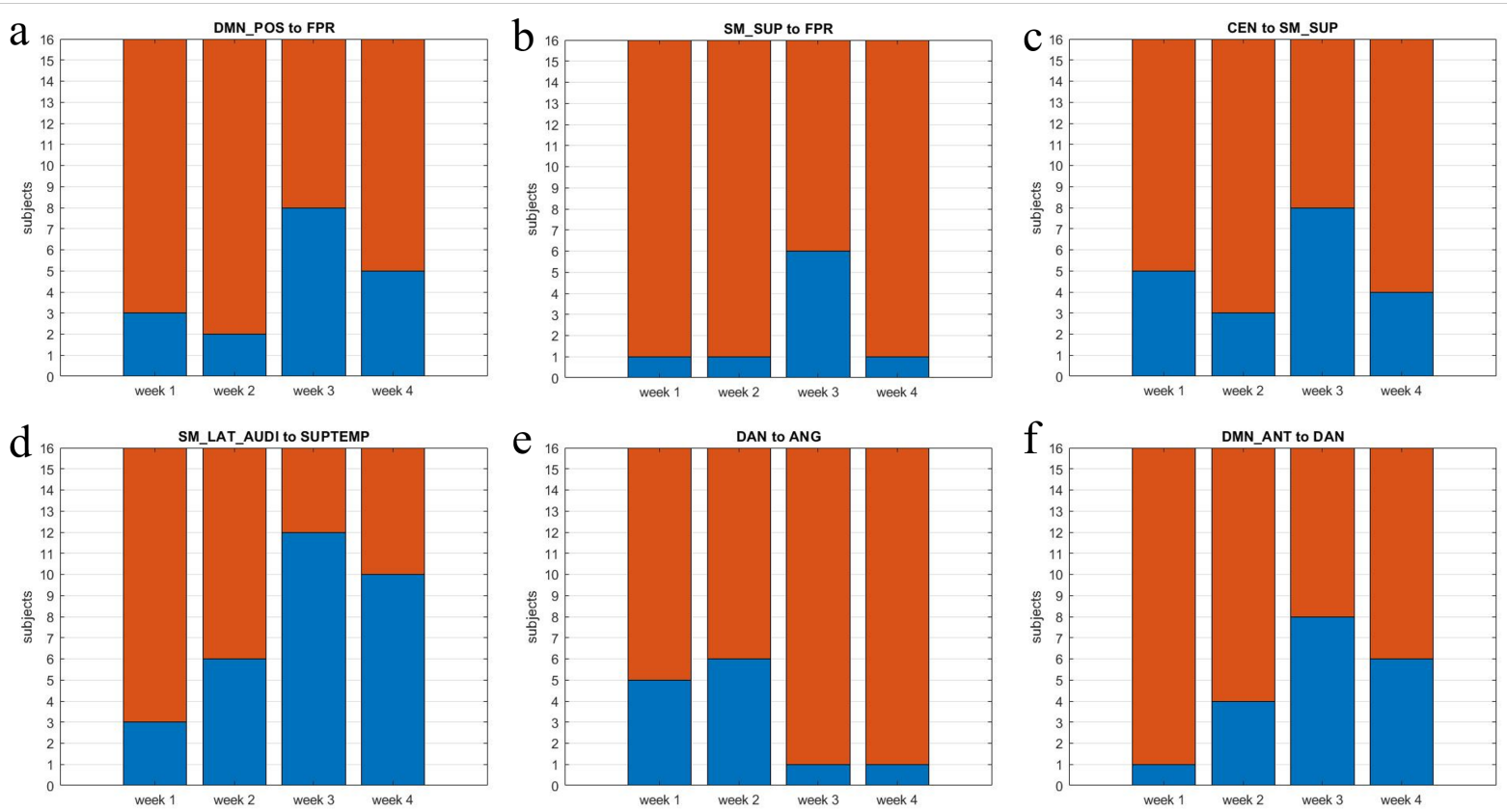

FIGURE 7.5: Selection frequencies of four connections of with possible effects of interest. Here, red indicates the number of subjects that did not have the connection in that week and blue indicates the number of subjects that did have the connection. Connections are: (a) from the posterior default mode network to the fronto-parietal right network; (b) from the sensori-motor superior network to the fronto-parietal right network; (c) from the central executive network to the sensori-motor superior network; (d) from the lateral sensori-motor network to the superior temporal gyrus; (e) from the dorsal attention network to the angular gyrus; (f) from the anterior default mode network to the dorsal attention network.

2015). In Figure 7.5.c we see the selection frequency of a connection involving the central executive network, which is involved in cognitive processing (Miller and Cohen, 2001). Then, in Figure 7.5.d we show the selection frequency of the connection from the sensori-motor, lateral and the superior temporal gyrus. Figure 7.5.d is of special interest because first of all it has the largest selection frequency for any connection over all scans, with 12 out of 16 subjects showing positive evidence of the connection. Furthermore, it has a relatively small drop in selection frequency in week 4 relative to week 3 . Both of the brain networks involved in the connection are involved in auditory processing in the brain (Chen, Penhue, and Zatorre, 2008; Koelsch et al., 2005). Finally, in Figure 7.5.e we show a connection from the dorsal attention network to the angular gyrus. This connection is of interest, because a low variability large selection frequency was detected in week 1 and 2, which almost completely disappears in week 3 and does not increase at all in week 4 . This could indicate that effective connectivity in connections can decrease as a result of listening to Mozart music. In summary, we have found a small selection of potential effects in our analysis of the selection frequency. However, while we see some changes in selection frequency between week 2 and 3, the effects are far from universal across all subjects.

Next, we assessed the optimal group hypothesis of the 16 subjects for each week for the six connection we reported in Figure 7.5. We have presented the results in Table 7.2. In Table 7.2 we report the optimal hypothesis of each connection for each week, its Bayes Factor BF and its posterior probability $p\left(H_{\text {opt }} \mid \mathcal{D}\right)$. Now, from Table 7.2 we notice the connection from the posterior default mode network to the fronto-parietal right network and the connection from the central executive network to the sensori-motor superior network. For these two connections the optimal hypothesis in week 1 and 2 was $H_{0}$, and the optimal hypothesis in week 3 was $H_{1}$, indicating a change in effective connectivity of these connections after the subjects listened to Mozart music. 
TABLE 7.2: Results of the extended Bayesian method on the ICA time series in the Mozart effect study.

\begin{tabular}{|l||c|c|c|c|c|c|c|c|c|c|c|c|c|}
\hline \multirow{2}{*}{ Connections } & \multicolumn{3}{|c|}{ week 1 } & \multicolumn{3}{c|}{ week 2 } & \multicolumn{3}{c|}{ week 3 } & \multicolumn{3}{c|}{ week 4} \\
\cline { 2 - 15 } & $H_{\text {opt }}$ & BF & pos & $H_{\text {opt }}$ & BF & pos & $H_{\text {opt }}$ & BF & pos & $H_{\text {opt }}$ & BF & pos \\
\hline DMN_ANT to DAN & $H_{0}$ & -40.08 & 1 & $H_{0}$ & -33.62 & 1 & $H_{0}$ & -7.305 & 0.97 & $H_{0}$ & -29.65 & 1 \\
\hline DMN_POS to FPR & $H_{0}$ & -41.26 & 1 & $H_{0}$ & -44.80 & 1 & $H_{1}$ & 1.890 & 0.72 & $H_{0}$ & -45.07 & 1 \\
\hline CEN to SM_SUP & $H_{0}$ & -38.88 & 1 & $H_{0}$ & -47.75 & 1 & $H_{1}$ & 8.266 & 0.98 & $H_{0}$ & -27.03 & 1 \\
\hline SM_LAT to SUPTEMP & $H_{0}$ & -20.78 & 1 & $H_{1}$ & 37.46 & 1 & $H_{1}$ & 237.7 & 1 & $H_{1}$ & 72.34 & 1 \\
\hline DAN to ANG & $H_{0}$ & -24.73 & 1 & $H_{0}$ & -39.28 & 1 & $H_{0}$ & -109.5 & 1 & $H_{0}$ & -74.87 & 1 \\
\hline SM_SUP to FPR & $H_{0}$ & -53.55 & 1 & $H_{0}$ & -70.83 & 1 & $H_{0}$ & -53.21 & 1 & $H_{0}$ & -86.71 & 1 \\
\hline
\end{tabular}

The results are summarised for each of the four weeks, where we indicate for each week the optimal hypothesis, the Bayes factor (BF) and the posterior probability (pos) of the optimal hypothesis in that week.

TABLE 7.3: Results of the inference based on the listening duration of subjects from the Bayesian method.

\begin{tabular}{|l||c|c|c||c|c|c|c|c|c||c|c|c|}
\hline \multicolumn{1}{|l|}{ Connections } & \multicolumn{3}{c||}{ week 1 } & \multicolumn{3}{c||}{ week 2 } & \multicolumn{3}{c|}{ week 3 } & \multicolumn{3}{c|}{ week 4 } \\
\cline { 2 - 14 } & $H_{\text {opt }}$ & BF & pos & $H_{\text {opt }}$ & BF & pos & $H_{\text {opt }}$ & BF & pos & $H_{\text {opt }}$ & BF & pos \\
\hline DMN_ANT to DAN & $H_{0}$ & -15.09 & 1 & $H_{0}$ & -25.46 & 1 & $H_{1}$ & 2.43 & 0.77 & $H_{0}$ & -28.49 & 1 \\
\hline DMN_POS to FPR & $H_{0}$ & -21.37 & 1 & $H_{0}$ & -56.62 & 1 & $H_{1}$ & 13.77 & 0.99 & $H_{0}$ & -10.76 & 1 \\
\hline
\end{tabular}

The results are summarised for each of the four weeks, where we indicate for each week the optimal hypothesis, the Bayes factor (BF) and the posterior probability (pos) of the optimal hypothesis in that week. Only two connections are shown here, which both only showed a change in optimal hypothesis only for the subgroup of longer listening subjects. The evidence and optimal hypothesis of the shorter listening subgroup is not shown as they contain no effects of interest.

Furthermore, in both connections the change did not last into week 4, as the optimal hypothesis was once again $H_{0}$. Next, we note in Table 7.2 that the connection from the sensori motor lateral network to the superior temporal gyrus shows strong evidence in favour of $H_{1}$ in week 3 , however, the optimal hypothesis in week 1 and 2 changes from $H_{0}$ to $H_{1}$ and therefore we conclude that the natural variability of this connection before listening to Mozart music is too large to detect a change in effective connectivity caused by the Mozart music. Finally, we note that for the other three connections, the optimal hypothesis for both week 1 and 2 did not change, but there was also no change in optimal hypothesis between week 2 to week 3 and as such there was no significant change in the effective connectivity of the connections in the group of all 16 subjects. The brain networks involved in the connections for which a change in optimal hypothesis occurred only between week 2 and 3, have been summarised in Figure 7.6.

Finally, for all six connections in Figure 7.5, we found no significant difference in Granger-values between week 1 and 2. Then we compared the Granger-values of week 2 and 3 and found significance of the paired t-test only for the connection from the anterior default mode network to the dorsal attention network with $p=0.035$. Next, we inferred only for this connection if there are any differences in Granger-values between week 3 and 4 and found no significant difference between the two weeks, indicating that the effective connectivity change persisted into week 4 .

\subsubsection{Inference based on listening duration}

In our final test, the subjects are divided into two groups of 8 subjects. The subjects in group 1 listened on average 27:14 7:07 hours to Mozart music between week 2 and 3 and the subjects in group 2 listened on average 16:19 \pm 1:31 hours to Mozart music. Both methods were repeated for the new subgroups.

Now, using the Bayesian hypothesis test on both subgroups, we have found two connections that showed an effect only for the subgroup with a longer listening duration, but no effect for the group with a shorter listening duration. These two connections are presented in Table 7.3. We only show the BF and optimal hypotheses for the subgroup which listened for a longer duration, 
DMN_POS to FPR

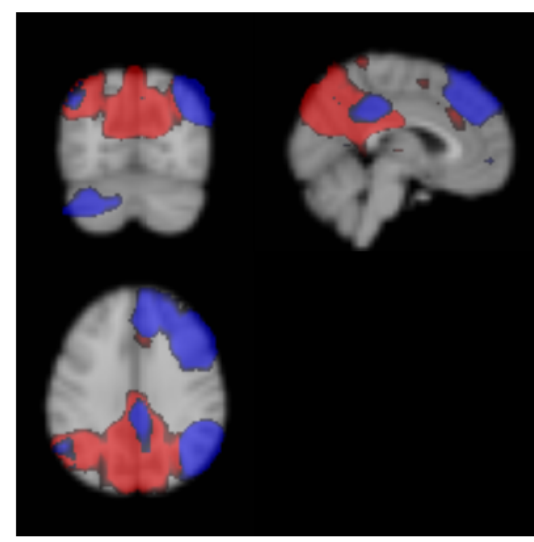

DMN_ANT to DAN

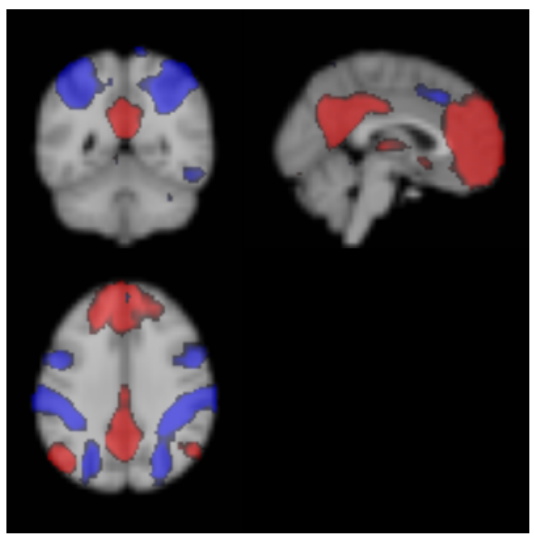

CEN to SM_SUP

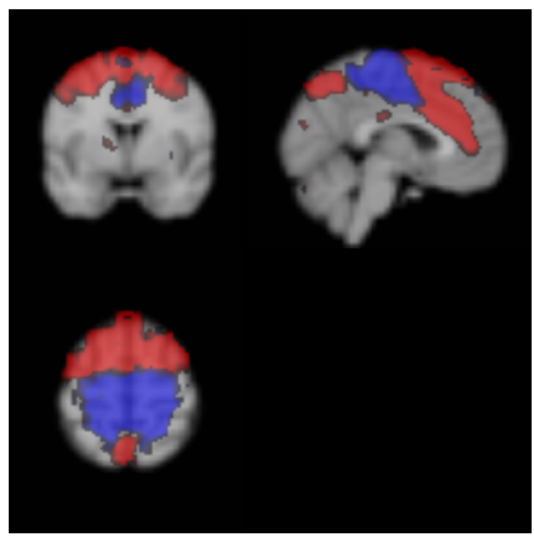

FIGURE 7.6: Brain networks in which the connections were found to have the most important changes in effective connectivity after subjects (full group or long listening subgroup) listened to Mozart music. Here the direction of the connection is from the red network to the blue network.

as no change in optimal hypothesis was found between week 2 and 3 for the shorter listening subgroup. The other connections which were selected in Figure 7.5, were not favoured by one of the subgroups over the other and are therefore also not presented here.

The first connection in Table 7.3, from the anterior default mode network to the dorsal attention network, which showed no change in optimal hypothesis between week 2 and 3 for the group of 16 subjects, shows positive evidence in favour of $H_{1}$ in week 3 for the subgroup of longer listeners. As such there is a change in optimal hypothesis between week 2 and 3 for the longer listening subgroup. The change in optimal hypothesis between week 2 and 3 did not last in week 4 , as such this effect was of short duration. The second connection in Table 7.3, from the posterior default mode network to the fronto-parietal right showed weak evidence in favour of $H_{1}$ in week 3 for the group of 16 subjects and as such there was a change in optimal hypothesis between week 2 and 3. This connection shows strong evidence in favour of $H_{1}$ in week 3 for the subgroup of longer listeners, as such the change in optimal hypothesis between week 2 and 3 in the full group of 16 subjects was predominantly caused by the longer listening subjects. Similarly to the inference in the full group of 16 subjects, the change in optimal hypothesis between week 2 and 3 did not last into week 4, thus the effect was of short duration. A visualisation of the networks involved in these connections can be found in Figure 7.6.

Finally, no results were found for the significance of Granger-values for both groups. All comparisons between weeks for both long and short listeners had no significant change in effective connectivity between any of the weeks for all selected connections in Figure 7.5.

\subsection{Discussion}

In this study, we illustrated, using simulation data, that the Bayesian topology identification outperforms the Granger-causality analysis, especially when not much data is available. The Bayesian method was extended to enable it to test hypotheses on the existence of a connection for each week separately. The optimal hypotheses of each week are combined to draw conclusions on changes in the effective connectivity over the weeks in the Mozart study. Then we applied the extended Bayesian method and the Granger-causality analysis to the Mozart effect data and found a number of connections with changes in effective connectivity after the subject listened to Mozart music. Finally, we split the subjects into two groups based on listening duration and found that for two 
connections a change in effective connectivity could only be detected in the subgroup of longer listening subjects.

We evaluated the Bayesian topology identification as an alternative to the popular Grangercausality analysis for the inference of brain network connectivity. The methods are compared through their performance in estimating a graph of the effective connectivity using simulated data with a known ground truth connectivity. Herein, we expected that the Bayesian method should have an advantage in the inference over the Granger-causality analysis through the use of a parameter prior and by modelling the AR model parameters as random variables. The performance measurements in Figure 7.4 show that the Bayesian method performs better than the Granger-causality analysis for short data lengths using the simulation data. As the data length increased we saw that the Granger-causality analysis performance more closely matched that of the Bayesian method. Thus, for fMRI data studies, where often not many data points are available due to the slow sampling rate of fMRI measurements, it seems that the Bayesian method has a distinct advantage in the inference of brain network connectivity over Granger-causality analysis.

In the study of the Mozart effect, we applied both the Bayesian method and the Granger-causality analysis to the ICA time series of the subjects. Here, we focused on the Bayesian method, because we observed it performed better than the Granger-causality analysis in the evaluations of performance using simulated data. First, using the Bayesian hypothesis test we detected a change in effective connectivity of multiple connections after subjects listened to Mozart music. For the full group of 16 subjects, we detected a change in optimal hypothesis for the connection from the central executive network to the dorsal attention network, which was of short duration. The central executive network is involved in maintaining and manipulating working memory and performs goal-oriented decision making (Miller and Cohen, 2001). Therefore the change in effective connectivity could be an indication of a change in cognition.

Next, the connection from the posterior default mode network to the fronto-parietal right network also showed an increase in effective connectivity after listening to music as measured by the Bayesian method, although the evidence that $H_{1}$ was the optimal hypothesis in week 3 was only weak. When we split the subjects into subgroups based on listening duration, it becomes apparent that the effect predominantly occurs in the longer listening subjects and was of short duration. The default mode network is involved in emotional processing, self-referential mental activity, and the posterior default mode network in particular involves recollection of past experiences (Raichle, 2015). The fronto-parietal right network is involved in cognitive control (Marek and Dosenbach, 2018). This change in effective connectivity between two networks involved in cognitive processing is an interesting result as it could indicate the existence of the Mozart effect.

Finally, the connection from the anterior default mode network to the dorsal attention network was detected to have a significant change in effective connectivity between week 2 and 3 by the Granger-causality analysis for the whole group of subjects. In the Bayesian method a change in optimal hypothesis was detected, but only for the subgroup of longer listening subjects. However, the two methods disagree on whether the effect was of short duration or not and as such we cannot draw any strong conclusions on the duration of the change. Overall, the evidence that this connection was present in week 3 from the Bayesian method was not strong and the connection was only present in the subgroup. However, it is supported by the Granger-causality analysis results, and as such we are more confident in the result that an actual change in effective connectivity happened after listening to Mozart music. We have mentioned the functionality of the default mode network already above. Furthermore, the dorsal attention network is involved in the orientation of attention to external stimuli (Vossel, Geng, and Fink, 2014). Thus the brain networks in this connection are involved in cognitive processing. The change in effective connectivity caused by Mozart music, detected by both methods, could indicate evidence of the Mozart effect. 
Compared to the Granger-values, which are a measurement of connectivity strength and for which statistical tests exist to infer a difference in connectivity between groups of subjects, the extended Bayesian method can only test binary hypotheses of the effective connectivity. In future development of the Bayesian method, one of our goals will be to extend the Bayesian hypothesis test to provide a quantitative measure of the connectivity strength. This could help us draw conclusion on effective connectivity change in the connection from the sensori-motor lateral network to the superior temporal gyrus, where even though the optimal hypothesis was $H_{1}$ in both week 2 and 3 , we see a very large increase in the evidence BF in week 3 as compared to week 2 , but with our currently available binary hypotheses on effective connectivity, we cannot confirm if this indicates a change or not.

Although we had originally mandated a minimum of 2 hours a day of listening to music, this minimum listening duration appeared to be insufficient to cause changes in effective connectivity in all subjects for the connections involving the anterior and posterior default mode networks. The listening duration in other studies of the effect of Mozart music on epileptic seizure frequency was at least 2 hours a day for 15 days (Coppola et al., 2015). In another study (Bodner et al., 2012) patients were exposed to Mozart music for a year. Therefore, in future work we would like to increase the minimum listening duration of subjects, to verify whether in that case effects will be more universal across subjects. Furthermore, because listening to Mozart music for more than 2 hours a day might be hard to accommodate for subjects, it might be necessary to increase the time interval between scan 2 and 3 to reduce the daily listening time to a manageable duration.

This was an exploratory study of the Mozart effect by inferring changes in brain network connectivity using ICA time series from fMRI data. As far as we are aware, this study is the first of its kind in measuring effective connectivity changes caused by Mozart's Sonata K448. Furthermore, it is the first time that the Bayesian topology identification algorithm is applied to fMRI data. We have found changes in cognitive processing in subjects, some of which were predominantly in the subjects with a longer listening duration. More effects were hinted at by the Bayesian selection frequencies in Figure 7.5, but could ultimately not be detected through the Bayesian hypothesis test. We are hopeful that in future studies, with increased listening duration of subjects, more changes in effective connectivity caused by Mozart music will be found. 

Chapter 8

\section{Discussion and conclusions}



Clearly medicine is at the age in which words such as precision medicine, multi-modal approach, computer-aided diagnosis, machine learning, and non-invasive treatments are commonly used. In this context, neuroscience needs more translational research-from mathematical models to empirical behaviours - and innovation in order to have modern tools for assessing brain disorders in a non-invasive, personalised and automated way by bridging modeling techniques with real life applications. With neuroimaging techniques, such as resting-state fMRI, brain functional connectivity is usually represented with correlation matrices-static functional connectivity. These matrices can then be displayed as coloured functional maps overlaid on a structural brain image. However, these mappings of brain networks activation are static representations of brain functioning, and are mainly used in case of brain surgery (or for research). Indeed, static activation maps of brain functions do not provide robust insight in neuropsychiatric disorders yet. Since mental disorders are only diagnosed through psychological tests and interviews, there is a clear need for having also objective schematic or map representation of the brain dynamic connectivity (not static) that are involved in mental disorders in order to help and confirm a diagnosis. Having a clear picture/video of what is going on in neuropsychiatric patients' brains can also help developing tools for treatment such as brain stimulation techniques. So far, this is not used in clinics, and scanner vendor software does not enable visualizations of such neurodynamics.

Finding new ways of modeling reliably brain dynamic connectivity that distinguish neuropsychiatric patients from healthy, i.e. robust biomarkers of the diseases, could greatly help the new research field of psychoradiology. For the past decade, several methods have been investigated that have mainly applied a sliding-window version of static connectivity measurements (correlation matrices) in healthy subjects. We have presented in this thesis new ways of extracting dynamics metric of the brain, namely the Granger causality, the time of coherence, and the emulative power between large-scale networks. We have shown their potential in helping clinician understanding the mechanisms of action of these brain disorders. In this section, the main findings of the novel methods for measuring brain dynamics when applied on adolescents with autism spectrum disorder (ASD) and elderly patients with accelerated cognitive ageing (ACA) are summarised and discussed. The limitations of the studies presented in this thesis and the future work needed for applying these techniques in clinical practise, are also described in this section.

\subsection{Comparing causality, coherence, and evolution dynamics}

For decades correlation matrices have been created and compared in order to find abnormal network connectivity in neuropsychiatric disorders. These connectivity matrices inform us only on the strength and the spatial distribution of the defective networks, which in turn indicate segregation/integration issues of nodes within brain networks. However, many psychiatric disorders display normal functional use and spread of the resting-state networks. For these diseases, anapproach for measuring neurodynamics is needed, especially since the variance in performance of cognitive tasks is better explained by a dynamic analysis rather than a static one (Liégeois et al., 2019).

In that respect, we have investigated here new ways of modeling dynamics in the brain using resting-state fMRI. We first have evaluated the effective connectivity in terms of Granger causality (GC) between resting-state networks. It has shown that, in autism, the causality between the ventral attention network (VAN), the salience-executive network (SEN) is challenged. However, these findings were relevant only in a second resting state session (after an emotion processing task-fMRI session). Hence, the causality between the VAN and the SEN are only weakened in autism after challenging (priming) the brain of the adolescents with autism. These findings were 
in line with current research in imaging autism spectrum disorder. Indeed, the VAN and SEN networks are known to be utilised during working memory tasks and emotional processing, which is often deficient in autism. In accelerated cognitive ageing (ACA) patients, the effective connectivity between large-scale cognitive networks, such as the default mode, dorsal attention, and salience networks has also shown stronger causality as compared to age-matched healthy subject. This difference is present when comparing young adult to elderly as well. Hence, our theory of an accelerated cognitive ageing holds. Nevertheless, like in autism, the causality changes were depending on the resting-state sessions. Therefore, Granger causality has not been a strong and reliable biomarker of ACA-we obtain around 67\% accuracy in our classifier trained on GC measurements. Due to the lack of robustness, we have investigated a new method that attempts to describe the true effective connectivity topology of the brain. Indeed, in Chapter 7, a new Bayesian method for inferring effective connectivity between networks has been introduced. This Bayesian search appears to be more powerful at detecting changes in dynamic connectivity in simulated fMRI time series than the GC. This new Bayesian approach is also useful for detecting changes in dynamic connectivity of cognitive and perceptive networks in real fMRI data of healthy participants subjected to Mozart music-the effective connectivity (with Bayesian search approach) between cognitive networks (default mode network; central executive network) and attention, and sensorimotor networks (dorsal attention networks and superior motor cortex) has shown changes in strength after participants listened to Mozart music for a week (in average 2 hours per day). This might be relevant for further research in music therapy and brain cognitive enhancement.

As a second dynamics analysis method, the coherence in terms of phase synchronicity between resting-state networks, has been assessed. This has been performed by the wavelet coherence (WCoh) approach. In case of autism, the WCoh-derived 'in-phase time of coherence' between the ventral attention networks and the lateralised executive-control networks (fronto-parietal left and right) are lower: the two pairs of networks were notably synchronised for shorter duration as compared to controls (when assessed at around $10 \mathrm{~s}$ wavelength activity periods). This time of in-phase coherence has allowed us to distinguish ASD from healthy controls at $86.7 \%$ accuracy with SVM-based classifiers. In ACA, similar results of abnormality in the in-phase time of coherence have been found mainly between the default mode network, the salience networks and two visual networks (medial and occipital) - this has resulted in $77.1 \%$ accuracy in diagnosis of ACA using the decision-tree classification algorithm trained with these coherence-based features. As opposed to autism, for the ACA patients, the duration of synchronicity between the networks was higher, which is corroborated by recent research on a posterior-to-anterior shift in activity linked to cognitive ageing - the PASA model.

Finally, we have investigated a promising new dynamics metric that does not rely on effective connectivity, nor on time-frequency (coherence) analysis, but on the evolutionary game theory. Indeed, we have applied a simple model that describes how the demographic rate of agents/players in populations/species evolves in time, when agents replicate strategies of the other populations' players. In the brain dynamics context, populations are resting-state networks. When we derived, from the EGN model, our novel metrics of in-, out-, and net-emulative powers (capacity to emulate activity) of known resting-state networks, we have demonstrated that this new dynamics measurement can be relevant and insightful in case of ACA: we have found significant differences in the emulative powers of the dorsal attention network (DAN) and the default mode network (DMN). The emulative powers of the DAN and DMN have actually allowed us to distinguish ACA patients from controls at $82.9 \%$ accuracy, with a linear discriminant analysis classification. Again, these networks have been proven to be greatly challenged in the ageing process, and link to fluid intelligence decline.

Overall, in ACA patients, the 3 above mentioned dynamics analysis methods can be ranked-in their relevance as reliable biomarkers of brain diseases-as EGN > WCoh > GC. However, the 
EGN method did not perform as good for autism (results not shown in this thesis). Therefore we can assume that the preferred dynamics methods are disease-dependent. In any case, as corroborated by Liégeois et al., 2019, we obtain the best performances in classifying an ACA by combining the 3 methods (EGN, WCoh, and GC) with the static functional connectivity (sFC)-peak at 95.8\% accuracy, with an SVM classifier. Therefore, perhaps we should not seek the best method to describe neuropsychiatric disorders, but rather search for the best metric combination.

Regarding the classification techniques applied in this thesis (Chapter 5 and 6), it is worth noting that advance neural networks and other unsupervised algorithms that can learn fMRI-based disease features themselves arealso possible to implement. However, our applied supervised algorithms are adequate when features have been a priori extracted, and they do not need a high amount of data to show significant performances in binary classification (healthy/non-healthy).

\subsection{Limitations}

\subsubsection{Preprocessing, parcellation and global regression for replicability}

One of the main limitations in this thesis is that each study is performed following one way of preprocessing the rs-fMRI data. Using FMRIB Software Library (FSL), we have performed the different preprocessing steps for cleaning and normalizing rs-fMRI data: co-registration to MNI space; spatial and temporal filtering; and no global regression of mean signals. The global regression was not carried out, since its utility in improving SNR and the subsequent statistical power is still debated (Li et al., 2019; Murphy and Fox, 2017; Xu et al., 2018). In addition, it has been shown that global regression can have negative effect on group difference results in case of fMRI study of autism (Gotts et al., 2013). Also, when using the ICA, global signal regression can lower the sensitivity of the decomposition (see FSL - MELODIC ${ }^{1}$ documentation), and we can simply compensate the regression by discarding the noisy networks, since the ICA method can separate noise (global signal) from activity-related network time series. Even though we did not test the effect of changing the preprocessing steps throughout the research, our methodology is consistent, and the findings from the dynamics analyses can be compared.

We had, nevertheless, one major difference in our data acquisitions. More specifically, ACA and Mozart studies were performed using a multi-echo fMRI acquisition, whereas for the study of autism single echo data were used. This difference in rs-fMRI acquisition could lead to difference in estimation of dynamic pattern in the brain of the participants. Though, we expect that the multi-echo acquisition is a better choice as compared to single-echo fMRI, since it has been proven to be one of the best ways of cleaning the rs-fMRI data (Dipasquale et al., 2017). It also allows to increase the SNR in BOLD signal, and subsequently the power of the network analysis (Lombardo et al., 2016). Still a thorough analysis should be carried out to shed light on the added value of such a multi-echo data cleaning for detecting abnormal dynamics in neuropsychiatric disorders.

With the fMRI acquisition and preprocessing limitations, research in the field of psychoradiology should focus on reliability of the methods to extract relevant biomarkers for neuropsychiatric diseases. Indeed, to be used in clinics, biomarkers of mental disorders should be replicable. In $\mathrm{fMRI}$, the main issue is that to assess simple static connectivity between large-scale resting state networks, a thousand pipeline configurations are already possible (from acquisition methods to the dynamics analysis technique). Indeed, if we take only a choice of two possible techniques at each of ten usually executed steps of acquisition and preprocessing (TR of the fMRI; number of scans; number of echoes; network extraction method; head-movement correction; fMRI cleaning

\footnotetext{
${ }^{1}$ https://fsl.fmrib.ox.ac.uk/fsl/fslwiki/MELODIC
} 
method; regression analysis; slice-timing correction; temporal filtering; and spatial filtering), we have $2^{10}$ possible configurations for the analysis. By taking a more reasonable average number of five choices per step, we get $5^{10}$, i.e. around 10 million, possible pipeline configurations to extract biomarkers. So, clearly a consensus on how data are collected and processed would make fMRIbased biomarker extraction more reproducible. More details are presented in the future work discussion.

The choice of brain networks or ROI extraction for further analysis is also limited by the functional brain parcellation issue. Can we fully trust brain functional mapping (parcellation) based on a few participants average maps? Maybe not totally, especially, since several studies have shown the functional brain mapping into multiple areas or networks are subject-dependent (Salehi et al., 2020). Hence, instead of basing the functional networks solely on a group-ICA, a solution might be to perform individual parcellation as in (Wang et al., 2015), where the authors used an average surface-meshed atlas of (Yeo et al., 2011), and use an iterative back-projection onto individual brain. However, the main issue with this approach is that this procedure is time consuming-meshing of the brain can take several hours for one participant. The choice of the size and number of networks in the parcellation of the brain relates also directly to the choice in the number of nodes in a dynamic network models; hence, it is also a limitation in the post-processing steps. This is further discussed in the next subsection on limitations of the dynamics methods.

Overall, the limitations we have throughout the thesis are based on the choices of pre-processing step methods (i.e. data cleaning and normalization steps), and the way we parcellate the brain into functional maps to later derive group differences. In the era of precision medicine, other solutions might be possible, and certain consensus should be reached to ensure reliability in case of positive results in finding relevant brain markers of a mental disease. Nevertheless, in general, our methodology is consistent, and thus we are confident in the validity of the results. Finally, refinement of the models could benefit psychoradiology search for reliable biomarkers in other neuropsychiatric disorders such as MDD, sleep disorders, or schizophrenia. Indeed, improvement in the post-processing steps and classification pipeline can be further developed, e.g. using multi-modal approaches and disease-specific image acquisitions (more details in the future work section).

\subsubsection{Limitations in the dynamics method analyses}

As seen in the discussion parts of the different chapters of this thesis, each of our models of between-networks dynamics has their limitations. The newest and most powerful method to describe abnormalities in ACA patients is the evolutionary game theory on network (EGN). The derived emulative powers have the limitations of being assessed in a few large-scale networks. However, the original idea behind the EGN method is to enable prediction of brain dynamics, based on the emulative weight matrices, if some nodes of the global brain networks were to be missing (in case of brain injury for example; Madeo et al., 2017). In the latter paper, the authors actually advise to use more than 77 nodes to have robust estimation of the game parameter matrices, and thus reliable predictions. In Chapter 6 of this thesis, we use 14 nodes in the models (14 resting-state networks). However, we did not try to predict network activation dynamics, but we rather assessed the power of the weights/parameters of the models (the emulative powers) and groupwise compared them-where the control group acts as the ground truth. Hence, the limitation of the use of a few nodes that leads to less predictive estimators of networks connectivity can be relaxed. Another limitation in the EGN model is the assumption that networks behave and evolve as the replicator dynamic agent. Other dynamic models in evolutionary game theory are possible and might be more biologically relevant (Madeo and Mocenni, 2015; Weitz et al., 2016), and perhaps even more informative. Nevertheless, the replicator dynamics has been, in our case, 
a reliable assumption-we found significant differences in ACA emulative/replicative powers, as compared to controls.

Regarding the wavelet coherence, it has been our best method for describing autistic brain dynamics abnormality (and the second-best method in case of ACA). However, the time of coherence might not be the best coherence-map derived metric. Indeed, if static connectivity is strong between two networks, there are chances that the time of in-phase coherence in a specific range of frequency is of long duration. In addition, coherence maps (spectrograms) are good at displaying transient effect, i.e., a change in spectrum power over time. So, perhaps, a metric based on transient measures would be favorable and more informative in case of describing pathological dynamics. Also, the anti-phase time of coherence is not so prominent at being a biomarker of neuropsychiatric disorders, albeit having shown strong applicability in assessing anti-correlated dynamics between the default mode network and other brain networks (Chang and Glover, 2010) in healthy subjects. This is most probably due to the fact, that in our studies we do not regress out global signal, which can change significantly results of anti-correlated network activity.

The Granger causality is our least favorable biomarker of mental disorders or cognitive changes (Chapter 6 and 7), and its use in fMRI study is often debated. Indeed, Granger causality has been shown to describe true neural directed connectivity, even when applied on BOLD (fMRI) signals; and the GC between the ventral and dorsal attention networks is associated with enhanced or reduced behavioural performance depending on the causal direction (VAN $\rightarrow \mathrm{DAN}$, or DAN $\rightarrow \mathrm{VAN}$ ) (Wen, Rangarajan, and Ding, 2013). However, making strong claims of Granger causality pattern between groups (e.g. patient vs controls) should be done with care (Smith et al., 2012). In our case, we have performed the GC analysis between a few large-scale networks, which can hinder the true causality inside a large numbers of brain regions, since it should be preferred to assess these models with at least 50 nodes/networks to have confidence in the existence of the effective connectivity (see Chapter 7). A solution for this limitation is to perform GC not between networks, but rather within the networks. The within-network effective connectivity has indeed been shown to be relevant to describe cognitive maintenance in case of healthy ageing (Tsvetanov et al., 2016). In order to address this issue of having rather weak biomarkers using between-network GC strengths, a refinement could be to only assess pairwise connections that have strong evidence to be effectively connected through a Bayesian search-new methods of effective connectivity described in Chapter 7. This could inform the investigator on the trustable directed (effective) connection before applying the measurement of the strength of the causality using GC. This would hypothetically improve the effective connectivity modelling of brain dynamics in rs-fMRI.

Overall, perhaps, the presented methods are relevant at different levels of abstraction, and should not be tested upon the same number of nodes extracted from the same processing pipeline. Though, these dynamics methods become great descriptors of the ACA when they are combined-we obtain high classification accuracy (> 85\%) by combining EGN, WCoh and GC. However, if the dynamics are taken at different level of abstraction, using different preprocessing pipeline, we might be able to witness even stronger classification performances, which lead to better description of brain dynamics in neuropsychiatric disorders. Indeed, as mentioned before, GC might benefit from a higher number of nodes, and wavelet coherences might be more insightful with a global regression to extract anti-phase coherence, while EGN could stay with the same processing pipeline. This refinement of each dynamics model might help refining the top-down and bottomup integration problem we have when analyzing brain dynamics at the voxels or network levels, as explain in the next subsection. Finally, we have limited all our studies (Chapter 2-7) to the assessment of between-network dynamics. Hence, we have looked solely at the integrative properties of the networks. The segregation of the networks might also be of interest, and could be assessed through within-network dynamics investigations-as described earlier. 


\subsubsection{The top-down and bottom-up integration issue}

As mentioned earlier in this section, the novel dynamics techniques investigated here are most probably insightful at different levels of abstraction. These levels are located somewhere in the top-down, and bottom-up brain processing. More specifically, rs-fMRI shows meso-to-macro scale dynamics, that is, the activity of a large population of neurons. For example, the BOLD activity in a $10 \mathrm{~mm}^{3}$ of cortex (a voxel) shows the consumption of oxygen of several hundreds of thousands of neurons. Therefore, in this thesis, we were limited to the top processing, i.e. the cognition, the behavioural processing or high-level dynamics of the brain. Then the challenge is to infer possible explanation of the sub-level (disrupted) activity. This is, probably, the next step neuro-engineer should focus on: how these large-scale dynamics have an impact on the neuronal substrate (topdown) or how they were formed by the neuronal substrate (bottom-up). To summarise, here, we have tried to analyze the top-down process, through (top) data-driven processing and modeling-from experiment towards theory-i.e. a decoding process/modeling of neural network behaviour. We did not regard the bottom-up modeling-from theory toward experiment, i.e. a coding/modeling of neural networks activity emergence process. If there were no limitations from the fMRI resolution, testing more biologically relevant dynamics giving rise to the emergence of the well-known brain waves, i.e at the micro-scale, could ensure consistency and could prove the higher-level dynamics correctness. EEG analyses and the brain prediction field of research should help in that regard.

This link between top-down and bottom-up models should be further explored. Perhaps, the Bayesian cognitive models are the most suitable for this (Kriegeskorte and Douglas, 2018). Finally, this integration model of levels of abstraction is not only needed at the biological and cognitive brain levels, but also at the neuroscientific research field one: "Brain science likewise needs to integrate its levels of description and to progress both bottom-up and top-down, so as to explain task performance on the basis of neuronal dynamics and provide a mechanistic account of how the brain gives rise to the mind" (Kriegeskorte and Douglas, 2018). This means we need, in neuroscience, more multidisciplinary top-down and bottom-up translational research.

\subsection{Future directions for neurodynamics in neuropsychiatric disorders}

\subsection{1 fMRI, preprocessing, and individual mapping}

As discussed in the previous section, the preprocessing pipeline plays a critical role in assessing brain dynamics using fMRI. The direct extraction of regions of interests (ROIs) or networks is not consensually defined. Here, in our small cohort studies, group-ICA decomposition seemed the most appropriate. However, if one needs to be able to extract reliably the presented dynamics metrics of one subject, single-subject ICA might not be the preferred method. If we aim at personalised medicine and treatment, other preprocessing pipelines for functional parcellation of the brain should be explored. Guideline for fMRI acquisition should also be agreed upon. Recent research projects actually try to tackle the abovementioned issues, through guideline and consensus of what and how data should be collected-with the ENIGMA consortium for MDD (Schmaal et al., 2017), for example. Thorough research on what can be the best functional brain parcellation technique for a more personalised biomarker analysis might also benefit from this consensus. One proposition is to have robust individual functional parcellation of brain back-projected from a detailed atlas (average brain functional maps from large representative dataset) as in (Wang et al., 2015). These guidelines on acquisition and processing of data could be done for many neuropsychiatric disorders, and might be disease-dependent. Indeed, perhaps, task-based fMRI should be 
preferred in case of ADHD for instance, where brain dynamics that occur during an attentionbased paradigm might turn out to be more relevant biomarkers-although we advocate the use of rs-fMRI throughout this thesis.

\subsubsection{Multidimensional and neuropsychiatric-specific biomarkers}

Seeking consensus in acquisition and processing of fMRI data leads us to another avenue neuropsychoradiology should aim at exploring: the multidimensional properties of the brain. Indeed, so far, we have tackled the issue of finding relevant biomarker for neuropsychiatric disorders solely at the fMRI level; with relatively high spatial resolution (in the order of few $\mathrm{mm}^{3}$ ), and low temporal resolutions (1-2 s per volume acquisition). However, as mentioned above, added information at finer spatial and temporal level-we can think of EEG for higher temporal resolutions-could greatly improve biomarkers validation. Combining techniques and disciplines, such as electronics, biology and genetics, and computer science should also lead to a better understanding of the (abnormal) brain functioning, since it would give us more specific pictures of its mechanisms.

In the field of psychoradiology, the revolution towards personalised medicine will probably not start from scratch. Previous research has provided us with prior knowledge on clear biomarkers of specific neuropsychiatric disorders: for instance, amyloid deposition and plaques are clear indicators of Alzheimer's disease (AD). Thus, as mentioned before, adding a new dimension, such as the white matter integrity to fMRI-based features, can help designing strong (multi-modal) biomarkers for AD. Similarly, in case of schizophrenia, time in specific brain states (Damaraju et al., 2014) and abnormalities in white matter integrity (Mamah et al., 2019; Pasternak et al., 2012) are two markers of the disease, and could be also combined to have a better multi-modal biomarker. In case of accelerated ageing, the DAN, DMN, and SN should be the first networks to be evaluated in their integrity/normal functioning in order to develop further reliable marker of the cognitive decline progression. Altogether, the search for improved biomarkers for mental disorders might be disease-specific - even though in case of psychiatric disorders, common roots of brain abnormality have been pointed out (Sprooten et al., 2017). Having more disease-specific markers should also help stratifying the disorders, in terms of severity or effectiveness of chosen treatment (i.e. probability of success of treatment response).

\subsubsection{Classifier, improved models and images}

An improvement of classification models and development of advanced machine learning algorithms should also be a focus for psychoradiology research. Indeed, as mentioned earlier, schizophrenia display multi-modal makers (white matter integrity with DTI, and dwelling time in brain state with fMRI). Hence to improve classification, a first step might be to use a decision-treebased algorithm on structural/physiological data, followed by an SVM classification on fMRIbased dynamics markers. This is just an example for one disease, but one can expect having multiple possibilities when it comes to other disorders. In case of sleep disorders for instance, to improve the model for multi-class classification of insomnia severity, one could think of, for instance, extracting an EEG mapping (during sleep) prior to an rs-fMRI scanning, and train multi-modal classifiers. This improvement of classification using multimodal analyses could, given enough data, have a prognostic benefit: either prognosis of the course of the symptoms or prediction of the positive effect of different treatments (using retrospective studies).

Regarding the fMRI technique, not only the software for post-processing can be improved, but also the hardware: new and more disease-specific sequences should be developed to increase the 
reliability of biomarkers; e.g. shorter TR traded off with grosser spatial resolution for finer temporal dynamics descriptions of a large network in the brain; or higher spatial resolution but in a more localised MRI, e.g. by scanning only slices of motor cortex for Parkinson, with higher resolution, without trading off the temporal resolution (slice acquisition speed. All these improvements can only be possible given enough data to train and test the validity of the methods. Data sharing project such as OASIS-brains ${ }^{2}, \mathrm{ABIDE}^{3}$, OpenNeuro ${ }^{4}$ or $\mathrm{HCP}^{5}$ make the access to MRI and fMRI data public and freely available. These are great initiatives for further research in psychoradiology, and the improvement of classification pipelines. Another novel technique for assessing at higher speed brain functioning changes using MRI, is the Magnetic Resonance Elastography (MRE), it assesses stiffness of cortical regions (Lan et al., 2020; Patz et al., 2019). This method is promising since it can acquire functional data (based on brain tissue stiffness) at substantially higher speed than traditional fMRI acquisition, without having to modify the hardware; and it can already be applied on 3T MRI scanners, which are common in most hospitals.

With regard to rs-fMRI post-processing and our brain dynamics models presented in this thesis, an interesting idea for improvement would be to evaluates these dynamics methods at different levels of abstractions, i.e. at different stage of the top-down and bottom-up processes, as mentioned in the previous section. We could, for example: (i) extract the global abnormal dynamics with EGN approach, expecting two or three networks to appear impaired; (ii) subscale the large-scale networks into multiple nodes to apply an effective connectivity and assess the within-network abnormality, or extract the most probable effective connectivity topography of the network with the Bayesian search methods; and (iii) evaluate time of coherence or other transient-related measures of the within- and between- network connectivity (that are relevant in (i) and (ii)). That would infer which large scale network are impaired (EGN), and which nodes are relevant in their directed connectivity (GC) and serve as modular nodes (transients in WCoh maps). The benefit of such pipeline is hypothetical, yet, a thorough analysis could tell us more about the mechanisms of action of neuropsychiatric disorders at different level of abstractions of brain dynamics; or at least this would show which methods are preferable for specific dynamic scales. Moreover, it is important that such a refined approach stays fully explainable; that way, it can be easily optimised with 'classic' machine-learning algorithms-for transparent computer-aided diagnostic tools.

Overall, neuroimaging research would benefit from improving the whole pipeline for extracting relevant biomarkers, from more disease-specific image acquisitions to more advanced final multimodal classification analysis. This would greatly contribute to the field of psychoradiology and its quest for more robust biomarkers of brain disorders.

\subsection{General conclusions}

Neuropsychiatric disorders are defined as neurological conditions with psychiatric symptoms, such as impairments in cognition. An important issue in these diseases is the lack of robust brain biomarkers, which causes diagnostic uncertainty and lack of effective treatments. Finding the underlying mechanism of action of these mental disorders can help designing diagnostic and prognostic tools that would alleviate this issue in neuropsychiatry. In this thesis, we aimed at investigating new techniques for extracting brain biomarkers of neuropsychiatric disorders. Finding these neurobiomarkers by means of neuroimaging techniques, such as functional MRI, is actually the core of psychoradiology. We contribute to this field by introducing pioneer analysis methods

\footnotetext{
${ }^{2}$ https://www .oasis-brains.org/\#data

${ }^{3}$ http://fcon_1000.projects.nitrc.org/indi/abide/

${ }^{4}$ https://openneuro.org/

${ }^{5}$ http://www . humanconnectomeproject.org/
} 
and showing the benefit of using brain dynamics from resting-state fMRI as biomarkers over the usual (static) functional connectivity that has been used for decades.

The neurodynamics between large-scale cognitive brain networks can be described using new promising models introduced in this thesis. The game theory on network approach applied on brain images has given us the best classification performances in case of accelerated cognitive ageing (ACA). Our novel wavelet-based time of in-phase coherence is also a good descriptor of neuropsychiatric disorders, such as ACA and autism spectrum disorder (ASD). The Granger causality between networks, on the other hand, falls short of being a relevant marker, albeit displaying clear abnormalities in the aforementioned mental disorders. Additionally, a Bayesian search approach was investigated, which has shown its advantage in describing the brain dynamics changes when study participants listened to Mozart's sonata K448.

Together with these advances we also see questions rising about the understanding and applications of these neurdoynamics. For instance, wavelet-coherence maps might reveal other useful descriptors of inter-network dynamics, such as transient effects in the pairwise coherent connectivity and their influence on modulating network dynamics; and new wavelet coherence-based derived metrics must be further investigated. Measures of effective connectivity (such as Granger causality) might also be more insightful if it is applied within the brain networks, involving a greater number of nodes in the network analysis; or could even be deployed at the voxel level of fMRI BOLD signals. Overall, combining these dynamics brain features can greatly help diagnosing neuropsychiatric disorders.

Our contribution to the field of psychoradiology is threefold: (i) we introduce three pioneer methods to describe brain dynamics and validate them in clinical research; (ii) brain dynamics evaluation of neuropsychiatric patients, in terms of wavelet coherence and emulative power, has a clear added value over the usual static functional connectivity-at least for ACA and ASD; (iii) a new way of measuring the effective connectivity between brain networks, i.e. the Bayesian approach, shows high potential for describing brain dynamics and must be further investigated for neuropsychiatric biomarkers.

This work is at its infant stage, and modestly sheds light on the mechanisms of action of the two aforementioned neuropsychiatric disorders: ACA and ASD. The classification pipeline presented in this thesis could be refined by disease-specific multimodal approaches or by combining neurodynamics metrics at various granularity levels of brain (from neurons to networks). If this pipeline is also refined and tested on larger heterogeneous and multisite datasets of rs-fMRI, the dynamics markers, presented here, could have great predictive values in neuropsychiatry research. Indeed, this could help designing better treatments and prognostic tools-for disease progression or treatment response-for mental disorders. Therefore, this further research will considerably contribute to the field of psychoradiology, and its quest for robust image-based neurobiomarkers. 



\section{Appendix A}

\section{Appendix Chapter 4}

\section{A.1 Multivariate Conditional Granger Causality theory}

We use a multivariate Granger causality MATLAB toolbox to calculate the conditional granger causality magnitudes. Algorithms and functions of this toolbox are describe in Lionel Barnett's paper (Barnett and Seth, 2014). The following parts describe the mathematical theory and processes needed behind Granger causality. Geweke (Geweke, 1984) describes the measure of the Granger causality as follows:

$$
F_{\mathbf{Y} \rightarrow \mathbf{X}} \equiv \ln \frac{\left|\Sigma_{x x}^{\prime}\right|}{\left|\Sigma_{x x}\right|}
$$

In this equation, $\Sigma_{x x}^{\prime}$ is variance of the prediction error (residuals) of $\mathbf{X}_{t}$ excluding the possible causal influence of $\mathbf{Y}_{t}$, and $\Sigma_{x x}$ is variance of the prediction error of $\mathbf{X}_{t}$ including the possible causal influence of $\mathbf{Y}_{t}$. To measure these variances, the vector autoregressive model (VAR) is applied (Deshpande, Santhanam, and Hu, 2011; Roebroeck, Formisano, and Goebel, 2005; Zhou et al., 2011). This model is defined as

$$
\mathbf{U}_{t}=\sum_{k=1}^{p} A_{k} \cdot \mathbf{U}_{t-k}+\varepsilon_{t}
$$

here, $\boldsymbol{U}_{t}$ is a time series, $A_{k}$ - regression coefficients, $\varepsilon$ - the residuals and $p$ - the model order representing the number of past lags taken into account. This model order is calculated from time series using the Akaike Information Criterion (AIC) (Akaike, 1974; Deshpande et al., 2009). If $\boldsymbol{U}_{t}$ is a multivariate vector composed if two time series $X$ and $Y$, the VAR model can be developed as shown in Eq. (A.4).

$$
\begin{aligned}
& \mathbf{U}_{t}=\left(\begin{array}{l}
\mathbf{X}_{t} \\
\mathbf{Y}_{t}
\end{array}\right) \\
& \left(\begin{array}{l}
\mathbf{X}_{t} \\
\mathbf{Y}_{t}
\end{array}\right)=\sum_{k=1}^{p}\left(\begin{array}{ll}
A_{x x, k} & A_{x y, k} \\
A_{y x, k} & A_{y y, k}
\end{array}\right)\left(\begin{array}{l}
\mathbf{X}_{t-k} \\
\mathbf{Y}_{t-k}
\end{array}\right)+\left(\begin{array}{l}
\varepsilon_{x, t} \\
\varepsilon_{y, t}
\end{array}\right)
\end{aligned}
$$

with the covariance matrix of residuals expressed as

$$
\Sigma \equiv \operatorname{cov}\left(\begin{array}{c}
\varepsilon_{x, t} \\
\varepsilon_{y, t}
\end{array}\right)=\left(\begin{array}{cc}
\Sigma_{x x} & \Sigma_{x y} \\
\Sigma_{y x} & \Sigma_{y y}
\end{array}\right)
$$

By applying the Morf's variant of the Locally Weighted Regression (LWR) algorithm (Morf et al., 1978), which is very efficient for likelihood-based model selection such as AIC (McQuarrie 
and Tsai, 1998), we estimate the VAR parameters. If we consider the X-component of the regression (A.4), we obtain the following expression:

$$
\mathbf{X}_{t}=\sum_{k=1}^{p} A_{x x, k} \cdot \mathbf{X}_{t-k}+\sum_{k=1}^{p} A_{x y, k} \cdot \mathbf{Y}_{t-k}+\boldsymbol{\varepsilon}_{x, t}
$$

and if we suppose no conditional dependence between $\mathbf{X}$ and the past of $\mathbf{Y}\left(A_{x y, 1}=A_{x y, 2}=\ldots=\right.$ $\left.A_{x y, p}=0\right)$, we obtain the reduced regression:

$$
\mathbf{X}_{t}=\sum_{k=1}^{p} A_{x x, k}^{\prime} \cdot \mathbf{X}_{t-k}+\varepsilon_{x, t}^{\prime}
$$

We finally get from (A.5) the following: $\Sigma_{x x}=\operatorname{cov}\left(\varepsilon_{x, t}\right)$ and $\Sigma_{x x}^{\prime}=\operatorname{cov}\left(\varepsilon_{x, t}^{\prime}\right)$. Hence, we get the Geweke measure of the Granger causality $F_{\mathbf{Y} \rightarrow \mathbf{X}}$ (A.1).

In our case, we have more than two time series, thus the Granger causality between one signal and another is depending on the information added by the past of the other signals. Let us now illustrate this conditional pairwise G-causality. Suppose we have

$$
\mathbf{U}_{t}=\left(\begin{array}{c}
\mathbf{X}_{t} \\
\mathbf{Y}_{t} \\
\mathbf{Z}_{t}
\end{array}\right)
$$

where $\mathbf{Z}_{t}$ is a third set of variables. We aim at eliminating any joint effect of $\mathbf{Z}$ on the inference of the G-causality $\mathbf{Y}$ to $\mathbf{X}$. Thus, analogously to (A.4), the full and reduced regression for the $X$-component becomes respectively

$$
\mathbf{X}_{t}=\sum_{k=1}^{p} A_{x x, k} \cdot \mathbf{X}_{t-k}+\sum_{k=1}^{p} A_{x y, k} \cdot \mathbf{Y}_{t-k}+\sum_{k=1}^{p} A_{x z, k} \cdot \mathbf{Z}_{t-k}+\varepsilon_{x, t}
$$

and

$$
\mathbf{X}_{t}=\sum_{k=1}^{p} A_{x x, k}^{\prime} \cdot X_{t-k}+\sum_{k=1}^{p} A_{x z, k}^{\prime} \cdot \mathbf{Z}_{t-k}+\varepsilon_{x, t}^{\prime}
$$

which corresponds to (A.6) and (A.7) but with the inclusion of conditioning variables $\mathbf{Z}$ in both regressions. We can finally quantify the conditional G-causality as the log-likelihood ratio:

$$
F_{\mathbf{Y} \rightarrow \mathbf{X} \mid \mathbf{Z}} \equiv \ln \frac{\left|\Sigma_{x x}^{\prime}\right|}{\left|\Sigma_{x x}\right|}
$$

which can be defined as "the degree to which the past of $\mathbf{Y}$ helps predict $\mathbf{X}$ over and above the degree of which $\mathbf{X}$ is already predicted by its own past and the past of $\mathbf{Z}$ " (Barnett and Seth, 2014). 


\section{A.2 Supporting information of no between-group difference in spatial RSN connectivity}

TABLE A.1: Smallest FWE-corrected p-values in voxels showing a between-group RSN spatial difference.

\begin{tabular}{|l|l|l|l|l|}
\hline Network & \multicolumn{4}{|c|}{ p-value (corrected) } \\
\hline & Con $>$ ASD & $A S D>$ Con & Con $>$ ASD adj* & ASD $>{\text { Con } \text { adj }^{*}}^{*}$ \\
\hline Default-mode & 0.93 & 0.54 & 0.85 & 0.58 \\
\hline Fronto-parietal R & 0.94 & 0.13 & 0.59 & 0.23 \\
\hline Salience-executive & 0.39 & 0.36 & 0.65 & 0.66 \\
\hline Visual system & 0.33 & 0.83 & 0.38 & 0.74 \\
\hline Fronto-parietal L & 0.29 & 0.60 & 0.10 & 0.38 \\
\hline Auditory system & 0.97 & 0.59 & 0.79 & 0.61 \\
\hline Precuneus & 0.39 & 0.94 & 0.13 & 0.82 \\
\hline Sensorimotor 1 & 0.20 & 0.71 & 0.60 & 0.43 \\
\hline Sensorimotor 2 & 0.55 & 0.21 & 0.78 & 0.18 \\
\hline Ventral attention & 0.12 & 0.90 & 0.26 & 0.96 \\
\hline Cerebellum & 0.77 & 0.76 & 0.78 & 0.76 \\
\hline
\end{tabular}

No voxels survived at the threshold $P=0.05$. P-values are FWE-corrected for multiple comparison. adj*: statistical maps adjusted for subjects' ages, IQ levels and gray matter volumes. 


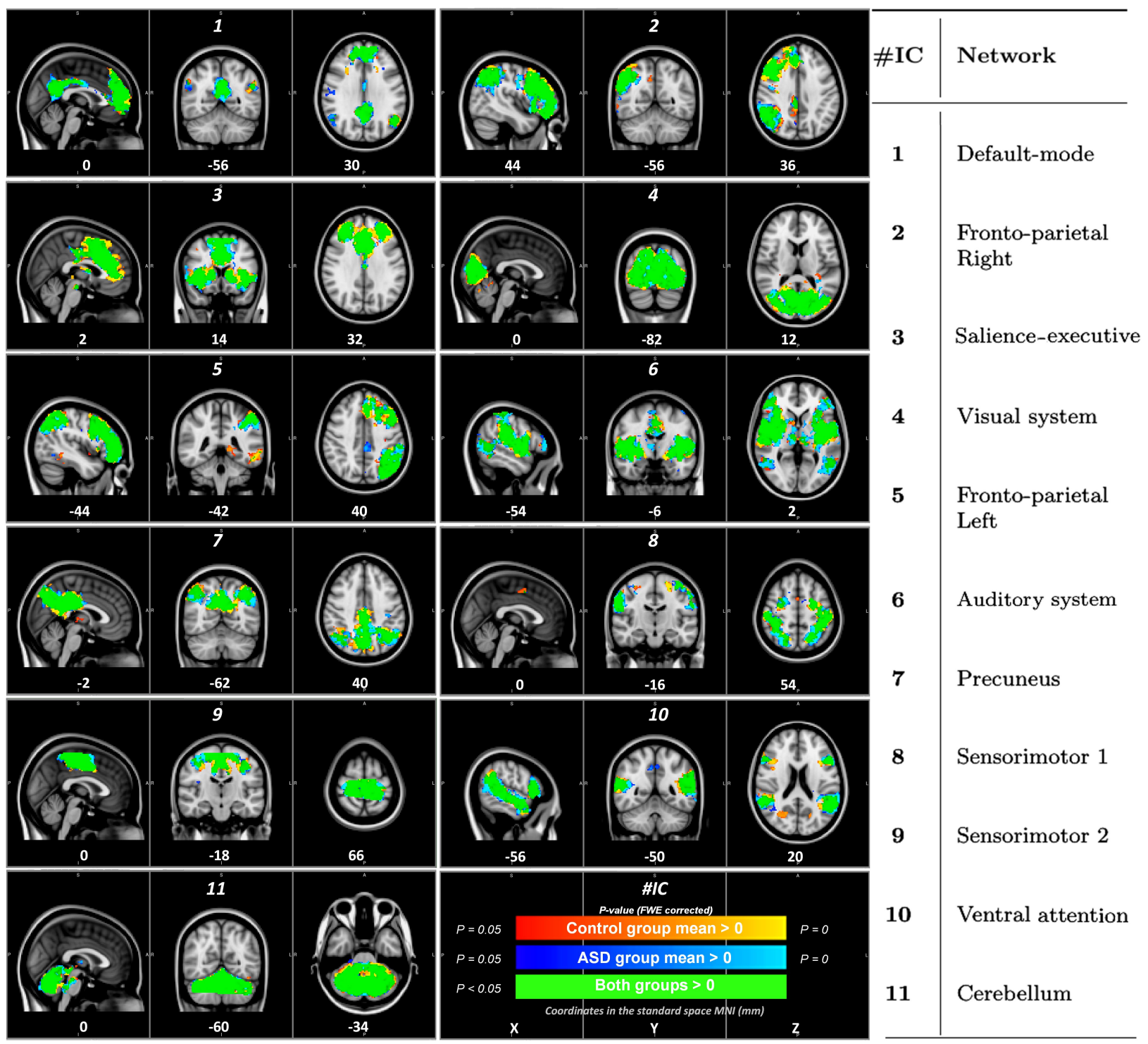

FIGURE A.1: Group mean effect per network. Red-yellow = Control group; Blue-lightblue = ASD group; Green $=$ Overlapping of both groups; thresholded at $p<0.05$ FWE corrected.

\section{A.3 1-back task fMRI protocol}

\section{A.3.1 1-back Task description}

The 1-back task looks at processing complex stimuli: social and non-social. The non-social complex stimuli are homes, the social complex stimuli are faces. The participant should not remember one specific house / face, but consider whether two photos in a row are exactly the same. The participant should remember, not only the identity of the house / face, but also the specific feature (house: upper or lower adjusted / face: emotion angry, happy, fear). Participant is instructed to press the "yes" button if the picture he/she are seeing is an exact copy of the previous one, and press the "no" button otherwise. Here an example (Figure A.2): Sequentially (from left to right) the pictures are displayed as below. Red dots means what the participant should respond when he/she sees that very picture. The task alternates randomly between the houses and the faces (see next paragraph). 

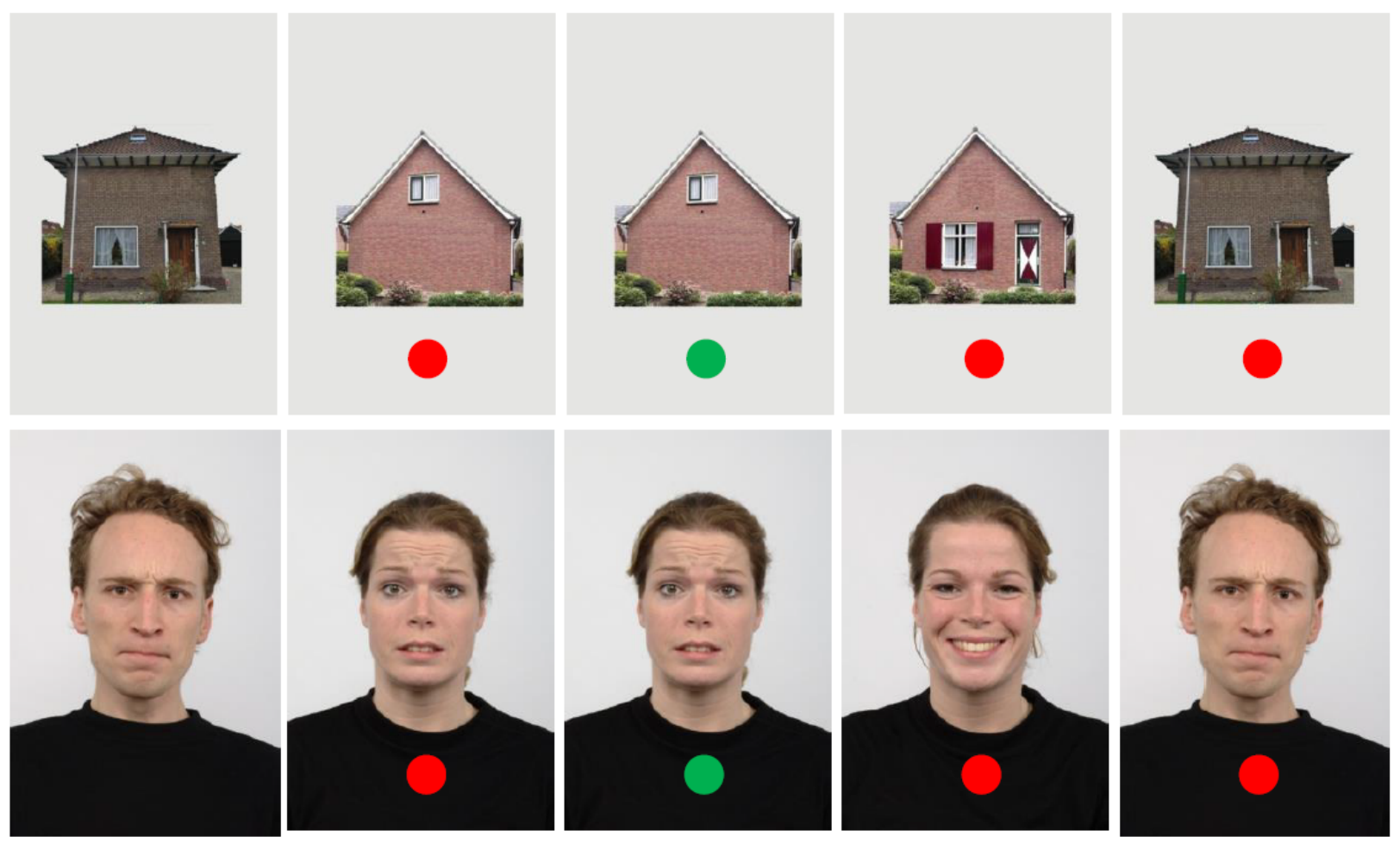

FIGURE A.2: Images displayed for n-back visual task in ASD study. red dot = participant should press 'no'; green dot $=$ participant should press 'yes'.

\section{A.3.2 Task procedure}

Before the participant goes into the scanner, the task will be practiced outside the scanner. The participant has already had extensive instructions (and practiced with the task) outside the scanner. The instructions in the scanner must therefore be limited to the literal instructions of the task as shown on the screen. - During the anatomical scan, the participant can read the instructions on screen again. - Before starting the fMRI scan: Read the instructions on the screen literally with the participant and see if he / she has any questions. - Start the fMRI scan if the participant does not have any questions the fMRI scan. The task will then automatically start and run with the scan. Note: If the participants wants feedback: Indicate that you cannot give it now because everything needs to be analyzed first and only if a group of people has been tested.

\section{A.3.3 Task design}

Below (Figure A.3), the schedule for displaying the picture used for the 1-back visual task (1minute period and at $50 \%$ task duty cycle). 


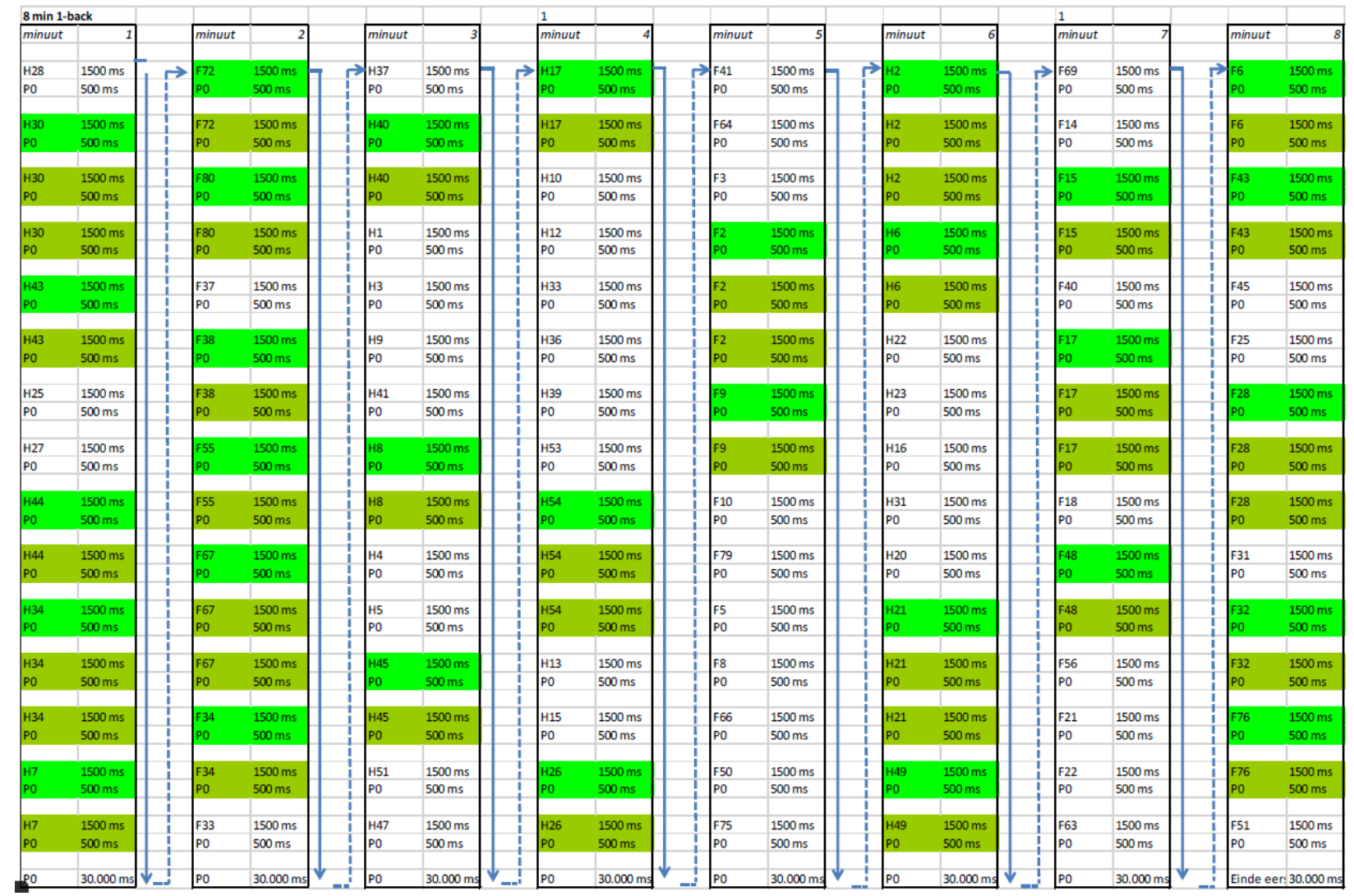

FigURE A.3: Design of the 1-back visual task. $\mathrm{H}=$ House picture; $\mathrm{F}=$ Face picture; $\mathrm{P} 0=$ Rest. 


\section{Appendix B}

\section{Appendix Chapter 5}

\section{B.1 Results of all classifier tested in setup 1 (4 features)}

Tables B.1- B. 3 depict the classifiers performance in the $1^{\text {st }}$ setup, i.e. using 4 features of in-phase time of coherence used: VENT-AUDI, VENT-VISU, VENT-FPL, VENT-FPR, for the LDA, SVMpolynomial and SVM-rbf respectively. 
TABLE B.1: Classification performances at all subscales with LDA (setup $1=4$ features).

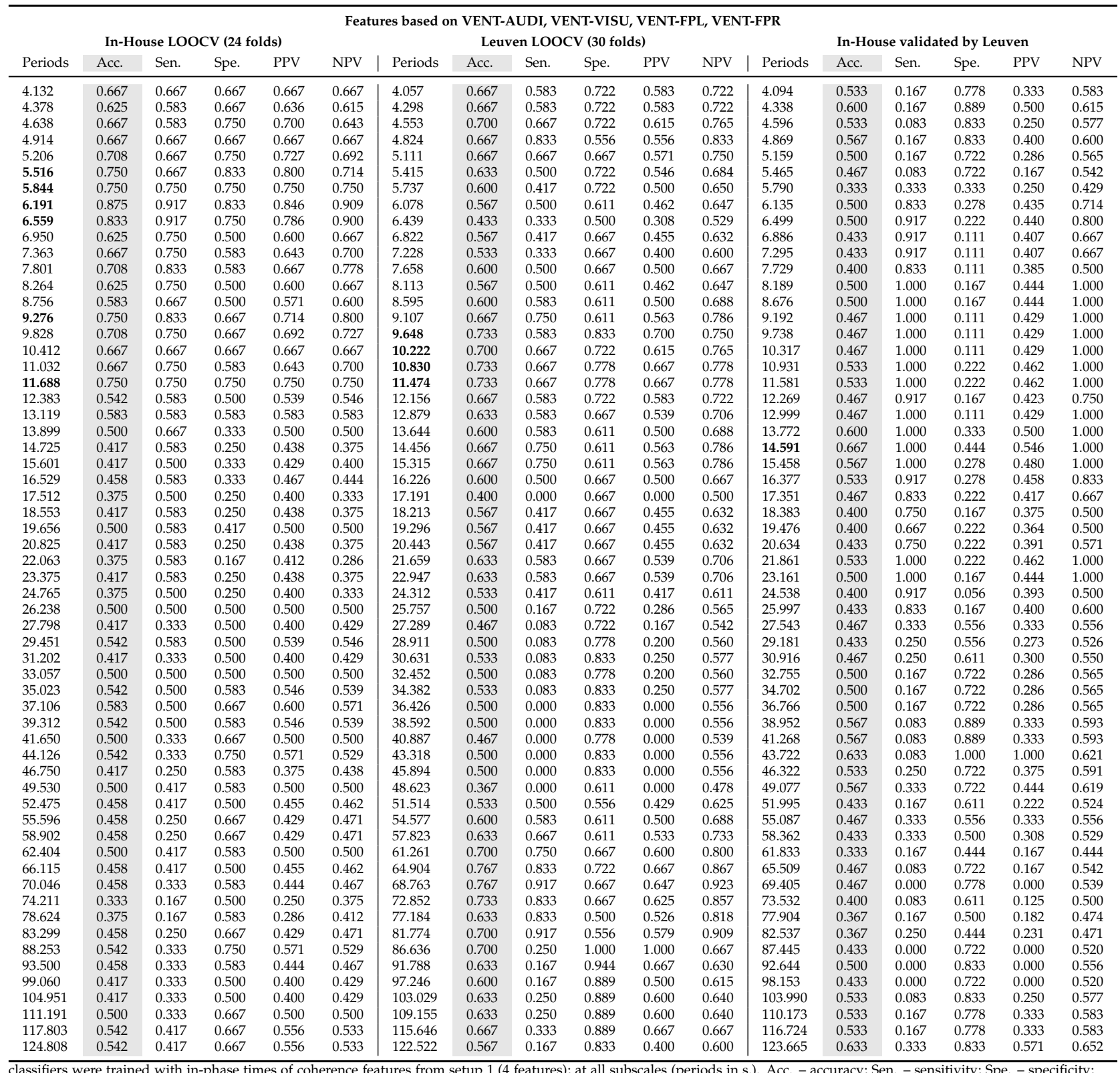

classifiers were trained with in-phase times of coherence features from setup 1 (4 features); at all subscales (periods in s.). Acc. - accuracy; Sen. - sensitivity; Spe. - specificity;
PPV - positive predictive value; NPV - negative predictive value; LOOVC - leave-one-out cross validatio; in bold - ranges of periods at which the classifiers performed the best (= results shown in Table 5.2). 
TABLE B.2: Classification performances at all subscales with SVM polynomial kernel (setup $1=4$ features).

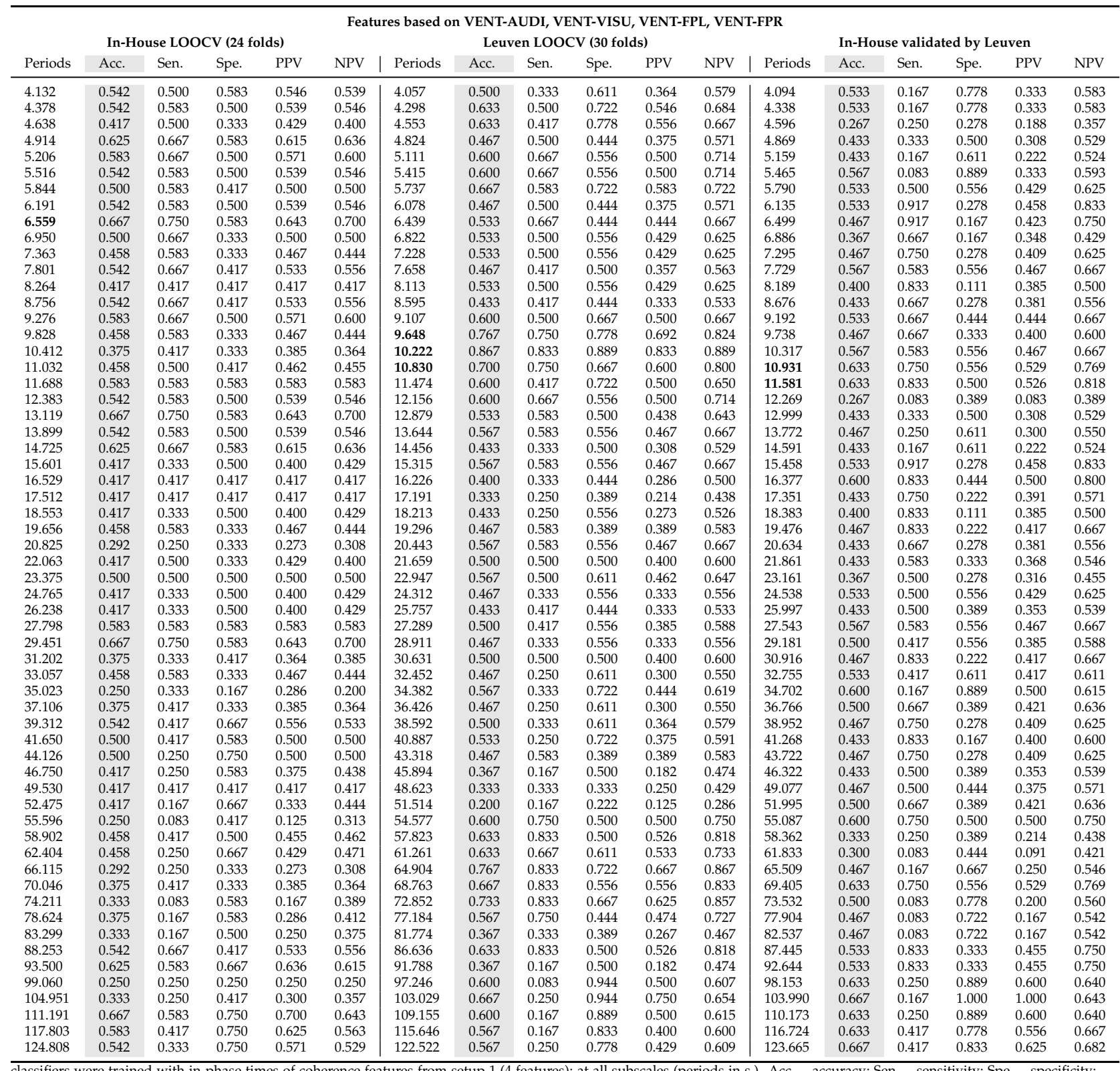
classifiers were trained with in-phase times of coherence features from setup 1 (4 features); at all subscales (periods in s.). Acc. - accuracy; Sen. - sensitivity; Spe. - specificity;
PPV - positive predictive value; NPV - negative predictive value; LOOVC - leave-one-out cross validation; in bold - ranges of periods at which the classifiers performed the best (= results shown in Table 5.2). 
TABLE B.3: Classification performances at all subscales with SVM rbf kernel (setup $1=4$ features).

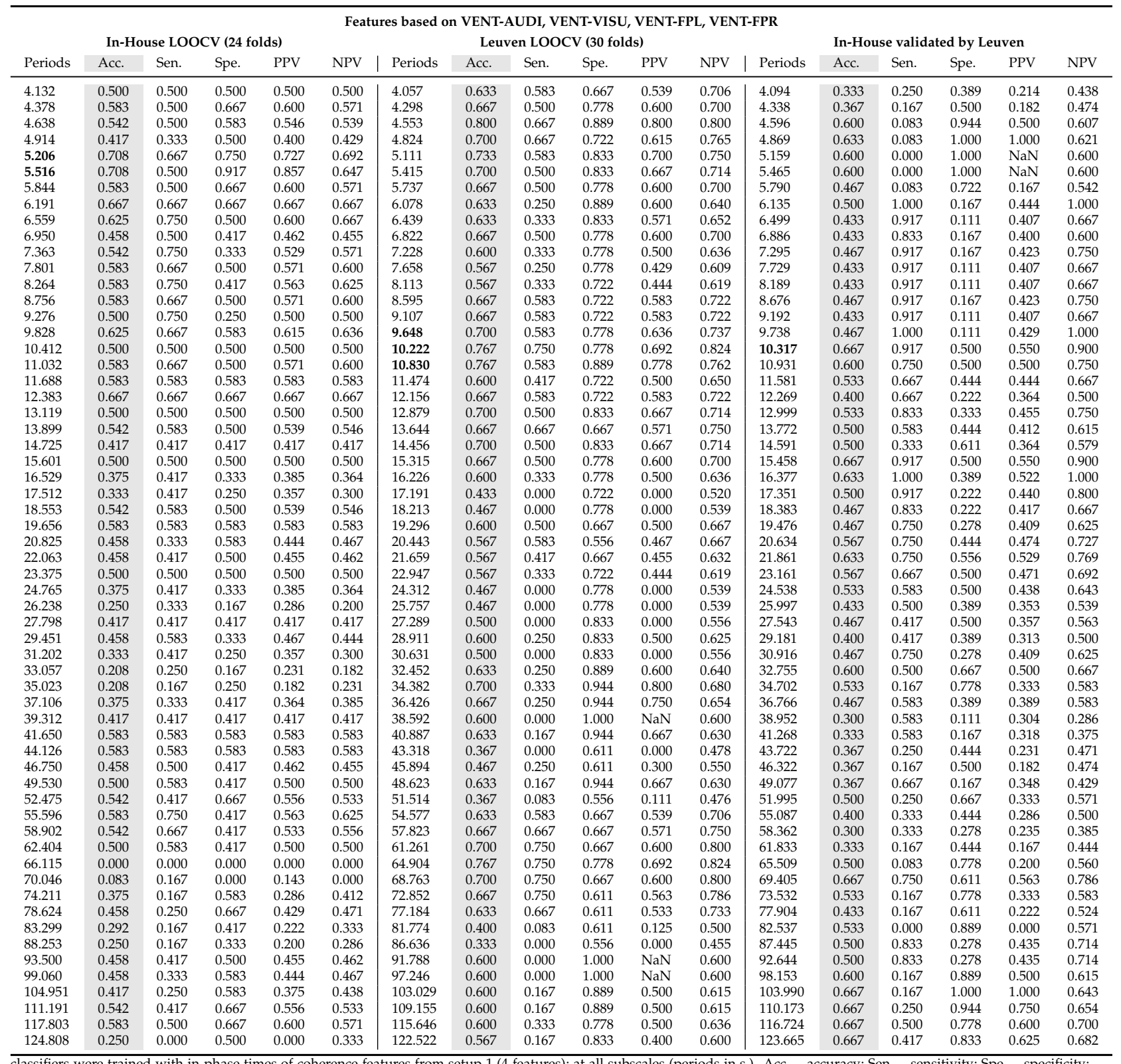

classifiers were trained with in-phase times of coherence features from setup 1 (4 features); at all subscales (periods in s.). Acc. - accuracy; Sen. - sensitivity; Spe. - specificity;
PPV - positive predictive value; NPV - negative predictive value; LOOVC - leave-one-out cross validation; in bold - ranges of periods at which the classifiers performed the best

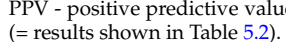




\section{B.2 Results of all classifier tested in Setup 2 ( 2 features)}

Tables B.4- B.6 depict the classifiers performance in the $2^{\text {nd }}$ setup, i.e. using 2 features of inphase time of coherence: VENT-FPL, VENT-FPR, for the LDA, SVM-polynomial and SVM-rbf respectively. 
TABLE B.4: Classification performances at all subscales with LDA (setup $2=2$ features).

\begin{tabular}{|c|c|c|c|c|c|c|c|c|c|c|c|c|c|c|c|c|c|}
\hline \multicolumn{18}{|c|}{ Features based on VENT-FPL, VENT-FPR } \\
\hline \multicolumn{6}{|c|}{ In-House LOOCV (24 folds) } & \multicolumn{6}{|c|}{ Leuven LOOCV (30 folds) } & \multicolumn{6}{|c|}{ In-House validated by Leuven } \\
\hline Periods & Acc. & Sen. & Spe. & PPV & NPV & Periods & Acc. & Sen. & Spe. & PPV & NPV & Periods & Acc. & Sen. & Spe. & PPV & NPV \\
\hline 4.132 & 0.708 & 0.667 & 0.750 & 0.727 & 0.692 & 4.057 & 0.567 & 0.000 & 0.944 & 0.000 & 0.586 & 4.094 & 0.533 & 0.167 & 0.778 & 0.333 & 0.583 \\
\hline 4.378 & 0.667 & 0.667 & 0.667 & 0.667 & 0.667 & 4.298 & 0.567 & 0.000 & 0.944 & 0.000 & 0.586 & 4.338 & 0.567 & 0.167 & 0.833 & 0.400 & 0.600 \\
\hline 4.638 & 0.583 & 0.500 & 0.667 & 0.600 & 0.571 & 4.553 & 0.500 & 0.000 & 0.833 & 0.000 & 0.556 & 4.596 & 0.533 & 0.167 & 0.778 & 0.333 & 0.583 \\
\hline 4.914 & 0.542 & 0.500 & 0.583 & 0.546 & 0.539 & 4.824 & 0.600 & 0.333 & 0.778 & 0.500 & 0.636 & 4.869 & 0.467 & 0.167 & 0.667 & 0.250 & 0.546 \\
\hline 5.206 & 0.458 & 0.417 & 0.500 & 0.455 & 0.462 & 5.111 & 0.600 & 0.333 & 0.778 & 0.500 & 0.636 & 5.159 & 0.367 & 0.667 & 0.167 & 0.348 & 0.429 \\
\hline 5.516 & 0.583 & 0.500 & 0.667 & 0.600 & 0.571 & 5.415 & 0.667 & 0.417 & 0.833 & 0.625 & 0.682 & 5.465 & 0.267 & 0.667 & 0.000 & 0.308 & 0.000 \\
\hline 5.844 & 0.625 & 0.500 & 0.750 & 0.667 & 0.600 & 5.737 & 0.633 & 0.417 & 0.778 & 0.556 & 0.667 & 5.790 & 0.333 & 0.833 & 0.000 & 0.357 & 0.000 \\
\hline 6.191 & 0.500 & 0.500 & 0.500 & 0.500 & 0.500 & 6.078 & 0.500 & 0.000 & 0.833 & 0.000 & 0.556 & 6.135 & 0.400 & 1.000 & 0.000 & 0.400 & $\mathrm{NaN}$ \\
\hline 6.559 & 0.500 & 0.667 & 0.333 & 0.500 & 0.500 & 6.439 & 0.567 & 0.000 & 0.944 & 0.000 & 0.586 & 6.499 & 0.433 & 1.000 & 0.056 & 0.414 & 1.000 \\
\hline 6.950 & 0.542 & 0.667 & 0.417 & 0.533 & 0.556 & 6.822 & 0.600 & 0.000 & 1.000 & $\mathrm{NaN}$ & 0.600 & 6.886 & 0.433 & 1.000 & 0.056 & 0.414 & 1.000 \\
\hline 7.363 & 0.542 & 0.667 & 0.417 & 0.533 & 0.556 & 7.228 & 0.467 & 0.000 & 0.778 & 0.000 & 0.539 & 7.295 & 0.433 & 1.000 & 0.056 & 0.414 & 1.000 \\
\hline 7.801 & 0.500 & 0.583 & 0.417 & 0.500 & 0.500 & 7.658 & 0.567 & 0.250 & 0.778 & 0.429 & 0.609 & 7.729 & 0.433 & 1.000 & 0.056 & 0.414 & 1.000 \\
\hline 8.264 & 0.542 & 0.667 & 0.417 & 0.533 & 0.556 & 8.113 & 0.667 & 0.500 & 0.778 & 0.600 & 0.700 & 8.189 & 0.467 & 1.000 & 0.111 & 0.429 & 1.000 \\
\hline 8.756 & 0.542 & 0.583 & 0.500 & 0.539 & 0.546 & 8.595 & 0.733 & 0.667 & 0.778 & 0.667 & 0.778 & 8.676 & 0.467 & 1.000 & 0.111 & 0.429 & 1.000 \\
\hline 9.276 & 0.542 & 0.583 & 0.500 & 0.539 & 0.546 & 9.107 & 0.667 & 0.667 & 0.667 & 0.571 & 0.750 & 9.192 & 0.467 & 1.000 & 0.111 & 0.429 & 1.000 \\
\hline 9.828 & 0.625 & 0.750 & 0.500 & 0.600 & 0.667 & 9.648 & 0.733 & 0.750 & 0.722 & 0.643 & 0.813 & 9.738 & 0.500 & 1.000 & 0.167 & 0.444 & 1.000 \\
\hline 10.412 & 0.750 & 0.833 & 0.667 & 0.714 & 0.800 & 10.222 & 0.833 & 0.917 & 0.778 & 0.733 & 0.933 & 10.317 & 0.567 & 1.000 & 0.278 & 0.480 & 1.000 \\
\hline 11.032 & 0.708 & 0.833 & 0.583 & 0.667 & 0.778 & 10.830 & 0.767 & 0.750 & 0.778 & 0.692 & 0.824 & 10.931 & 0.467 & 0.917 & 0.167 & 0.423 & 0.750 \\
\hline 11.688 & 0.708 & 0.750 & 0.667 & 0.692 & 0.727 & 11.474 & 0.700 & 0.667 & 0.722 & 0.615 & 0.765 & 11.581 & 0.500 & 0.917 & 0.222 & 0.440 & 0.800 \\
\hline 12.383 & 0.750 & 0.750 & 0.750 & 0.750 & 0.750 & 12.156 & 0.667 & 0.750 & 0.611 & 0.563 & 0.786 & 12.269 & 0.500 & 0.917 & 0.222 & 0.440 & 0.800 \\
\hline 13.119 & 0.667 & 0.667 & 0.667 & 0.667 & 0.667 & 12.879 & 0.633 & 0.667 & 0.611 & 0.533 & 0.733 & 12.999 & 0.467 & 1.000 & 0.111 & 0.429 & 1.000 \\
\hline 13.899 & 0.667 & 0.833 & 0.500 & 0.625 & 0.750 & 13.644 & 0.600 & 0.667 & 0.556 & 0.500 & 0.714 & 13.772 & 0.567 & 0.917 & 0.333 & 0.478 & 0.857 \\
\hline 14.725 & 0.667 & 0.833 & 0.500 & 0.625 & 0.750 & 14.456 & 0.633 & 0.583 & 0.667 & 0.539 & 0.706 & 14.591 & 0.567 & 1.000 & 0.278 & 0.480 & 1.000 \\
\hline 15.601 & 0.500 & 0.667 & 0.333 & 0.500 & 0.500 & 15.315 & 0.767 & 0.667 & 0.833 & 0.727 & 0.790 & 15.458 & 0.533 & 1.000 & 0.222 & 0.462 & 1.000 \\
\hline 16.529 & 0.542 & 0.750 & 0.333 & 0.529 & 0.571 & 16.226 & 0.700 & 0.500 & 0.833 & 0.667 & 0.714 & 16.377 & 0.467 & 0.917 & 0.167 & 0.423 & 0.750 \\
\hline 17.512 & 0.417 & 0.500 & 0.333 & 0.429 & 0.400 & 17.191 & 0.567 & 0.000 & 0.944 & 0.000 & 0.586 & 17.351 & 0.433 & 0.917 & 0.111 & 0.407 & 0.667 \\
\hline 18.553 & 0.458 & 0.583 & 0.333 & 0.467 & 0.444 & 18.213 & 0.400 & 0.000 & 0.667 & 0.000 & 0.500 & 18.383 & 0.400 & 0.833 & 0.111 & 0.385 & 0.500 \\
\hline 19.656 & 0.375 & 0.500 & 0.250 & 0.400 & 0.333 & 19.296 & 0.600 & 0.500 & 0.667 & 0.500 & 0.667 & 19.476 & 0.467 & 0.917 & 0.167 & 0.423 & 0.750 \\
\hline 20.825 & 0.417 & 0.583 & 0.250 & 0.438 & 0.375 & 20.443 & 0.500 & 0.333 & 0.611 & 0.364 & 0.579 & 20.634 & 0.400 & 0.833 & 0.111 & 0.385 & 0.500 \\
\hline 22.063 & 0.458 & 0.667 & 0.250 & 0.471 & 0.429 & 21.659 & 0.567 & 0.500 & 0.611 & 0.462 & 0.647 & 21.861 & 0.633 & 0.917 & 0.444 & 0.524 & 0.889 \\
\hline 23.375 & 0.458 & 0.667 & 0.250 & 0.471 & 0.429 & 22.947 & 0.700 & 0.750 & 0.667 & 0.600 & 0.800 & 23.161 & 0.567 & 1.000 & 0.278 & 0.480 & 1.000 \\
\hline 24.765 & 0.458 & 0.667 & 0.250 & 0.471 & 0.429 & 24.312 & 0.500 & 0.250 & 0.667 & 0.333 & 0.571 & 24.538 & 0.500 & 0.833 & 0.278 & 0.435 & 0.714 \\
\hline 26.238 & 0.083 & 0.083 & 0.083 & 0.083 & 0.083 & 25.757 & 0.633 & 0.250 & 0.889 & 0.600 & 0.640 & 25.997 & 0.400 & 0.667 & 0.222 & 0.364 & 0.500 \\
\hline 27.798 & 0.292 & 0.167 & 0.417 & 0.222 & 0.333 & 27.289 & 0.533 & 0.000 & 0.889 & 0.000 & 0.571 & 27.543 & 0.300 & 0.583 & 0.111 & 0.304 & 0.286 \\
\hline 29.451 & 0.500 & 0.667 & 0.333 & 0.500 & 0.500 & 28.911 & 0.533 & 0.083 & 0.833 & 0.250 & 0.577 & 29.181 & 0.333 & 0.667 & 0.111 & 0.333 & 0.333 \\
\hline 31.202 & 0.292 & 0.333 & 0.250 & 0.308 & 0.273 & 30.631 & 0.600 & 0.167 & 0.889 & 0.500 & 0.615 & 30.916 & 0.533 & 0.333 & 0.667 & 0.400 & 0.600 \\
\hline 33.057 & 0.542 & 0.500 & 0.583 & 0.546 & 0.539 & 32.452 & 0.600 & 0.167 & 0.889 & 0.500 & 0.615 & 32.755 & 0.533 & 0.333 & 0.667 & 0.400 & 0.600 \\
\hline 35.023 & 0.583 & 0.583 & 0.583 & 0.583 & 0.583 & 34.382 & 0.633 & 0.167 & 0.944 & 0.667 & 0.630 & 34.702 & 0.533 & 0.083 & 0.833 & 0.250 & 0.577 \\
\hline 37.106 & 0.542 & 0.500 & 0.583 & 0.546 & 0.539 & 36.426 & 0.567 & 0.000 & 0.944 & 0.000 & 0.586 & 36.766 & 0.500 & 0.000 & 0.833 & 0.000 & 0.556 \\
\hline 39.312 & 0.667 & 0.583 & 0.750 & 0.700 & 0.643 & 38.592 & 0.567 & 0.000 & 0.944 & 0.000 & 0.586 & 38.952 & 0.533 & 0.000 & 0.889 & 0.000 & 0.571 \\
\hline 41.650 & 0.625 & 0.500 & 0.750 & 0.667 & 0.600 & 40.887 & 0.533 & 0.000 & 0.889 & 0.000 & 0.571 & 41.268 & 0.600 & 0.083 & 0.944 & 0.500 & 0.607 \\
\hline 44.126 & 0.583 & 0.417 & 0.750 & 0.625 & 0.563 & 43.318 & 0.533 & 0.000 & 0.889 & 0.000 & 0.571 & 43.722 & 0.633 & 0.083 & 1.000 & 1.000 & 0.621 \\
\hline 46.750 & 0.583 & 0.417 & 0.750 & 0.625 & 0.563 & 45.894 & 0.533 & 0.000 & 0.889 & 0.000 & 0.571 & 46.322 & 0.600 & 0.250 & 0.833 & 0.500 & 0.625 \\
\hline 49.530 & 0.542 & 0.417 & 0.667 & 0.556 & 0.533 & 48.623 & 0.567 & 0.000 & 0.944 & 0.000 & 0.586 & 49.077 & 0.500 & 0.250 & 0.667 & 0.333 & 0.571 \\
\hline 52.475 & 0.583 & 0.500 & 0.667 & 0.600 & 0.571 & 51.514 & 0.533 & 0.000 & 0.889 & 0.000 & 0.571 & 51.995 & 0.467 & 0.167 & 0.667 & 0.250 & 0.546 \\
\hline 55.596 & 0.583 & 0.417 & 0.750 & 0.625 & 0.563 & 54.577 & 0.567 & 0.000 & 0.944 & 0.000 & 0.586 & 55.087 & 0.433 & 0.167 & 0.611 & 0.222 & 0.524 \\
\hline 58.902 & 0.625 & 0.417 & 0.833 & 0.714 & 0.588 & 57.823 & 0.500 & 0.000 & 0.833 & 0.000 & 0.556 & 58.362 & 0.433 & 0.167 & 0.611 & 0.222 & 0.524 \\
\hline 62.404 & 0.542 & 0.417 & 0.667 & 0.556 & 0.533 & 61.261 & 0.533 & 0.000 & 0.889 & 0.000 & 0.571 & 61.833 & 0.400 & 0.083 & 0.611 & 0.125 & 0.500 \\
\hline 66.115 & 0.542 & 0.417 & 0.667 & 0.556 & 0.533 & 64.904 & 0.600 & 0.083 & 0.944 & 0.500 & 0.607 & 65.509 & 0.467 & 0.083 & 0.722 & 0.167 & 0.542 \\
\hline 70.046 & 0.500 & 0.333 & 0.667 & 0.500 & 0.500 & 68.763 & 0.600 & 0.083 & 0.944 & 0.500 & 0.607 & 69.405 & 0.500 & 0.000 & 0.833 & 0.000 & 0.556 \\
\hline 74.211 & 0.458 & 0.333 & 0.583 & 0.444 & 0.467 & 72.852 & 0.633 & 0.167 & 0.944 & 0.667 & 0.630 & 73.532 & 0.500 & 0.000 & 0.833 & 0.000 & 0.556 \\
\hline 78.624 & 0.500 & 0.333 & 0.667 & 0.500 & 0.500 & 77.184 & 0.633 & 0.167 & 0.944 & 0.667 & 0.630 & 77.904 & 0.500 & 0.000 & 0.833 & 0.000 & 0.556 \\
\hline 83.299 & 0.458 & 0.250 & 0.667 & 0.429 & 0.471 & 81.774 & 0.633 & 0.167 & 0.944 & 0.667 & 0.630 & 82.537 & 0.500 & 0.000 & 0.833 & 0.000 & 0.556 \\
\hline 88.253 & 0.625 & 0.500 & 0.750 & 0.667 & 0.600 & 86.636 & 0.633 & 0.167 & 0.944 & 0.667 & 0.630 & 87.445 & 0.500 & 0.000 & 0.833 & 0.000 & 0.556 \\
\hline 93.500 & 0.583 & 0.500 & 0.667 & 0.600 & 0.571 & 91.788 & 0.633 & 0.167 & 0.944 & 0.667 & 0.630 & 92.644 & 0.533 & 0.000 & 0.889 & 0.000 & 0.571 \\
\hline 99.060 & 0.625 & 0.500 & 0.750 & 0.667 & 0.600 & 97.246 & 0.633 & 0.167 & 0.944 & 0.667 & 0.630 & 98.153 & 0.567 & 0.083 & 0.889 & 0.333 & 0.593 \\
\hline 104.951 & 0.417 & 0.167 & 0.667 & 0.333 & 0.444 & 103.029 & 0.667 & 0.250 & 0.944 & 0.750 & 0.654 & 103.990 & 0.633 & 0.083 & 1.000 & 1.000 & 0.621 \\
\hline 111.191 & 0.375 & 0.083 & 0.667 & 0.200 & 0.421 & 109.155 & 0.633 & 0.167 & 0.944 & 0.667 & 0.630 & 110.173 & 0.667 & 0.167 & 1.000 & 1.000 & 0.643 \\
\hline 117.803 & 0.458 & 0.250 & 0.667 & 0.429 & 0.471 & 115.646 & 0.700 & 0.333 & 0.944 & 0.800 & 0.680 & 116.724 & 0.667 & 0.167 & 1.000 & 1.000 & 0.643 \\
\hline 124.808 & 0.417 & 0.167 & 0.667 & 0.333 & 0.444 & 122.522 & 0.600 & 0.167 & 0.889 & 0.500 & 0.615 & 123.665 & 0.667 & 0.167 & 1.000 & 1.000 & 0.643 \\
\hline
\end{tabular}
classifiers were trained with in-phase times of coherence features from setup 2 (2 features); at all subscales (periods in s.). Acc. - accuracy; Sen. - sensitivity; Spe. - specificity;
PPV - positive predictive value; NPV - negative predictive value; LOOVC - leave-one-out cross validation; in bold - ranges of periods at which the classifiers performed the best (= results shown in Table 5.2). 
TABLE B.5: Classification performances at all subscales with SVM polynomial kernel (setup $2=2$ features).

\begin{tabular}{|c|c|c|c|c|c|c|c|c|c|c|c|c|c|c|c|c|c|}
\hline \multicolumn{18}{|c|}{ Features based on VENT-FPL, VENT-FPR } \\
\hline \multicolumn{6}{|c|}{ In-House LOOCV (24 folds) } & \multicolumn{6}{|c|}{ Leuven LOOCV (30 folds) } & \multicolumn{6}{|c|}{ In-House validated by Leuven } \\
\hline Periods & Acc. & Sen. & Spe. & PPV & NPV & Periods & Acc. & Sen. & Spe. & PPV & NPV & Periods & Acc. & Sen. & Spe. & PPV & NPV \\
\hline 4.132 & 0.750 & 0.667 & 0.833 & 0.800 & 0.714 & 4.057 & 0.500 & 0.417 & 0.556 & 0.385 & 0.588 & 4.094 & 0.567 & 0.250 & 0.778 & 0.429 & 0.609 \\
\hline 4.378 & 0.583 & 0.583 & 0.583 & 0.583 & 0.583 & 4.298 & 0.533 & 0.583 & 0.500 & 0.438 & 0.643 & 4.338 & 0.600 & 0.250 & 0.833 & 0.500 & 0.625 \\
\hline 4.638 & 0.542 & 0.583 & 0.500 & 0.539 & 0.546 & 4.553 & 0.500 & 0.333 & 0.611 & 0.364 & 0.579 & 4.596 & 0.567 & 0.583 & 0.556 & 0.467 & 0.667 \\
\hline 4.914 & 0.625 & 0.750 & 0.500 & 0.600 & 0.667 & 4.824 & 0.467 & 0.250 & 0.611 & 0.300 & 0.550 & 4.869 & 0.600 & 0.750 & 0.500 & 0.500 & 0.750 \\
\hline 5.206 & 0.583 & 0.667 & 0.500 & 0.571 & 0.600 & 5.111 & 0.500 & 0.583 & 0.444 & 0.412 & 0.615 & 5.159 & 0.567 & 0.833 & 0.389 & 0.476 & 0.778 \\
\hline 5.516 & 0.667 & 0.583 & 0.750 & 0.700 & 0.643 & 5.415 & 0.600 & 0.333 & 0.778 & 0.500 & 0.636 & 5.465 & 0.533 & 0.000 & 0.889 & 0.000 & 0.571 \\
\hline 5.844 & 0.458 & 0.417 & 0.500 & 0.455 & 0.462 & 5.737 & 0.700 & 0.417 & 0.889 & 0.714 & 0.696 & 5.790 & 0.433 & 0.667 & 0.278 & 0.381 & 0.556 \\
\hline 6.191 & 0.708 & 0.750 & 0.667 & 0.692 & 0.727 & 6.078 & 0.567 & 0.167 & 0.833 & 0.400 & 0.600 & 6.135 & 0.500 & 0.917 & 0.222 & 0.440 & 0.800 \\
\hline 6.559 & 0.542 & 0.667 & 0.417 & 0.533 & 0.556 & 6.439 & 0.500 & 0.333 & 0.611 & 0.364 & 0.579 & 6.499 & 0.533 & 0.500 & 0.556 & 0.429 & 0.625 \\
\hline 6.950 & 0.417 & 0.500 & 0.333 & 0.429 & 0.400 & 6.822 & 0.633 & 0.500 & 0.722 & 0.546 & 0.684 & 6.886 & 0.400 & 0.250 & 0.500 & 0.250 & 0.500 \\
\hline 7.363 & 0.292 & 0.417 & 0.167 & 0.333 & 0.222 & 7.228 & 0.600 & 0.500 & 0.667 & 0.500 & 0.667 & 7.295 & 0.400 & 0.500 & 0.333 & 0.333 & 0.500 \\
\hline 7.801 & 0.292 & 0.417 & 0.167 & 0.333 & 0.222 & 7.658 & 0.533 & 0.583 & 0.500 & 0.438 & 0.643 & 7.729 & 0.467 & 1.000 & 0.111 & 0.429 & 1.000 \\
\hline 8.264 & 0.208 & 0.250 & 0.167 & 0.231 & 0.182 & 8.113 & 0.333 & 0.250 & 0.389 & 0.214 & 0.438 & 8.189 & 0.467 & 0.500 & 0.444 & 0.375 & 0.571 \\
\hline 8.756 & 0.458 & 0.500 & 0.417 & 0.462 & 0.455 & 8.595 & 0.567 & 0.417 & 0.667 & 0.455 & 0.632 & 8.676 & 0.567 & 0.667 & 0.500 & 0.471 & 0.692 \\
\hline 9.276 & 0.792 & 0.833 & 0.750 & 0.769 & 0.818 & 9.107 & 0.533 & 0.500 & 0.556 & 0.429 & 0.625 & 9.192 & 0.700 & 0.667 & 0.722 & 0.615 & 0.765 \\
\hline 9.828 & 0.625 & 0.833 & 0.417 & 0.588 & 0.714 & 9.648 & 0.767 & 0.750 & 0.778 & 0.692 & 0.824 & 9.738 & 0.633 & 0.583 & 0.667 & 0.539 & 0.706 \\
\hline 10.412 & 0.375 & 0.333 & 0.417 & 0.364 & 0.385 & 10.222 & 0.833 & 0.917 & 0.778 & 0.733 & 0.933 & 10.317 & 0.567 & 0.833 & 0.389 & 0.476 & 0.778 \\
\hline 11.032 & 0.458 & 0.417 & 0.500 & 0.455 & 0.462 & 10.830 & 0.767 & 0.833 & 0.722 & 0.667 & 0.867 & 10.931 & 0.800 & 1.000 & 0.667 & 0.667 & 1.000 \\
\hline 11.688 & 0.333 & 0.333 & 0.333 & 0.333 & 0.333 & 11.474 & 0.533 & 0.500 & 0.556 & 0.429 & 0.625 & 11.581 & 0.600 & 0.500 & 0.667 & 0.500 & 0.667 \\
\hline 12.383 & 0.542 & 0.583 & 0.500 & 0.539 & 0.546 & 12.156 & 0.633 & 0.667 & 0.611 & 0.533 & 0.733 & 12.269 & 0.467 & 0.333 & 0.556 & 0.333 & 0.556 \\
\hline 13.119 & 0.458 & 0.583 & 0.333 & 0.467 & 0.444 & 12.879 & 0.467 & 0.583 & 0.389 & 0.389 & 0.583 & 12.999 & 0.667 & 0.917 & 0.500 & 0.550 & 0.900 \\
\hline 13.899 & 0.542 & 0.500 & 0.583 & 0.546 & 0.539 & 13.644 & 0.567 & 0.500 & 0.611 & 0.462 & 0.647 & 13.772 & 0.533 & 0.917 & 0.278 & 0.458 & 0.833 \\
\hline 14.725 & 0.458 & 0.500 & 0.417 & 0.462 & 0.455 & 14.456 & 0.633 & 0.583 & 0.667 & 0.539 & 0.706 & 14.591 & 0.533 & 0.750 & 0.389 & 0.450 & 0.700 \\
\hline 15.601 & 0.458 & 0.583 & 0.333 & 0.467 & 0.444 & 15.315 & 0.600 & 0.667 & 0.556 & 0.500 & 0.714 & 15.458 & 0.367 & 0.333 & 0.389 & 0.267 & 0.467 \\
\hline 16.529 & 0.542 & 0.667 & 0.417 & 0.533 & 0.556 & 16.226 & 0.533 & 0.583 & 0.500 & 0.438 & 0.643 & 16.377 & 0.333 & 0.500 & 0.222 & 0.300 & 0.400 \\
\hline 17.512 & 0.375 & 0.333 & 0.417 & 0.364 & 0.385 & 17.191 & 0.600 & 0.417 & 0.722 & 0.500 & 0.650 & 17.351 & 0.433 & 0.917 & 0.111 & 0.407 & 0.667 \\
\hline 18.553 & 0.333 & 0.417 & 0.250 & 0.357 & 0.300 & 18.213 & 0.667 & 0.500 & 0.778 & 0.600 & 0.700 & 18.383 & 0.567 & 0.917 & 0.333 & 0.478 & 0.857 \\
\hline 19.656 & 0.292 & 0.333 & 0.250 & 0.308 & 0.273 & 19.296 & 0.567 & 0.583 & 0.556 & 0.467 & 0.667 & 19.476 & 0.433 & 0.500 & 0.389 & 0.353 & 0.539 \\
\hline 20.825 & 0.458 & 0.417 & 0.500 & 0.455 & 0.462 & 20.443 & 0.567 & 0.500 & 0.611 & 0.462 & 0.647 & 20.634 & 0.433 & 0.333 & 0.500 & 0.308 & 0.529 \\
\hline 22.063 & 0.500 & 0.583 & 0.417 & 0.500 & 0.500 & 21.659 & 0.633 & 0.750 & 0.556 & 0.529 & 0.769 & 21.861 & 0.600 & 0.750 & 0.500 & 0.500 & 0.750 \\
\hline 23.375 & 0.667 & 0.750 & 0.583 & 0.643 & 0.700 & 22.947 & 0.667 & 0.667 & 0.667 & 0.571 & 0.750 & 23.161 & 0.600 & 0.750 & 0.500 & 0.500 & 0.750 \\
\hline 24.765 & 0.750 & 0.750 & 0.750 & 0.750 & 0.750 & 24.312 & 0.333 & 0.167 & 0.444 & 0.167 & 0.444 & 24.538 & 0.600 & 0.750 & 0.500 & 0.500 & 0.750 \\
\hline 26.238 & 0.417 & 0.500 & 0.333 & 0.429 & 0.400 & 25.757 & 0.433 & 0.250 & 0.556 & 0.273 & 0.526 & 25.997 & 0.467 & 0.417 & 0.500 & 0.357 & 0.563 \\
\hline 27.798 & 0.750 & 0.917 & 0.583 & 0.688 & 0.875 & 27.289 & 0.567 & 0.417 & 0.667 & 0.455 & 0.632 & 27.543 & 0.300 & 0.417 & 0.222 & 0.263 & 0.364 \\
\hline 29.451 & 0.667 & 0.833 & 0.500 & 0.625 & 0.750 & 28.911 & 0.700 & 0.500 & 0.833 & 0.667 & 0.714 & 29.181 & 0.367 & 0.417 & 0.333 & 0.294 & 0.462 \\
\hline 31.202 & 0.667 & 0.750 & 0.583 & 0.643 & 0.700 & 30.631 & 0.633 & 0.333 & 0.833 & 0.571 & 0.652 & 30.916 & 0.633 & 0.417 & 0.778 & 0.556 & 0.667 \\
\hline 33.057 & 0.500 & 0.583 & 0.417 & 0.500 & 0.500 & 32.452 & 0.667 & 0.417 & 0.833 & 0.625 & 0.682 & 32.755 & 0.600 & 0.333 & 0.778 & 0.500 & 0.636 \\
\hline 35.023 & 0.500 & 0.417 & 0.583 & 0.500 & 0.500 & 34.382 & 0.600 & 0.417 & 0.722 & 0.500 & 0.650 & 34.702 & 0.600 & 0.167 & 0.889 & 0.500 & 0.615 \\
\hline 37.106 & 0.458 & 0.417 & 0.500 & 0.455 & 0.462 & 36.426 & 0.533 & 0.250 & 0.722 & 0.375 & 0.591 & 36.766 & 0.600 & 0.083 & 0.944 & 0.500 & 0.607 \\
\hline 39.312 & 0.542 & 0.500 & 0.583 & 0.546 & 0.539 & 38.592 & 0.633 & 0.333 & 0.833 & 0.571 & 0.652 & 38.952 & 0.533 & 0.083 & 0.833 & 0.250 & 0.577 \\
\hline 41.650 & 0.542 & 0.500 & 0.583 & 0.546 & 0.539 & 40.887 & 0.467 & 0.083 & 0.722 & 0.167 & 0.542 & 41.268 & 0.400 & 0.833 & 0.111 & 0.385 & 0.500 \\
\hline 44.126 & 0.542 & 0.333 & 0.750 & 0.571 & 0.529 & 43.318 & 0.633 & 0.333 & 0.833 & 0.571 & 0.652 & 43.722 & 0.533 & 0.167 & 0.778 & 0.333 & 0.583 \\
\hline 46.750 & 0.542 & 0.333 & 0.750 & 0.571 & 0.529 & 45.894 & 0.467 & 0.083 & 0.722 & 0.167 & 0.542 & 46.322 & 0.400 & 0.000 & 0.667 & 0.000 & 0.500 \\
\hline 49.530 & 0.583 & 0.417 & 0.750 & 0.625 & 0.563 & 48.623 & 0.433 & 0.000 & 0.722 & 0.000 & 0.520 & 49.077 & 0.500 & 0.083 & 0.778 & 0.200 & 0.560 \\
\hline 52.475 & 0.417 & 0.333 & 0.500 & 0.400 & 0.429 & 51.514 & 0.533 & 0.167 & 0.778 & 0.333 & 0.583 & 51.995 & 0.467 & 0.167 & 0.667 & 0.250 & 0.546 \\
\hline 55.596 & 0.458 & 0.167 & 0.750 & 0.400 & 0.474 & 54.577 & 0.600 & 0.000 & 1.000 & $\mathrm{NaN}$ & 0.600 & 55.087 & 0.400 & 0.167 & 0.556 & 0.200 & 0.500 \\
\hline 58.902 & 0.500 & 0.250 & 0.750 & 0.500 & 0.500 & 57.823 & 0.333 & 0.000 & 0.556 & 0.000 & 0.455 & 58.362 & 0.367 & 0.250 & 0.444 & 0.231 & 0.471 \\
\hline 62.404 & 0.542 & 0.250 & 0.833 & 0.600 & 0.526 & 61.261 & 0.500 & 0.750 & 0.333 & 0.429 & 0.667 & 61.833 & 0.467 & 0.167 & 0.667 & 0.250 & 0.546 \\
\hline 66.115 & 0.458 & 0.250 & 0.667 & 0.429 & 0.471 & 64.904 & 0.567 & 0.000 & 0.944 & 0.000 & 0.586 & 65.509 & 0.533 & 0.167 & 0.778 & 0.333 & 0.583 \\
\hline 70.046 & 0.500 & 0.167 & 0.833 & 0.500 & 0.500 & 68.763 & 0.600 & 0.083 & 0.944 & 0.500 & 0.607 & 69.405 & 0.533 & 0.083 & 0.833 & 0.250 & 0.577 \\
\hline 74.211 & 0.208 & 0.167 & 0.250 & 0.182 & 0.231 & 72.852 & 0.600 & 0.083 & 0.944 & 0.500 & 0.607 & 73.532 & 0.533 & 0.083 & 0.833 & 0.250 & 0.577 \\
\hline 78.624 & 0.208 & 0.083 & 0.333 & 0.111 & 0.267 & 77.184 & 0.600 & 0.083 & 0.944 & 0.500 & 0.607 & 77.904 & 0.400 & 0.917 & 0.056 & 0.393 & 0.500 \\
\hline 83.299 & 0.542 & 0.417 & 0.667 & 0.556 & 0.533 & 81.774 & 0.633 & 0.167 & 0.944 & 0.667 & 0.630 & 82.537 & 0.633 & 0.083 & 1.000 & 1.000 & 0.621 \\
\hline 88.253 & 0.583 & 0.500 & 0.667 & 0.600 & 0.571 & 86.636 & 0.567 & 0.083 & 0.889 & 0.333 & 0.593 & 87.445 & 0.500 & 0.083 & 0.778 & 0.200 & 0.560 \\
\hline 93.500 & 0.667 & 0.500 & 0.833 & 0.750 & 0.625 & 91.788 & 0.600 & 0.083 & 0.944 & 0.500 & 0.607 & 92.644 & 0.533 & 0.000 & 0.889 & 0.000 & 0.571 \\
\hline 99.060 & 0.458 & 0.333 & 0.583 & 0.444 & 0.467 & 97.246 & 0.600 & 0.083 & 0.944 & 0.500 & 0.607 & 98.153 & 0.633 & 0.250 & 0.889 & 0.600 & 0.640 \\
\hline 104.951 & 0.542 & 0.333 & 0.750 & 0.571 & 0.529 & 103.029 & 0.667 & 0.250 & 0.944 & 0.750 & 0.654 & 103.990 & 0.733 & 0.333 & 1.000 & 1.000 & 0.692 \\
\hline 111.191 & 0.500 & 0.250 & 0.750 & 0.500 & 0.500 & 109.155 & 0.600 & 0.083 & 0.944 & 0.500 & 0.607 & 110.173 & 0.667 & 0.250 & 0.944 & 0.750 & 0.654 \\
\hline 117.803 & 0.542 & 0.250 & 0.833 & 0.600 & 0.526 & 115.646 & 0.633 & 0.250 & 0.889 & 0.600 & 0.640 & 116.724 & 0.733 & 0.500 & 0.889 & 0.750 & 0.727 \\
\hline 124.808 & 0.417 & 0.167 & 0.667 & 0.333 & 0.444 & 122.522 & 0.600 & 0.250 & 0.833 & 0.500 & 0.625 & 123.665 & 0.633 & 0.167 & 0.944 & 0.667 & 0.630 \\
\hline
\end{tabular}

classifiers were trained with in-phase times of coherence features from setup 2 (2 features); at all subscales (periods in s.). Acc. - accuracy; Sen. - sensitivity; Spe. - specificity;
PPV - positive predictive value; NPV - negative predictive value; LOOVC - leave-one-out cross validation; in bold - ranges of periods at which the classifiers performed the best (= results shown in Table 5.2). 
TABLE B.6: Classification performances at all subscales with SVM rbf kernel (setup $2=2$ features).

\begin{tabular}{|c|c|c|c|c|c|c|c|c|c|c|c|c|c|c|c|c|c|}
\hline \multicolumn{18}{|c|}{ Features based on VENT-FPL, VENT-FPR } \\
\hline \multicolumn{6}{|c|}{ In-House LOOCV ( 24 folds) } & \multicolumn{6}{|c|}{ Leuven LOOCV (30 folds) } & \multicolumn{6}{|c|}{ In-House validated by Leuven } \\
\hline Periods & Acc. & Sen. & Spe. & PPV & NPV & Periods & Acc. & Sens. & Spe. & PPV & NPV & Periods & Acc. & Sen. & Spe. & PPV & NPV \\
\hline 4.132 & 0.667 & 0.500 & 0.833 & 0.750 & 0.625 & 4.057 & 0.533 & 0.000 & 0.889 & 0.000 & 0.571 & 4.094 & 0.600 & 0.000 & 1.000 & $\mathrm{NaN}$ & 0.600 \\
\hline 4.378 & 0.667 & 0.667 & 0.667 & 0.667 & 0.667 & 4.298 & 0.600 & 0.333 & 0.778 & 0.500 & 0.636 & 4.338 & 0.600 & 0.083 & 0.944 & 0.500 & 0.607 \\
\hline 4.638 & 0.625 & 0.833 & 0.417 & 0.588 & 0.714 & 4.553 & 0.500 & 0.000 & 0.833 & 0.000 & 0.556 & 4.596 & 0.567 & 0.083 & 0.889 & 0.333 & 0.593 \\
\hline 4.914 & 0.583 & 0.750 & 0.417 & 0.563 & 0.625 & 4.824 & 0.400 & 0.000 & 0.667 & 0.000 & 0.500 & 4.869 & 0.533 & 0.417 & 0.611 & 0.417 & 0.611 \\
\hline 5.206 & 0.708 & 0.583 & 0.833 & 0.778 & 0.667 & 5.111 & 0.533 & 0.250 & 0.722 & 0.375 & 0.591 & 5.159 & 0.567 & 0.333 & 0.722 & 0.444 & 0.619 \\
\hline 5.516 & 0.792 & 0.750 & 0.833 & 0.818 & 0.769 & 5.415 & 0.567 & 0.250 & 0.778 & 0.429 & 0.609 & 5.465 & 0.467 & 0.250 & 0.611 & 0.300 & 0.550 \\
\hline 5.844 & 0.292 & 0.250 & 0.333 & 0.273 & 0.308 & 5.737 & 0.533 & 0.167 & 0.778 & 0.333 & 0.583 & 5.790 & 0.500 & 0.833 & 0.278 & 0.435 & 0.714 \\
\hline 6.191 & 0.542 & 0.500 & 0.583 & 0.546 & 0.539 & 6.078 & 0.500 & 0.167 & 0.722 & 0.286 & 0.565 & 6.135 & 0.467 & 0.833 & 0.222 & 0.417 & 0.667 \\
\hline 6.559 & 0.500 & 0.500 & 0.500 & 0.500 & 0.500 & 6.439 & 0.533 & 0.167 & 0.778 & 0.333 & 0.583 & 6.499 & 0.533 & 0.833 & 0.333 & 0.455 & 0.750 \\
\hline 6.950 & 0.458 & 0.333 & 0.583 & 0.444 & 0.467 & 6.822 & 0.600 & 0.333 & 0.778 & 0.500 & 0.636 & 6.886 & 0.533 & 0.667 & 0.444 & 0.444 & 0.667 \\
\hline 7.363 & 0.417 & 0.417 & 0.417 & 0.417 & 0.417 & 7.228 & 0.600 & 0.250 & 0.833 & 0.500 & 0.625 & 7.295 & 0.467 & 0.417 & 0.500 & 0.357 & 0.563 \\
\hline 7.801 & 0.542 & 0.500 & 0.583 & 0.546 & 0.539 & 7.658 & 0.500 & 0.167 & 0.722 & 0.286 & 0.565 & 7.729 & 0.467 & 0.417 & 0.500 & 0.357 & 0.563 \\
\hline 8.264 & 0.333 & 0.333 & 0.333 & 0.333 & 0.333 & 8.113 & 0.467 & 0.167 & 0.667 & 0.250 & 0.546 & 8.189 & 0.433 & 0.667 & 0.278 & 0.381 & 0.556 \\
\hline 8.756 & 0.542 & 0.500 & 0.583 & 0.546 & 0.539 & 8.595 & 0.633 & 0.417 & 0.778 & 0.556 & 0.667 & 8.676 & 0.600 & 0.667 & 0.556 & 0.500 & 0.714 \\
\hline 9.276 & 0.500 & 0.583 & 0.417 & 0.500 & 0.500 & 9.107 & 0.667 & 0.583 & 0.722 & 0.583 & 0.722 & 9.192 & 0.600 & 0.833 & 0.444 & 0.500 & 0.800 \\
\hline 9.828 & 0.625 & 0.667 & 0.583 & 0.615 & 0.636 & 9.648 & 0.800 & 0.750 & 0.833 & 0.750 & 0.833 & 9.738 & 0.600 & 0.583 & 0.611 & 0.500 & 0.688 \\
\hline 10.412 & 0.458 & 0.250 & 0.667 & 0.429 & 0.471 & 10.222 & 0.867 & 0.917 & 0.833 & 0.786 & 0.938 & 10.317 & 0.433 & 0.500 & 0.389 & 0.353 & 0.539 \\
\hline 11.032 & 0.500 & 0.417 & 0.583 & 0.500 & 0.500 & 10.830 & 0.733 & 0.667 & 0.778 & 0.667 & 0.778 & 10.931 & 0.567 & 0.333 & 0.722 & 0.444 & 0.619 \\
\hline 11.688 & 0.458 & 0.333 & 0.583 & 0.444 & 0.467 & 11.474 & 0.600 & 0.417 & 0.722 & 0.500 & 0.650 & 11.581 & 0.633 & 0.750 & 0.556 & 0.529 & 0.769 \\
\hline 12.383 & 0.500 & 0.500 & 0.500 & 0.500 & 0.500 & 12.156 & 0.733 & 0.667 & 0.778 & 0.667 & 0.778 & 12.269 & 0.567 & 0.333 & 0.722 & 0.444 & 0.619 \\
\hline 13.119 & 0.458 & 0.583 & 0.333 & 0.467 & 0.444 & 12.879 & 0.500 & 0.250 & 0.667 & 0.333 & 0.571 & 12.999 & 0.667 & 0.667 & 0.667 & 0.571 & 0.750 \\
\hline 13.899 & 0.500 & 0.667 & 0.333 & 0.500 & 0.500 & 13.644 & 0.733 & 0.583 & 0.833 & 0.700 & 0.750 & 13.772 & 0.500 & 0.917 & 0.222 & 0.440 & 0.800 \\
\hline 14.725 & 0.292 & 0.333 & 0.250 & 0.308 & 0.273 & 14.456 & 0.600 & 0.333 & 0.778 & 0.500 & 0.636 & 14.591 & 0.533 & 0.917 & 0.278 & 0.458 & 0.833 \\
\hline 15.601 & 0.375 & 0.500 & 0.250 & 0.400 & 0.333 & 15.315 & 0.533 & 0.250 & 0.722 & 0.375 & 0.591 & 15.458 & 0.500 & 1.000 & 0.167 & 0.444 & 1.000 \\
\hline 16.529 & 0.333 & 0.250 & 0.417 & 0.300 & 0.357 & 16.226 & 0.700 & 0.500 & 0.833 & 0.667 & 0.714 & 77 & 0.433 & 0.750 & 0.222 & 0.391 & 0.571 \\
\hline 17.512 & 0.417 & 0.500 & 0.333 & 0.429 & 0.400 & 17.191 & 0.567 & 0.333 & 0.722 & 0.444 & 0.619 & 17.351 & 0.567 & 0.917 & 0.333 & 0.478 & 0.857 \\
\hline 18.553 & 0.000 & 0.000 & 0.000 & 0.000 & 0.000 & 18.213 & 0.633 & 0.417 & 0.778 & 0.556 & 0.667 & 18.383 & 0.567 & 0.833 & 0.389 & 0.476 & 0.778 \\
\hline 19.656 & 0.458 & 0.417 & 0.500 & 0.455 & 0.462 & 19.296 & 0.633 & 0.500 & 0.722 & 0.546 & 0.684 & 19.476 & 0.567 & 0.500 & 0.611 & 0.462 & 0.647 \\
\hline 20.825 & 0.333 & 0.417 & 0.250 & 0.357 & 0.300 & 20.443 & 0.567 & 0.417 & 0.667 & 0.455 & 0.632 & 20.634 & 0.533 & 0.667 & 0.444 & 0.444 & 0.667 \\
\hline 22.063 & 0.542 & 0.417 & 0.667 & 0.556 & 0.533 & 21.659 & 0.600 & 0.500 & 0.667 & 0.500 & 0.667 & 21.861 & 0.700 & 0.583 & 0.778 & 0.636 & 0.737 \\
\hline 23.375 & 0.542 & 0.417 & 0.667 & 0.556 & 0.533 & 22.947 & 0.700 & 0.500 & 0.833 & 0.667 & 0.714 & 23.161 & 0.633 & 0.500 & 0.722 & 0.546 & 0.684 \\
\hline 24.765 & 0.708 & 0.750 & 0.667 & 0.692 & 0.727 & 24.312 & 0.567 & 0.000 & 0.944 & 0.000 & 0.586 & 24.538 & 0.533 & 0.500 & 0.556 & 0.429 & 0.625 \\
\hline 26.238 & 0.167 & 0.333 & 0.000 & 0.250 & 0.000 & 25.757 & 0.500 & 0.167 & 0.722 & 0.286 & 0.565 & 25.997 & 0.500 & 0.500 & 0.500 & 0.400 & 0.600 \\
\hline 27.798 & 0.333 & 0.500 & 0.167 & 0.375 & 0.250 & 27.289 & 0.600 & 0.417 & 0.722 & 0.500 & 0.650 & 27.543 & 0.367 & 0.500 & 0.278 & 0.316 & 0.455 \\
\hline 29.451 & 0.792 & 0.917 & 0.667 & 0.733 & 0.889 & 28.911 & 0.533 & 0.250 & 0.722 & 0.375 & 0.591 & 29.181 & 0.400 & 0.667 & 0.222 & 0.364 & 0.500 \\
\hline 31.202 & 0.625 & 0.417 & 0.833 & 0.714 & 0.588 & 30631 & 0.533 & 0.000 & 0.889 & 0.000 & 0.571 & 30.916 & 0.633 & 0.167 & 0.944 & 0.667 & 0.630 \\
\hline 33.057 & 0.458 & 0.583 & 0.333 & 0.467 & 0.444 & 32.452 & 0.567 & 0.167 & 0.833 & 0.400 & 0.600 & 32.755 & 0.533 & 0.333 & 0.667 & 0.400 & 0.600 \\
\hline 35.023 & 0542 & 0.500 & 0.583 & 0.546 & 0.539 & 34382 & 0.600 & 0.333 & 0.778 & 0.500 & 0.636 & & 0.567 & 0.167 & 0.833 & 0.400 & 0.600 \\
\hline 37.106 & 0.625 & 0.583 & 0.667 & 0.636 & 0.615 & 36.426 & 0.667 & 0.250 & 0.944 & 0.750 & 0.654 & 36.766 & 0.567 & 0.083 & 0.889 & 0.333 & 0.593 \\
\hline 39.312 & 0.500 & 0.667 & 0.333 & 0.500 & 0.500 & 2 & 0.600 & 0.0 & 0.944 & 0.500 & 0.607 & & 0.267 & 0.667 & 0.000 & 0.308 & 0.000 \\
\hline 41.650 & 0.583 & 0.667 & 0.500 & 0.571 & 0.600 & 4 & 0.533 & 0.0 & 0.889 & 0.000 & 0.571 & 41. & 0.433 & 0.917 & 0.111 & 0.407 & 0.667 \\
\hline 44.126 & 0.583 & 0.667 & 0.500 & 0.571 & 0.600 & 43.318 & 0.567 & 0.000 & 0.944 & 0.000 & 0.586 & 43.722 & 0.467 & 0.167 & 0.667 & 0.250 & 0.546 \\
\hline 46.750 & 0.667 & 0.583 & 0.750 & 0.700 & 0.643 & 45.894 & 0.567 & 0.000 & 0.944 & 0.000 & 0.586 & 46.322 & 0.500 & 0.250 & 0.667 & 0.333 & 0.571 \\
\hline 49.530 & 0.500 & 0.500 & 0.500 & 0.500 & 0.500 & 48.623 & 0.533 & 0.000 & 0.889 & 0.000 & 0.571 & 49.077 & 0.533 & 0.250 & 0.722 & 0.375 & 0.591 \\
\hline 52.475 & 0.542 & 0.417 & 0.667 & 0.55 & 0.533 & 51514 & 0.600 & & 1.000 & $\mathrm{NaN}$ & & & & 0.167 & 0.667 & 0.250 & 0.546 \\
\hline 55.596 & 0.542 & 0.583 & 0.500 & 0.539 & 0.546 & 54.577 & 0.600 & 0.000 & 1.000 & $\mathrm{NaN}$ & 0.600 & 55.087 & 0.400 & 0.250 & 0.500 & 0.250 & 0.500 \\
\hline 58.902 & 0.542 & 0.250 & 0.833 & 0.600 & 0.526 & 57892 & 0.600 & 0.000 & 1.000 & $\mathrm{NaN}$ & 0.600 & 58362 & 0.467 & 0.250 & 0.611 & 0.300 & 0.550 \\
\hline 62.404 & 0.250 & 0.000 & 0.500 & & & 61.261 & 0.300 & 0. & 0.500 & 0.000 & 0.429 & 3 & 0.433 & 0.250 & 0.556 & 0.273 & 0.526 \\
\hline 66.115 & 0.500 & 0.333 & 0.667 & 0.500 & 0.500 & 64904 & 0.600 & 0.000 & 1.000 & $\mathrm{NaN}$ & 0.600 & 65.509 & 0.533 & 0.083 & 0.833 & 0.250 & 0.577 \\
\hline 70.046 & 0.4 & & 0.750 & & & & & & 1.000 & $\mathrm{NaN}$ & & 69.405 & & 0.083 & 0.833 & 0.250 & 0.577 \\
\hline 74.211 & 0.375 & 0.167 & 0.583 & 0.286 & 0.412 & 72.852 & 0.567 & 0.000 & 0.944 & 0.000 & 0.586 & 73.532 & 0.533 & 0.083 & 0.833 & 0.250 & 0.577 \\
\hline 78.624 & 0.333 & 0.333 & 0.33 & 0.33 & 0.333 & & 0.567 & 0.000 & 0.944 & 0.000 & 0.586 & 77.904 & 0.533 & 0.083 & 0.833 & 0.250 & 0.577 \\
\hline 83.299 & 0.292 & 0.167 & 0.417 & 0.222 & 0.333 & 81.774 & 0.600 & 0.083 & 0.944 & 0.500 & 0.607 & 82.537 & 0.600 & 0.000 & 1.000 & $\mathrm{NaN}$ & 0.600 \\
\hline 88.253 & 0.625 & 0.5 & 0.7 & 0.6 & 0.6 & & 0.6 & 0.0 & 1.000 & $\mathrm{NaN}$ & 0.600 & 87.445 & 0.500 & 0.000 & 0.833 & 0.000 & 0.556 \\
\hline 93.500 & 0.708 & 0.500 & 0.917 & 0.857 & 0.647 & 91.788 & 0.567 & 0.000 & 0.944 & 0.000 & 0.586 & 92.644 & 0.533 & 0.000 & 0.889 & 0.000 & 0.571 \\
\hline 99.060 & 0.458 & 0.333 & 0.583 & 0.444 & 0.467 & 97.246 & 0.533 & 0.000 & 0.889 & 0.000 & 0.571 & 98.153 & 0.600 & 0.167 & 0.889 & 0.500 & 0.615 \\
\hline 104.951 & 0.500 & 0.333 & 0.667 & 0.500 & 0.500 & 103.029 & 0.667 & 0.250 & 0.944 & 0.750 & 0.654 & 103.990 & 0.667 & 0.167 & 1.000 & 1.000 & 0.643 \\
\hline 111.191 & 0.500 & 0.333 & 0.667 & 0.500 & 0.500 & 109.155 & 0.633 & 0.167 & 0.944 & 0.667 & 0.630 & 110.173 & 0.700 & 0.333 & 0.944 & 0.800 & 0.680 \\
\hline 117.803 & 0.458 & 0.250 & 0.667 & 0.4 & 0.471 & 115.646 & 0.700 & 0.4 & 0.889 & 0.714 & 0.696 & & 0.733 & 0.500 & 0.889 & 0.750 & 0.727 \\
\hline 124.808 & 0.417 & 0.167 & 0.667 & 0.333 & 0.444 & 122.522 & 0.667 & 0.333 & 0.889 & 0.667 & 0.667 & 123.665 & 0.700 & 0.500 & 0.833 & 0.667 & 0.714 \\
\hline
\end{tabular}

PPV - positive predictive value; NPV - negative predictive value; LOOVC - leave-one-out cross validation; grey highlights - accuracies; in bold - ranges of periods at which the

classifiers performed the best (= results shown in Table 5.2). 


\section{Appendix C}

\section{Appendix Chapter 6}

\section{C.1 Figures of the best feature for each dynamics metric}

Figures C.1-C.4 show the best feature selection for each of the dynamics metric after 5000-permutation testing thresholded at (uncorrected) $\mathrm{p}<0.05$.

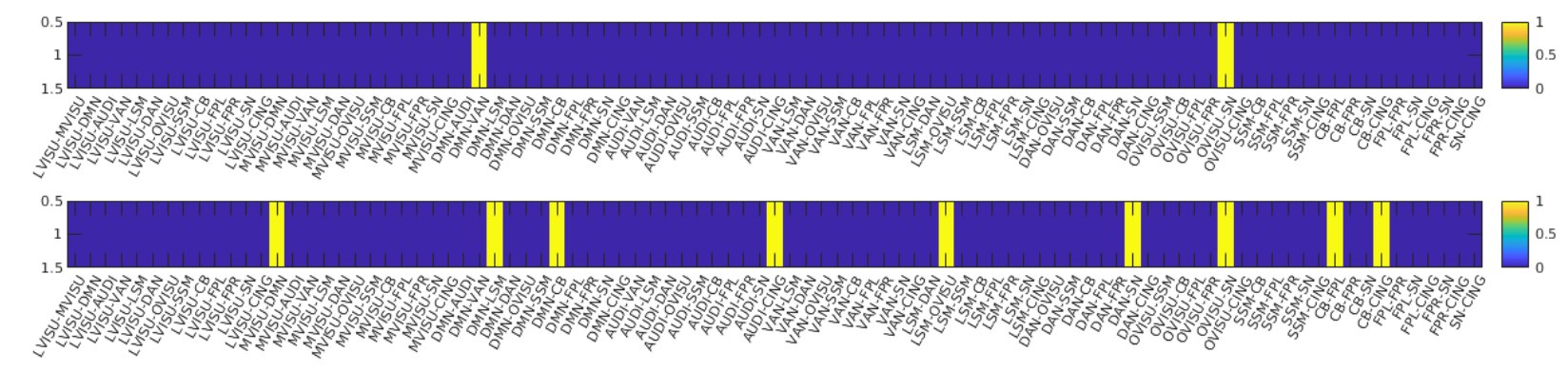

FIGURE C.1: Static functional connectivity best feature selection. Yellow - significant $(\mathrm{p}<0.05)$; dark blue non-significant. 


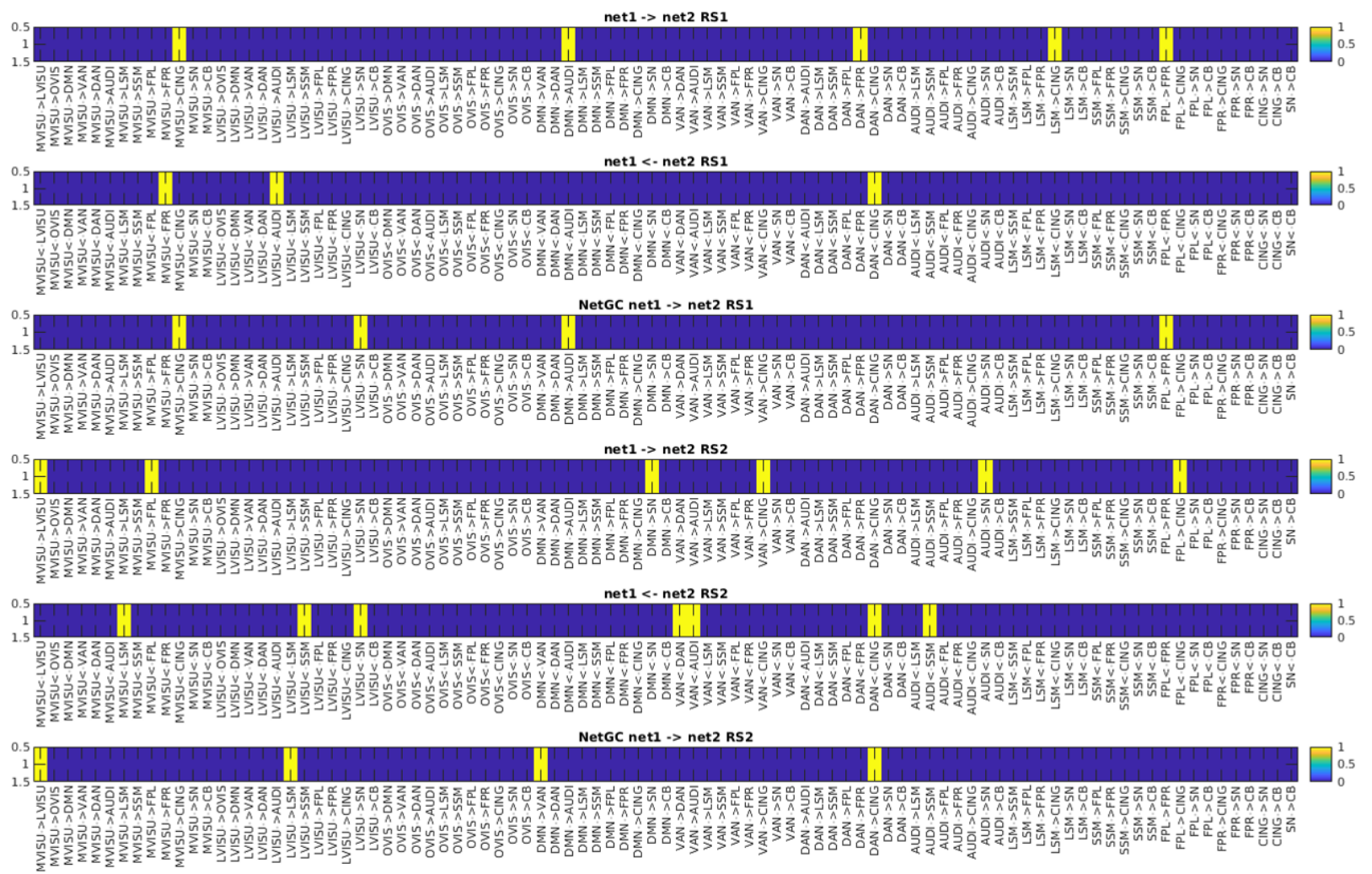

FIGURE C.2: Pairwise Granger causality best feature selection. Yellow - significant $(p<0.05)$; dark blue non-significant.
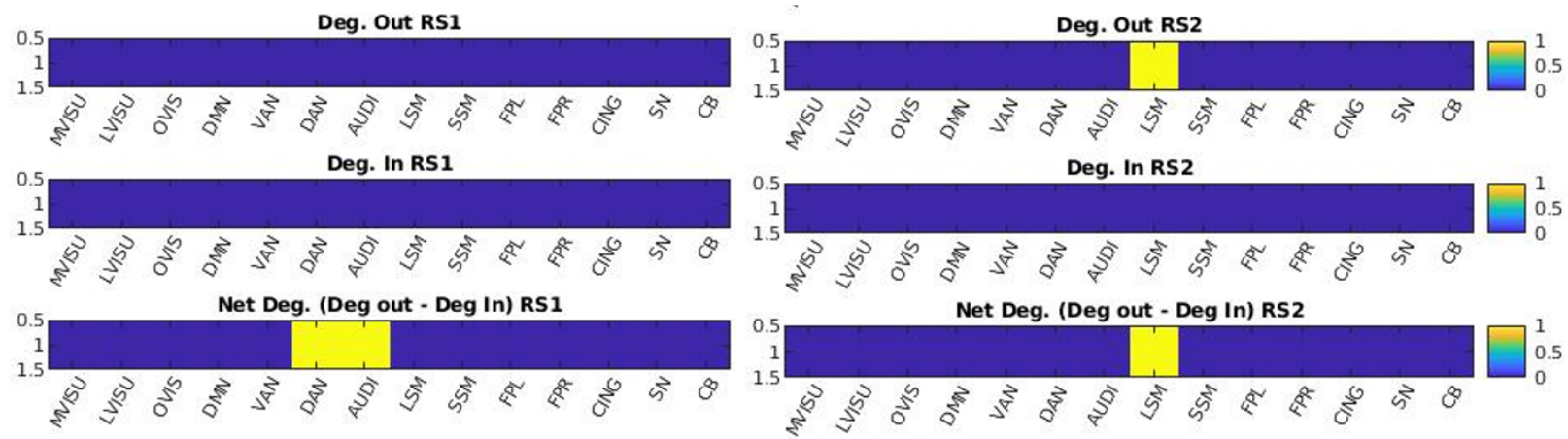

FIGURE C.3: Granger causality degree best feature selection. Yellow - significant $(\mathrm{p}<0.05)$; dark blue non-significant.
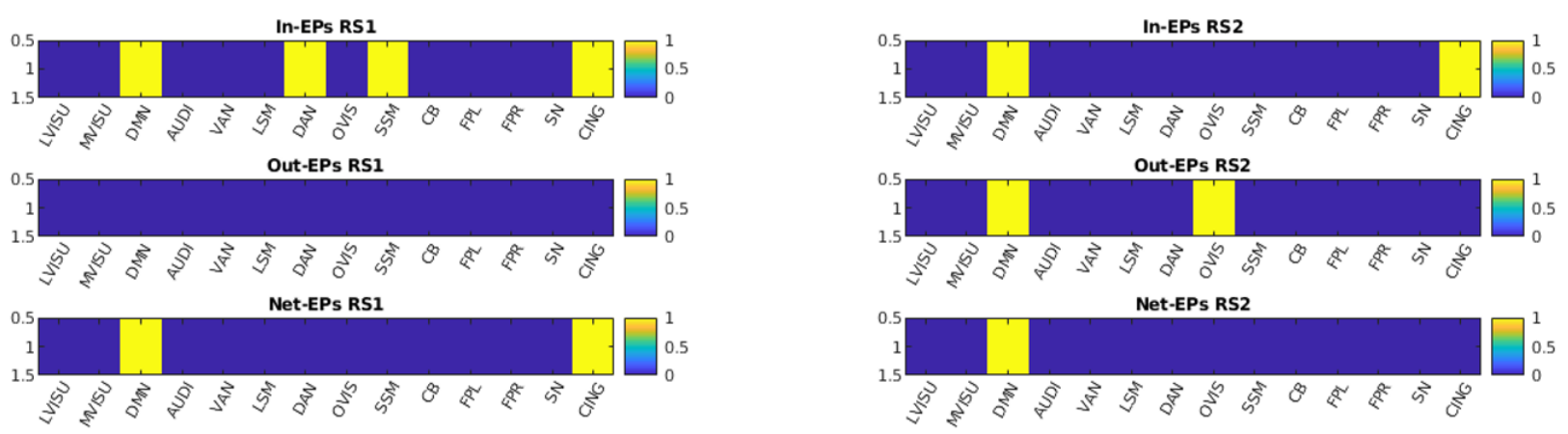

FIGURE C.4: Emulative power best feature selection. Yellow - significant $(\mathrm{p}<0.05)$; dark blue - nonsignificant. 


\section{C.2 Feature setups giving the best classification performances}

TABLE C.1: Classifier setups used in Tables 6.3 and 6.4 (Chapter 6).

\begin{tabular}{|c|c|c|c|c|c|c|c|c|}
\hline \multirow{2}{*}{$\frac{\text { Setup \# }}{1}$} & \multicolumn{2}{|c|}{ static FC } & \multicolumn{2}{|c|}{ Granger causality } & \multicolumn{2}{|c|}{ Wavelet Coherence } & \multicolumn{2}{|c|}{ Evolutionay game theory } \\
\hline & - & & - & & - & & $\begin{array}{l}\text { InEp DMN } \\
\text { OutEP DMN } \\
\text { NetEP DMN }\end{array}$ & $\begin{array}{l}\text { InEP DAN } \\
\text { OutEP DAN } \\
\text { NetEP DAN }\end{array}$ \\
\hline 2 & - & & - & & ip OVIS-SN s2 & ip MVISU-DMN s2 & - & \\
\hline 3 & $\begin{array}{l}\text { sFC DMN-VAN } \\
\text { sFC MVISU-DMN } \\
\text { sFC DMN-LSM }\end{array}$ & $\begin{array}{l}\text { sFC DMN-CB } \\
\text { sFC DAN-SN } \\
\text { sFC OVIS-SN }\end{array}$ & $\begin{array}{l}- \\
- \\
-\end{array}$ & & $\begin{array}{l}- \\
- \\
-\end{array}$ & & $\begin{array}{l}- \\
- \\
-\end{array}$ & \\
\hline 4 & $\begin{array}{l}- \\
- \\
- \\
- \\
- \\
- \\
-\end{array}$ & & $\begin{array}{l}\text { DMN } \rightarrow \text { AUDI } \\
\text { MVISU } \leftarrow \text { AUDI } \\
\text { LVISU } \leftarrow \text { OVIS } \\
\text { OVISUAUDI } \\
\text { FPR } \leftarrow \text { CB } \\
\text { netgc MVISU } \rightarrow \text { CING } \\
\text { netgc DMN } \rightarrow \text { AUDI }\end{array}$ & $\begin{array}{l}\text { DAN } \rightarrow \text { FPR } \\
\text { MVISU } \leftarrow \text { FPR } \\
\text { LVISU } \leftarrow \text { FPR } \\
\text { VAN } \leftarrow \text { DAN } \\
\text { VAN } \leftarrow \text { SN } \\
\text { netgc LVISU } \rightarrow \text { SN } \\
\text { netgc FPL } \rightarrow \text { FPR }\end{array}$ & $\begin{array}{l}- \\
- \\
- \\
- \\
- \\
-\end{array}$ & & $\begin{array}{l}- \\
- \\
- \\
- \\
- \\
- \\
-\end{array}$ & \\
\hline 5 & sFC DMN-VAN & sFC MVISU-DMN & - & & $\begin{array}{l}\text { ip OVIS-SN s2 } \\
\text { ip MVISU-DMN s2 }\end{array}$ & ip CB-SN s2 & InEP DMN & \\
\hline 6 & all best features & (see Table 6.2) & all best features & (see Table 6.2) & all best features & (see Table 6.2) & $\begin{array}{l}\text { InEP DMN } \\
\text { InEP SSM }\end{array}$ & $\begin{array}{l}\text { InEP DAN } \\
\text { InEP CING }\end{array}$ \\
\hline 7 & all best features & (see Table 6.2) & all best features & (see Table 6.2) & all best features & (see Table 6.2) & all best features & (see Table 6.2) \\
\hline 8 & $\begin{array}{l}\text { sFC DMN-VAN } \\
\text { sFC DMN-LSM } \\
\text { sFC DAN-SN }\end{array}$ & $\begin{array}{l}\text { sFC MVISU-DMN } \\
\text { sFC DMN-CB } \\
\text { sFC OVISU-SN }\end{array}$ & $\begin{array}{l}\text { GC NetDegDAN } \\
\text { GC NetDeg LSM }\end{array}$ & GC NetDegAUDI & ip MVISU-DMN s2 & ip MVISU-DMN s4 & InEP DMN & InEP DAN \\
\hline 9 & $\begin{array}{l}\text { sFC DMN-VAN } \\
\text { sFC DMN-LSM } \\
\text { sFC DAN-SN }\end{array}$ & $\begin{array}{l}\text { sFC MVISU-DMN } \\
\text { sFC DMN-CB } \\
\text { sFC OVISU-SN }\end{array}$ & - & & ip CB-SN s2 & ip MVISU-DMN s2 & InEP DMN & InEP DAN \\
\hline
\end{tabular}





\section{Appendix D}

\section{Appendix Chapter 7}

\section{D.1 Approximation of hypothesis likelihood marginalization}

The graph sets in (7.14) contain $2^{L^{2}-1}$ graphs, which quickly becomes a very large as $L$ increases and therefore the marginalization in (7.13) will become computationally expensive. This makes the hypothesis test unfeasible to do in a reasonable time, especially because hypotheses must be tested for groups of subjects.

First of all, note that from the definition of our model in (7.4) and $p(D \mid \mathcal{G})$ in (7.8), we see that the marginalization in (7.13) can be decomposed into $L$ separate marginalizations. Then, we only need to marginalise over $\mathcal{G}_{j}$, i.e. the subgraph which contains connection $e_{j i}$ :

$$
p\left(D^{k} \mid e_{j i}\right) \propto \sum_{\mathcal{G}_{j} \in \mathcal{P}_{j, 1}} p\left(\mathcal{G}_{j}\right) p\left(D^{k} \mid \mathcal{G}_{j}\right)
$$

where $\mathcal{P}_{j, 1}=\left\{\mathcal{G}_{j} \mid e_{j i} \in \mathcal{G}_{j}\right\}$. Note that $p\left(D^{k} \mid e_{j i}\right)$ is now only proportional to the marginalization. This is not an issue, because when we use the proportional marginals from (D.1) to calculate BF in (7.15), it will still result in the correct log-likelihood ratio. 



\section{Bibliography}

Aarsland, D., J. Zaccai, and C. Brayne (2005). "A systematic review of prevalence studies of dementia in Parkinson's disease". In: Movement Disorders 20.10, pp. 1255-1263. ISSN: 08853185. DOI: $10.1002 / \mathrm{mds} .20527$.

Abraham, A et al. (2016). “Deriving reproducible biomarkers from multi-site resting-state data: An Autism-based example". In: Neuroimage. ISSN: 10538119. DOI: 10 . 1016/ j . neuroimage . 2016 . 10.045. arXiv: 1611.06066.

Abrol, A. et al. (2016). "The chronnectome: Evaluating replicability of dynamic connectivity patterns in 7500 resting fMRI datasets". In: Proceedings of the Annual International Conference of the IEEE Engineering in Medicine and Biology Society, EMBS. Vol. 2016-Octob, pp. 5571-5574. ISBN: 9781457702204. DOI: 10.1109/EMBC. 2016.7591989.

Akaike, H. (1974). "A new look at the statistical Model Identification". In: IEEE Transactions on Automatic Control 19.6, pp. 716-723. DOI: 10.1109/TAC.1974.1100705.

Alam, S. and G. R. Kwon (2017). "Alzheimer disease classification using KPCA, LDA, and multikernel learning SVM". In: International Journal of Imaging Systems and Technology 27.2, pp. 133143. ISSN: 10981098. DOI: 10.1002/ima. 22217.

Aldenkamp, A. and J. Arends (2004). “The Relative Influence of Epileptic EEG Discharges, Short Nonconvulsive Seizures, and Type of Epilepsy on Cognitive Function". In: Epilepsia 45.1, pp. 5463. ISSN: 00139580. DOI: 10.1111/j.0013-9580.2004.33403.x.

Allen, E. A. et al. (2014). “Tracking Whole-Brain Connectivity Dynamics in the Resting State". In: Cerebral Cortex 24.3, pp. 663-676. ISSN: 1047-3211. DOI: 10.1093/cercor/bhs352.

Allen, E. A. et al. (2011). "A Baseline for the Multivariate Comparison of Resting-State Networks". In: Frontiers in Systems Neuroscience 5.February, pp. 1-23. ISSN: 1662-5137. DOI: 10.3389/fnsys . 2011.00002.

American Psychiatric Association (2013). Diagnostic and Statistical Manual of Mental Disorders. 5th ed. Washington, DC.: American Psychiatric Association. ISBN: 0-89042-555-8. DOI: $10.1176 /$ appi. books. 9780890425596.

Anderson, J. S., M. A. Ferguson, and J. A. Nielsen (2013). “Functional MRI in Autism”. In: Imaging the Brain in Autism. Ed. by M. F. Casanova, A. S. El-Baz, and J. S. Suri. New York, NY: Springer New York. Chap. 10, pp. 289-323. ISBN: 978-1-4614-6843-1. DOI: 10 . 1007 /978-1-4614-68431_10.

Anderson, J. S. et al. (2011). "Connectivity Gradients Between the Default Mode and Attention Control Networks". In: Brain Connectivity 1.2, pp. 147-157. ISSN: 2158-0014. DOI: 10 . 1089 / brain. 2011.0007.

Andrews-Hanna, J. R. et al. (2007). "Disruption of Large-Scale Brain Systems in Advanced Aging". In: Neuron 56.5, pp. 924-935. ISSN: 08966273. DOI: 10 . 1016/ j . neuron . 2007 . 10 . 038. arXiv: NIHMS150003.

Avelar-Pereira, B. et al. (2017). “Age-related differences in dynamic interactions among default mode, frontoparietal control, and dorsal attention networks during resting-state and interference resolution". In: Frontiers in Aging Neuroscience 9.MAY, pp. 1-15. ISSN: 16634365. DOI: 10 . 3389/fnagi. 2017.00152. 
Avena-Koenigsberger, A., B. Misic, and O. Sporns (2018). "Communication dynamics in complex brain networks". In: Nature Reviews Neuroscience 19.1, pp. 17-33. ISSN: 14710048. DOI: 10.1038/ nrn.2017.149.

Baram, T. Z. (2012). “The Brain, Seizures and Epilepsy Throughout Life: Understanding a Moving Target". In: Epilepsy Currents 12.4s, pp. 7-12. ISSN: 1535-7597. DOI: 10.5698/1535-7511-12 .4s . 7.

Barendse, E. M. et al. (2013). "Working memory deficits in high-functioning adolescents with autism spectrum disorders : neuropsychological and neuroimaging correlates". In: Journal of Neurodevelopmental Disorders, pp. 1-11. DOI: 10.1186/1866-1955-5-14.

Barnett, L., A. B. Barrett, and A. K. Seth (2009). “Granger causality and transfer entropy are equivalent for Gaussian variables". In: Physical Review Letters 103.23. DOI: 10 . 1103 / PhysRevLett . 103. 238701.

Barnett, L. and A. K. Seth (2014). "The MVGC multivariate Granger causality toolbox: A new approach to Granger-causal inference". In: Journal of Neuroscience Methods 223, pp. 50-68. ISSN: 01650270. DOI: $10.1016 / j$. jneumeth. 2013.10.018.

Barrett, A. B. et al. (2012). "Granger Causality Analysis of Steady-State Electroencephalographic Signals during Propofol-Induced Anaesthesia". In: PLOS ONE. DOI: 10 .1371/ journal . pone. 0029072.

Barttfeld, P. et al. (2012). "State-dependent changes of connectivity patterns and functional brain network topology in autism spectrum disorder". In: Neuropsychologia 50.14, pp. 3653-3662. ISSN: 00283932. DOI: $10.1016 / \mathrm{j}$. neuropsychologia.2012.09.047.

Bastin, C. et al. (2012). "Cognitive reserve impacts on inter-individual variability in resting-state cerebral metabolism in normal aging". In: NeuroImage 63.2, pp. 713-722. ISSN: 10538119. DOI: 10.1016/j. neuroimage. 2012.06.074.

Beason-Held, L. L., M. A. Kraut, and S. M. Resnick (2009). "Stability of default-mode network activity in the aging brain". In: Brain Imaging and Behavior 3.2, pp. 123-131. ISSN: 19317557. DOI: 10.1007/s11682-008-9054-z.

Beckmann, C. F. and S. M. Smith (2004). "Probabilistic independent component analysis for functional magnetic resonance imaging." In: IEEE transactions on medical imaging 23.2, pp. 137-52. ISSN: 0278-0062. DOI: 10.1109/TMI . 2003.822821. arXiv: 978-0-87893-286-3.

Beckmann, C. F. et al. (2005). "Investigations into resting-state connectivity using independent component analysis." In: Philosophical transactions of the Royal Society of London. Series B, Biological sciences 360.1457, pp. 1001-13. ISSN: 0962-8436. DOI: 10.1098/rstb.2005.1634.

Beckmann, C. F. et al. (2009). “Group comparison of resting-state FMRI data using multi-subject ICA and dual regression". In: Human Brain Mapping Conference, p. 181.

Beer, J. C. et al. (2019). "Incorporating prior information with fused sparse group lasso: Application to prediction of clinical measures from neuroimages". In: Biometrics 75.4, pp. 1299-1309. DOI: $10.1111 / \mathrm{biom} .13075$.

Bell, A. J. and T. J. Sejnowski (1995). “An Information-Maximization Approach to Blind Separation and Blind Deconvolution". In: Neural Computation 7.6, pp. 1129-1159. ISSN: 0899-7667. DOI: 10 . 1162/neco.1995.7.6.1129. arXiv: arXiv:1511.06440v1.

Belmonte, M. K. (2004). "Autism and Abnormal Development of Brain Connectivity". In: Journal of Neuroscience 24.42, pp. 9228-9231. ISSN: 0270-6474. DOI: 10. 1523/ JNEUROSCI . 3340-04 . 2004.

Bernas, A. (2016). Neurodynamics in functional MRI and its clinical applications. English. PDEng thesis. Technische Universiteit Eindhoven. ISBN: 978-90-444-1444-8.

Bernas, A., A. P. Aldenkamp, and S. Zinger (2018). "Wavelet coherence-based classifier: A restingstate functional MRI study on neurodynamics in adolescents with high-functioning autism". In: Computer Methods and Programs in Biomedicine 154, pp. 143-151. ISSN: 18727565. DOI: 10.1016 / j.cmpb.2017.11.017.

Bernas, A. et al. (2018). "Brain resting-state networks in adolescents with high-functioning autism: Analysis of spatial connectivity and temporal neurodynamics". In: Brain and Behavior 8.2, pp. 110. ISSN: 21623279 . DOI: 10.1002 /brb3. 878. 
Besseling, R. M. H. et al. (2013). "Reduced functional integration of the sensorimotor and language network in rolandic epilepsy". In: NeuroImage: Clinical 2.1, pp. 239-246. ISSN: 22131582. DOI: $10.1016 / \mathrm{j}$.nicl.2013.01.004.

Bildt, A. de et al. (2009). "Evaluation of the ADOS Revised Algorithm: The Applicability in 558 Dutch Children and Adolescents". In: Journal of Autism and Developmental Disorders 39.9, pp. 1350-1358. ISSN: 0162-3257. DOI: 10.1007/s10803-009-0749-9.

Billings, J. et al. (2017). "Instantaneous Brain Dynamics Mapped to a Continuous State Space". In: NeuroImage. ISSN: 10538119. DOI: 10.1101/157115.

Bishop, C. M. (2009). Pattern recognition and machine learning. Springer. ISBN: 978-0387-31073-2.

Biswal, B. et al. (1995). "Functional connectivity in the motor cortex of resting human brain using echo-planar mri". In: Magnetic Resonance in Medicine 34.4, pp. 537-541. ISSN: 07403194. DOI: 10. 1002/mrm. 1910340409.

Blood, A. J. et al. (1999). "Emotional responses to pleasant and unpleasant music correlate with actvity in paralimbic brain regions". In: Nature Neuroscience 2, pp. 382-387. DOI: 10.1038/7299.

Bodner, M. et al. (2012). "Reduction of Seizure Occurence from Exposure to Auditory Stimulation in Individuals with Neurological Handicaps: A Randomized Controlled Trial". In: PLoS One 7.10. DOI: 10.1371 /journal. pone.0045303.

Bos, D. J. et al. (2014). "Developmental differences in higher-order resting-state networks in Autism Spectrum Disorder". In: NeuroImage: Clinical 4, pp. 820-827. ISSN: 22131582. DOI: 10 . 1016/j.nicl.2014.05.007.

Braakman, H. M. et al. (2013). "Frontal lobe connectivity and cognitive impairment in pediatric frontal lobe epilepsy". In: Epilepsia 54.3, pp. 446-454. ISSN: 00139580. DOI: 10.1111/epi.12044.

Bressler, S. L. and A. K. Seth (2011). "Wiener-Granger Causality: A well established methodology". In: NeuroImage 58.2, pp. 323-329. ISSN: 10538119. DOI: 10.1016/j . neuroimage . 2010.02. 059.

Breuer, L. E. M. et al. (2016a). “Cognitive deterioration in adult epilepsy: clinical characteristics of "Accelerated Cognitive Ageing"". In: Acta Neurologica Scandinavica September, pp. 1-7. ISSN: 00016314. DOI: 10.1111/ane.12700.

Breuer, L. E. et al. (2016b). "Cognitive deterioration in adult epilepsy: Does accelerated cognitive ageing exist?" In: Neuroscience and Biobehavioral Reviews 64, pp. 1-11. ISSN: 18737528. DOI: 10 . 1016/j.neubiorev. 2016.02.004.

Breuer, L. E. M. et al. (2019). “Accelerated Cognitive Ageing in Epilepsy: A Neuropsychological Evaluation of Cognitive Deterioration". In: Archives of Clinical Neuropsychology 34.3. ISSN: 18735843. DOI: $10.1093 /$ arclin/acy042.

Brodie, M. J., A. T. Elder, and P. Kwan (2009). “Epilepsy in later life”. In: The Lancet Neurology 8.11, pp. 1019-1030. ISSN: 14744422. DOI: 10.1016/S1474-4422 (09)70240-6.

Buckner, R. L. et al. (2009). “Cortical hubs revealed by intrinsic functional connectivity: Mapping, assessment of stability, and relation to Alzheimer's disease". In: Journal of Neuroscience 29.6, pp. 1860-1873. ISSN: 02706474. DOI: 10.1523/JNEUROSCI . 5062-08. 2009.

Buxton, R. B., E. C. Wong, and L. R. Frank (1998). "Dynamics of blood flow and oxygenation changes during brain activation: The balloon model". In: Magnetic Resonance in Medicine 39.6, pp. 855-864. DOI: $10.1002 / \mathrm{mrm} .1910390602$.

Calhoun, V. D. and T. Adali (2016). "Time-Varying Brain Connectivity in fMRI Data: Whole-brain data-driven approaches for capturing and characterizing dynamic states". In: IEEE Signal Processing Magazine 33.3, pp. 52-66. ISSN: 10535888. DOI: 10.1109/MSP. 2015. 2478915.

Calhoun, V. D. et al. (2014). "The Chronnectome: Time-Varying Connectivity Networks as the Next Frontier in fMRI Data Discovery". In: Neuron 84.2, pp. 262-274. ISSN: 10974199. DOI: 10 . 1016/ j.neuron.2014.10.015.

Cao, W. et al. (2014). "Resting-state functional connectivity in anterior cingulate cortex in normal aging." In: Frontiers in aging neuroscience 6.October, pp. 1-7. ISSN: 1663-4365. DOI: 10 . 3389 / fnagi.2014.00280. 
Catarino, A. et al. (2013). "Task-related functional connectivity in autism spectrum conditions: an EEG study using wavelet transform coherence." In: Molecular autism 4.1, pp. 1-14. ISSN: 20402392. DOI: $10.1186 / 2040-2392-4-1$.

Centers for Disease Control and Prevention (2016). Community Report From the Autism and Developmental Disabilities Monitoring (ADDM) Network. Tech. rep., pp. 1 -51.

Chan, M. Y. et al. (2014). "Decreased segregation of brain systems across the healthy adult lifespan". In: Proceedings of the National Academy of Sciences 111.46, E4997-E5006. ISSN: 0027-8424. DOI: $10.1073 /$ pnas. 1415122111.

Chan, R. C. K. et al. (2008). "Assessment of executive functions: Review of instruments and identification of critical issues". In: Archives of Clinical Neuropsychology 23.2, pp. 201-216. ISSN: 08876177. DOI: 10.1016/j.acn. 2007.08.010. arXiv: 1511.04103.

Chand, G. B. et al. (2017). "Interactions of the Salience Network and Its Subsystems with the Default-Mode and the Central-Executive Networks in Normal Aging and Mild Cognitive Impairment". In: Brain Connectivity 7.7, pp. 401-412. ISSN: 2158-0014. DOI: 10 . 1089/brain . 2017. 0509.

Chang, C. and G. H. Glover (2010). "Time-frequency dynamics of resting-state brain connectivity measured with fMRI". In: NeuroImage 50.1, pp. 81-98. ISSN: 10538119. DOI: 10 . 1016 / j . neuroimage. 2009.12.011.

Chen, A. C. et al. (2013). "Causal interactions between fronto-parietal central executive and default-mode networks in humans". In: Proceedings of the National Academy of Sciences 110.49, pp. 19944-19949. ISSN: 0027-8424. DOI: 10.1073/pnas. 1311772110.

Chen, J. L., V. B. Penhue, and R. J. Zatorre (2008). “Listening to Musical Rhythms Recruits Motor Regions of the Brain". In: Cerebral Cortex 18, pp. 2844-2854. DOI: 10.1093/cercor/bhn042.

Chen, T. et al. (2016). "Distinct Global Brain Dynamics and Spatiotemporal Organization of the Salience Network". In: PLoS Biology 14.6, pp. 1-21. ISSN: 15457885. DOI: 10 . 1371 / journal . pbio.1002469.

Cherkassky, V. L. et al. (2006). "Functional connectivity in a baseline resting-state network in autism." In: Neuroreport 17.16, pp. 1687-1690. ISSN: 0959-4965. DOI: 10 . 1097 / 01 . wnr . $0000239956.45448 .4 c$.

Chickering, D. M. (2002). "Optimal structure identification with greedy search". In: Journal of Machine Learning Research 3, pp. 507-554. DOI: 10.1162/153244303321897717.

Chiuso, A. and G. Pillonetto (2012). "A Bayesian Approach to sparse dynamic network identification". In: Automatica 48.8, pp. 1553-1565. DOI: 10.1016/j . automatica. 2012.05.054.

Choe, A. S. et al. (2015). "Reproducibility and temporal structure in weekly resting-state fMRI over a period of 3.5 years". In: PLOS ONE 10.10, pp. 1-29. ISSN: 19326203. DOI: 10.1371 / journal . pone. 0140134.

Choe, A. S. et al. (2017). "Comparing test-retest reliability of dynamic functional connectivity methods". In: NeuroImage 158, pp. 155-175. ISSN: 10959572. DOI: 10.1016/j . neuroimage. 2017. 07.005.

Churchill, N. W. et al. (2016). "The suppression of scale-free fMRI brain dynamics across three different sources of effort: Aging, task novelty and task difficulty". In: Scientific Reports 6.August, pp. 1-16. ISSN: 20452322. DOI: 10.1038/srep30895.

Ciuciu, P. et al. (2012). "Scale-free and multifractal time dynamics of fmri signals during rest and task". In: Frontiers in Physiology 3.June, pp. 1-18. ISSN: 1664042X. DOI: 10 . 3389/fphys . 2012. 00186. arXiv: 1308.4385.

Ciuciu, P., P. Abry, and B. J. He (2014). "Interplay between functional connectivity and scale-free dynamics in intrinsic fMRI networks". In: NeuroImage 95, pp. 248-263. ISSN: 10959572. DOI: 10 . $1016 / j$. neuroimage . 2014.03.047.

Cole (2010). "Advances and pitfalls in the analysis and interpretation of resting-state FMRI data". In: Frontiers in Systems Neuroscience 4.April, pp. 1-15. ISSN: 16625137. DOI: 10 . 3389/fnsys . 2010. 00008. 
Cole, M. W. et al. (2012). "Global connectivity of prefrontal cortex predicts cognitive control and intelligence". In: Journal of Neuroscience 32.26, pp. 8988-8999. ISSN: 02706474. DOI: 10 . 1523 / JNEUROSCI . 0536-12.2012.

Cook, I. A. et al. (2014). "Do prefrontal midline electrodes provide unique neurophysiologic information in Major Depressive Disorder?" In: Journal of Psychiatric Research 53.1, pp. 69-75. ISSN: 18791379. DOI: 10.1016/j.jpsychires. 2014.01.018.

Coppola, G. et al. (2015). "Mozart's music in children with drug-refractory epileptic encephalopathies". In: Epilepsy and Behavior 50, pp. 18-22. DOI: 10.1016/j · yebeh. 2015.05.038.

Corbetta, M., G. Patel, and G. L. Shulman (2008). "The Reorienting System of the Human Brain: From Environment to Theory of Mind". In: Neuron 58.3, pp. 306-324. ISSN: 08966273. DOI: 10 . 1016/j.neuron.2008.04.017. arXiv: arXiv:1011.1669v3.

Cuijpers, P. et al. (2009). "Psychological treatment of depression in primary care: A meta-analysis". In: British Journal of General Practice 59.559, pp. 120-127. ISSN: 09601643. DOI: 10 . 3399 / bjgp09X395139.

Damaraju, E. et al. (2014). "Dynamic functional connectivity analysis reveals transient states of dysconnectivity in schizophrenia". In: NeuroImage: Clinical 5.July, pp. 298-308. ISSN: 22131582. DOI: $10.1016 / \mathrm{j}$.nicl.2014.07.003.

Damoiseaux, J. S. et al. (2006). "Consistent resting-state networks". In: Proceedings of the National Academy of Sciences 103.37, pp. 13848-13853. DOI: 10.1073/pnas . 0601417103.

Damoiseaux, J. S. et al. (2008). "Reduced resting-state brain activity in the "default network" in normal aging". In: Cerebral Cortex 18.8, pp. 1856-1864. ISSN: 10473211. DOI: 10 . 1093/cercor / bhm207.

Dapretto, M. et al. (2006). "Understanding emotions in others: mirror neuron dysfunction in children with autism spectrum disorders". In: Nature Neuroscience 9.1, pp. 28-30. ISSN: 1097-6256. DOI: $10.1038 / \mathrm{nn} 1611$.

David, O. et al. (2008). “Identifying Neural Drivers with Functional MRI: An Electrophysiological Validation". In: PLoS Biology 6.12, pp. 2683-2697. ISSN: 1545-7885. DOI: 10.1371/journal . pbio. 0060315.

Davis, S. W. et al. (2008). "Qué PASA? the posterior-anterior shift in aging". In: Cerebral Cortex 18.5, pp. 1201-1209. ISSN: 10473211. DOI: 10.1093/cercor/bhm155.

Deco, G. and M. L. Kringelbach (2014). “Great expectations: Using whole-brain computational connectomics for understanding neuropsychiatric disorders". In: Neuron 84.5, pp. 892-905. ISSN: 10974199. DOI: $10.1016 / j$.neuron. 2014.08.034.

Demirci, O. et al. (2009). "Investigation of relationships between fMRI brain networks in the spectral domain using ICA and Granger causality reveals distinct differences between schizophrenia patients and healthy controls". In: Neuroimage 46.2, pp. 419-431. DOI: $10.1016 / \mathrm{j}$. neuroimage . 2009.02.014.

Deshpande, G., P. Santhanam, and X. Hu (2011). “Instantaneous and causal connectivity in resting state brain networks derived from functional MRI data". In: NeuroImage 54.2, pp. 1043-1052.

Deshpande, G. et al. (2009). "Multivariate granger causality analysis of fMRI data". In: Human Brain Mapping 30.4, pp. 1361-1373. ISSN: 10659471. DOI: 10.1002/hbm. 20606.

Deshpande, G. et al. (2013). "Identification of neural connectivity signatures of autism using machine learning". In: Frontiers in Human Neuroscience 7.October, pp. 1-15. ISSN: 1662-5161. DOI: 10.3389/fnhum. 2013.00670.

Deshpande, G. et al. (2015). "Fully connected cascade artificial neural network architecture for attention deficit hyperactivity disorder classification from functional magnetic resonance imaging data". In: IEEE Transactions on Cybernetics 45.12, pp. 2668-2679. ISSN: 21682267. DOI: 10 . 1109 / TCYB. 2014.2379621.

Di, X. and B. B. Biswal (2013). “Dynamic brain functional connectivity modulated by resting-state networks". In: Brain Structure and Function 220.1, pp. 37-46. ISSN: 18632661. DOI: 10 . 1007 / s00429-013-0634-3. arXiv: 15334406. 
Di Martino, A. et al. (2009a). "Autistic Traits in Neurotypical Adults are Related to Cingulo-Insular Functional Connectivity". In: The American journal of psychiatry 166.8, pp. 891-899. ISSN: 0002953X. DOI: 10.1176/appi.ajp. 2009.08121894.

Di Martino, A. et al. (2009b). "Functional Brain Correlates of Social and Nonsocial Processes in Autism Spectrum Disorders: An Activation Likelihood Estimation Meta-Analysis". In: Biological Psychiatry 65.1, pp. 63-74. ISSN: 00063223. DOI: 10.1016/j . biopsych.2008.09.022.

Dipasquale, O. et al. (2017). "Comparing resting state fMRI de-noising approaches using multiand single-echo acquisitions". In: PLoS ONE 12.3, pp. 1-25. ISSN: 19326203. DOI: 10 . 1371 / journal. pone. 0173289.

Dis, E. A. M. van et al. (2020). "Long-term Outcomes of Cognitive Behavioral Therapy for AnxietyRelated Disorders". In: JAMA Psychiatry 77.3, pp. 265-273. ISSN: 2168-622X. DOI: 10 . 1001 / jamapsychiatry.2019.3986.

Douw, L et al. (2016). "State-dependent variability of dynamic functional connectivity between frontoparietal and default network relates to cognitive flexibility". In: Neuroscience 339, pp. 12 21. ISSN: 03064522. DOI: $10.1016 / \mathrm{j}$.neuroscience.2016.09.034.

Drachman, D. A. (2005). “Do we have brain to spare?" In: Neurology 64.12, pp. 2004-2005. ISSN: 00283878. DOI: 10.1212/01. WNL.0000166914.38327.BB.

Du, Y., Z. Fu, and V. D. Calhoun (2018). "Classification and Prediction of Brain Disorders Using Functional Connectivity: Promising but Challenging". In: Frontiers in Neuroscience 12.August. ISSN: 1662-453X. DOI: 10.3389/fnins . 2018.00525.

Ebisch, S. J. H. et al. (2011). "Altered intrinsic functional connectivity of anterior and posterior insula regions in high-functioning participants with autism spectrum disorder". In: Human Brain Mapping 32.7, pp. 1013-1028. ISSN: 10659471. DOI: 10.1002/hbm. 21085.

Eickhoff, S. B., B. T. Yeo, and S. Genon (2018). "Imaging-based parcellations of the human brain". In: Nature Reviews Neuroscience 19.11, pp. 672-686. ISSN: 14710048. DOI: 10.1038/s41583-0180071-7.

Elger, C. E., C. Helmstaedter, and M. Kurthen (2004). "Review Chronic epilepsy and cognition". In: Neurology 3.November, pp. 663-672.

Esposito, F. et al. (2002). "Spatial independent component analysis of functional MRI time-series: To what extent do results depend on the algorithm used?" In: Human Brain Mapping 16.3, pp. 146-157. ISSN: 1065-9471. DOI: 10.1002/hbm. 10034.

Evans, J. W. et al. (2015). “Separating slow BOLD from non-BOLD baseline drifts using multi-echo fMRI”. In: NeuroImage 105, pp. 189-197. ISSN: 10538119. DOI: 10.1016/j . neuroimage . 2014 . 10. 051.

Ezaki, T. et al. (2020). "Closer to critical resting-state neural dynamics in individuals with higher fluid intelligence". In: Communications Biology 3.1, p. 52. ISSN: 2399-3642. DOI: 10.1038/s42003020-0774-y.

Farrant, K. and L. Q. Uddin (2015). "Asymmetric development of dorsal and ventral attention networks in the human brain". In: Developmental Cognitive Neuroscience 12, pp. 165-174. ISSN: 18789307. DOI: 10.1016/j.dcn.2015.02.001. arXiv: 15334406.

Farràs-Permanyer, L., J. Guàrdia-Olmos, and M. Peró-Cebollero (2015). “Mild cognitive impairment and fMRI studies of brain functional connectivity: the state of the art". In: Frontiers in Psychology 6.August, pp. 1-18. ISSN: 1664-1078. DOI: 10.3389/f psyg. 2015. 01095.

Ferreira, L. K. and G. F. Busatto (2013). "Resting-state functional connectivity in normal brain aging". In: Neuroscience \& Biobehavioral Reviews 37.3, pp. 384-400. ISSN: 01497634. DOI: 10.1016/ j.neubiorev. 2013.01.017.

Ferreira, L. K. et al. (2016). "Aging effects on whole-brain functional connectivity in adults free of cognitive and psychiatric disorders". In: Cerebral Cortex 26.9, pp. 3851-3865. ISSN: 14602199. DOI: $10.1093 /$ cercor/bhv190. 
Filippini, N. et al. (2009). "Distinct patterns of brain activity in young carriers of the APOEepsilon4 allele." In: Proceedings of the National Academy of Sciences 106.17, pp. 7209-7214. ISSN: 1091-6490. DOI: 10.1073/pnas.0811879106.

Fonov, V. et al. (2011). "Unbiased average age-appropriate atlases for pediatric studies". In: NeuroImage 54.1, pp. 313-327. ISSN: 10538119. DOI: 10 . 1016/ j . neuroimage . 2010 . 07 . 033. arXiv: NIHMS150003.

Fornito, A., A. Zalesky, and M. Breakspear (2015). "The connectomics of brain disorders". In: Nature Reviews Neuroscience 16.3, pp. 159-172. ISSN: 1471-003X. DOI: 10.1038/nrn3901.

Fox, M. D. and M. E. Raichle (2007). "Spontaneous fluctuations in brain activity observed with functional magnetic resonance imaging". In: Nature Reviews Neuroscience 8.9, pp. 700-711. ISSN: 1471003X. DOI: $10.1038 / \mathrm{nrn} 2201$.

Fox, M. D. et al. (2006). "Spontaneous neuronal activity distinguishes human dorsal and ventral attention systems". In: Proceedings of the National Academy of Sciences 103.26, pp. 10046-10051. ISSN: 0027-8424. DOI: 10.1073/pnas. 0604187103.

Franzmeier, N. et al. (2017). "Resting-state connectivity of the left frontal cortex to the default mode and dorsal attention network supports reserve in mild cognitive impairment". In: Frontiers in Aging Neuroscience 9.August, pp. 1-11. ISSN: 16634365. DOI: 10.3389/fnagi.2017.00264.

Franzmeier, N. et al. (2018). "The left frontal cortex supports reserve in aging by enhancing functional network efficiency". In: Alzheimer's Research and Therapy 10.1. ISSN: 17589193. DOI: 10 . 1186/s13195-018-0358-y.

Friston, K. J., L. Harrison, and W. Penny (2003). “Dynamic causal modelling”. In: Neuroimage 19.4, pp. 1273-1302. DOI: 10.1016/S1053-8119(03)00202-7.

Friston, K., R. Moran, and A. K. Seth (2013). "Analysing connectivity with Granger causality and dynamic causal modelling". In: Current Opinion in Neurobiology 23.2, pp. 172-178. ISSN: 09594388. DOI: $10.1016 / j$. conb.2012.11.010.

Friston, K. J. (2011). “Functional and Effective Connectivity: A Review”. In: Brain Connectivity 1.1, pp. 13-36. ISSN: 2158-0014. DOI: 10.1089/brain.2011.0008.

Gauthier, S. et al. (2006). "Mild cognitive impairment". In: The Lancet 367.9518, pp. 1262-1270. ISSN: 01406736. DOI: 10.1016/S0140-6736(06)68542-5. arXiv: 5.

Geweke, J. (1984). "Measures of conditional linear dependence and feedback between time series". In: Journal of the American Statistical Association 79.388, pp. 907-915. DOI: 10 . 1080 /01621459 . 1984.10477110.

Gilliam, F. G. et al. (2004). “Depression in Epilepsy: Ignoring Clinical Expression of Neuronal Network Dysfunction?" In: Epilepsia 45.s2, pp. 28-33. ISSN: 0013-9580. DOI: 10 .1111/ j .0013$9580.2004 .452005 . x$.

Gonzalez-Castillo, J. et al. (2019). "Imaging the spontaneous flow of thought: Distinct periods of cognition contribute to dynamic functional connectivity during rest". In: NeuroImage 202.August, pp. 1-14. ISSN: 10538119. DOI: 10.1016/j . neuroimage.2019.116129.

Gotts, S. J. et al. (2013). "The perils of global signal regression for group comparisons: A case study of Autism Spectrum Disorders". In: Frontiers in Human Neuroscience 7.July, pp. 1-20. ISSN: 16625161. DOI: $10.3389 /$ fnhum. 2013.00356.

Grady, C. L. et al. (2006). "Age-related changes in brain activity across the adult lifespan". In: Journal of Cognitive Neuroscience 18.2, pp. 227-241. ISSN: 15308898. DOI: 10.1162/ jocn. 2006.18. 2.227.

Granger, C. W. J. (1969). "Investigating Causal Relations by Econometric Models and Crossspectral Methods". In: The Econometric Society 37.3, pp. 424-438. DOI: 10.2307/1912791.

Greicius, M. D. et al. (2004). "Default-mode network activity distinguishes Alzheimer's disease from healthy aging: evidence from functional MRI." In: Proc. Natl. Acad. Sci. U. S. A. 101.13, pp. 4637-4642. ISSN: 0027-8424. DOI: 10.1073/pnas . 0308627101.

Greicius, M. D. et al. (2007). “Resting-State Functional Connectivity in Major Depression: Abnormally Increased Contributions from Subgenual Cingulate Cortex and Thalamus". In: Biological 
Psychiatry 62.5, pp. 429-437. ISSN: 00063223. DOI: 10 . 1016/ j . biopsych . 2006 . 09 . 020. arXiv: NIHMS150003.

Grinsted, A., J. C. Moore, and S. Jevrejeva (2004). "Application of the cross wavelet transform and wavelet coherence to geophysical time series". In: Nonlinear Processes in Geophysics 11.5/6, pp. 561-566. ISSN: 1023-5809. DOI: 10.5194/npg-11-561-2004.

Guo, W. et al. (2015). "Increased cerebellar-default-mode-network connectivity in drug-naive major depressive disorder at rest". In: Medicine (United States) 94.9, e560. ISSN: 15365964. DOI: 10 . 1097/MD.0000000000000560.

Hafkemeijer, A., J. van der Grond, and S. A. Rombouts (2012). "Imaging the default mode network in aging and dementia". In: Biochimica et Biophysica Acta-Molecular Basis of Disease 1822.3, pp. 431-441. ISSN: 09254439. DOI: 10.1016/j . bbadis . 2011.07.008.

Hanson, C. et al. (2013). "Atypical effective connectivity of social brain networks in individuals with autism." In: Brain connectivity 3.6, pp. 578-89. ISSN: 2158-0022. DOI: 10. 1089/brain. 2013. 0161.

Hassan Saleh, I. E. (2011). "Resting State fMRI Time Series : from Noise Correction to Functional Recovery". PhD thesis. Sapienza Università Di Roma.

Helmstaedter, C. and C. E. Elger (2009). "Chronic temporal lobe epilepsy: A neurodevelopmental or progressively dementing disease". In: Brain 132.10, pp. 2822-2830. ISSN: 00068950. DOI: 10 . 1093/brain/awp182.

Helmstaedter, C. et al. (2014). "Disentangling the relationship between epilepsy and its behavioral comorbidities - The need for prospective studies in new-onset epilepsies". In: Epilepsy and Behavior 31, pp. 43-47. ISSN: 15255050. DOI: 10.1016/j. yebeh. 2013.11.010.

Herculano-Houzel, S. (2009). "The human brain in numbers: A linearly scaled-up primate brain". In: Frontiers in Human Neuroscience 3.November, pp. 1-11. ISSN: 16625161. DOI: 10.3389/neuro. 09.031.2009.

Hermann, B. et al. (2008). "Growing old with epilepsy: the neglected issue of cognitive and brain health in aging and elder persons with chronic epilepsy." In: Epilepsia 49.5, pp. 731-40. ISSN: 0013-9580. DOI: $10.1111 / j .1528-1167.2007 .01435 . x$.

Hermann, B. P. et al. (2006). "Cognitive prognosis in chronic temporal lobe epilepsy". In: Annals of Neurology 60.1, pp. 80-87. ISSN: 03645134. DOI: 10.1002/ana. 20872.

Heunis, S. et al. (2018). "Neu3CA-RT: A framework for real-time fMRI analysis". In: Psychiatry Research - Neuroimaging 282, pp. 90-102. ISSN: 18727506. DOI: $10.1016 /$ j . pscychresns . 2018. 09.008.

Heuvel, M. P. van den and H. E. Hulshoff Pol (2010). “Exploring the brain network: A review on resting-state fMRI functional connectivity". In: European Neuropsychopharmacology 20.8, pp. 519534. ISSN: 0924977X. DOI: 10.1016/j . euroneuro. 2010.03.008.

Hindriks, R. et al. (2016). "Can sliding-window correlations reveal dynamic functional connectivity in resting-state fMRI?" In: NeuroImage 127, pp. 242-256. ISSN: 10959572. DOI: $10.1016 / \mathrm{j}$. neuroimage.2015.11.055.

Hochberg, Y. and A. C. Tamhane (1987). Multiple comparison procedures. New York: Wiley.

Hof, P. M. J. Van den et al. (2013). "Identification of dynamic models in complex networks with prediction error methods - basic methods for consistent module estimates". In: Automatica 49.10, pp. 2994-3006. DOI: 10.1016/j . automatica.2013.07.011.

Holdnack, J. A. et al. (2013). Predicting Premorbid Ability for WAIS-IV, WMS-IV and WASI-II. Elsevier Inc., pp. 217-278. ISBN: 9780123869340. DOI: 10.1016/B978-0-12-386934-0.00005-5.

Hoptman, M. J. et al. (2010). "Amplitude of low-frequency oscillations in schizophrenia: A resting state fMRI study". In: Schizophrenia Research 117.1, pp. 13-20. ISSN: 09209964. DOI: 10.1016/ j . schres.2009.09.030.

Hramov, A. E. et al. (2015). Wavelets in Neuroscience. Springer Series in Synergetics. Springer. ISBN: 9783540381648. 
Huang, C.-C. et al. (2017). "Effective Connectivity in Depression". In: Biological Psychiatry: Cognitive Neuroscience and Neuroimaging 3.2, pp. 187-197. ISSN: 24519022. DOI: 10.1016/ j . bpsc . 2017. 10.004.

Huang, X. et al. (2019). "Progress in psychoradiology, the clinical application of psychiatric neuroimaging". In: The British Journal of Radiology 92.1101, p. 20181000. ISSN: 0007-1285. DOI: 10 . 1259/bjr. 20181000.

Hull, J. V. et al. (2017). "Resting-state functional connectivity in autism spectrum disorders: A review". In: Frontiers in Psychiatry 7.JAN. ISSN: 16640640. DOI: 10 .3389/fpsyt.2016.00205.

Hutchison, R. M. et al. (2013). “Dynamic functional connectivity: Promise, issues, and interpretations". In: NeuroImage 80, pp. 360-378.

Hyvärinen, A (1999). "Fast and robust fixed-point algorithms for independent component analysis." In: IEEE transactions on neural networks 10.3, pp. 626-34. ISSN: 1045-9227. DOI: 10 . 1109/72. 761722.

Hyvärinen, A., J. Karhunen, and E. Oja (2001). Independent Component Analysis. New York: Wiley. Igelström, K. M., T. W. Webb, and M. S. A. Graziano (2016). "Functional Connectivity Between the Temporoparietal Cortex and Cerebellum in Autism Spectrum Disorder". In: Cerebral Cortex 27.4, pp. 2617-2627. ISSN: 1047-3211. DOI: $10.1093 /$ cercor/bhw079.

Iidaka, T. (2015). "Resting state functional magnetic resonance imaging and neural network classified autism and control". In: Cortex 63, pp. 55-67. ISSN: 19738102. DOI: 10 . 1016/ j . cortex . 2014.08 .011$.

Indic, P. and J. Narayanan (2011). "Wavelet based algorithm for the estimation of frequency flow from electroencephalogram data during epileptic seizure". In: Clinical Neurophysiology 122.4, pp. 680-686. ISSN: 13882457. DOI: 10.1016/ j . clinph.2010.10.030.

Insabato, A., G. Deco, and M. Gilson (2019). "Imaging Connectomics and the Understanding of Brain Diseases". In: Advances in experimental medicine and biology. Vol. 1192. Springer Singapore, pp. 139-158. ISBN: 9789813297210. DOI: 10.1007/978-981-32-9721-0_8.

Jenkinson, M. et al. (2002). "Improved Optimization for the Robust and Accurate Linear Registration and Motion Correction of Brain Images". In: NeuroImage 17.2, pp. 825-841. ISSN: 10538119. DOI: $10.1006 /$ nimg . 2002.1132.

Kaiser, A. and T. Schreiber (2002). "Information transfer in continuous processes". In: Physica D: Nonlinear Phenomena 166.1-2, pp. 43-62. DOI: 10.1016/S0167-2789(02) 00432-3.

Kaiser, R. H. et al. (2016). "Dynamic Resting-State Functional Connectivity in Major Depression". In: Neuropsychopharmacology 41.7, pp. 1822-1830. ISSN: 1740634X. DOI: 10.1038/npp. 2015. 352.

Kana, R. K. et al. (2014). "Brain connectivity in autism." In: Frontiers in human neuroscience 8.June, p. 349. ISSN: 1662-5161. DOI: 10.3389/fnhum. 2014.00349.

Kana, R. K. et al. (2015). "Aberrant functioning of the theory-of-mind network in children and adolescents with autism". In: Molecular Autism 6.1, pp. 1-12. ISSN: 2040-2392. DOI: 10 . 1186 / s13229-015-0052-x.

Kass, R. E. and A. E. Raftery (1995). "Bayes Factors." In: Journal of the American Statistical Society 90.430, pp. 773-795.

Keown, C. L. et al. (2017). "Network Organization Is Globally Atypical in Autism: A Graph Theory Study of Intrinsic Functional Connectivity". In: Biological Psychiatry: Cognitive Neuroscience and Neuroimaging 2.1, pp. 66-75. ISSN: 24519022. DOI: 10.1016/j . bpsc. 2016.07.008.

Kim, J. et al. (2016). "Deep neural network with weight sparsity control and pre-training extracts hierarchical features and enhances classification performance: Evidence from whole-brain resting-state functional connectivity patterns of schizophrenia". In: NeuroImage 124, pp. 127-146. ISSN: 10959572. DOI: $10.1016 / \mathrm{j}$. neuroimage . 2015.05.018.

Klooster, D. C. (2019). "The potential of multimodal neuroimaging to personalize transcranial magnetic stimulation treatment protocols". PhD thesis. Eindhoven: Ghent University; Technische Universiteit Eindhoven. ISBN: 9789463239011. 
Klugah-Brown, B. et al. (2019). "Altered structural and causal connectivity in frontal lobe epilepsy". In: BMC Neurology 19.1, pp. 1-9. ISSN: 14712377. DOI: 10.1186/s12883-019-1300-z.

Koelsch, S. et al. (2005). "Adults and children processing music: An fMRI study". In: Neuroimage 25.4, pp. 1068-1076. DOI: $10.1016 / \mathrm{j}$. neuroimage. 2004.12 .050$.

Koppe, G. et al. (2019). "Identifying nonlinear dynamical systems via generative recurrent neural networks with applications to fMRI". In: PLOS Computational Biology 15.8, pp. 1-35. DOI: 10 . 1371/journal.pcbi.1007263.

Koshino, H. et al. (2008). "fMRI investigation of working memory for faces in autism: Visual coding and underconnectivity with frontal areas". In: Cerebral Cortex 18.2, pp. 289-300. ISSN: 10473211. DOI: $10.1093 /$ cercor/bhm054.

Koush, Y. et al. (2013). “Connectivity-based neurofeedback: Dynamic causal modeling for realtime fMRI". In: NeuroImage 81, pp. 422-430. ISSN: 10538119. DOI: 10.1016/j . neuroimage . 2013. 05.010.

Kriegeskorte, N. and P. K. Douglas (2018). "Cognitive computational neuroscience". In: Nature Neuroscience 21.9, pp. 1148-1160. ISSN: 15461726. DOI: 10.1038/s41593-018-0210-5. arXiv: 1807.11819.

Kruijs, S. J.M.V. D. et al. (2014). "Resting-state networks and dissociation in psychogenic nonepileptic seizures". In: Journal of Psychiatric Research 54, pp. 126-133. ISSN: 0022-3956. DOI: 10 . 1016/j.jpsychires.2014.03.010.

Ktena, S. I. et al. (2018). "Metric learning with spectral graph convolutions on brain connectivity networks". In: NeuroImage 169, pp. 431-442. ISSN: 10959572. DOI: 10 . 1016/ j . neuroimage 2017. 12.052.

Kundu, P. et al. (2012). "Differentiating BOLD and non-BOLD signals in fMRI time series using multi-echo EPI". In: NeuroImage 60.3, pp. 1759-1770. ISSN: 1053-8119. DOI: 10 . 1016 / j . neuroimage.2011.12.028.

Kundu, P. et al. (2017). "Multi-Echo fMRI: A Review of Applications in fMRI Denoising and Analysis of BOLD Signals". In: NeuroImage 154, pp. 1-22. ISSN: 10538119. DOI: 10. 1016/j . neuroimage . 2017.03 .033$.

La Corte, V. et al. (2016). “Cognitive Decline and Reorganization of Functional Connectivity in Healthy Aging: The Pivotal Role of the Salience Network in the Prediction of Age and Cognitive Performances". In: Frontiers in Aging Neuroscience 8.August, pp. 1-12. ISSN: 1663-4365. DOI: 10 . 3389/fnagi. 2016.00204.

Laird, A. R. et al. (2011). "Behavioral Interpretations of Intrinsic Connectivity Networks". In: Journal of Cognitive Neuroscience 23.12, pp. 4022-4037. ISSN: 0898-929X. DOI: 10.1162/jocn_a_00077.

Lammers, M. et al. (1995). "Monotherapy or Poly therapy for Epilepsy Revisited: A Quantitative Assessment". In: Epilepsia 36.5, pp. 440-446. ISSN: 0013-9580. DOI: 10.1111/j .1528-1157.1995. tb00484.x.

Lan, P. S. et al. (2020). "Imaging brain function with simultaneous BOLD and viscoelasticity contrast: fMRI/fMRE". In: NeuroImage 211, pp. 1-10. ISSN: 10959572. DOI: 10 .1016/ j . neuroimage . 2020.116592.

Lau, W. K., M. K. Leung, and B. W. Lau (2019). “Resting-state abnormalities in Autism Spectrum Disorders: A meta-analysis". In: Scientific Reports 9.1, pp. 1-8. ISSN: 20452322. DOI: 10 . 1038 / s41598-019-40427-7.

Lazar, A. S., Z. I. Lazar, and D. J. Dijk (2015). "Circadian regulation of slow waves in human sleep: Topographical aspects". In: NeuroImage 116, pp. 123-134. ISSN: 10959572. DOI: 10 . 1016/ j.neuroimage.2015.05.012.

Lee, J. M. L. et al. (2016). “Abnormalities of Inter- and Intra-Hemispheric Functional Connectivity in Autism Spectrum Disorders: A Study Using the Autism Brain Imaging Data Exchange Database". In: Frontiers in Neuroscience 10.May, pp. 1-11. ISSN: 1662-453X. DOI: 10.3389/fnins. 2016.00191. 
Leonardi, N. et al. (2014). "Disentangling dynamic networks: Separated and joint expressions of functional connectivity patterns in time". In: Human Brain Mapping 35.12, pp. 5984-5995. ISSN: 10970193. DOI: $10.1002 / \mathrm{hbm} .22599$.

Leppik, I. E. and A. K. Birnbaum (2010). "Epilepsy in the elderly". In: Annals of the New York Academy of Sciences 1184.1, pp. 208-224. ISSN: 00778923. DOI: 10.1111/ j . 1749-6632 . 2009. 05113.x.

Levy, R. (1994). “Aging-Associated Cognitive Decline". In: International Psychogeriatrics 6.1, pp. 6368. ISSN: 1041-6102. DOI: $10.1017 /$ S1041610294001626.

Li, H. J. et al. (2015). "Putting age-related task activation into large-scale brain networks: A metaanalysis of $114 \mathrm{fMRI}$ studies on healthy aging". In: Neuroscience and Biobehavioral Reviews 57.16, pp. 156-174. ISSN: 18737528. DOI: 10.1016/j . neubiorev . 2015.08.013.

Li, J. et al. (2019). "Global signal regression strengthens association between resting-state functional connectivity and behavior". In: NeuroImage 196.February, pp. 126-141. ISSN: 10959572. DOI: $10.1016 / j$. neuroimage 2019.04 .016 .

Liao, W. et al. (2010). "Altered functional connectivity and small-world in mesial temporal lobe epilepsy". In: PLOS ONE 5.1, pp. 27-29. ISSN: 19326203. DOI: 10.1371/journal . pone.0008525.

Liégeois, R. et al. (2019). "Resting brain dynamics at different timescales capture distinct aspects of human behavior". In: Nature Communications 10.1, p. 2317. ISSN: 2041-1723. DOI: 10 . 1038 / s41467-019-10317-7.

Liu, X. et al. (2018). "Subcortical evidence for a contribution of arousal to fMRI studies of brain activity". In: Nature Communications 9.1, pp. 1-10. ISSN: 20411723. DOI: 10 . 1038/s41467-01702815-3.

Liu, Y. et al. (2008). "Disrupted small-world networks in schizophrenia". In: Brain 131.4, pp. 945961. ISSN: 14602156. DOI: 10.1093/brain/awn018.

Ljung, L. (1999). System identification: Theory for the User. Englewood Cliffs, NJ: Prentice-Hall.

Lombardo, M. V. et al. (2016). "Improving effect size estimation and statistical power with multiecho fMRI and its impact on understanding the neural systems supporting mentalizing". In: NeuroImage 142, pp. 55-66. ISSN: 10959572. DOI: 10.1016/j . neuroimage.2016.07.022.

Londei, A. et al. (2006). "A new method for detecting causality in fMRI data of cognitive processing". In: Cognitive Processes 7.1, pp. 42-52. DOI: 10.1007/s10339-005-0019-5.

Lowe, M., B. Mock, and J. Sorenson (1998). "Functional Connectivity in Single and Multislice Echoplanar Imaging Using Resting-State Fluctuations". In: NeuroImage 7.2, pp. 119-132. ISSN: 10538119. DOI: 10.1006/nimg. 1997.0315.

Lui, S. et al. (2016). "Psychoradiology: The Frontier of Neuroimaging in Psychiatry". In: Radiology 281.2, pp. 357-372. ISSN: 0033-8419. DOI: 10.1148/radiol. 2016152149.

Luo, N. et al. (2020). "Age-related structural and functional variations in 5,967 individuals across the adult lifespan". In: Human Brain Mapping 41.7, pp. 1725-1737. ISSN: 1065-9471. DOI: 10 . 1002 / hbm. 24905.

Lustig, C. et al. (2003). "Functional deactivations: Change with age and dementia of the Alzheimer type". In: Proceedings of the National Academy of Sciences 100.24, pp. 14504-14509. ISSN: 00278424. DOI: $10.1073 /$ pnas . 2235925100.

Ly, M. et al. (2014). "Midlife measurements of white matter microstructure predict subsequent regional white matter atrophy in healthy adults". In: Human Brain Mapping 35.5, pp. 2044-2054. ISSN: 10970193. DOI: 10.1002/hbm. 22311.

Madeo, D. and C. Mocenni (2015). "Game Interactions and Dynamics on Networked Populations". In: IEEE Transactions on Automatic Control 60.7, pp. 1801-1810. ISSN: 00189286. DOI: 10 . 1109 / TAC. 2014.2384755.

Madeo, D. et al. (2017). “An Evolutionary Game Theory Model of Spontaneous Brain Functioning". In: Scientific Reports 7.1, pp. 1-11. ISSN: 2045-2322. DOI: 10.1038/s41598-017-15865-w. 
Mamah, D. et al. (2019). “White matter integrity in schizophrenia and bipolar disorder: Tract- and voxel-based analyses of diffusion data from the Connectom scanner". In: NeuroImage: Clinical 21, pp. 1-10. ISSN: 22131582. DOI: 10.1016/j . nicl. 2018.101649.

Mantini, D et al. (2007). "Electrophysiological signatures of resting state networks in the human brain." In: Proceedings of the National Academy of Sciences 104.32, pp. 13170-5. ISSN: 0027-8424. DOI: $10.1073 /$ pnas. 0700668104.

Marek, S. and N. U. F. Dosenbach (2018). "The frontoparietal network: function, electrophysiology, and importance of individual precision mapping". In: Dialogues in Clinical Neuroscience 20.2, pp. 133-140.

Marreiros, A. C., S. J. Kiebel, and K. J. Friston (2008). "Dynamic causal modelling for fMRI: a two-state model". In: Neuroimage 39.1, pp. 269-278. DOI: 10.1016/j . neuroimage . 2007 . 08. 019.

Marstaller, L. et al. (2015). "Aging and large-scale functional networks: White matter integrity, gray matter volume, and functional connectivity in the resting state". In: Neuroscience 290, pp. 369378. ISSN: 18737544. DOI: $10.1016 / j$.neuroscience. 2015.01.049.

Materassi, D. and M. Salapaka (2012). "On the Problem of Reconstructing an Unknown Topology via Locality Properties of the Wiener Filter". In: IEEE interactions on Automatic Control 57.7, pp. 1765-1777. DOI: 10.1109/TAC.2012.2183170.

McQuarrie, A. D. R. and C.-L. Tsai (1998). Regression and time series model selection. Singapore: World Scientific.

Menon, V. and L. Q. Uddin (2010). "Saliency, switching, attention and control: a network model of insula function." In: Brain structure \& function 214.5-6, pp. 655-667. ISSN: 18632661. DOI: 10 . 1007/s00429-010-0262-0. arXiv: arXiv:1011.1669v3.

Meszlényi, R. J., K. Buza, and Z. Vidnyánszky (2017). "Resting State fMRI Functional ConnectivityBased Classification Using a Convolutional Neural Network Architecture". In: Frontiers in Neuroinformatics 11.October, pp. 1-12. ISSN: 1662-5196. DOI: 10 . 3389/fninf . 2017 .00061. arXiv: 1707.06682.

Mezer, A. et al. (2009). "Cluster analysis of resting-state fMRI time series". In: NeuroImage 45.4, pp. 1117-1125. ISSN: 10538119. DOI: 10.1016/j . neuroimage. 2008.12.015.

Miller, E. K. and J. D. Cohen (2001). “An Integrative Theory of Prefrontal Cortex Function". In: Annual Review of Neuroscience 24, pp. 167-202. DOI: 10.1146/annurev . neuro. 24.1.167.

Miller, R. L. et al. (2015). "Multidimensional frequency domain analysis of full-volume fmri reveals significant effects of age, gender and mental illness on the spatiotemporal organization of resting-state brain activity". In: Frontiers in Neuroscience 9.May, pp. 1-19. ISSN: 1662453X. DOI: 10.3389 /fnins . 2015.00203.

Monk, C. et al. (2009). "Abnormalities of intrinsic functional connectivity in autism spectrum disorders," in: Neuroimage 47.2, pp. 764-772. DOI: 10.1016/j . neuroimage .2009.04 . 069.

Morf, M. et al. (1978). "Recursive multichannel maximum entropy spectral estimation". In: IEEE Trans. Geosci. Electron 16.2, pp. 85-94.

Müller, R. A. et al. (2011). "Underconnected, but how? A survey of functional connectivity MRI studies in autism spectrum disorders". In: Cerebral Cortex 21.10, pp. 2233-2243. ISSN: 10473211. DOI: $10.1093 /$ cercor/bhq296.

Murphy, K. and M. D. Fox (2017). "Towards a consensus regarding global signal regression for resting state functional connectivity MRI". In: NeuroImage 154, pp. 169-173. ISSN: 10959572. DOI: $10.1016 / j$. neuroimage. 2016.11.052.

$\mathrm{Ng}$, K. K. et al. (2016). "Reduced functional segregation between the default mode network and the executive control network in healthy older adults: A longitudinal study". In: NeuroImage 133, pp. 321-330. ISSN: 10959572. DOI: 10.1016/j . neuroimage. 2016.03.029.

Nichols, T. E. and A. P. Holmes (2002). "Nonparametric permutation tests for functional neuroimaging: a primer with examples." In: Human brain mapping 15.1, pp. 1-25. ISSN: 1065-9471. DOI: $10.1002 / \mathrm{hbm} .1058$. 
Nickerson, L. D. et al. (2017). “Using dual regression to investigate network shape and amplitude in functional connectivity analyses". In: Frontiers in Neuroscience 11.Mars, pp. 1-18. ISSN: 1662453X. DOI: 10.3389/fnins. 2017.00115.

Nielsen, J. A. et al. (2013). "Multisite functional connectivity MRI classification of autism: ABIDE results". In: Frontiers in Human Neuroscience 7.September, pp. 1-12. ISSN: 1662-5161. DOI: 10 . 3389/fnhum. 2013.00599. arXiv: 1611.06066.

Nieuwenhuis, M. et al. (2012). "Classification of schizophrenia patients and healthy controls from structural MRI scans in two large independent samples". In: NeuroImage 61.3, pp. 606-612. ISSN: 10538119. DOI: $10.1016 / \mathrm{j}$. neuroimage . 2012.03.079.

Nomi, J. S. and L. Q. Uddin (2015). "Developmental changes in large-scale network connectivity in autism". In: NeuroImage: Clinical 7, pp. 732-741. ISSN: 22131582. DOI: 10.1016/ j . nicl . 2015. 02.024. arXiv: 15334406.

Nomi, J. S. et al. (2016). "Chronnectomic Patterns and Neural Flexibility Underlie Executive Function". In: NeuroImage 147.October, pp. 861-871. ISSN: 10538119. DOI: 10 . 1016/ j . neuroimage . 2016.10.026.

O'Connor, E. E. and T. A. Zeffiro (2019). “Why is clinical fMRI in a resting state?" In: Frontiers in Neurology 10.April, pp. 1-8. ISSN: 16642295. DOI: 10.3389/fneur. 2019.00420.

Ogawa, S. et al. (1990). "Brain magnetic resonance imaging with contrast dependent on blood oxygenation". In: Proceedings of the National Academy of Sciences 87.24, pp. 9868-9872. DOI: 10 . 1073/pnas. 87.24.9868.

Olson, I. R., A. Plotzker, and Y. Ezzyat (2007). “The Enigmatic temporal pole: A review of findings on social and emotional processing". In: Brain 130.7, pp. 1718-1731. ISSN: 00068950. DOI: 10 . 1093/brain/awm052.

Onoda, K., M. Ishihara, and S. Yamaguchi (2012a). "Aging effects on functional connectivity within and between resting-state networks". In: 2012 ICME International Conference on Complex Medical Engineering, CME 2012 Proceedings 8.978, pp. 119-124. DOI: 10.1109/ICCME. 2012.6275599.

- (2012b). "Decreased Functional Connectivity by Aging Is Associated with Cognitive Decline". In: Journal of Cognitive Neuroscience 24.11, pp. 2186-2198.

Palop, J. J. and L. Mucke (2009). "Epilepsy and cognitive impairments in alzheimer disease". In: Archives of Neurology 66.4, pp. 435-440. ISSN: 00039942. DOI: 10.1001/archneurol . 2009.15.

Pan, M. R. et al. (2019). "A comparison of efficacy between cognitive behavioral therapy (CBT) and CBT combined with medication in adults with attention-deficit/hyperactivity disorder (ADHD)". In: Psychiatry Research 279.June, pp. 23-33. ISSN: 18727123. DOI: $10.1016 / \mathrm{j}$. psychres.2019.06.040.

Park, B. Y., J. Kim, and H. Park (2016). “Differences in connectivity patterns between child and adolescent attention deficit hyperactivity disorder patients". In: Proceedings of the Annual International Conference of the IEEE Engineering in Medicine and Biology Society, EMBS. Vol. 2016-Octob, pp. 1127-1130. ISBN: 9781457702204. DOI: 10.1109/EMBC. 2016.7590902.

Pasternak, O. et al. (2012). "Excessive Extracellular Volume Reveals a Neurodegenerative Pattern in Schizophrenia Onset". In: Journal of Neuroscience 32.48, pp. 17365-17372. ISSN: 0270-6474. DOI: 10.1523/ JNEUROSCI . 2904-12. 2012.

Patz, S. et al. (2019). "Imaging localized neuronal activity at fast time scales through biomechanics". In: Science Advances 5.4. ISSN: 23752548. DOI: 10.1126/sciadv . aav3816.

Penny, W. D. et al. (2004). "Modelling functional integration: A comparison of structural equation and dynamic causal models". In: NeuroImage 23, S264-S274. ISSN: 10538119. DOI: 10 . 1016/ j . neuroimage. 2004.07.041.

Persson, J. et al. (2007). "Age differences in deactivation: A link to cognitive control?" In: Journal of Cognitive Neuroscience 19.6, pp. 1021-1032. ISSN: 0898929X. DOI: 10.1162/jocn. 2007 . 19.6.1021.

Pietschnig, J., M. Voracek, and A. K. Formann (2010). "Mozart effect-Shmozart effect: A metaanalysis". In: Intelligence 38.3. DOI: 10.1016/j . intell .2010.03.001. 
Plitt, M., K. A. Barnes, and A. Martin (2015). “Functional connectivity classification of autism identifies highly predictive brain features but falls short of biomarker standards". In: NeuroImage: Clinical 7, pp. 359-366. ISSN: 22131582. DOI: 10.1016/j.nicl.2014.12.013.

Power, J. D. et al. (2011). "Functional Network Organization of the Human Brain". In: Neuron 72.4, pp. 665-678. ISSN: 08966273. DOI: 10.1016/j. neuron.2011.09.006.

Preti, M. G., T. A. Bolton, and D. Van De Ville (2017). "The dynamic functional connectome: Stateof-the-art and perspectives". In: NeuroImage 160, pp. 41-54. ISSN: 10959572. DOI: $10.1016 / \mathrm{j}$. neuroimage.2016.12.061.

Pruessmann, K. P. et al. (1999). "SENSE: sensitivity encoding for fast MRI”. In: Magnetic Resonance in Medicine 42.5, pp. 952-962. DOI: 10 . 1002 / (SICI ) 1522 - 2594(199911) 42: 5<952 : : AID MRM16>3.0.CO;2-S.

Raichle, M. E. (2015). “The Brain's Default Mode Network”. In: Annual Review of Neuroscience 38, pp. 433-447. DOI: 10.1146/annurev-neuro-071013-014030.

Rane, P. et al. (2015). "Connectivity in Autism". In: Harvard Review of Psychiatry 23.4, pp. 223-244. ISSN: 1067-3229. DOI: $10.1097 / \mathrm{HRP} .0000000000000072$.

Rasero, J. et al. (2018). "Predicting functional networks from region connectivity profiles in taskbased versus resting-state fMRI data". In: PloS one 13.11, e0207385. ISSN: 19326203. DOI: 10 . 1371/journal . pone.0207385.

Rashid, B. et al. (2014). "Dynamic connectivity states estimated from resting fMRI Identify differences among Schizophrenia, bipolar disorder, and healthy control subjects". In: Frontiers in Human Neuroscience 8.November, pp. 1-13. ISSN: 1662-5161. DOI: 10.3389/fnhum. 2014. 00897.

Rashid, B. et al. (2016). "Classification of schizophrenia and bipolar patients using static and dynamic resting-state fMRI brain connectivity". In: NeuroImage 134, pp. 645-657. ISSN: 10959572. DOI: $10.1016 / j$.neuroimage.2016.04.051.

Rauscher, F. H., G. L. Shaw, and C. N. Ky (1993). "Music and spatial task performance”. In: Nature 365.611. DOI: $10.1038 / 365611 \mathrm{aO}$.

Redcay, E. (2008). "The superior temporal sulcus performs a common function for social and speech perception: Implications for the emergence of autism". In: Neuroscience $\mathcal{E}$ Biobehavioral Reviews 32.1, pp. 123-142. ISSN: 01497634. DOI: 10.1016/j . neubiorev . 2007.06.004.

Redcay, E. et al. (2013). "Intrinsic functional network organization in high-functioning adolescents with autism spectrum disorder". In: Frontiers in Human Neuroscience 7.September, pp. 1-11. ISSN: 1662-5161. DOI: 10.3389/fnhum. 2013.00573.

Regner, M. F. et al. (2016). “Top-Down Network Effective Connectivity in Abstinent Substance Dependent Individuals". In: PLoS One 11.10. DOI: 10.1371/journal . pone. 0164818.

Ren, P. et al. (2019). "Cognitive fatigue and cortical-striatal network in old age". In: Aging 11.8, pp. 2312-2326. ISSN: 19454589. DOI: 10.18632/aging. 101915.

Reuter-Lorenz, P. A. and K. A. Cappell (2008). "Neurocognitive aging and the compensation hypothesis". In: Current Directions in Psychological Science 17.3, pp. 177-182. ISSN: 09637214. DOI: 10.1111/j.1467-8721.2008.00570.x. arXiv: arXiv:1011.1669v3.

Roebroeck, A., E. Formisano, and R. Goebel (2005). "Mapping directed influence over the brain using Granger causality and fMRI". In: NeuroImage 25.1, pp. 230-242.

Roheger, M., E. Kalbe, and I. Liepelt-Scarfone (2018). "Progression of cognitive decline in Parkinson's disease". In: Journal of Parkinson's Disease 8.2, pp. 183-193. ISSN: 1877718X. DOI: 10.3233/ JPD- 181306.

Rolls, E. T. et al. (2018). "Effective Connectivity in Depression". In: Biological Psychiatry: Cognitive Neuroscience and Neuroimaging 3.2, pp. 187-197. ISSN: 24519030. DOI: 10.1016/j . bpsc . 2017.10 . 004.

Rosa, M. J. et al. (2015). "Sparse network-based models for patient classification using fMRI". In: Neuroimage 105, pp. 493-506. DOI: 10.1016/j . neuroimage.2014.11.021.

Rose, F. C. (2009). Chapter 39 An historical overview of British neurology. 3rd ed. Vol. 95. C. Elsevier B.V., pp. 613-628. ISBN: 9780444520098. DOI: 10.1016/S0072-9752(08) 02139-8. 
Sadeghi, M. et al. (2017). "Screening of autism based on task-free fMRI using graph theoretical approach". In: Psychiatry Research - Neuroimaging 263.February, pp. 48-56. ISSN: 18727506. DOI: 10.1016/j.pscychresns . 2017.02.004.

Sala-Llonch, R., D. Bartrés-Faz, and C. Junqué (2015). "Reorganization of brain networks in aging: a review of functional connectivity studies". In: Frontiers in Psychology 6.May, pp. 1-11. ISSN: 1664-1078. DOI: $10.3389 /$ fpsyg. 2015.00663.

Salehi, M. et al. (2020). "There is no single functional atlas even for a single individual: Functional parcel definitions change with task". In: NeuroImage 208, pp. 1-14. ISSN: 10538119. DOI: 10 .1016/ j. neuroimage. 2019.116366.

Sambataro, F. et al. (2017). "Altered dynamics of brain connectivity in major depressive disorder at-rest and during task performance". In: Psychiatry Research: Neuroimaging 259, pp. 1-9. ISSN: 09254927. DOI: 10.1016/j.pscychresns . 2016.11.001.

Schmaal, L. et al. (2016). "Subcortical brain alterations in major depressive disorder: Findings from the ENIGMA Major Depressive Disorder working group". In: Molecular Psychiatry 21.6, pp. 806812. ISSN: 14765578. DOI: $10.1038 / \mathrm{mp} .2015 .69$.

Schmaal, L. et al. (2017). “Cortical abnormalities in adults and adolescents with major depression based on brain scans from 20 cohorts worldwide in the ENIGMA Major Depressive Disorder Working Group". In: Molecular Psychiatry 22.6, pp. 900-909. ISSN: 14765578. DOI: 10 . 1038/mp . 2016.60.

Schneider-Garces, N. J. et al. (2010). "Span, CRUNCH, and Beyond: Working Memory Capacity and the Aging Brain". In: Journal of Cognitive Neuroscience 22.4, pp. 655-669. ISSN: 0898-929X. DOI: $10.1162 /$ jocn. 2009.21230. arXiv: NIHMS150003.

Schreiber, T. (2000). "Measuring information transfer". In: Physical Review Letters 85.2. DOI: 10 . 1103/PhysRevLett.85.461.

Sen, A., V. Capelli, and M. Husain (2018). "Cognition and dementia in older patients with epilepsy". In: Brain 141.6, pp. 1592-1608. ISSN: 14602156. DOI: 10.1093/brain/awy022.

Seth, A. K., A. B. Barrett, and L. Barnett (2015). "Granger Causality Analysis in Neuroscience and Neuroimaging". In: Journal of Neuroscience 35.8, pp. 3293-3297. ISSN: 0270-6474. DOI: $10.1523 /$ jneurosci.4399-14.2015.

Seth, A. K., P. Chorley, and C. Barnett (2012). "Granger causallity analysis of fMRI BOLD signals is invariant to hemodynamic convolution but not downsampling". In: Neuroimage 65, pp. 540-555. DOI: /10.1016/j . neuroimage. 2012.09.049.

Seth, A. K. (2010). "A MATLAB toolbox for Granger causal connectivity analysis". In: Journal of Neuroscience Methods 186.2, pp. 262-273. ISSN: 01650270. DOI: 10 .1016/ j . jneumeth . 2009 . 11. 020.

Seth, A. K., P. Chorley, and L. C. Barnett (2013). "Granger causality analysis of fMRI BOLD signals is invariant to hemodynamic convolution but not downsampling." In: NeuroImage 65, pp. 54055. ISSN: 1095-9572. DOI: 10.1016/j . neuroimage. 2012.09.049.

Shakil, S., C. H. Lee, and S. D. Keilholz (2016). "Evaluation of sliding window correlation performance for characterizing dynamic functional connectivity and brain states". In: NeuroImage 133, pp. 111-128. ISSN: 10959572. DOI: 10.1016/j . neuroimage. 2016.02.074.

Sharp, D. J. and P. O. Jenkins (2015). "Concussion is confusing us all". In: Practical Neurology 15.3, pp. 172-186. ISSN: 14747766. DOI: 10.1136/practneurol-2015-001087.

Shi, S., G. Bottegal, and P. M. J. Van den Hof (2019). "Bayesian topology identification of linear dynamic networks". In: 2019 18th European Control Conference (ECC), pp. 2814-2819. DOI: 10 . 23919/ECC. 2019.8795766.

Shirer, W. R. et al. (2012). "Decoding subject-driven cognitive states with whole-brain connectivity patterns". In: Cerebral Cortex 22.1, pp. 158-165. ISSN: 10473211. DOI: 10. 1093/cercor/bhr099.

Siman-Tov, T. et al. (2017). "Early age-related functional connectivity decline in high-order cognitive networks". In: Frontiers in Aging Neuroscience 8.January, pp. 1-17. ISSN: 16634365. DOI: 10.3389/fnagi.2016.00330. 
Smith, S. M. et al. (2012). "The danger of systematic bias in group-level FMRI-lag-based causality estimation". In: NeuroImage 59.2, pp. 1228-1229. ISSN: 10538119. DOI: $10.1016 / \mathrm{j}$. neuroimage. 2011.08 .015$.

Smith, S. M. and T. E. Nichols (2009). “Threshold-free cluster enhancement: Addressing problems of smoothing, threshold dependence and localisation in cluster inference". In: NeuroImage 44.1, pp. 83-98. ISSN: 10538119. DOI: 10.1016/j. neuroimage.2008.03.061.

Smith, S. M. et al. (2009a). "Correspondence of the brain's functional architecture during activation and rest." In: Proceedings of the National Academy of Sciences 106.31, pp. 13040-5. ISSN: 1091-6490. DOI: $10.1073 /$ pnas. 0905267106. arXiv: 0905267106.

- (2009b). "Correspondence of the brain's functional architecture during activation and rest." In: Proceedings of the National Academy of Sciences 106.31, pp. 13040-5. ISSN: 1091-6490. DOI: 10.1073/ pnas. 0905267106. arXiv: 0905267106.

Sofronoff, K., T. Attwood, and S. Hinton (2005). "A randomised controlled trial of a CBT intervention for anxiety in children with Asperger syndrome". In: Journal of Child Psychology and Psychiatry and Allied Disciplines 46.11, pp. 1152-1160. ISSN: 00219630. DOI: 10.1111 / j .14697610.2005.00411.x.

Song, J. et al. (2014). "Age-Related Reorganizational Changes in Modularity and Functional Connectivity of Human Brain Networks". In: Brain Connectivity 4.9, pp. 662-676. ISSN: 2158-0014. DOI: 10.1089 /brain. 2014.0286.

Spreng, R. N. et al. (2016). "Attenuated anticorrelation between the default and dorsal attention networks with aging: evidence from task and rest". In: Neurobiology of Aging 45, pp. 149-160. ISSN: 15581497. DOI: 10.1016/j.neurobiolaging. 2016.05.020.

Sprooten, E. et al. (2017). "Addressing reverse inference in psychiatric neuroimaging: Metaanalyses of task-related brain activation in common mental disorders". In: Human Brain Mapping 38.4, pp. 1846-1864. ISSN: 10970193. DOI: 10.1002/hbm. 23486.

Sridharan, D., D. J. Levitin, and V. Menon (2008). "A critical role for the right fronto-insular cortex in switching between central-executive and default-mode networks". In: Proceedings of the National Academy of Sciences 105.34, pp. 12569-12574. ISSN: 0027-8424. DOI: 10 . 1073 / pnas . 0800005105. arXiv: arXiv:1408.1149.

Stefan, H. et al. (2014). "Epilepsy in the elderly: Comparing clinical characteristics with younger patients". In: Acta Neurologica Scandinavica 129.5, pp. 283-293. ISSN: 16000404. DOI: 10 . 1111 / ane. 12218.

Suk, H. I. et al. (2016). "State-space model with deep learning for functional dynamics estimation in resting-state fMRI". In: NeuroImage 129, pp. 292-307. ISSN: 10959572. DOI: 10 . 1016/j . neuroimage.2016.01.005.

Taipale, H. et al. (2018). "Use of Antiepileptic Drugs and Dementia Risk—an Analysis of Finnish Health Register and German Health Insurance Data". In: Journal of the American Geriatrics Society 66.6, pp. 1123-1129. ISSN: 15325415. DOI: 10.1111/jgs.15358.

Teipel, S. J. et al. (2012). "Anatomical MRI and DTI in the diagnosis of Alzheimer's disease: A european multicenter study". In: Journal of Alzheimer's Disease 31.S3, pp. 33-47. ISSN: 18758908. DOI: $10.3233 /$ JAD-2012-112118.

Tomasi, D. and N. D. Volkow (2012). "Aging and functional brain networks". In: Molecular Psychiatry 17.5, pp. 549-558. ISSN: 13594184. DOI: 10.1038/mp. 2011.81.

Torrence, C. and G. P. Compo (1998). "A Practical Guide to Wavelet Analysis". In: Bulletin of the American Meteorological Society 79.1, pp. 61-78. ISSN: 0003-0007. DOI: 10.1175/1520-0477(1998) 079<0061: APGTWA>2 . 0. CO; 2.

Trinka, E. (2003). "Epilepsy: Comorbidity in the elderly". In: Acta Neurologica Scandinavica, Supplement 108.180, pp. 33-36. ISSN: 00651427. DOI: 10.1034/j.1600-0404.108.s180.5.x.

Tsvetanov, K. A. et al. (2016). “Extrinsic and Intrinsic Brain Network Connectivity Maintains Cognition across the Lifespan Despite Accelerated Decay of Regional Brain Activation". In: Journal of Neuroscience 36.11, pp. 3115-3126. ISSN: 0270-6474. DOI: 10 . 1523/ JNEUROSCI . 2733-15. 2016. 
Tyszka, J. M. et al. (2014). “Largely Typical Patterns of Resting-State Functional Connectivity in High-Functioning Adults with Autism". In: Cerebral Cortex 24.7, pp. 1894-1905. ISSN: 1047-3211. DOI: $10.1093 /$ cercor/bht040.

Tzourio-Mazoyer, N. et al. (2002). "Automated anatomical labeling of activations in SPM using a macroscopic anatomical parcellation of the MNI MRI single-subject brain". In: NeuroImage 15.1, pp. 273-289. ISSN: 10538119. DOI: 10.1006/nimg. 2001.0978.

Uddin, L. Q. et al. (2011). "Dynamic Reconfiguration of Structural and Functional Connectivity Across Core Neurocognitive Brain Networks with Development". In: Journal of Neuroscience 31.50, pp. 18578-18589. ISSN: 0270-6474. DOI: 10 . 1523 / JNEUROSCI . 4465 - 11 . 2011. arXiv: NIHMS150003.

Uddin, L. Q. and V. Menon (2009). "The anterior insula in autism: Under-connected and underexamined". In: Neuroscience and Biobehavioral Reviews 33.8, pp. 1198-1203. ISSN: 01497634. DOI: 10.1016/j.neubiorev. 2009.06.002.

Uddin, L. Q., K. Supekar, and V. Menon (2013). "Reconceptualizing functional brain connectivity in autism from a developmental perspective". In: Frontiers in Human Neuroscience 7.August, pp. 1-11. ISSN: 1662-5161. DOI: 10.3389/fnhum. 2013.00458.

Uddin, L. Q. et al. (2013). "Salience Network-Based Classification and Prediction of Symptom Severity in Children With Autism". In: JAMA Psychiatry 70.8, p. 869. ISSN: 2168-622X. DOI: 10 . 1001/jamapsychiatry. 2013.104. arXiv: NIHMS150003.

Uddin, L. Q. et al. (2015). "Brain state differentiation and behavioral inflexibility in autism". In: Cerebral Cortex 25.12, pp. 4740-4747. ISSN: 14602199. DOI: 10.1093/cercor/bhu161.

Vanhaudenhuyse, A. et al. (2010). “Default network connectivity reflects the level of consciousness in non-communicative brain-damaged patients". In: Brain 133.1, pp. 161-171. ISSN: 14602156. DOI: $10.1093 /$ brain/awp313.

Veer, I. M. et al. (2010). "Whole brain resting-state analysis reveals decreased functional connectivity in major depression." In: Frontiers in systems neuroscience 4.September, pp. 1-10. ISSN: 16625137. DOI: $10.3389 /$ fnsys. 2010.00041.

Verrusio, W. et al. (2015). "The Mozart effect: a quantitative EEG study". In: Consciousness and Cognition 35, pp. 150-155. DOI: 10.1016/j. concog.2015.05.005.

Vossel, S., J. J. Geng, and G. R. Fink (2014). “Dorsal and ventral attention systems: Distinct neural circuits but collaborative roles". In: Neuroscientist 20.2, pp. 150-159. ISSN: 10894098. DOI: 10 . $1177 / 1073858413494269$.

Wang, D. et al. (2015). "Parcellating cortical functional networks in individuals". In: Nature Neuroscience 18.12, pp. 1853-1860. ISSN: 15461726. DOI: 10.1038/nn. 4164.

Wang, J. (2010). "Graph-based network analysis of resting-state functional MRI". In: Frontiers in Systems Neuroscience 4.June, pp. 1-14. ISSN: 16625137. DOI: 10.3389/fnsys . 2010.00016.

Wang, N., W. Zeng, and L. Chen (2012). "A Fast-FENICA method on resting state fMRI data". In: Journal of Neuroscience Methods 209.1, pp. 1-12. ISSN: 01650270. DOI: 10.1016/j . jneumeth. 2012. 05.007.

Wang, X. et al. (2011). "Abnormalities in Resting-State Functional Connectivity in Early Human Immunodeficiency Virus Infection". In: Brain Connectivity 1.3, pp. 207-217. ISSN: 2158-0014. DOI: 10.1089/brain.2011.0016.

Wang, Y., M. Sznaier, and O. Camps (2016). "A Super-Atomic Norm Minimization Approach to Identifying Sparse Dynamical Graphical Models". In: American Control Conference (ACC). IEEE, pp. 1962-1967. DOI: 10.1109/ACC. 2016.7525206.

Wardlaw, J. M., M. C. Valdés Hernández, and S. Muñoz-Maniega (2015). “What are white matter hyperintensities made of? Relevance to vascular cognitive impairment". In: Journal of the American Heart Association 4.6, e001140. ISSN: 20479980. DOI: 10.1161/JAHA .114.001140.

Watanabe, T. and G. Rees (2017). "Brain network dynamics in high-functioning individuals with autism". In: Nature Communications 8.May, pp. 1-14. ISSN: 20411723. DOI: 10. 1038/ncomms 16048. 
Wechsler, D. (2008). “WAIS-IV : Wechsler adult intelligence scale". In: San Antonio, TX: Psychological Corporation. ISBN: 9780158980843. DOI: 10.1016/S0043-1354(02)00019-2.

Wei, M. et al. (2013). "Identifying major depressive disorder using Hurst exponent of resting-state brain networks". In: Psychiatry Research - Neuroimaging 214.3, pp. 306-312. ISSN: 09254927. DOI: 10.1016/j.pscychresns. 2013.09.008.

Weitz, J. S. et al. (2016). "Replicator Dynamics with Feedback-Evolving Games: Towards a CoEvolutionary Game Theory". In: bioRxiv, pp. 1-10. DOI: 10.1101/043299.

Wen, X., G. Rangarajan, and M. Ding (2013). "Is Granger Causality a Viable Technique for Analyzing fMRI Data?" In: PLoS ONE 8.7, e67428. ISSN: 19326203. DOI: 10.1371 / journal . pone . 0067428.

Whitehead, J. C. and J. L. Armony. "Singing in the brain: Neural representation of music and voice as revealed by fMRI". In: Human Brain Mapping 39.12, pp. 4913-4924.

Wicker, B. et al. (2008). "Abnormal cerebral effective connectivity during explicit emotional processing in adults with autism spectrum disorder". In: Social Cognitive and Affective Neuroscience 3.2, pp. 135-143. ISSN: 17495016. DOI: $10.1093 / \mathrm{scan} / \mathrm{nsn} 007$.

Wig, G. S. (2017). “Segregated Systems of Human Brain Networks". In: Trends in Cognitive Sciences 21.12, pp. 981-996. ISSN: 1879307X. DOI: 10.1016/j.tics.2017.09.006.

Winkler, A. M. et al. (2014). "Permutation inference for the general linear model". In: NeuroImage 92, pp. 381-397. ISSN: 10959572. DOI: 10.1016/j .neuroimage. 2014.01.060.

Xiong, T. and V. Cherkassky (2005). "A combined SVM and LDA approach for classification". In: Proceedings of the International Joint Conference on Neural Networks 3, pp. 1455-1459. DOI: 10.1109/ I JCNN. 2005.1556089.

$\mathrm{Xu}, \mathrm{H}$. et al. (2018). "Impact of global signal regression on characterizing dynamic functional connectivity and brain states". In: NeuroImage 173.January, pp. 127-145. ISSN: 10959572. DOI: $10.1016 / j$.neuroimage. 2018.02.036.

Yaesoubi, M. et al. (2015). "Dynamic coherence analysis of resting fMRI data to jointly capture state-based phase, frequency, and time-domain information." In: NeuroImage 120, pp. 133-42. ISSN: 1095-9572. DOI: 10.1016/j . neuroimage.2015.07.002. arXiv: arXiv: 1505.06832v1.

Yamada, T. et al. (2017). "Resting-state functional connectivity-based biomarkers and functional mri-based neurofeedback for psychiatric disorders: A challenge for developing theranostic biomarkers". In: International Journal of Neuropsychopharmacology 20.10, pp. 769-781. ISSN: 14695111. DOI: 10.1093/ijnp/pyx059.

Yang, M. et al. (2018). "The Effects of Music Intervention on Functional Connectivity Strength of the Brain in Schizophrenia". In: Neural Plasticity 2018, 2821832. DOI: 10.1155/2018/2821832.

Yeo, B. T. T. et al. (2011). "The organization of the human cerebral cortex estimated by intrinsic functional connectivity". In: Journal of Neurophysiology 106.3, pp. 1125-1165. ISSN: 0022-3077. DOI: $10.1152 /$ jn. 00338.2011.

Yerys, B. E. et al. (2015). “Default mode network segregation and social deficits in autism spectrum disorder: Evidence from non-medicated children DMN in children with ASD". In: NeuroImage: Clinical 9, pp. 223-232. ISSN: 22131582. DOI: 10.1016/j.nicl.2015.07.018.

Zang, Y. F. et al. (2007). "Altered baseline brain activity in children with ADHD revealed by resting-state functional MRI". In: Brain and Development 29.2, pp. 83-91. ISSN: 03877604. DOI: 10.1016/j. braindev. 2006.07.002.

Zaremba, A. and T. Aste (2014). Measures of Causality in Complex Datasets with Application to Financial Data. Vol. 16. 4, pp. 2309-2349. ISBN: 4402073871. DOI: 10 .3390/e16042309. arXiv: arXiv: $1401.1457 \mathrm{v} 1$.

Zhenyu Liu et al. (2012). "Exploring the effective connectivity of resting state networks in Mild Cognitive Impairment: An fMRI study combining ICA and multivariate Granger causality analysis". In: 34th Annual International Conference of the IEEE Engineering in Medicine and Biology Society. Vol. 2012. IEEE, pp. 5454-5457. ISBN: 978-1-4577-1787-1. DOI: 10.1109/EMBC. 2012 .6347228. 
Zhou, J. et al. (2017). “Applications of Resting-State Functional Connectivity to Neurodegenerative Disease". In: Neuroimaging Clinics of North America 27.4, pp. 663-683. ISSN: 15579867. DOI: 10 . 1016/j.nic.2017.06.007.

Zhou, Y. et al. (2010). "Spontaneous brain activity observed with functional magnetic resonance imaging as a potential biomarker in neuropsychiatric disorders". In: Cognitive Neurodynamics 4.4, pp. 275-294. ISSN: 18714080. DOI: $10.1007 / \mathrm{s} 11571-010-9126-9$.

Zhou, Z. et al. (2011). "A conditional Granger causality model approach for group analysis in functional magnetic resonance imaging". In: Magnetic Resonance Imaging 29.3, pp. 418-433. ISSN: 0730725X. DOI: 10.1016/j.mri.2010.10.008.

Zingone, R., C. Mocenni, and D. Madeo (2018). “Evolutionary Games, Complex Networks and Nonlinear Analysis for Epileptic Seizures Forecasting". In: arXiv: 1812.03078.

Zuo, X. N. et al. (2010). "Reliable intrinsic connectivity networks: Test-retest evaluation using ICA and dual regression approach". In: NeuroImage 49.3, pp. 2163-2177. ISSN: 10538119. DOI: 10 . 1016/j . neuroimage. 2009.10.080.

Zweiphenning, W. J.E. M. et al. (2019). “Increased gamma and decreased fast ripple connections of epileptic tissue: A high-frequency directed network approach". In: Epilepsia 60.9, pp. 1908-1920. ISSN: 0013-9580. DOI: 10.1111/epi.16296. 



\section{List of publications}

\section{Journal publications}

Bernas, A., Breuer, L. E. M., Lamerichs, R., de Louw, A. J. A., Aldenkamp, A. P., Zinger, S. (2020). Accelerated Cognitive Ageing in epilepsy: exploring the effective connectivity between resting-state networks and its relation to cognitive decline. Heliyon, 6(6), e03951. DOI: 10.1016/j.heliyon.2020.e03951.

Breuer, L. E. M., Carrette, E. C. B., Boon, P., Bernas, A., Besseling, R. M. H., Aldenkamp, A. P., de Louw, A. (2019). Accelerated Cognitive Ageing in Epilepsy: A Neuropsychological Evaluation of Cognitive Deterioration. Archives of Clinical Neuropsychology, 34(3), 301-309. DOI: 10.1093/arclin/acy042.

Bernas, A., Aldenkamp, A. P., Zinger, S. (2018). Wavelet coherence-based classifier: A resting-state functional MRI study on neurodynamics in adolescents with high-functioning autism. Computer Methods and Programs in Biomedicine, 154, 143-151.

DOI: $10.1016 /$ j.cmpb.2017.11.017.

Bernas, A., Barendse, E. M., Aldenkamp, A. P., Backes, W. H., Hofman, P. A. M., Hendriks, M. P. H., Kessels, R. P. C., Willems, F. M. J., de With, P. H. N., Zinger, S., Jansen, J. F. A. (2018). Brain resting-state networks in adolescents with high-functioning autism: Analysis of spatial connectivity and temporal neurodynamics. Brain and Behavior, 8(2), 1-10. DOI: 10.1002/brb3.878.

Breuer, L. E. M., Grevers, E., Boon, P., Bernas, A., Bergmans, J. W. M., Besseling, R. M. H., Klooster, D. C. W., de Louw, A., Mestrom, R. M. C., Vonck, K., Zinger, S., Aldenkamp, A. P. (2016). Cognitive deterioration in adult epilepsy: clinical characteristics of "Accelerated Cognitive Ageing." Acta Neurologica Scandinavica, September, 1-7. DOI: 10.1111/ane.12700.

Bernas, A. (2016). Neurodynamics in functional MRI and its clinical applications. Eindhoven: Technische Universiteit Eindhoven. ISBN: 978-90-444-1444-8

Breuer, L. E. M., Boon, P., Bergmans, J. W. M., Mess, W. H., Besseling, R. M. H., de Louw, A., Tijhuis, A. G., Zinger, S., Bernas, A., Klooster, D. C. W., Aldenkamp, A. P. (2016). Cognitive deterioration in adult epilepsy: Does accelerated cognitive ageing exist? Neuroscience Biobehavioral Reviews, 64,1-11. DOI: 10.1016/j.neubiorev.2016.02.004.

Klooster, D. C. W., de Louw, A. J. A., Aldenkamp, A. P., Besseling, R. M. H., Mestrom, R. M. C., Carrette, S., Zinger, S., Bergmans, J. W. M., Mess, W. H., Vonck, K., Carrette, E., Breuer, L. E. M., Bernas, A., Tijhuis, A. G., Boon, P. (2016). Technical aspects of neurostimulation: Focus on equipment, electric field modeling, and stimulation protocols. Neuroscience and Biobehavioral Reviews, 65, 113-141. DOI: 10.1016/j.neubiorev.2016.02.016. 


\section{Conference contributions}

Bernas, A., Zinger, S., Aldenkamp, A. (2016). Wavelet coherence-based classifier: fMRI study for high-functioning autism. In ESMRMB 2016, 33rd Annual Scientific Meeting, Vienna, AT, September 29 - October 1: Abstracts, Saturday: Book of Abstracts (pp. S337). [358] (Magnetic Resonance Materials in Physics, Biology and Medicine; Vol. 29, No. Supplement 1). Springer.

Bernas, A., Barendse, E. M., Zinger, S., Aldenkamp, A. P. (2016). Wavelet-based coherence between large-scale resting-state networks: neurodynamics marker for autism? In Proceedings of the 37th WIC Symposium on Information Theory in the Benelux, and The 6th Joint WIC/IEEE Symposium on Information Theory and Signal Processing in the Benelux., Louvain-la-Neuve, Belgium, May 19-20, 2016 (pp. 58-65). Louvain-la-Neuve: Katholieke Universiteit Leuven. 


\section{Valorisation}

\section{Main findings}

In this thesis, new ways of modelling brain dynamics using functional magnetic resonance imaging (fMRI) are described. A measure of causality, synchronicity, and emulative power between resting-state brain networks were assessed in two clinical populations: autism adolescents and elderly with accelerating cognitive ageing (ACA). The main findings are: (i) time of in-phase coherence extracted from wavelet coherence approach, i.e. the measure of synchronicity between brain networks, is a good descriptor of the autistic disorder, and allow the fMRI-based diagnosis of autism with high accuracy (> 85\%); (ii) the evolutionary game theory approach and its derived emulative power between brain networks combined with wavelet coherence, Granger causality, and static functional connectivity approaches could classify ACA from non-ACA with more than 95\% accuracy; (iii) Granger causality gives relevant information about the direction of the brain network connectivity involved in the deficiency displayed in autism or ACA, but is not a strong biomarker (classifiers fell short of providing high accuracy performances), whereas a new Bayesian topology modelling for describing causality in the brain dynamics seems to have added value (compared to Granger causality) and should be explored further.

\section{Relevance}

Mental disorders are estimated to be prevalent in $18 \%$ of Dutch population (18 to 64 years old), with the most commons psychological disorders being: depression, specific phobia, social phobia, alcohol abuse and ADHD, according to the Dutch organisation of mental health $\left(\mathrm{GGZ}^{1}\right)$. And even though mental health problems are the major cause of burden of disease, mental health providers only use $6.1 \%$ of total health and social care budget, in the Netherlands. Unfortunately, in addition, treatments for neuropsychiatric patients are known to be often ineffective due to misdiagnosis or the poorly understood aetiology of the conditions. Two solutions can be proposed for tackling these issues: allocating more healthcare and social care budgets for research in psychiatry, or distributing these resources in a more optimal way, i.e. with advances in techniques that ensure reliable diagnoses and treatment effect prognostics.

Knowing more about the mechanisms of action of a mental disorder can greatly help with avoiding misdiagnosis and designing targeted and more effective treatment solutions. The findings of the research presented in this thesis are actually relevant for psychoradiology, the field of research studying psychology through the lens of brain images; and hence, relevant for advancing the study of brain pathologies, and more specifically, the (neuro)psychiatric disorders. Therefore, if this research is pushed forward with better understanding of the pathological brain dynamics, treatment that would target and affect these dynamics can be developed; and misdiagnoses may be also avoided.

\footnotetext{
${ }^{1}$ https://www.denederlandseggz.nl/
} 
In addition, this research allows the follow-up of the progression of neuropsychiatric disorders by helping in screening the brain dynamics changes and/or through visualisation and analysis of treatment effects, using fMRI scans.

Overall, advances in psychoradiology, as presented in this thesis, can be relevant and beneficial for the healthcare system with regard to mental health and its cost-efficient budget allocation.

\section{Target groups}

This research is not only relevant for the field of psychoradiology and its societal effect on the healthcare system. Indeed, this thesis is directly targeting patients with autism and elderly with cognitive decline problems. And more generally, the research also aims at improving the lives of patients with neurological or psychological disorders, since these new methods for assessing brain dynamics could be used for understanding mechanisms of action of other mental disorders.

In addition to the patients, clinicians can profit from this type of research and development by having new tools to assess brain disorders; and brain dynamics visualisation and understanding can facilitate the diagnosis or the prediction of treatment response in case of neurological or psychiatric conditions. This research has, overall, the potential to re-assure objectively clinicians' decisions regarding the diagnosis of a disorder or the prescription of therapies.

Furthermore, another target group that would benefit from this research may be new service developers. Startups such as Icometrix ${ }^{2}$ or Advantis $^{3}$ can very much be interested in new features allowing the visualisation of cognitive processes and descriptive markers of disorders, when structural imaging and other anatomical markers are not sufficient.

Finally, not only startups in post-processing image-based diagnostics but also MRI scanner vendors (e.g. Philips, or Siemens) could use the results presented in this thesis for new products. Indeed, this research and the impact it can have on the healthcare system and brain-diseased patients may incentivise vendors to develop more precise and specific hardware together with advanced MRI sequences for extracting robust neuropsychological brain markers.

\section{Activities and products}

As mentioned in the previous paragraph, new companies are being developed to offer services helping clinicians in the decision-making process for diagnosing brain conditions; and vendors of MRI scanners could incorporate the methods for accessing brain dynamics described in this thesis in their clinical and research software. These new tools would allow radiologists and clinicians to have a quicker and insightful look into the brains of the scanned patients. These critical insights put in clinical practice would alleviate the issue of misdiagnosis, and can also reassure the clinicians in their decisions (regarding diagnosis and treatment). So, new products can be developed from this neuroimaging research: e.g., brain dynamics visualisation software or reliable biomarkers extraction tools. Combining these products could lead to a strong computer-aided automated diagnostic tool.

\footnotetext{
${ }^{2}$ https://icometrix.com/

${ }^{3}$ https://advantis.io/
} 
One activity we could think of is to integrate these new brain dynamics extraction methods into existing brain visualisation and analytic software (e.g. BrainVoyager ${ }^{4}$, or the FSL software package $^{5}$. Another activity could be to patent the process of diagnosis/prognosis of a disease: from the scanning protocol and image processing pipeline to the automated classification and description of a patient's condition. Similar ideas of image-based technology for prediction of diseases has already got granted patents (e.g. Siemens' patent US7949167B2).

This research can also be extended to the study of more localized and deeper neuronal dynamics. Indeed, the findings of this thesis could be utilised for reading brain signals extracted from more advanced imaging techniques, such as brain-chip neural recording. For example, Neuralink ${ }^{6}$ is making great advances in neuronal recording and stimulation, which can provide a clearer (than the currently-used fMRI and EEG techniques) picture of activity in the brain. This research could greatly help to decipher and disentangle the dynamics of the large neuronal time-series data at a finer scale (than large-scale network dynamics observe in behaviour and cognition). This can help to design new solutions to restore functions in neurologically impaired patients (e.g. restoring sight, hearing, or motor control).

\section{Innovation, future work and implementation}

This thesis contains innovation for analysing brain dynamics. So far, brain fMRI images were assessed through correlation, giving clinicians and researchers static maps and metrics of brain network topology. Now, with novel insights into the dynamic processes of the brain, we saw that the brain functional topology can evolve. And most pathologies reside and can be described in this dynamic topology. In addition, this view of assessing the communication between largescale networks, rather than within all possible connections, is novel. It simplifies substantially the complexity of the models, but also allows understandable conclusions regarding abnormal brain connectivity - it is easier to describe high-level communication mechanisms to understand behavioural issues rather than modelling complex neuronal connectivity that hardly explains the emergence of pathological cognition and behaviours.

More innovation could be made in the data acquisition, with multi-modal techniques for instance. Coupling the high temporal resolution of an EEG and the high spatial resolution of MRI scans could extend the range of products and commercial activities for modern psychoradiological tools. Mixing structural data from diffusion-weighted imaging with fMRI-based brain features could also improve models for neuropsychiatric classification.

The development of these new tools, products and activities for neuropsychiatric diagnosis, or visualisation software for treatment strategies could be clinically implemented. However, before having these diagnostic and visualisation tools implemented in clinical practice, the research in psychoradiology for neuropsychiatric disorders must be consolidated. Fortunately, more and more research institutes provide the world with open access, and freely available anonymised datasets (e.g. OpenNeuro ${ }^{7}$ and $\mathrm{HCP}^{8}$ repositories (. However, these medical imaging data centres are usually constituted of anatomical brain images; but once similar data centres provide brain functional MRI data of neuropsychiatric patients and controls, more research will be conducted to improve and refine the models presented in this thesis. This will ultimately lead the neuropsychiatric field towards personalised (and disease-specific) diagnostic and treatment solutions.

\footnotetext{
${ }^{4}$ http://www. brainvoyager.com/

${ }^{5}$ https://fsl.fmrib.ox.ac.uk/fsl/fslwiki/

${ }^{6}$ https://neuralink.com/

${ }^{7}$ https://openneuro.org/

${ }^{8}$ http: //www.humanconnectomeproject.org/
} 
We could expect machine learning applications for the detection of robust biomarkers of neuropsychiatric to be developed in the coming decade. This time-frame is not unrealistic, since machine learning and AI algorithms are being developed currently in the field of medicine. Plus, this research in neurodynamics using fMRI can be easily performed in parallel with neuropsychological clinical evaluation practices. Indeed, the idea for further development is to simply add one or two MRI scanning sessions to the already performed surveys and psychological evaluations. And this add-up in the evaluation of neuropsychiatric disorders does not enlarge the burden of the patients and clinicians; whereas it actually enlarges the data collections in the data centres aforementioned, and hence, the research possibilities. Moreover, all the analytic research and data collection can be done at relatively no cost-if radiological costs are traded off with better targeted treatments - which is of benefit for the healthcare system and the society. Finally, machine learning data service startups, and scanner vendor companies could see, in these neuroimaging technologies, a great opportunity to developed further and for a broader costumer audience their commercial activities in healthcare and mental well-being. 


\section{Acknowledgements}

I would like to thank first and foremost Prof. dr. Bert Aldenkamp who has given me the opportunity to work in the exciting field of neuroscience, and believed in me all these years since my master's project. You have been a great mentor and promotor, and an inspiration for conducting this research. I appreciate the helpful inputs you gave me about the clinical meaning and implications of my research results. I also thank you for the valuable guidance and advice on how to be in charge of a research project and how to develop skills necessary in the professional environment.

I am very grateful to my co-promotor and supervisor Dr. Sveta Zinger. It has been a pleasure to have all these numerous meetings and conversations with you. Thanks for helping me in organising my research, and thought process. I appreciate the effort you have put in supervising me regardless of your busy schedules and other responsibilities. Many thanks for the personal support, I always thought I could count on you at any moments and not only for professional matters. I also loved the independency and autonomy you had given me to conduct my research while providing guidance and subtle but fruitful advice on how to proceed further in my work, but also in life.

I am also thankful to the Assessment Committee members, Prof. dr. R. van Oostenbrugge, Prof. dr. M. Breeuwer, Prof. dr. R. van Ee, and Prof. dr. D. Linden who reviewed this thesis and allowed me to defend it. In that regard, I also thank Maastricht University for funding most parts of my research and the administration staff for their help with all the necessary arrangements for the PhD ceremony.

I would like to thank Eindhoven University of Technology (TU/e) as well, and more specifically the VCA lab, from the Signal Processing System group of Electrical Engineering department. Thanks to Prof. dr. ir. Peter de With (head of the group) for funding parts of my research and allowing me to have an office at TU/e. I am thankful for having met great colleagues from this group; and some of them have become good friends now.

Warm thanks to the Kempenhaeghe medical centre that also funded parts of my research. the clinical and technical staff who runs the MRI facilities has been more than helpful for collecting the brain imaging data so important and critical to my project and this thesis. Special thanks to Remco and Rolf for helping me to set up the scan protocol.

Thanks to all the colleagues with whom I had the pleasure to work with. In particular, I am grateful to my clinical "twin" Dr. Lisanne Breuer, for conducting all the psychological analysis in the accelerated cognitive ageing patients and helping me to find biomarkers of this neuropsychiatric disorder. Your work has been very valuable for this dissertation. I also thank my office mates and NeuroPlatform colleagues: Sveta, Rene, Stephan, Rolf, Debby, Gerhard and Jesper for bringing some life in the office. I am glad we had those interesting interactions and even debates on some topics related or not to brain science. I also thank you for helping me preparing slides and presentations for teaching, conference talks, or poster presentations. I also thank the master's students I had the pleasure to supervise and collaborate with. Your inputs and contributions to my research has been immensely useful. 
Finally, I am thankful to my friends and family. Thanks to my old French friends "copains calin": Alex, Cam, Max, Elea, Fop, Sof, Fab, Clem, Tom, Anaïs, Cycy, Ludivine, Bapt, Fanny, Jojo and Claire I keep meeting every summer. You are awesome, supported me all those years and I am really glad to have you guys as close friends. I want to show my gratitude to the more recent friendships I have made in Eindhoven as well. In particular, thanks to the "Greek Lunch": Roger, Panos, Arash, Eleni, Chara, Francesca, Cristina, and Nailia. I am grateful to have this gang around me for everyday lunch, and for having those nice parties and holiday trips together. I want to thank Music with Stranger (MwS) association too. I have met great musicians over the years I have lived in Eindhoven thanks to MwS. Thank you guys for all those social gatherings, rehearsals and live performances. Many thanks to my parents, brothers and sisters who have supported me throughout my whole life and have always encouraged my development, believing in me with full understanding and respect of my decisions. I want to thank, at last, Nailia Nasibulina, not only for the considerable help in reviewing the introduction and the conclusion of this thesis, but certainly for the emotional support and for being a great partner in life and love.

Many thanks to all of you! 


\section{Curriculum Vitae}

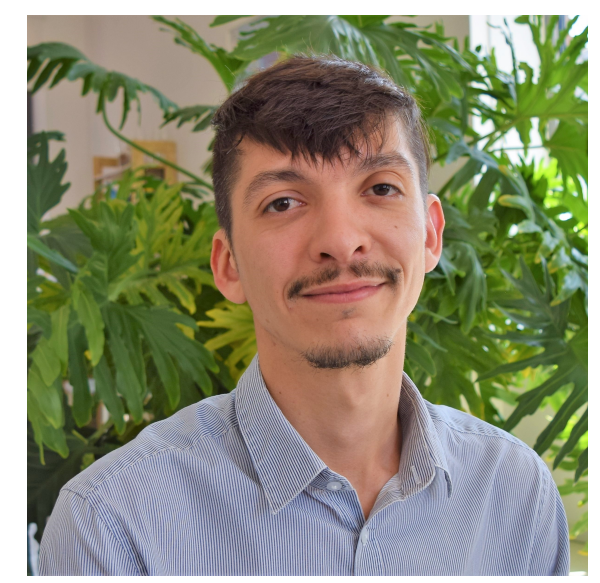

Antoine Bernas was born on May 21990 in Avignon, France. After growing up in the region of Brittany, he obtained a master's degree in computer science and electrical engineering in Brest, France. Antoine's master's final project was done at Eindhoven University of Technology (TU/e), the Netherlands.

After the graduation of his Master in 2013, Antoine stayed in Eindhoven and enrolled in the two-year PDEng program in Healthcare System Design in the Electrical Engineering department of TU/e. During that period, he worked on developing new tools to assess brain dynamics using resting-state fMRI.

After obtaining his PDEng diploma in February 2016, Antoine continues working in the field of MRI and neurodynamics as a PhD candidate within the NeuroPlatform program at TU/e. This program, led by Prof. dr. A.P. Aldenkamp, is a collaboration between Maastricht University, Kempenhaeghe Hospital and TU/e. Together with his supervising team (Prof. dr. A.P. Aldenkamp and Dr. S. Zinger), he has focused on neurodynamics analysis of resting-state fMRI data of elderly epilepsy patients with cognitive decline. The various results of this research have led to several publications in scientific journals, which are aggregated and presented in this thesis.

Since 2016, Antoine have also had the pleasure to give lectures and supervise master's and bachelor's students in medical imaging, MRI analysis, and neuro-engineering topics.

Beside his passion for scientific research in neuroscience and neuroimaging, Antoine likes to play the drums and perform on stage, enjoys racket sports, and never refuses a chess game. 